Aus der Abteilung Humangenetik

(Prof. Dr. med. Dr. h.c. W. Engel)

im Zentrum Hygiene und Humangenetik

der Medizinischen Fakultät der Universität Göttingen

\title{
MicroRNA Expression Profiling of Multipotent Adult Germline Stem Cells
}

\author{
Inaugural-Dissertation \\ zur Erlangung des Doktorgrades \\ der Medizinischen Fakultät \\ der Georg-August-Universität zu Göttingen
}

vorgelegt von

Athanasios Zovoilis

aus Amarousio Attikis, Griechenland

Göttingen 2008 
Dekan:

I.Berichterstatter:

II.Berichterstatter/in:

III.Berichterstatter/in:

Tag der mündlichen Prüfung:
Prof. Dr. med. C. Frömmel

Prof. Dr. med. Dr. h.c. W. Engel 
Contents....................................................................................

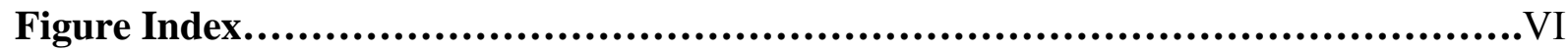

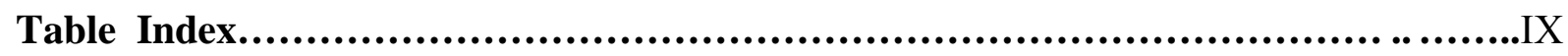

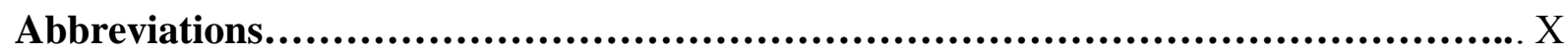

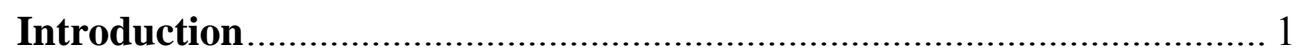

$1.1 \quad$ Definitions and general concepts about pluripotent cells ............................ 1

1.1.1 Stem cells and their differentiation potential during development............... 1

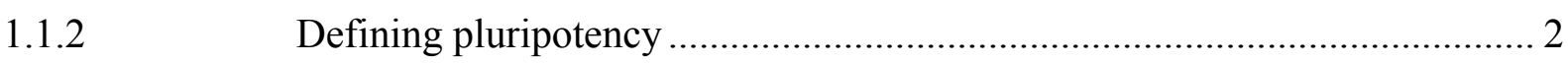

Types of pluripotent cells in mouse ..................................................... 3

1.2 Multipotent adult germline stem cells in mouse and their potential............. 5

Critical pathways controlling pluripotency in mouse ….............................. 7

microRNAs in pluripotency and development ..................................... 8

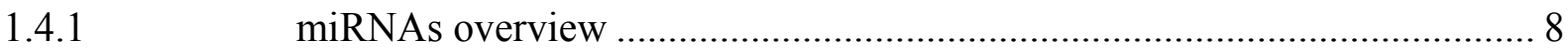

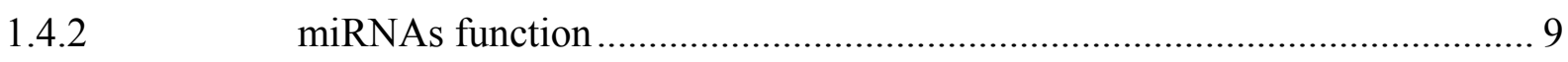

1.4.3 Embryonic stem cell specific miRNAs (ESC-specific miRNAs) ................ 11

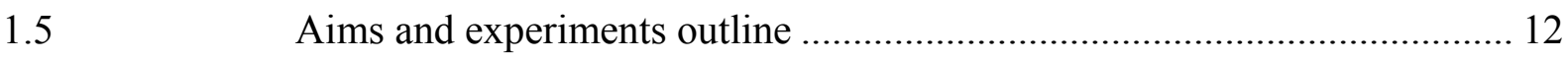

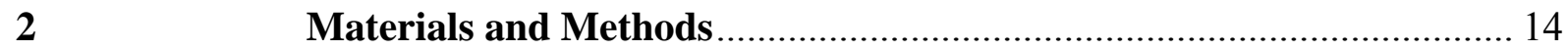

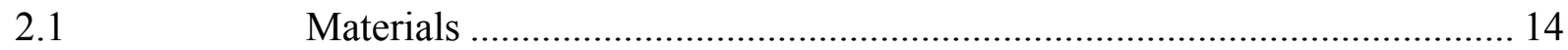

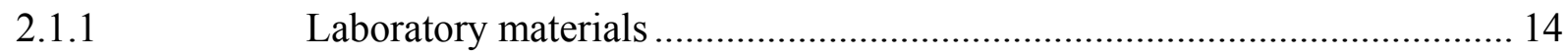

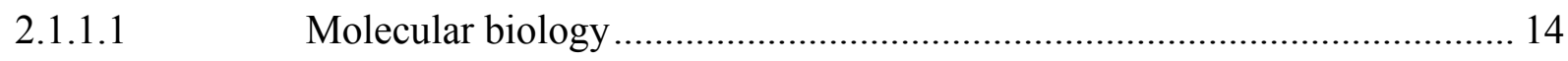

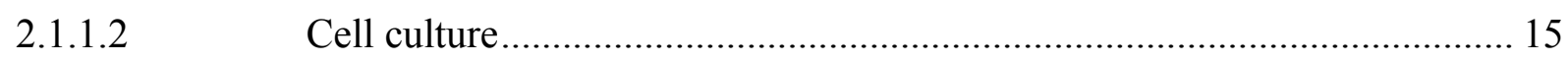

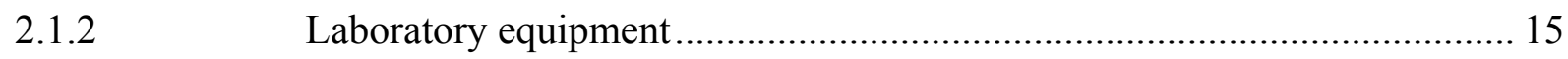

2.1.3 Chemicals, biochemical and biological materials..................................... 16

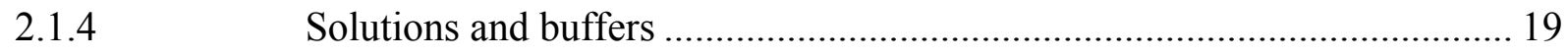

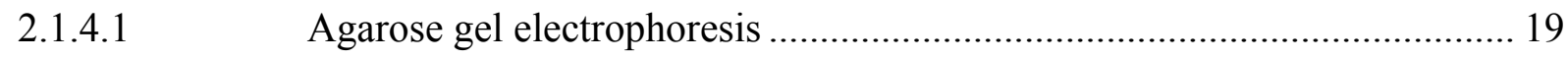

2.1.4.2 Frequently used buffers and solutions ................................................ 19

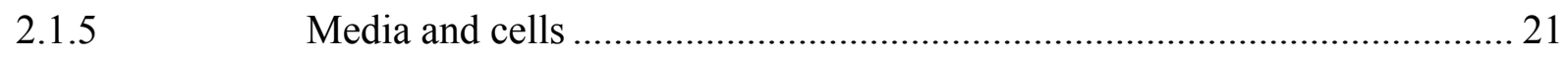

2.1.5.1 Eukaryotic cell culture media and cells ................................................. 21

2.1.5.2 Bacteria culture media and cells .............................................................. 22 
Preparation, maintenance and cryopreservation of MEFs and feeder layers 27

Culture of ESCs and maGSCs 28

Culture of F9 cells. 29

RNA and microRNA expression analysis (Reverse Transcription PCR) .... 35

Real-time RT-PCR (qPCR) for mRNAs .............................................. 41 


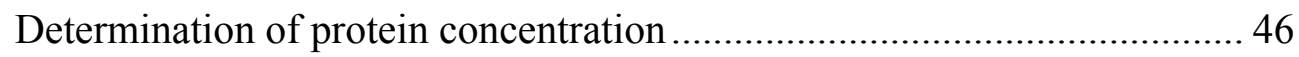

Expression of miR-290 and miR-302 miRNA clusters in ESCs and maGSCs after culture for many passages and upon loss of pluripotency.... 62 Expression profiles of miR-290 and miR-302 miRNA clusters during in vitro differentiation of pluripotent cells 66

Expression profile of miR-290 cluster during in vitro differentiation of ESCs and maGSCs.

3.5.4 Expression profile of miR-302 cluster during in vitro differentiation of ESCs and maGSCs. 
Determination of the differentiation state of the cells tested

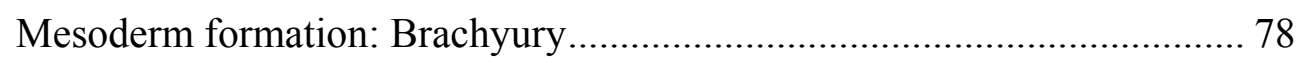

Endoderm formation: Hnf-4 78

Correlation of the differentiation state of the cells with miRNA levels of miR-290 and miR-302 clusters.

Correlation of miR-290 with Oct-4 expression (based on differences between different conditions) .....

Correlation of miR-290 with Oct-4 expression and differentiation (based on differences between different cell types).

Determination of de novo DNA methyltransferases expression profile in ESCs and maGSCs.

The metazoan microRNAomes and the importance of miRNA-mediated regulation of gene expression .....

Members of miR-290 family are connected with maintenance of pluripotency

4.4.2 Members of miR-302 family are induced during first stages of in vitro differentiation..

4.4.3 Further proofs about connection of these miRNAs with pluripotency or differentiation 


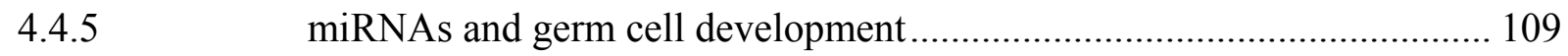

4.5 miRNAs and de novo DNA methylation in maGSCs ............................... 111

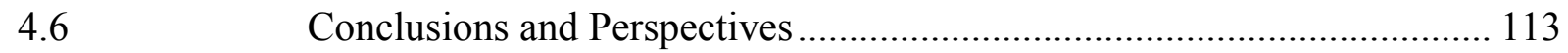

5

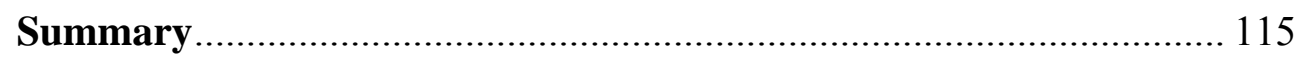

6

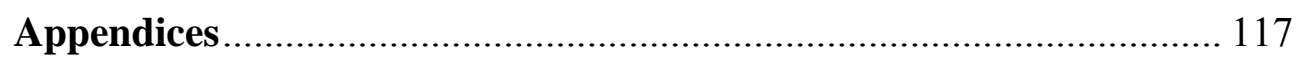

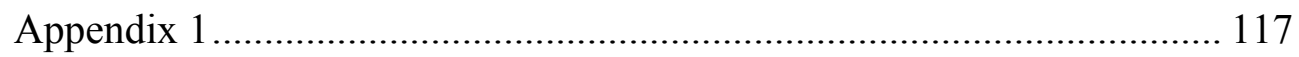

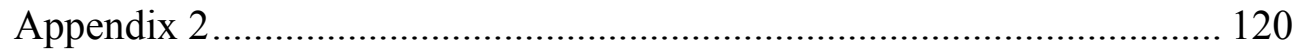

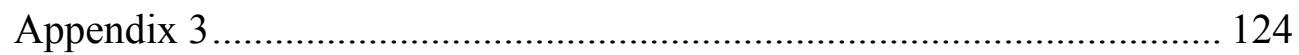

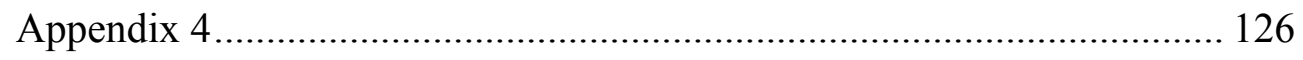

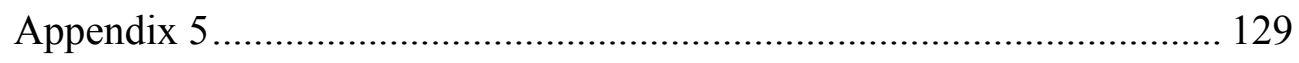

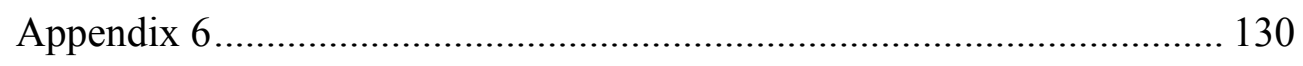

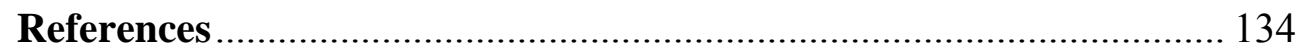

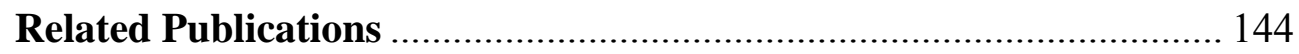




\section{Figure Index}

Fig. 1:

Properties of the different types of pluripotent cells 4

Fig. 2:

The current model for the miRNA biogenesis and function. 9

Fig. 3: Expression levels of miR-19b and miR-20b detected by Real-time PCR in ESCs and maGSCs from different mouse strains . 58

Fig. 4: Expression of Oct-4 and Sox-2 proteins by Western blotting 60

Fig. 5: Expression levels of ESC-specific miRNAs detected by Real-time PCR ... 61

Fig. 6: Expression of pluripotency markers in ESCs and maGSCs from 129/Sv background after culture for 35 days under standard ESC culture conditions (from passage 15 to passage 25) or in Gel+RA.

Fig. 7: Immunostaining for SSEA-1 (Cy-3) in ESCs and maGSCs from 129/Sv background under standard ESC culture conditions (untreated) and after culture for 35 days in Gel+RA

Fig. 8: $\quad$ miRNA expression levels in ESC R1 and maGSC 129SV of passages 15 and 25 64

Fig. 9:

Fig. 10: miRNA expression levels in untreated ESC R1 and maGSC 129SV cells compared with the respective cells cultured for 35 days in Gel+RA .. 65 Cell culture strategy for in vitro differentiation of ESCs and maGSCs from 129/Sv mouse strain (ESC R1 and maGSC 129SV)....

Fig. 11: Cell culture strategy for in vitro differentiation of ECCs (F9 cells)..... 68

Fig. 12: Expression of Oct-4 and Sox-2 proteins by Western blotting in ESCs and maGSCs cultured for five days under the above mentioned conditions

Fig. 13: $\quad$ Expression of Oct-4 protein in untreated maGSCs (FL+LIF/calibrator) and in maGSCs under Gel condition over time (5, 10 and 21 days).

Fig. 14: $\quad$ Expression of Oct-4 mRNA by Real-time PCR in ESCs and maGSCs cultured for 5, 10 and 21 days in all three differentiation conditions compared with untreated cells. 70

Fig. 15: $\quad$ Expression of Oct-4 mRNA by Real-time PCR in F9 cells treated with RA compared with untreated cells

Fig. 16: $\quad$ Expression profiles of members of the miRNA 290 family in ESCs and maGSCs from mouse strain 129/Sv (ESC R1 and maGSC 129SV) under different differentiation conditions after 5, 10 and 21 days in culture compared with untreated cells 
Fig. 17: $\quad$ Expression profiles of members of the miRNA 302 family in ESCs and maGSCs from mouse strain 129/Sv under different differentiation conditions after 5, 10 and 21 days in culture compared with untreated cells

Fig. 18: $\quad$ Expression profiles of members of miRNA families 290 and 302 in untreated F9 cells and in those treated with RA for 25 days

Fig. 19: $\quad$ Expression of Nestin mRNA by Real-time PCR in ESCs and maGSCs cultured for 5, 10 and 21 days in all three differentiation conditions compared with untreated cells. 79

Fig. 20: $\quad$ Expression of Brachyury mRNA by Real-time PCR in ESCs and maGSCs cultured for 5, 10 and 21 days in all three differentiation conditions compared with untreated cells.

Fig. 21: $\quad$ Expression of Hnf4 mRNA by Real-time PCR in ESCs and maGSCs cultured for 5, 10 and 21 days in all three differentiation conditions compared with untreated cells. 81

Fig. 22: $\quad$ Expression of Nkx-2.5 mRNA by Real-time PCR in ESCs and maGSCs cultured for 5, 10 and 21 days in all three differentiation conditions compared with untreated cells.

Fig. 23: $\quad$ Expression of Nestin and Hnf4 in untreated and treated with RA F9 cells . 83

Fig. 24: $\quad$ Relative expression levels (qPCR) of miR-302a and Brachyury in ESC

R1 and maGSC 129SV 85

Fig. 25: $\quad$ Relative expression levels (qPCR) of miR-290 and Oct-4 in ESC R1 and maGSC 129SV 86

Fig. 26: $\quad$ miR-291 expression levels at day 5, 10 and 21 under the three differentiation conditions mentioned in the text

Fig. 27: miR-302b expression levels at day 5, 10 and 21 under the three differentiation conditions mentioned in the text 88

Fig. 28: Comparison of expression levels of members of miRNAs and differentiation markers at day 10 in ESCs and maGSCs from mouse strain 129/Sv between Gel+RA and Gel+RA from day 5 conditions 89

Fig. 29: $\quad$ Expression of Dnmt3A mRNA by Real-time PCR in ESCs and maGSCs cultured for 5, 10 and 21 days in all three differentiation conditions compared with untreated cells. 92 
Fig. 30: $\quad$ Expression of Dnmt3B mRNA by Real-time PCR in ESCs and maGSCs

cultured for 5, 10 and 21 days in all three differentiation conditions

compared with untreated cells.

93

Fig. 31: Genomic organization of miR-17-92, miR-106a-363 and miR-106b-25

miRNA clusters and structures of the respective pri-miRNAs .................. 99

Fig. 32: Genomic organization and structure of miR-290 cluster.......................... 101

Fig. 33: $\quad$ Dppa-3 mRNA expression in ESC R1 and maGSC 129SV during differentiation 110

Fig. 34: Global DNA methylation profile of PGCs during development towards male or female gametes 


\section{Table Index}

Tab. 1: $\quad$ Differentially expressed miRNAs between ESC Stra8 and the respective maGSCs

Tab. 2: $\quad$ Differentially expressed miRNAs between ESC R1 and the respective

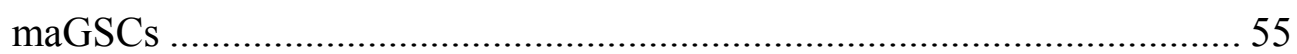

Tab. 3: Differentially expressed miRNAs between maGSC Stra8 and maGSC 129SV 56

Tab. 4: $\quad$ Computational prediction of targeting miRNAs for genes mediating Bmp-4, Wnt and Nodal pathways..... 108 


\section{Abbreviations}

A

$\mathrm{AB}$

ß

BLAST

bp

BSA

$\mathrm{C}$

${ }^{\circ} \mathrm{C}$

cDNA

CIP

$\mathrm{cm}$

dATP

$\mathrm{dCTP}$

$\mathrm{ddH}_{2} \mathrm{O}$

DEPC

dGTP

$\mathrm{dH}_{2} \mathrm{O}$

dil

DNA

DNase

dNTP

dTTP

$\mathrm{ECC}(\mathrm{s})$

E.coli
Purine base adenine

Antibody

Beta

Basic Local Alignment Search Tool

Base pairs

Bovine serum albumin

Pyrimidine base cytosine

Grades Celsius

complementary DNA

Calf Intestinal Phosphatase

Centimeter

Deoxyadenosine triphosphate

Deoxycytidine triphosphate

Double distilled water

Diethylpyrocarbonate

Deoxyguanosine triphosphate

Distilled water

Dilution

Deoxyribonucleic acid

Deoxyribonuclease

Deoxynucleoside triphosphate

Deoxythymidine triphosphate

Embryonic carcinoma cell(s)

Escherichia coli 
EDTA Ethylendiaminetetraacetic acid

EBs

Embryoid bodies

$\mathrm{ESC}(\mathrm{s})$

Embryonic stem cell(s)

EtOH

Ethanol

et al.

et alii (and others)

FL(s)

Feeder layer(s)

G

Purine base Guanine

g

Gram

ICM

Inner cell mass

$\mathrm{IgG}$

Immunoglobulin $\mathrm{G}$

IPTG

Isopropyl- $\beta-D-1-$ thiogalactopyranoside

$\mathrm{kb}$

Kilo base pairs

$\mathrm{kDa}$

Kilodalton

1

Liter

M

Molarity

$\mathrm{m}$

Milli

$\mu$

Micro

$\operatorname{maGSC}(\mathrm{s})$

Multipotent adult germline stem cell(s)

miRNA

MicroRNA

$\mathrm{mM}$

Millimolar

MOPS

3-(N-morpholino)-propanesulfonic acid

mRNA

Messenger-RNA

$\mathrm{n}$

Nano

NCBI

„National Center for Biotechnology

Information"

nt

Nucleotide 
OD

PBS

PCR

PFA

PMSF

RNA

RNase

RT-PCR

RT

SDS

SV40

$\mathrm{T}$

Tris

U

UTR

Vol.

$\mathrm{v} / \mathrm{v}$

WT

X-Gal
Optical density

„Phosphate Buffered Saline“

„Polymerase Chain Reaction“

Paraformaldehyde

Phenylmethanesulfonilfluoride

Ribonucleic acid

Ribonuclease

Reverse-Transcription-PCR

Room temperature

Sodium dodecyl sulfate

Simian Virus 40

Pyrimidine base thymidine

Tris(hydroxymethyl)-aminomethane

Unit

„untranslated region“

Volume

Volume/Volume

Wild type

5-brom-4-chlor-3-indolyl- $\beta-D-$

galactopyranoside 


\section{Introduction}

\subsection{Definitions and general concepts about pluripotent cells}

\subsubsection{Stem cells and their differentiation potential during development}

A stem cell is a cell that has the ability of self-renewal for long periods (in case of adult stem cells for the whole life of an organism) as well as to give rise to the specialized cells that form the tissues and the organs of an organism, a process called differentiation. Based on their differentiation potential, stem cells are classified as totipotent, pluripotent, multipotent or unipotent (Marshak et al. 2001).

A totipotent stem cell has the ability to generate all cell types and tissues and to form an embryo. Totipotent are the zygote and the cells that result from its first divisions (like cells of the 2-cell stage embryo). These cells develop into all embryonic or extra-embryonic tissues (like placenta and umbilical cord).

A pluripotent stem cell can give rise to all types of cells of the three germ layers (ectoderm, mesoderm, endoderm) of an embryo but cannot form a viable organism. From these cells all cells arise that form an organism but not extra-embryonic tissues. Pluripotent cells are a number of cells developed in vitro, like embryonic stem cells (ESCs). Embryonic stem cells are cells isolated from the inner cell mass (ICM) of the embryo, that are further propagated ex vivo under specific culture conditions. ESCs closely resemble the cells of the 
ICM from which they originate. However, whether ESCs and cells of the ICM are the same is a matter of dispute (Brook and Gardner 1997; Pelton et al. 1998).

Finally, multipotent and unipotent stem cells are capable of differentiating along some or only one lineage. This applies to progenitor (precursor) cells or adult stem cells, that are responsible for derivation and steady-state self renewal of a tissue or an organ, and can give rise to cell types only of some tissues or organs (Ralston and Rossant 2005).

An overview of early stages of development as well as differentiation potential of cells from the three germ layers is depicted in Figure 1 of Appendix 1.

\subsubsection{Defining pluripotency}

As mentioned above, pluripotency is defined from the potential of cells to differentiate into all three primary germ layers of the embryo, an ability observed in the cells forming the inner cell mass of the blastocyst. During the last three decades isolation and cultivation of pluripotent cells has been possible from a number of sources and under specific culture conditions. Laboratory-based criteria for testing the pluripotent state of these cells in mouse include three types of experiments: a. Formation of chimeras after injection of these cells into a blastocyst and transfer to the uterus of a pseudopregnant female mouse (and the ability of integrating into all tissues). b. formation of teratomas after injection of these cells into a genetically identical or immunodeficient adult mouse. c. In vitro differentiation into cell types of all three germ layers (Kirschstein and Skirboll 2001). 
In addition, properties of a pluripotent cell include:

- Ability of long-term self-renewal

- Normal karyotype during long term culture

- Ability of colonizing the germ line during the formation of chimeras and giving rise to eggs or sperm cells

- Clonogenic, that is a single cell can give rise to a colony of genetically identical cells, or clones, which have the same properties as the original cell

- Expression of the transcription factor Oct-4

- Lack of the G1 checkpoint in the cell cycle (Kirschstein and Skirboll 2001; Lensch et al. 2006)

In fact, all these are characteristics of ESCs and confirmation or rejection of pluripotency of a cell type is often based on its similarities or differences with ESCs. Thus, often the term pluripotent cells is synonymous with that of ESCs.

\subsubsection{Types of pluripotent cells in mouse}

Based on the source from which pluripotent cells originate, the following types of pluripotent cells have been derived in mouse:

- Embryonic stem cells (ESCs): The 'gold standard' of pluripotent cells, isolated from the ICM of the early blastocyst (Evans and Kaufman 1981).

- Embryonic germ cells (EGCs): Derived from primordial germ cells (PGCs), precursors of the germ cell line in the embryo (Hogan 2001; Rohwedel et al. 1996). 
- Embryonic carcinoma cells (ECCs): Derived from testicular teratocarcinomas. They can be regarded as the malignant counterparts of ESCs (Andrews et al. 2001).

- Multipotent adult germline stem cells (maGSCs): Derived recently from the adult mouse testis. They are extensively described later.

- Induced pluripotent stem cells (iPS cells): Somatic cells reprogrammed to a pluripotent state by ectopic expression of defined factors (Meissner et al. 2007).

Figure 1 and Appendix 1 (Fig. 2 of Appendix 1) depict the different pluripotent cell types, their origin and their main properties.

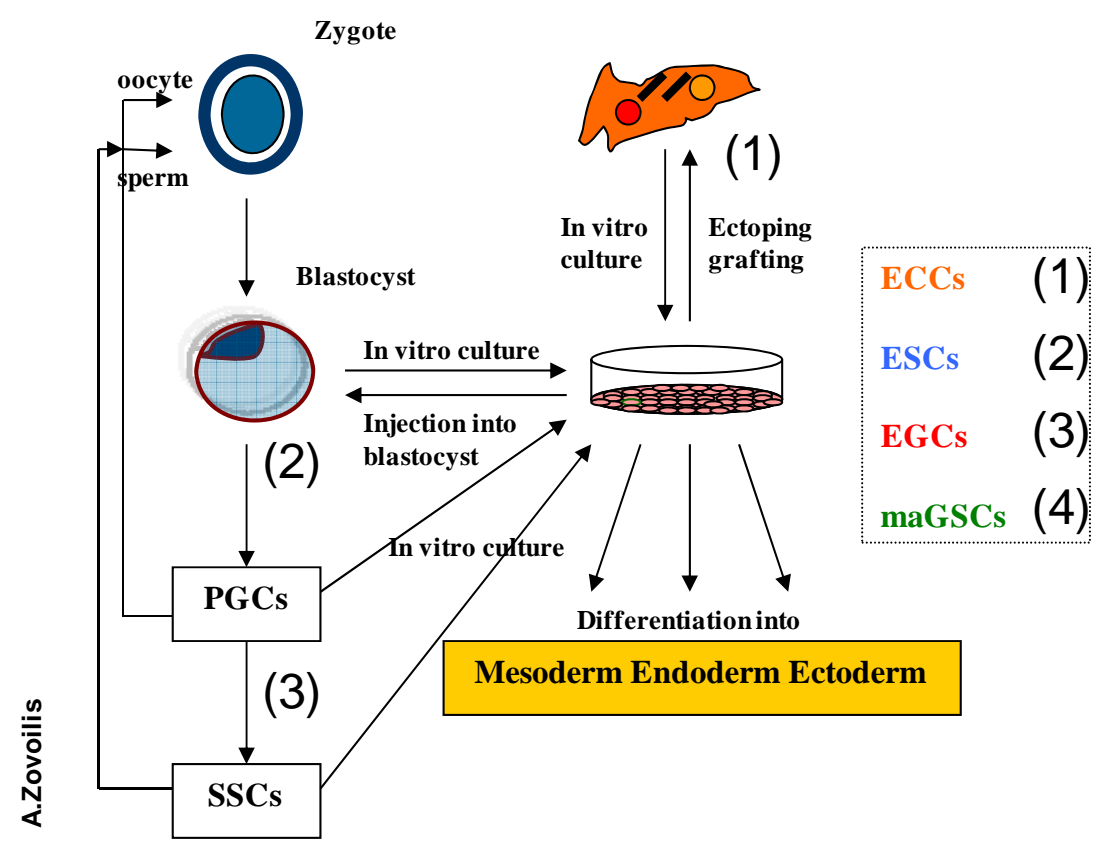

(4)

Fig 1. Properties of the different types of pluripotent cells. The diagram on the left represents the origin of these cells in vivo and their position in developmental time line. The arrows represent in vivo or in vitro processes in which one cell type can develop or be transformed into another. 


\subsection{Multipotent adult germline stem cells in mouse and their potential}

Until recently the only cells apart from ESCs that fulfilled, although partially, the criteria for pluripotency in mouse were EGCs and ECCs. However, ESCs and EGCs face immune reaction after transplantation and there are ethical issues regarding the usage of embryos, while use of ECCs is excluded due to malignant nature of these cells. Thus, identification of an alternative source for pluripotent cells is crucial for use in regenerative medicine.

Several studies during the last five years have revealed that the germline lineage retains the potential to generate pluripotent cells (for a comprehensive review see de Rooij and Mizrak 2008). In 2004, ESC-like cells were found in germ stem cell cultures established from neonatal mouse testis and were designated as multipotent germline stem cells (mGSCs). These ESC-like cells are phenotypically similar to ESCs and EGCs, except in their genomic imprinting pattern. They differentiated into various types of somatic cells in vitro under conditions used to induce the differentiation of ESCs and produced teratomas after inoculation into mice. Furthermore, these ESC-like cells formed germline chimeras when injected into blastocysts (Shinohara et al. 2004).

In 2006, the group of Prof. Engel in Goettingen isolated and cultured for the first time spermatogonial stem cells (SSCs) from the adult mouse testis which respond to culture conditions and acquired ESClike properties (Guan et al. 2006). SSCs were isolated from adult mice transgenic for EGFP under Stra8 promoter (which is active in spermatogonia) and Rosa 26-LacZ. The authors proved that the pluripotency and plasticity of these cells, which were named multipotent adult germline stem cells (maGSCs), is similar to ESCs. 
maGSCs are able to spontaneously differentiate into derivatives of the three embryonic germ layers in vitro, to generate teratomas in immunodeficient mice and to contribute to the development of various organs when injected into an early blastocyst.

Isolation of SSCs and the derivation of maGSCs is not restricted to EGFP+ cells from the transgenic Stra8-EGFP/ROSA26-LacZ mouse (Stra8 mouse). ESC-like cell lines were also successfully derived from testes of four different strains of mice (FVB, C57BL/6, 129/Sv and the transgenic Stra8 mouse) by morphological criteria only. These results were confirmed by other groups (Izadyar et al. 2008; Seandel et al. 2007).

It is not yet clear whether SSCs are pluripotent themselves or generation of maGSCs in vitro may result from genetic reprogramming of SSCs in culture. Interestingly, another group showed recently that a single spermatogonial stem cell can dedifferentiate from a highly lineage-specified state to a pluripotent state (Kanatsu-Shinohara et al. 2008).

The possibility to derive pluripotent stem cells from the adult testis has important implications for germ cell biology and opens the possibility of using these cells for biotechnology and medicine. 


\subsection{Critical pathways controlling pluripotency in mouse}

The most critical pathways regulating self-renewal in mouse ESCs are those mediated by leukaemia inhibitory factor (LIF) and Oct-4. LIF, a member of the LIF-oncostatinM-Il-6 superfamily of cytokines, is necessary for maintenance of feeder-free ESC lines. This activity is mediated through activation of STAT3 and the transcription factor cMyc, which is believed to be a key target of LIF-STAT3 signalling (Matsuda et al. 1999; Niwa et al. 1998)

Equally important for maintaining the ESC state is the octamer motif binding transcription factor Oct-4. ESC lines express high levels of Oct-4 which are required to maintain the ESC state, and are downregulated upon differentiation (Buitrago and Roop 2007; Nichols et al. 1998; Pesce et al. 1999; Schoeler et al. 1989). Oct-4 itself cooperates with other transcription factors to positively and negatively regulate downstream gene expression. The most important are Sox-2 and Nanog. These factors form the core of an extensive transcriptional network that control pluripotency in ESCs, by controlling each other as well as a big number of target genes (Rodda et al. 2005; Pan and Thomson 2007; Masui et al. 2007; Wang et al. 2007) (Fig. 3 of Appendix 1).

In addition, a number of other pathways also occur in ESCs acting in cooperation or in parallel with LIF-STAT3 and Oct-4 pathways like BMP and Wnt pathways, while pluripotency regulators include also heterochronic genes, genes involved in telomeric regulation and polycomb repressors (Liu et al. 2007).

Recently a new group of noncoding RNAs, called miRNAs, has been implicated to play a key role in regulation of pluripotency in mouse ESCs and embryonic development. 


\section{4 microRNAs in pluripotency and development}

\subsection{1 miRNAs overview}

According to the current convention, a mature miRNA is defined as a ssRNA of 22 nucleotides in length which is generated by the RNaseIII-type enzyme Dicer from an endogenous transcript that contains a local hairpin structure.

Two processing steps lead to mature miRNA formation in animals (Fig. 2). Firstly, pri-miRNA (the primary transcript produced by RNA polymerase II) is processed into a $\sim 70$-nucleotide precursor (or multiple precursors in case of miRNA clusters) in the nucleus by an RNase-III enzyme called Drosha (pre-miRNA). After this initial cleavage and export from the nucleus into the cytoplasm by Exportin 5 , each hairpin precursor is cleaved by Dicer into a small dsRNA duplex (miRNA/miRNA*), which finally results into the mature $\sim 22 \mathrm{nt}$ miRNA.

$\sim 50 \%$ of miRNAs are proved or postulated to be transcribed from a single polycistronic pri-miRNA (miRNA clusters), indicating that these miRNAs are co-ordinately regulated during development (Bartel 2004; Bartel and Chen 2004). 


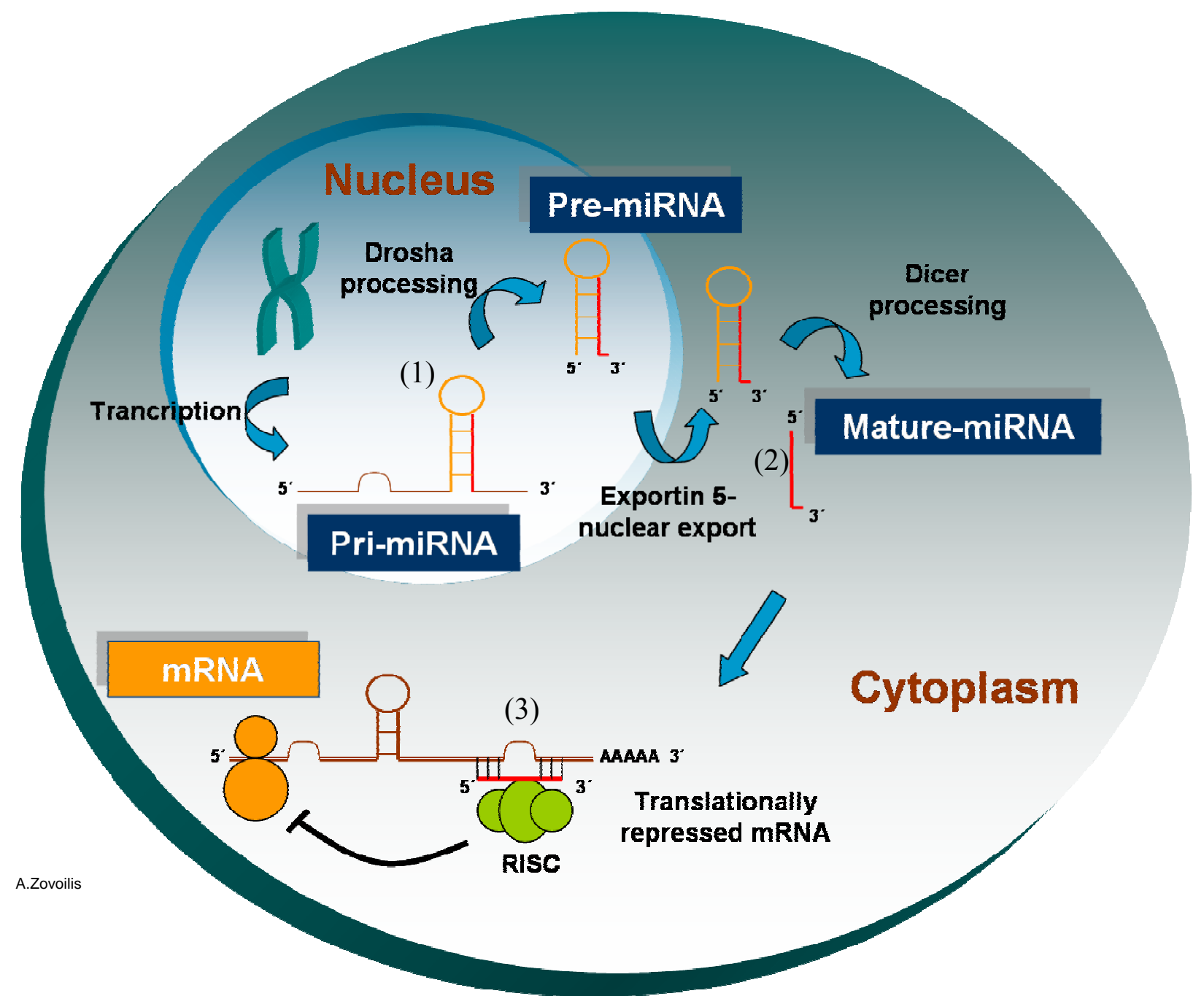

Figure 2. The current model for miRNA biogenesis and function. Blue legends (pri-miRNA, pre-miRNA and mature miRNA) depict the different processing stages leading from the primary transcribed RNA molecule to mature miRNA, whose sequence is shown in red (2). The hairpin RNA structure that enables this processing is depicted in yellow (1). The lower part of the figure shows miRNA mediated translational silencing of target mRNA. Partial sequence complementarity between the miRNA and the 3 'untranslated region (UTR) of the mRNA (3) brings about repression of protein synthesis. RISC, RNA induced silencing complex.

\subsection{2 miRNAs function}

With more than 200 members per species in higher eukaryotes, miRNAs are one of the largest gene families accounting for $\sim 1 \%$ of 
the genome (Kim 2005). The predominant regulatory effect of miRNAs is to repress their target mRNAs. Mechanisms for this include translational repression and mRNA cleavage. The first seems to be the dominant regulatory mode in animals, while the second characterizes plants. It has been shown, that in animals the singlestranded mature miRNA associates with a complex that is similar, if not identical, to the RNA Induced Silencing Complex (RISC) which is known to mediate siRNA silencing. The miRNA/RISC complex represses protein translation by binding to sequences in the $3^{\prime}$ untranslated region of specific mRNAs (Ambros 2004).

Recent studies have shown that miRNAs have key roles in diverse regulatory pathways, including control of developmental timing, hematopoietic cell differentiation, apoptosis, cell proliferation, carcinogenesis and organ development. Regulation of gene expression by miRNAs is realized by formation of complex regulatory networks, in which each miRNA can target many different mRNAs, and conversely, several different miRNAs can cooperatively control a single mRNA target (Esquela-Kerscher and Slack 2006; He L and Hannon 2004; Kim 2005).

It was shown that the expression of certain genes is more dependent on the level of regulatory miRNAs than on the level of mRNAs that encode the proteins (Johnson et al. 2005). Taking into account this fact as well as that over one third of all human genes are predicted to be targeted by miRNAs, it becomes obvious that discovery of miRNAs adds a new dimension to our understanding of complex gene regulatory networks during development (Chen $C$ et al. 2007). Identification of the first mouse ESC specific miRNAs five years ago implicated that the role of miRNAs in the regulation of gene expression may extend also to early embryonic development and pluripotency. 


\subsubsection{Embryonic stem cell specific miRNAs (ESC- specific miRNAs)}

A set of miRNAs has been described to be ESC-specific in mouse, with their expression being repressed during ESC differentiation and undetectable in adult mouse organs. This set of miRNAs consists of miR-290, miR-291a-3p, miR-292-3p, miR-293, miR-294, miR-295 (miR-290 family; Houbaviy et al. 2003) and miR-302a, miR-302b, miR-302c, miR-302d (miR-302 family; Strauss et al. 2006).

In a previous work, miRNAs of the miR-290 family were shown to become repressed in embryoid bodies (EBs) prepared by culturing ES cells for 14 days in either the presence or absence of RA (retinoic acid). It was suggested that their expression is specific for pluripotent ES cells and is either silenced or downregulated upon differentiation (Houbaviy et al. 2003; Houbaviy et al. 2005). Another group confirmed the expression of these miRNAs as well as of those of miR302 family only in ESCs and EBs, and not in somatic tissues. In addition, they reported a negative correlation in EBs between miRNAs of miR-302 family and differentiation time (Strauss et al. 2006; Chen C et al. 2007).

These miRNAs are expressed in clusters (members of each miRfamily are transcribed as parts of the same pri-miRNA) and they have close homologues in human ESCs with the same expression profile during differentiation (Suh et al. 2004). However, their role in pluripotency is still not well defined. 


\subsection{Aims and experiments outline}

The past few years have seen remarkable progress in our understanding and identification of critical conserved pathways regulating self-renewal and pluripotency. However, much of our knowledge about these pathways has come from mouse ESC lines but underlying mechanisms controlling pluripotency in other types of pluripotent cells like maGSCs remain to be elucidated.

The objective of the current study was to test similarities and differences between pluripotent cells derived from adult mouse testes (maGSCs) and embryonic stem cells (ESCs) with respect to microRNAs (miRNAs).

> The first question to be answered was whether ESCs and maGSCs are distinct or equivalent cell types from the viewpoint of global miRNA expression patterns. This question was addressed by the whole miRNA array expression profiling of maGSCs and ESCs and the validation of the results in maGSCs and ESCs from different mouse strains.

> The second question was whether miRNAs previously classified as ESC specific are also expressed in maGSCs and ECCs and thus characterize generally pluripotency. To answer this question ESC and maGSC lines from different mouse strains as well as ECCs of the F9 teratocarcinoma cell line were studied.

$>$ The third question was whether expression of these miRNAs in maGSCs remains like ESCs stable during time and it is restricted only to undifferentiated maGSCs. To answer this question cells were tested after culture for 10 passages (35 days) under 
standard ESC culture conditions and after pluripotency was lost due to differentiation.

The last question was which is the expression profile of these miRNAs during first stages of in vitro differentiation of pluripotent cells and whether there are similarities and differences between ESCs and maGSCs concerning this profile. To answer this, the time-dependent influence of different differentiation factors on this profile was evaluated.

To test whether the observed similarities and differences in miRNA expression are associated with the differentiation state of the cells, results were correlated with time-course and extent of pluripotency and differentiation markers expression.

Finally, results were associated with the pattern of expressed de novo DNA methyltransferases, which have been recently shown to be regulated by these miRNAs (Sinkkonen et al. 2008). 


\section{Materials and Methods}

\section{$2.1 \quad$ Materials}

\subsubsection{Laboratory materials}

\subsubsection{Molecular biology}

14 ml-centrifuge tubes

Coverslips (24 x $60 \mathrm{~mm})$

Disposable filter Minisart NMI

Falcon tubes

Films

Filter tips

Glasware

Hybond $\mathrm{C}$

Microcentrifuge tubes

Microliterpipettes

PCR-tubes

Petri plates

Reaction tubes

Reaction tubes

Real-time pcr plates

Tips without filter

Whatman blotting paper
Corning, New York, USA

Menzel-Gläser, Braunschweig

Sartorius, Göttingen

Schleicher and Schüll, Dassel

Kodak

Biozym, Hessisch Oldendorf

Schott, Mainz

Amersham, Braunschweig

Eppendorf, Hamburg

Pipetman Gilson Abimed,

Langenfeld

Molecular BioProducts, San

Diego, USA

Greiner, Nürtingen

Eppendorf, Hamburg (RNase-free) Brand, Wertheim Applied Biosystems, Darmstadt Eppendorf, Hamburg Schleicher and Schüll, Dassel 


\subsubsection{Cell culture}

Cell culture flasks and plates

Culture slides BD

Cell culture pipettes

Cryocups

Filtering unit

Pasteur pipettes

6 or $10 \mathrm{~cm}$ Plates

Steril Filters

13 ml-centrifuge plastic tubes

$14 \mathrm{ml}$ - centrifuge plastic tubes
Greiner, Nürtingen or Nunc, Wiesbaden

Falcon, Heidelberg Sarstedt, Nümbrecht

Greiner, Nürtingen or Sarstedt, Nümbrecht

Nalgene, Rochester, USA

Brand, Wertheim

Nunc, Wiesbaden

Sartorius, Göttingen

Sarstedt, Nümbrecht

Corning, New York, USA or

Greiner, Nürtingen

\subsubsection{Laboratory equipment}

Accu-Jet

Autoclave

Beckman J2-21 Ultracentrifuge

Beckman JS-7.5 Centrifugerotor

Biophotometer

Branson sonifier 250

Centrifuge Biofuge 13

Centrifuge Heraeus Megafuge 1.0

DNA Sequencer Modell Megabace 1000

Eppendorf Centrifuge 5415 D

Eppendorf Centrifuge 5417 R
Brand, Wertheim

Webeco, Bad Schwartau

Beckman, München

Beckman, München

Eppendorf, Hamburg

Heineman, Schwaebisch

Gmuend

Heraeus, Hanau

Heraeus, Hanau

Amersham, Freiburg

Eppendorf, Hamburg

Eppendorf, Hamburg 
Fluorescence microscope BX60

Freezer VIPseries $-86^{\circ} \mathrm{C}$

GeneAmp PCR System 9600

HT 7900 Analyzer

Incubator IR Autoflow Incusafe

Inverted microscope IX81

Light microscope

Microplate Reader 450

Microwave oven

Molecular Imager FX

MWG Primus 96plus Thermocycler

Neubauer cell chamber

Pipettes

Power supply

PTC-100 Peltier Thermal Cycler

Spectrophotometer Ultraspec 3000

SpeedVac concentrator SVC $100 \mathrm{H}$

Sterilbank HERAsafe

Thermomixer 5436

TurboblotterTM

Ultra low remperature

freezer $-152^{\circ} \mathrm{C}$

UV-Transilluminator

X-Ray Automatic Processor Curix 60 Agfa, München
Olympus, Planegg

Sanyo, Tokyo, Japan

Perkin Elmer, Berlin

Applied Biosystems

Sanyo, Tokyo, Japan

Olympus, Planegg

Zeiss, Göttingen

Bio-Rad, Muenchen

Phillips, Hamburg

Bio-Rad laboratories, München

MWG Biotech, Ebersberg

Schütt Labortechnik, Göttingen

Eppendorf, Hamburg

Gibco BRL, Karlsruhe

Bio-Rad, München

Amersham, Freiburg

Schütt, Göttingen

Heraeus, Hanau

Eppendorf, Hamburg

Schleicher \& Schüll, Dassel

Sanyo, Tokyo, Japan

Herolab, Wiesloch

\subsubsection{Chemicals, biochemical and biological materials}

$1 \mathrm{~kb}$ DNA ladder

Agar

Agarose

Ampicillin

Ampuwa
Invitrogen, Karlsruhe

Difco, Detroit, USA

Invitrogen, Karlsruhe

Sigma, Deisenhofen

Fresenius, Bad Homburg 
$\beta$-Mercaptoethanol

Bisacrylamide

Boric acid

BSA

Chloroform

Coomassie Blue G-250

DAPI

Diethyl pyrocarbonate (DEPC)

Dimethylformamid

Dimethylsulfoxid (DMSO)

Dithiotreitol (DTT)

DMEM

dNTPs

Dye Terminator Mix

EDTA

Ethanol

Ethidium bromide

FCS

Ficoll

Gelatin

Glycerol

Glycine

$\mathrm{HCl}$

HEPES

IPTG

Isopropanol

$\mathrm{KCl}$

L-Glutamine

LIF

Methanol

Milk powder

Mineral oil
Gibco BRL, Eggenstein

Serva, Heidelberg

Sigma, Deisenhofen

Biomol, Hamburg

Baker, Deventer, NL

Sigma, Deisenhofen

Vector Laboratories,

Burlingame, USA

Sigma, Deisenhofen

Sigma, Deisenhofen

Sigma, Deisenhofen

Applichem, Darmstadt

PAN, Aidenbach

Invitrogen, Karlsruhe

Applied Biosystems

Biomedicals, Eschwege

Baker, Deventer, NL

Sigma, Deisenhofen

PAN, Aidenbach

Applichem, Darmstadt

Sigma, Deisenhofen

Invitrogen, Karlsruhe

Biomol, Hamburg

Merck, Darmstadt

Merck, Darmstadt

Biomol, Hamburg

Merck, Darmstadt

Merck, Darmstadt

Gibco BRL, Eggenstein

Chemicon, Temecula, USA

Merck, Darmstadt

Roth, Karlsruhe

Sigma, Deisenhofen 
Mitomycin C

$\mathrm{NaCl}$

$\mathrm{NaOH}$

Non-essential amino acids

NuPAGE MOPS SDS Running Buffer Invitrogen, Karlsruhe

NuPAGE SDS Sample Buffer

Orange $\mathrm{G}$

Paraformaldehyde

PBS

Penicillin/Streptomycin

Peptone

Potassium acetate

Bio-Rad Protein Assay

Restriction enzymes

Retinoic acid

Reverse transcriptase

RNase away

S.O.C medium

SDS

SeeBlue Plus2 Pre-Stained Standart

Tris / $\mathrm{HCl}$

Tris base

TritonX-100

TrypLE Express (Trypsin)

Trypsin / EDTA

Tween 20

Yeast extract

$\mathrm{X}-\mathrm{Gal}$
Sigma, Deisenhofen

Merck, Darmstadt

Merck, Darmstadt

Gibco BRL, Eggenstein

Invitrogen, Karlsruhe

Sigma, Deisenhofen

Merck, Darmstadt

PAN, Aidenbach

PAN-Systems, Nürnberg

Roth, Karlsruhe

Merck, Darmstadt

Bio-Rad, Muenchen

Invitrogen, Karlsruhe

Sigma, Deisenhofen

Invitrogen, Karlsruhe

Biomol, Hamburg

Invitrogen, Karlsruhe

Roth, Karlsruhe

Invitrogen, Karlsruhe

Roth, Karlsruhe

Sigma, Deisenhofen

Roth, Karlsruhe

Gibco BRL, Eggenstein

PAN, Aidenbach

Roth, Karlsruhe

Roth, Karlsruhe

Biomol, Hamburg 


\subsubsection{Solutions and buffers}

\subsubsection{Agarose gel electrophoresis}

$5 \times$ TBE buffer

DNA loading buffer

\author{
$445 \mathrm{mM}$ Tris base \\ $445 \mathrm{mM}$ Boric acid \\ 10 mM EDTA (pH 8)
}

15\% Ficoll 400

$1 \mathrm{mM}$ EDTA

$0.01 \%$ Orange $\mathrm{G}$

$10 \%$ Glycerin

\subsubsection{Frequently used buffers and solutions}

Elution buffer

TE buffer $10 \mathrm{mM}$

Transfer buffer $(1 \mathrm{x})$

Blocking buffer I (B1)

\author{
$1.5 \mathrm{M} \mathrm{NaCl}$ \\ $20 \mathrm{mM}$ Tris/ $\mathrm{HCl}$ (pH 7.5) \\ $1 \mathrm{mM}$ EDTA
}

Tris/ $\mathrm{HCl}(\mathrm{pH} 8.0)$

$1 \mathrm{mM}$ EDTA

$25 \mathrm{mM}$ Tris $\mathrm{pH} 8.3$

$150 \mathrm{mM}$ Glycin

$10 \%$ Methanol

$150 \mathrm{mM} \mathrm{NaCl}$

$10 \mathrm{mM}$ Tris/ $\mathrm{HCl}$ (pH 7.5)

$0,1 \%$ Tween 20

$0,2 \%$ BSA

$0,1 \%$ Tween 20 in PBS 
dNTP-solution (25 mM)

Fixation buffer

IPTG

Protein lysis buffer

P1-buffer

P2-buffer

P3-buffer

X-Gal-solution

DEPC-water
$100 \mathrm{mM}$ dATP

$100 \mathrm{mM}$ dCTP

$100 \mathrm{mM}$ dGTP

$100 \mathrm{mM}$ dTTP

4\% Paraformaldehyde in PBS

$100 \mathrm{mM}$ in $\mathrm{H}_{2} \mathrm{O}$

$10 \mathrm{mM}$ Tris $\mathrm{HCl}(\mathrm{pH} \mathrm{8,0)}$

$1 \mathrm{mM}$ EDTA

$2.5 \%$ SDS

$1 \mathrm{mM}$ PMSF

Proteinase inhibitors

$50 \mathrm{mM}$ Tris/ $\mathrm{HCl}(\mathrm{pH} \mathrm{8,0)}$

$10 \mathrm{mM}$ EDTA

$100 \mu \mathrm{g} / \mathrm{ml}$ RNaseA

$200 \mathrm{mM} \mathrm{NaOH}$

$1 \% \mathrm{SDS}$

3,2 M Potassium acetate (pH5,0)

$2 \% \mathrm{X}-\mathrm{Gal}$ in

Dimethylformamide

$0.1 \%(\mathrm{v} / \mathrm{v})$

Diethylpyrocarbonate 
Gelatin $0.1 \%$

$0.1 \%$ diluted in Ampuwa

water and autoclaved

\subsubsection{Media and cells}

\subsubsection{Eukaryotic cell culture media and cells}

Cell lines:

\begin{tabular}{|l|l|}
\hline Cell line & Reference/Source \\
\hline F9 & ATCC, Manassas \\
\hline NIH/3T3 & $\begin{array}{l}\text { Cell Bank of the Institute of Human Genetics in } \\
\text { Goettingen }\end{array}$ \\
\hline ESC R1 & $\begin{array}{l}\text { Wurst and Joyner 1993 } \\
\text { (kindly provided by Prof. I. Adham in the Institute) }\end{array}$ \\
\hline ESC Stra8 & Kindly provided by Mrs. J. Nolte in the Institute \\
\hline $\begin{array}{l}\text { maGSC } \\
\text { 129SV }\end{array}$ & Guan et al. 2006 \\
\hline maGSC FVB & Guan et al. 2006 \\
\hline maGSC Stra8 & Guan et al. 2006 \\
\hline $\begin{array}{l}\text { maGSC } \\
\text { C57BL }\end{array}$ & Guan et al. 2006 \\
\hline
\end{tabular}

ESC medium
DMEM
0,1 mM non-essential amino- acids
$1 \mathrm{mM}$ Sodium pyruvate
$1 \mu \mathrm{M} \beta$-Mercaptoethanol
2 mM L-Glutamine
$1 \mathrm{mM}$ Penicillin / Streptomycin $20 \%$ FCS
(1, $000 \mathrm{U} / \mathrm{ml} \mathrm{LIF})$ 
FB medium

DMEM

2 mM L-Glutamine

$1 \mathrm{mM}$ Penicillin / Streptomycin $10 \%$ FCS

FB freezing medium

$30 \%$ FB-Medium

$20 \%$ DMSO

$50 \%$ FKS

ESC freezing medium

30\% ESC-Medium

$20 \%$ DMSO

$50 \%$ FKS

\subsubsection{Bacteria culture media and cells}

Bacteria:

Escherichia coli DH5 $\alpha$

Invitrogen, Karlsruhe

LB-medium (pH 7,5)

Peptone $5 \mathrm{~g}$

Yeast extract 2,5 g

$\mathrm{NaCl} 5 \mathrm{~g}$

$500 \mathrm{ml} \mathrm{H} 2 \mathrm{O}$

S.O.C.-medium

Invitrogen, Karlsruhe

For selection

Ampicillin (0,1 mg / ml) 
For Oja-Plates

$100 \mu \mathrm{M}$ IPTG

$0,4 \%$ X-Gal

\subsubsection{Vectors}

pGEM-T easy, Promega, Madison, USA

\subsubsection{Enzymes (with supplied buffers)}

Restriction enzymes

Platinum Taq DNA polymerase

\subsubsection{Antibodies}

$\alpha$-Tubulin

Anti-rabbit and anti-mouse

IgG-Peroxidase antibodies

Oct-4

Sox-2

SSEA-1
Invitrogen, Karlsruhe

Invitrogen, Karlsruhe
Sigma-Aldrich, T5168

Sigma-Aldrich

Abcam, Cambridge, ab19857

Abcam, Cambridge, ab 15830

ES Cell Characterization Kit, Chemicon

Anti-rabbit IgG Cy3-conjugated antibody Sigma-Aldrich 


\subsubsection{Oligonucleotides}

$\begin{array}{ll}\text { Primer name } & \text { Sequence } \\ \text { mVimentin-F } & \text { 5-TGCAGTCATTCAGACAGGATGT-3 } \\ \text { mVimentin-R } & \text { 5-ATCTCTTCATCGTGCAGTTTCTTC-3 } \\ \text { mHNF4-F } & \text { 5-CCACATGTACTCCTGCAGGTTTAG-3 } \\ \text { mHNF4-R } & \text { 5-CGCTCATTTTGGACAGCTTC-3 } \\ \text { mNestin-F } & \text { 5-CTGCAGGCCACTGAAAAGTT-3 } \\ \text { mNestin-R } & \text { 5-ATTAGGCAAGGGGGAAGAGA-3 } \\ \text { mNkx2.5-F } & \text { 5-CCCAAGTGCTCTCCTGCTTTCC-3 } \\ \text { mNkx2.5-R } & \text { 5-GTCCAGCTCCACTGCCTTCTG-3 } \\ \text { mBrachyury-F } & \text { 5-CAATGGAGGGGGACAGATCAT-3 } \\ \text { mBrachyury-R } & \text { 5-AAGGGCTGTAATCTCCTCATTCTG-3 } \\ \text { mDNMT3A2-F } & \text { 5-AGGGGCTGCACCTGGCCTT-3 } \\ \text { mDNMT3A2-R } & \text { 5-TCCCCCACACCAGCTCTCC-3 } \\ \text { mDNMT3B1/B6-F } & \text { 5-TGGGATCGAGGGCCTCAAAC-3 } \\ \text { mDNMT3B1/B6-R } & \text { 5-TTCCACAGGACAAACAGCGG-3 } \\ \text { mSdha-F } & \text { 5-GCTTGCGAGCTGCATTTGG-3 } \\ & \text { 5-CATCTCCAGTTGTCCTCTTCCA-3 } \\ & \\ & \end{array}$

\subsubsection{Kits}

Chemiluminescence kit

Dyenamic ET-Terminator Mix

ES cell characterization kit
Santa Cruz Biotech., Santa Cruz, USA

Amersham, Freiburg

Chemicon, Temecula,USA 
miRCURY'TM LNA microRNA

Array microarray kit and power

labeling kit

Exiqon, Vedbaek

miRNeasy mini Kit

Qiagen, Hilden

miScript Primer Assays

Qiagen, Hilden

miScript Reverse Transcription Kit

Qiagen, Hilden

miScript SYBR Green PCR Kit

Qiagen, Hilden

Montage PCR

Millipore, Karlsruhe

NuPAGE Pre-Cast Gel System

Invitrogen, Karlsruhe

pGEM®T-easy Vector System I

Promega, Madison, USA

QIAGEN Plasmid Mini Kit

Qiagen, Hilden

Qiaquick Gel Extraction Kit

Qiagen, Hilden

QuantiTect SYBR-Green PCR

Master mix

Qiagen, Hilden

\subsection{Animal cell culture methods}

\subsubsection{General animal cell culture procedures and conditions}

During working in the animal cell culture lab all rules regarding aseptic technique and "Good Cell Culture Practice" were followed, to 
ensure all cell culture procedures are performed to a standard that will prevent contamination from bacteria, fungi and mycoplasma and cross-contamination with other cell lines. All cells were cultured in the appropriate culture flasks or plates in an incubator at $37^{\circ} \mathrm{C}$ in a humidified atmosphere of $5 \% \mathrm{CO} 2$. Cells were grown to $80 \%$ confluence.

\subsubsection{Thawing of frozen cell lines, subculture and cryopreservation}

All cell lines that were used were obtained frozen from the institute's culture collection. For revitalization, frozen cells were quickly thawed at $37^{\circ} \mathrm{C}$ water bath, gently transferred to disposable Falcon tubes containing $7 \mathrm{ml}$ growth medium and spun down as described below. Supernatant was discarded by aspiration and cells were plated out after being resuspended in a suitable amount of prewarmed growth medium.

Before confluence was reached, cells were subcultured. Cells were washed once with sterile PBS and incubated in minimal amount of trypsin solution (TrypLETM Express) at $37^{\circ} \mathrm{C}$ until they had detached from the dish (5-10 min). The process was controlled under an inverted microscope. Trypsin activity was inhibited by addition of growth medium in which the cells were subsequently resuspended. $100-200 \mu 1$ were removed and cell counting was performed, when necessary, using an improved Neubauer chamber. Then the cells were plated out or harvested for cryopreservation.

For subculture resuspended cells were spun down $(1,000 \mathrm{xg}$ for $5 \mathrm{~min}$ at RT). The supernatant was aspirated and the cells were resuspended in the suitable amount of prewarmed medium. Then the required number of cells was transferred to new labeled flasks containing pre- 
warmed medium (usually to a dil 1:3-1:4 of the initial cell number). For cryopreservation, after trypsinization, resuspended cells were spun down (1,000xg for $5 \mathrm{~min})$. The supernatant was aspirated and the cells were resuspended in ice cold freezing medium. Cells were kept for 7 days at $-80^{\circ} \mathrm{C}$, and then stored in liquid nitrogen.

\subsubsection{Cell quantification}

Under sterile conditions 100-200uL of cell suspension were removed and an equal volume of trypan blue was added. Both sides of the chamber were filled $(10 \mu \mathrm{l}$ each) with cell suspension and viewed under a light microscope using x20 magnification. The number of viable cells (seen as bright cells) and non-viable cells (stained blue) was counted. The concentration of viable and non-viable cells and the percentage of viable cells was calculated using the equations provided by the haemocytometer manufacturer.

\subsubsection{Preparation, maintenance and cryopreservation of MEFs and feeder layers}

Preparation of MEFs was done as follows: Pregnant mice at day 13.515.5 p.c. where sacrificed. Using sterile forceps and scissors the abdominal cavity was opened and both uterine horns were dissected and placed in a Petri dish. After excess fat and mesentery were trimmed away, horns were opened under microscope and the conceptuses were released and transferred into a fresh dish containing PBS. Using forceps the embryonic membranes were opened, head was pinched off and internal organs were removed. The remaining was transferred into a conical glass tube containing small glass balls and $0.125 \%$ trypsin $/ 0.01 \%$ EDTA and incubated with magnetic agitation at $37{ }^{\circ} \mathrm{C}$ for $20-40$ min. Then FB medium was added to stop 
trypsinization and cells were centrifuged for 10 min with 1,000 rpm. Pellet was resuspended in FB medium and cells were subsequently plated.

When these flasks of primary MEFs were confluent, cells were passaged one more time before freezing and stored under liquid nitrogen in FB freezing medium. When needed cells were thawed and expanded for four more passages in gelatin coated flasks before inactivation. Inactivation was performed with $50 \mu \mathrm{l}$ of $1 \mathrm{mg} / \mathrm{ml}$ mitomycin $\mathrm{C}$ stock solution in $5 \mathrm{ml} \mathrm{FB}$ medium for 2-3 hrs. Then cells were cleaned with PBS and passaged into new gelatin coated flasks for direct use as feeder layers (FLs) after one day.

\subsubsection{Culture of ESCs and maGSCs}

After thawing of the frozen cells in ESC medium on feeder layers, cells were expanded for 1-2 passages before beginning of the experiments. Flasks were seeded as follows: T25 flask, 1-1.5 x $10^{6}$ cells, 6 well plates, $4-5 \times 10^{4}$ cells. For preservation in an undifferentiated state cells were cultured on feeder layer (FL) in ESC medium in the presence of $1,000 \mathrm{U} / \mathrm{ml}$ recombinant mouse leukaemia inhibitory factor (LIF). Undifferentiated phenotype of the colonies was monitored daily in the microscope.

For the differentiation studies, the following monolayer culture conditions were used:

A. ESC medium with FL and LIF (FL+LIF), this is the standard ESC culture condition for maintenance of undifferentiated cells (untreated cells) ;

B. ESC medium with FL, LIF and retinoic acid (RA; 10 ${ }^{-6} \mathrm{M}$ ) $(\mathrm{FL}+\mathrm{LIF}+\mathrm{RA})$; 
C. Cells were cultured in $0,1 \%$ gelatin coated culture flasks with ESC medium, without LIF (Gel);

D. Cells cultured in $0,1 \%$ gelatin coated culture flasks with ESC medium, without LIF but with RA $10^{-6} \mathrm{M}(\mathrm{Gel}+\mathrm{RA})$.

\subsubsection{Culture of F9 cells}

F9 cells were obtained from ATCC (Manassas, USA) and cultured as described previously (Nayernia et al., 2004) in FB medium in gelatin coated flasks.

\subsubsection{Culture of NIH/3T3 cells}

$\mathrm{NIH} / 3 \mathrm{~T} 3$ cells were cultured in FB medium in gelatin coated flasks.

\subsection{RNA isolation}

Plasticware or glassware was used after first eliminating possible RNase contamination. The isolated RNA was stored at $-80^{\circ} \mathrm{C}$.

\subsubsection{Isolation of total RNA including miRNAs}

The common RNA purification protocols do not recover such small RNA molecules like miRNAs. For this reason, the miRNeasy Mini Kit was used for purification of total RNA, including miRNAs and other small RNA molecules. The method used by this kit combines phenol/guanidine-based lysis of samples and silicamembrane-based 
purification of total RNA. The lysis reagent which is included in the kit is a monophasic solution of phenol and guanidine thiocyanate, designed to facilitate lysis of tissues, to inhibit RNases, and also to remove most of the cellular DNA and proteins from the lysate by organic extraction. The maximal amount of starting material was determined based on manufacturer's recommendations, so that the RNA binding capacity of the column is not exceeded. For this reason not more than $5 \times 10^{6}$ cells or $30 \mathrm{mg}$ of tissue were used. Cells were trypsinized and counted, and after washing $1 \mathrm{x}$ with PBS cells were collected as pellet and homogenized by vortexing in $700 \mu \mathrm{l}$ lysis reagent (provided with the kit). The disruption and homogenization of tissues was carried out with mortar and pestle in the same amount of lysis reagent.

After addition of $140 \mu \mathrm{l}$ chloroform, the homogenate was separated into aqueous and organic phases by centrifugation at $12,000 \mathrm{x}$ g for 15 min at $4^{\circ} \mathrm{C}$. RNA partitions to the upper, aqueous phase, while DNA partitions to the interphase and proteins to the lower, organic phase or the interphase. The aqueous phase was extracted, and ethanol (1.5 volumes) was added to provide appropriate binding conditions for all RNA molecules from 18 nucleotides (nt) upwards. The sample was then applied to the RNeasy Mini spin column, where the total RNA binds to the membrane and phenol and other contaminants are efficiently washed away with the provided RWT and RPE buffers according to manufacturer's protocols. RNA was then eluted in $30 \mu \mathrm{l}$ RNase-free water. To obtain a higher total RNA concentration, a second elution step was performed by using the first eluate.

\subsubsection{Determination of nucleic acid concentration}

The concentration of nucleic acids was determined spectrophotometrically by measuring absorption of the samples at 260 
nm. The quality of nucleic acids i.e. contamination with salt and protein was checked by measurements at 260, 280, and 320nm. The concentration was calculated according to the formula:

$\mathrm{C}=(\mathrm{E} 260-\mathrm{E} 320) \mathrm{fc}$

$\mathrm{C}=$ concentration of sample $(\mu \mathrm{g} / \mu \mathrm{l})$

E $260=$ ratio of extinction at $260 \mathrm{~nm}$

E $320=$ ratio of extinction at $320 \mathrm{~nm}$

$\mathrm{f}=$ dilution factor

$\mathrm{c}=$ concentration (standard) / absorption (standard)

for RNA : $\mathrm{c}=0.04 \mu \mathrm{g} / \mu \mathrm{l}$

microRNA array experiments

For microRNA Array experiments the miRCURYTM LNA microRNA Array microarray kit (Exiqon) was used together with the miRCURYTM LNA microRNA Array power labeling kit (Exiqon). Hybridizations and scanning of the slides were carried out in the special equipped ozone free laboratory of the Microarray Facility of University of Goettingen. Experiment design, image acquisition and quantification was performed in cooperation with Dr. Salinas and Mr. Opitz of this lab.

\subsubsection{Labeling of RNA molecules}

$1 \mu \mathrm{g}$ of total RNA containing small RNAs (and miRNAs) was used per labeling reaction per slide hybridization according to manufacturer's recommendations. Prior to labeling integrity of the RNA was assessed on an Agilent Bioanalyzer (RIN values in Figure 4 of Appendix 2). The miRCURYTM LNA microRNA Array Power labeling kit was applied for labeling RNA molecules with a single 
fluorophore per molecule following a 2-step protocol provided by the manufacturer. The first step includes a Calf Intestinal Alkaline Phosphatase for removal of 5'-phosphates from terminal of the microRNAs (CIP treatment). In the second step, a fluorescent label is attached enzymatically to the 3'-end of the miRNAs in the total RNA sample. This is followed by an enzyme inactivation step after which the sample is ready for hybridization. In all experiments a dual color hybridization with $\mathrm{Hy} 3^{\mathrm{TM}}$ and $\mathrm{Hy} 5^{\mathrm{TM}}$ labeled RNA was performed, in which each CIP treated RNA sample was labeled with either Hy $3^{\mathrm{TM}}$ or $\mathrm{Hy}^{\mathrm{TM}}$ (dyes are equivalent to $\mathrm{Cy}-3$ and $\mathrm{Cy}-5$ dyes).

To assure optimal labeling and hybridization, spike-in control probes (Spike-in miRNA kit) supplied in the miRCURYTM LNA microRNA Array microarray kit were used. The miRCURYTM LNA microRNA Array Spike-in kit contains 10 different synthetic unlabeled miRNAs in different concentrations. The set was spiked into each RNA sample prior to labeling so that the synthetic Spike-in miRNAs would hybridize to corresponding capture probes on the miRCURYTM LNA microRNA Array. Spike-in miRNAs are provided at concentrations compatible with endogenous miRNA expression levels aimed at spanning the whole intensity range of miRNAs in most tissue samples. The corresponding capture probes are printed once in every subgrid, thus 32 times each.

The spike-in miRNAs were added in equal amounts to labeling reactions before the dual-color array hybridizations, so that the signals from the spike-in capture probes could be used as a control of the labeling reaction and hybridization and as a help in deciding scanner settings between channels. In addition they were used as a control of the data normalization procedure, to estimate the variance of replicated measurements within arrays and to assess technical variability between different parts of the array. 


\subsubsection{Hybridization and washing of the arrays}

Each Hy3 labeled sample was mixed with a Hy5 labeled sample, denatured and then hybridized on a microarray slide according to the following schema:

$\underline{\text { Slide No }}$

$\begin{array}{lrrrrrr}\text { Cy3 } & \text { Cy5 } & \text { A } & \text { B } & \text { C } & \text { D } \\ \text { we01 } & 1 & -1 & 1 & 0 & 0 & -1 \\ \text { we02 } & 1 & -1 & 0 & 0 & 1 & -1 \\ \text { we03 } & 1 & -1 & 0 & 1 & -1 & 0 \\ \text { we04 } & 1 & -1 & 1 & -1 & 0 & 0 \\ \text { we05 } & 1 & -1 & -1 & 1 & 0 & 0 \\ \text { we06 } & 1 & -1 & 0 & -1 & 1 & 0 \\ \text { we07 } & 1 & -1 & 0 & 0 & -1 & 1 \\ \text { we08 } & 1 & -1 & -1 & 0 & 0 & 1\end{array}$

RNA from:
A: ESC Stra8 (only in Exp A)
B: maGSC Stra8 (only in Exp A)
C: maGSC 129SV
D: ESC R1

Design layout of the experiments, with arrows representing hybridizations, is also shown in the results section.

The miRCURYTM LNA microRNA Array layout was as follows: 
The array was located on a standard size slide as illustrated in the drawing below ( $25.4 \mathrm{~mm}$ by $76.2 \mathrm{~mm}$ or 1 in by $3 \mathrm{in}$ ):

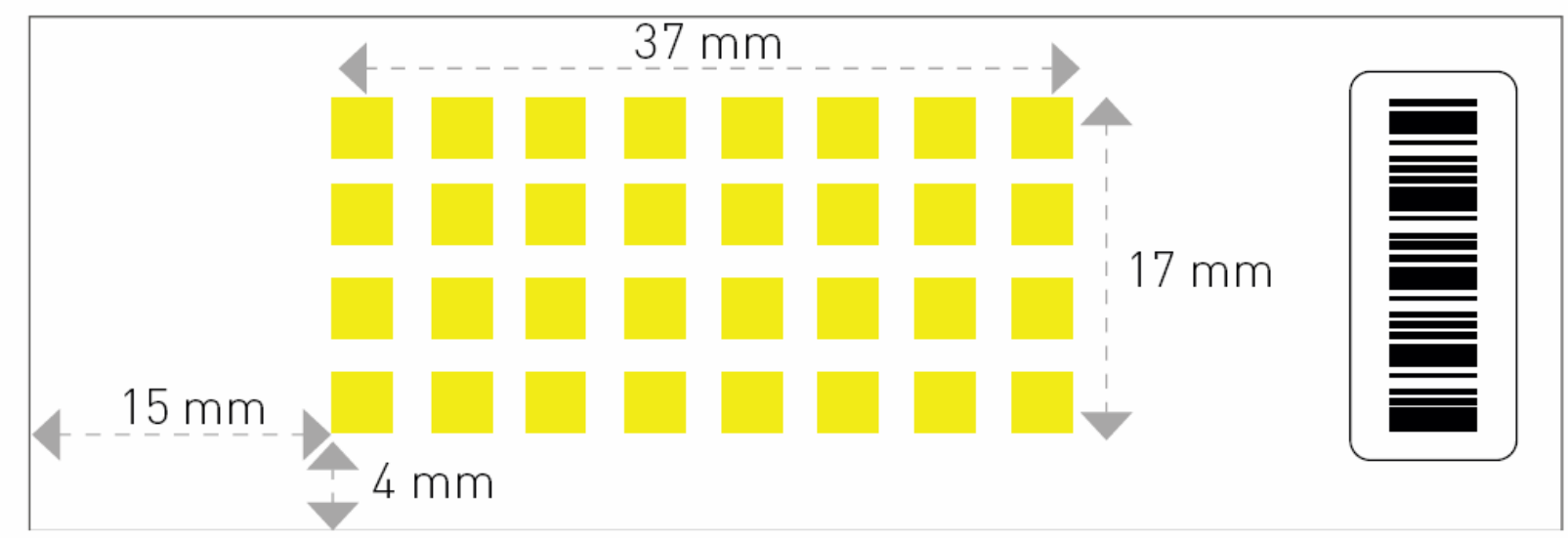

(Source: Exiqon website, www.exiqon.com)

The array carries the following specifications:

- Outer dimensions: $17 \mathrm{~mm}$ wide by $37 \mathrm{~mm}$ long

- Coordinates of first spot on slide $=4 \mathrm{~mm}, 15 \mathrm{~mm}$

- 8 sub-arrays in 4 replicates

- Spot size: $90 \mu \mathrm{m}$

- Distance between spots: $225 \mu \mathrm{m}$

The slides contained capture probes for all miRNAs in human, rat and mouse as annotated in miRBase release 11.0 . In addition, a number of capture probes are available for detection of human miRNAs not included in miRBase (miRPlus ${ }^{\mathrm{TM}}$ probes).

In addition, thirty control capture probes were included in the probe set as follows:

1. Ten spike-in control probes to assure optimal labeling and hybridization (mentioned above).

2. Eight negative capture probes.

3. Twelve capture probes are included that hybridize to small nuclear RNAs. 
Hybridization was carried out manually using an Agilent hybridization SureHyb chamber kit and gasket slide kit in hybridization buffer supplied with the Kit at $56^{\circ} \mathrm{C}$ for $16 \mathrm{hrs}$ in a hybridization oven with rotation. Washing was carried out the next day according to manufacturer's recommendations and then slides were dried and scanned. For image acquisition, quantification, normalization of the results and statistical analysis the software packages provided by the Facility were used. This part of the experiment was carried out in cooperation with the bioinformatician of the Facility, Mr. L. Opitz.

\subsection{RNA and microRNA expression analysis (Reverse Transcription PCR)}

Reverse Transcription PCR (RT-PCR) is a technique, which generates cDNA fragments from RNA templates, and thereafter amplifies them by PCR. It is very useful to determine the expression of genes in specific tissues or in different development stages. In the first step of RT-PCR, called the "first strand reaction", complementary DNA is made from a messenger RNA template using dNTPs and an RNAdependent DNA polymerase (reverse transcriptase) through the process of reverse transcription. RT-PCR exploits a characteristic of mature mRNAs known as the 3' polyadenylated region, commonly called the poly(A) tail, as a common binding site for poly(T) DNA primers. 


\subsubsection{Conversion of miRNAs and other small RNAs into cDNA}

Unlike mRNAs, miRNAs are not polyadenylated in nature. During the reverse transcription step, miRNAs are polyadenylated by poly(A) polymerase. Reverse transcriptase converts RNA (including miRNA, other small noncoding RNA, and mRNA) to cDNA using oligo-dT and random primers. Polyadenylation and reverse transcription are performed in parallel in the same tube. The miScript Reverse Transcription Kit was used which includes miScript Reverse Transcriptase Mix and miScript RT Buffer. miScript Reverse Transcriptase Mix is an optimized blend of enzymes comprising a poly(A) polymerase and a reverse transcriptase. miScript RT Buffer has been developed specifically for use with miScript Reverse Transcriptase Mix. This buffer system enables maximum activity of both enzymes. miScript RT Buffer also contains $\mathrm{Mg}^{2+}$, dNTPs, oligo$\mathrm{dT}$ primers and random primers.

One $\mu \mathrm{g}$ of total RNA containing miRNAs was mixed with $4 \mu \mathrm{l}$ of miScript RT Buffer and $1 \mu \mathrm{lmiScript}$ Reverse Transcriptase Mix, 10 $\mathrm{mM}$ dNTPs in a total volume of $20 \mu \mathrm{l}$. Samples were further incubated at $37^{\circ} \mathrm{C}$ for $60 \mathrm{~min}$ for the first strand cDNA synthesis. Next, the reaction was inactivated by heating at $95^{\circ} \mathrm{C}$ for $15 \mathrm{~min}$ and stored at $20^{\circ} \mathrm{C}$.

\subsubsection{PCR}

Polymerase chain reaction (PCR) is a method that allows logarithmic amplification of short DNA sequences within a longer double stranded DNA molecule. After several (often about 40) rounds of amplification, the PCR product is analyzed on an agarose gel and is abundant enough to be detected with an ethidium bromide stain. For 
reasons whose explanation is beyond the scope of this work, this method of analysis is at best semi-quantitative and, in many cases, the amount of product is not related to the amount of input DNA making this type of PCR a qualitative tool for detecting the presence or absence of a particular DNA. In order to measure messenger RNA (mRNA), the method was extended using reverse transcriptase to convert mRNA into complementary DNA (cDNA) which was then amplified by PCR and again analyzed by agarose gel electrophoresis. In many cases this method has been used to measure the levels of a particular mRNA under different conditions, but the method is actually even less quantitative than PCR of DNA because of the extra reverse transcriptase step. However, in this work this type of PCR was used preliminary to establish the optimal conditions and identify the right positive controls used later in qPCR.

\section{$\underline{\text { Semi-quantitative (regular) RT-PCR }}$}

The amplification reactions were performed in automatic thermocyclers. The PCR reaction contains in general the following substances: 1 ng-10ng template DNA, $1 \mu$ forward primer $(10 \mathrm{pmol} /$ $\mu \mathrm{l}), 1 \mu \mathrm{l}$ reverse primer $(10 \mathrm{pmol} / \mu \mathrm{l}), 1 \mu \mathrm{l} 10 \mathrm{mM}$ dNTPs, $5 \mu \mathrm{l} 10 \mathrm{x}$ PCR buffer, $1.5 \mu 150 \mathrm{mM} \mathrm{MgCl}_{2}$ and $1 \mu \mathrm{Taq}$ DNA polymerase (5 $\mathrm{U} / \mu \mathrm{l})$. The reaction mix was made up to $50 \mu \mathrm{ldd} \mathrm{H}_{2} \mathrm{O}$ and placed in a $200 \mu \mathrm{l}$ reaction tube and placed in thermocycler. A standard PCR program is as follows: Initial denaturation $95^{\circ} \mathrm{C}$ for $5 \mathrm{~min}$ followed by 30-35 cycles of: $95^{\circ} \mathrm{C}$ for $30 \mathrm{sec}$ (denaturation), $55^{\circ} \mathrm{C}-65^{\circ} \mathrm{C}$ for 40 sec (annealing, temperature depends on primer's $\mathrm{Tm}$ value), $72^{\circ} \mathrm{C}$ for (extension, time depends on the PCR product, as 1 min for $1 \mathrm{~kb}$ DNA). Then a final extension step followed at $72^{\circ} \mathrm{C}$ for $10 \mathrm{~min}$ and then a step at $4^{\circ} \mathrm{C}$ for infinite time till the PCR tubes were taken from the PCR machine. The simplest formula for calculating the $\mathrm{Tm}$ (melting temperature) of the primers is: $\mathrm{Tm}=4^{\circ} \mathrm{C} \times$ (number of $\mathrm{G}$ 's and C's in the primer) $+2^{\circ} \mathrm{C} x$ (number of A's and T's in the primer). 


\subsubsection{Real-time RT-PCR (qPCR) for miRNAs}

In contrast to regular RT-PCR and analysis by agarose gels, Real-time RT-PCR or quantitative PCR (qPCR) allows accurate quantification of starting amounts of cDNA targets. In Real-time PCR using SYBR green binding to amplified cDNA, we are simply measuring the fluorescence increase as the dye binds to the increasing amount of DNA in the reaction tube. SYBR Green I binds to all double-stranded DNA molecules, emitting a fluorescent signal of a defined wavelength on binding. The excitation and emission maxima of SYBR Green I are at $494 \mathrm{~nm}$ and $521 \mathrm{~nm}$, respectively, and are compatible for use with any Real-time cycler. Detection takes place in the extension step of Real-time PCR. Signal intensity increases with increasing cycle number due to the accumulation of PCR product.

The cDNA converted from total RNA containing miRNAs served as the template for Real-time PCR analysis using the miScript Primer Assay in combination with the miScript SYBR Green PCR Kit. The oligo-dT primers used in the reverse transcription step have a universal tag sequence on the $5^{\prime}$ end. In the Real-time PCR step miRNAs are amplified using the miScript Universal Primer, which primes from this universal tag sequence, together with the miRNAspecific primer (the miScript Primer Assay). A single cDNA preparation is sufficient to interrogate multiple miRNAs by Real-time PCR using different miRNA-specific primers.

In this study miScript Primer Assays (available from Qiagen) for the following miRNAs were used:

290_5p, 291_3p, 292_3p, 293, 294, 295, 302a, 302b, 302c, 302d, 19b, $20 \mathrm{~b}$.

Real-time RT-PCRs were performed in the respective cycler. The PCR reaction contained: $1 \mu \mathrm{l}$ cDNA , $5 \mu \mathrm{l} 2 \mathrm{x}$ miScript SYBR Green 
PCR Master Mix, $1 \mu \mathrm{l}$ 10x miScript Universal Primer, $1 \mu 1$ 10x miScript Primer Assay and RNase free water to a total volume of 10 $\mu \mathrm{dd} \mathrm{H}_{2} \mathrm{O}$ and placed into the individual wells of a 384-well plate. The PCR program was as follows: $50^{\circ} \mathrm{C}$ for $2 \mathrm{~min}$, initial activation step $95^{\circ} \mathrm{C}$ for $15 \mathrm{~min}$ followed by 40 cycles at $94^{\circ} \mathrm{C}$ for $15 \mathrm{sec}$ (denaturation), $55^{\circ} \mathrm{C}$ for $30 \mathrm{sec}$ (annealing) and $70^{\circ} \mathrm{C}$ for $30 \mathrm{sec}$ (extension). Then a final dissociation curve (melting curve) was made, and PCR plates were kept at $4{ }^{\circ} \mathrm{C}$ till they were taken out from the PCR machine.

However, nonspecific PCR products and primer-dimers will also contribute to the fluorescent signal. Therefore, high PCR specificity is required when using SYBR Green I. A way to check that the correct fragments were amplified is to do a melting curve. After Real-time PCR amplification, the machine was programmed to do a melting curve, in which the temperature is raised by a fraction of a degree and the change in fluorescence is measured. At the melting point, the two strands of DNA will separate and the fluorescence rapidly decreases. The software plots the rate of change of the relative fluorescence units (RFU) with time (T) $(-\mathrm{d}(\mathrm{RFU}) / \mathrm{dT})$ on the Y-axis versus the temperature on the $\mathrm{X}$-axis, and this will peak at the melting temperature (Tm). If the peaks are not similar, this might suggest contamination, mispriming, primer-dimer artifacts etc. A primerdimer artifact would give a peak with a lower melting temperature (because it is such a short DNA).

PCR specificity was checked i) by the melting curves mentioned above, ii) by gel electrophoresis and iii) by sequencing of the PCR products after gel extraction and cloning into a pGEM®T-easy Vector, which is mentioned below (representative results are shown in Figures 1-3 of Appendix 2). Based on these preliminary results, it was decided not to include miR-302c in this study, since the high amount 
of unspecific products observed for this miRNA could not guarantee reliability of the results.

\section{Analysis of data}

Gene expression levels are calculated by determining the ratio between the amount of a target gene and an endogenous reference gene that is in all samples. This ratio is then compared between different samples. Usually, housekeeping or maintenance genes are chosen as endogenous reference. The target and reference gene are amplified separately from the same sample.

The normalized value is determined for each sample and can be used to compare differential expression of a gene in different tissues. In this work relative quantification was carried out as follows:

- RNU6B, whose expression level does not change under the experimental conditions or between different tissues was chosen as endogenous reference.

- A dilution series of a cDNA control sample (ESC R1) was prepared to construct standard curves for target and reference genes.

- CT values for the standards and samples of interest were determined. -Standard curves for both target and reference were constructed by plotting CT values (Y-axis) against the $\log$ of template amount or dilution (X-axis).

- Amount of target and reference in samples of interest were calculated using $\mathrm{CT}$ value and corresponding standard curve.

- The normalized amount of target was calculated by dividing the amount of target by the amount of reference (because replicate reactions were performed, average values were used).

- The normalized target amount of one sample was set to 1 (calibrator sample) and relative expression level of target gene in samples of 
interest was compared by dividing the normalized target amounts by the value of the calibrator.

\subsubsection{Real-time RT-PCR (qPCR) for mRNAs}

qRT-PCR for mRNAs was performed as for miRNAs with the following modifications: $5 \mu \mathrm{l} 2 \mathrm{x}$ QuantiTect SYBR Green PCR Master Mix , $1 \mu 1$ 10x Forward Primer, $1 \mu$ 10x Reverse Primer, $0.3 \mu 1$ $\mathrm{MgCl}_{2}$ and RNase free water to a total volume of $10 \mu \mathrm{l}$. The PCR program was as follows: $50^{\circ} \mathrm{C}$ for $2 \mathrm{~min}$, initial activation step $95^{\circ} \mathrm{C}$ for $15 \mathrm{~min}$ followed by 40 cycles of $95^{\circ} \mathrm{C}$ for $15 \mathrm{sec}$ (denaturation) $\mathrm{x}^{\circ} \mathrm{C}$ for $30 \mathrm{sec}(\mathrm{x}=$ annealing temperature depending on the $\mathrm{Tm}$ of the used primers) and $72^{\circ} \mathrm{C}$ for $30 \mathrm{sec}$ (extension).

Primer sequences are provided in the oligos section. Sdha was used as endogenous reference. For standard curves, samples of undifferentiated or differentiated ESCs and maGSCs were used. Selection of the appropriate sample for the standard curve was based on preliminary experiments testing detection of expression of each gene by RT PCR in the sample with the respective annealing temperature and for 35 cycles. As in miRNAs, specificity of the PCR products was confirmed with subsequent cloning and sequencing while reliability of Real-time PCR data was also assessed in connection with the respective dissociation curves.

\subsubsection{Gel electrophoresis}

Gel electrophoresis is the technique by which a mixture of charged macromolecules, especially nucleic acids and proteins are separated in an electrical field according to their mobility which is directly proportional to macromolecule's charge to mass ratio. 


\section{$\underline{\text { PAGE electrophoresis }}$}

PAGE electrophoresis was used at the beginning to separate the products of miRNAs qPCRs, since these were expected to be $<50 \mathrm{bp}$ and this method provides a better separation in these sizes. However, it was found that due to the universal adaptor added by the kit, products were $>70 \mathrm{bp}$ and thus in the following experiments regular agarose gel electrophoresis was applied.

\section{Agarose gel electrophoresis}

Agarose gels are used to electrophorese nucleic acid molecules from as small as 50 bases to more than 50 kilobases, depending on the concentration of the agarose and the precise nature of the applied electrical field (constant or pulse). Usually, $1 \mathrm{~g}$ of agarose was added in $100 \mathrm{ml} 0.5 \mathrm{x}$ TBE buffer, boiled in the microwave to dissolve the agarose, then cooled down to about $60^{\circ} \mathrm{C}$ before adding $3 \mu$ lethidium bromide $(10 \mathrm{mg} / \mathrm{ml})$. This $1 \%$ agarose gel was poured onto a horizontal gel chamber. For running of products of miRNA Real-time PCR for subsequent cloning, 3,5\% agarose gels were used.

\subsection{Methods for subcloning and sequencing of RT- PCR products}

\subsubsection{Gel extraction}

QIAquick gel extraction method: This method is designed to extract and purify DNA from agarose gels. Up to $400 \mathrm{mg}$ agarose can be processed per spin column. The principle of this method depends on 
selective binding of DNA to uniquely designed silica gel membranes. Excised DNA fragments in agarose were isolated as described in QIAquick Spin Handbook supplied by the producer (Qiagen). In brief, to the excised DNA fragment from agarose gel, 3 volumes of QG buffer were added and then incubated at $50^{\circ} \mathrm{C}$ for $10 \mathrm{~min}$. After the gel slice was dissolved completely, the solution was applied over a QIAquick column and centrifuged. The flow through was discarded and the column was washed with PE buffer. After drying the column, it was placed into a fresh microcentrifuge tube. To elute DNA, $50 \mu \mathrm{l}$ of EB buffer was applied to the centre of the QIAquick membrane and centrifuged.

\subsubsection{Ligation}

Taq and other polymerases have a terminal transferase activity that results in the nontemplate addition of a single nucleotide to the $3^{\prime}$ ends of PCR products. In the presence of all 4 dNTPs, dATP is preferentially added. This terminal transferase activity is the basis of the TA - cloning strategy. For cloning of these PCR products, the pGEM®T-easy Vector system that has 5'dT overhangs was used. The following constituents were mixed:

$50 \mathrm{ng}$ of pGEM-T Easy vector

PCR product (1:3 vector to insert ratio)

$1 \mu \mathrm{l} 4$ DNA Ligase 10x buffer

$1 \mu \mathrm{T} 4$ DNA Ligase

in a total volume of $10 \mu \mathrm{l}$. The content was mixed by pipetting and the reaction was incubated at $\mathrm{RT}$ for $3 \mathrm{hrs}$ for inserts originating from mRNAs, and at $4 \mathrm{C}$ overnight for inserts originating from miRNAs. 


\subsubsection{Transformation of competent bacteria}

Transformation of the bacteria was done by gently mixing one aliquot of competent bacteria $(50 \mu \mathrm{l})$ with $10 \mu \mathrm{l}$ of ligation reaction. After incubation for $30 \mathrm{~min}$ on ice, bacteria were heat shocked for $45 \mathrm{sec}$ at $42^{\circ} \mathrm{C}$ and cooled down for $5 \mathrm{~min}$ on ice. After adding $900 \mu \mathrm{l}$ of S.O.C. medium, bacteria were incubated at $37^{\circ} \mathrm{C}$ with shaking for $1 \mathrm{hr}$ to allow recovery of heat shocked bacteria. They were then plated out on LB-agar plates containing ampicillin $(50 \mu \mathrm{g} / \mathrm{ml})$ and whenever required $1 \mathrm{mM}$ IPTG and X-Gal $40 \mathrm{mg} / \mathrm{ml}$ were added for "BlueWhite" selection.

\subsubsection{Cloning}

A single Escherichia coli colony was inoculated in $5 \mathrm{ml}$ of LB medium with ampicillin and incubated in a shaker for $16 \mathrm{hrs}$ at $37^{\circ} \mathrm{C}$ with a speed of $160 \mathrm{rpm}$.

\subsubsection{Small-scale isolation of plasmid DNA (Mini preparation)}

One $\mathrm{ml}$ of the above mentioned saturated culture was used for making glycerol stocks and rest of the culture was centrifuged at 5,000xg for 15 min. Bacterial glycerol stocks were made suspending $500 \mu \mathrm{l}$ bacteria in $300 \mu 180 \%(\mathrm{v} / \mathrm{v})$ sterile glycerol. The suspension was mixed well and stored at $-80^{\circ} \mathrm{C}$. The pellet was resuspended in $100 \mu \mathrm{l}$ of solution P1. The bacterial cells were lysed with $200 \mu$ of P2 solution and then neutralized with $150 \mu \mathrm{l}$ of solution P3. The precipitated solution was incubated on ice for $15 \mathrm{~min}$ and centrifuged 
at $13,000 \mathrm{xg}$ at $4{ }^{\circ} \mathrm{C}$. The supernatant was transferred into a new tube and centrifuged again for $10 \mathrm{~min}$ at $13,000 \mathrm{xg}$. To the supernatant $1 \mathrm{ml}$ of $100 \%$ ethanol was added to precipitate the DNA. It was then stored on ice for $15 \mathrm{~min}$, centrifuged at full speed for $20 \mathrm{~min}$, and finally the pellet was washed with $70 \%$ ethanol and after air dried it was dissolved in $30 \mu \mathrm{l}$ of TE buffer.

\subsubsection{Digestion}

Successful ligation and cloning was confirmed through digestion of the plasmid DNA with the restriction enzyme recommended from pGEM®T-easy manufacturer, the EcoRI. Restriction enzymes are class of bacterial enzymes that cut DNA at specific sites. Restriction enzyme digestion was performed by incubating the plasmid DNA with the appropriate amount of restriction enzyme in its respective buffer as recommended by the supplier, and at $37^{\circ} \mathrm{C}$ for $90 \mathrm{~min}$.

\subsubsection{Cleaning of DNA sample}

For successful downstream applications, isolated plasmid DNA should be of high purity. To clean the plasmid DNA from undesired byproducts of previous isolation processes, the Montage PCR Kit was used, following the instructions recommended by the supplier.

\subsubsection{Sequencing}

The non-radioactive sequencing was achieved with Dye Terminator Cycle Sequencing-Kit. The principle of this is based on the Sanger method. The sequencing reaction was carried out in a total volume of 
$10 \mu$ containing $1 \mu \mathrm{g}$ plasmid DNA or $100-200$ ng purified PCR products, 5 pmol primer and $3 \mu$ reaction mix (contains dNTPs, dideoxy dye terminators and Taq DNA polymerase). Elongation and chain termination take place during the following program in a thermocycler: $5 \mathrm{~min}$ denaturing followed by 25 cycles: $95^{\circ} \mathrm{C}, 30 \mathrm{sec}$ (denaturing); $55^{\circ} \mathrm{C}, 30 \mathrm{sec}$ (annealing); $60^{\circ} \mathrm{C}, 2 \mathrm{~min}$ (elongation). After the sequencing reaction, the reaction products were analyzed with an automatic sequencing equipment, namely MEGA BACE 1000 .

\subsection{Protein expression analysis methods}

\subsubsection{Protein extraction}

Proteins were extracted from $\sim 30 \mathrm{mg}$ frozen mouse tissues (disrupted before by mortar and pestle) or from cells in pellets $\left(10^{5}-5 \times 10^{6}\right.$ cells were previously trypsinized and pellets were washed with PBS) by homogenization in protein lysis buffer. Lysates were maintained on ice for $60 \mathrm{~min}$ and then sonicated on ice and centrifuged at 12,000xg for $10 \mathrm{~min}$ at $4^{\circ} \mathrm{C}$. Supernatant was collected, quantified and stored at $-80^{\circ} \mathrm{C}$ or used immediately for Western blotting.

\subsubsection{Determination of protein concentration}

To determine the protein concentration, Bio-Rad protein assay was employed, which is a dye-binding assay based on the differential colour change of a dye in response to various concentrations of protein. The assay is based on the observation that the absorbance maximum for an acidic solution of Coomassie Blue G-250 shifts from 
494 to $595 \mathrm{~nm}$ when the binding to protein occurs. The bovine serum albumin (BSA) stock solution of $1 \mathrm{mg} / \mathrm{ml}$ was diluted in order to obtain standard dilutions in range of $10 \mu \mathrm{g} / \mathrm{ml}$ to $100 \mu \mathrm{g} / \mathrm{ml}$. The BioRad's color reagent was diluted 1:5 with $\mathrm{H}_{2} \mathrm{O}$ and filtered through $0.45 \mu \mathrm{m}$ filters. In a 96-well microtiter plate, $20 \mu \mathrm{l}$ of each standard dilution and the samples to be measured were pipetted with $280 \mu \mathrm{l}$ of the colour reagent. The absorption of the colour reaction was measured at $595 \mathrm{~nm}$ in a microplate reader (Microplate Reader 450, Bio-Rad).

\subsubsection{SDS-PAGE for the separation of proteins}

The NuPAGE Pre-Cast Gel System (Invitrogen) is a polyacrylamide gel system for high performance gel electrophoresis and is based on SDS-PAGE gel chemistry. It consists of NuPAGE Bis-Tris Pre-Cast Gels and specially optimized buffers which have an operating $\mathrm{pH}$ of 7.0. The NuPAGE System is based upon a Bis-Tris-HCl buffered $(\mathrm{pH}$ 6.4) polyacrylamide gel, with a separating gel that operates at $\mathrm{pH}$ 7.0. While NuPAGE Bis-Tris Gels do not contain SDS, they are formulated for denaturing gel electrophoresis applications only.

7,5 $\mu$ of NuPAGE LDS Sample Preparation Buffer 4x ( $\mathrm{pH} 8.4)$ were mixed with $2 \mu \mathrm{DTT} 0,1 \mathrm{M}, 20 \mu \mathrm{g}$ of whole protein extract and water to a total volume of $30 \mu \mathrm{l}$. The samples were denaturated by heating in $70^{\circ} \mathrm{C}$ for $10 \mathrm{~min}$ and cooled at ice for $5 \mathrm{~min}$. Samples were then loaded in NuPAGE Bis-Tris Gels of acrylamide concentration of $10 \%$ and run in $1 \mathrm{x}$ NuPAGE MOPS Buffer at $60 \mathrm{~V}$ for about 3,5 hrs at RT producing an expected separation range. To determine the molecular weight of the proteins on the gel, $10 \mu \mathrm{l}$ of a pre-stained molecular weight standard (See Blue Plus2, Invitrogen) was also loaded. 


\subsubsection{Western blotting}

After the electrophoresis of proteins the gel and the Hybond-C Extra membrane, which was cut at the size of the gel, were first moistened in transfer buffer. 10 pieces of GB004 Whatman filter paper were also cut at the size of the gel. First, five papers soaked with transfer buffer were placed on blotting machine's lower plate and then membrane was placed over them. Next the gel was placed avoiding any air bubbles. Another 5 Whatman papers soaked with transfer buffer were placed over to complete the sandwich model. The upper plate was placed over this sandwich and the transfer was carried out at $150 \mathrm{~mA}$ for $15 \mathrm{~min}$ and $300 \mathrm{~mA}$ for $60 \mathrm{~min}$. Membrane was then used for incubation with antibodies. To confirm transfer efficiency of proteins onto the membranes, the gel was incubated for $30 \mathrm{~min}$ in Coomassie blue solution at RT.

The membrane was first incubated in blocking buffer I (B1) with 5\% non-fat dry milk for $1 \mathrm{hr}$ at RT in order to block unspecific binding sites, followed by incubation with $2 \%$ milk in B1 buffer for 5 min. Membrane was then incubated with a primary antibody at the recommended antibody dilution in buffer B1 with $2 \%$ non-fat dry milk for overnight at $4{ }^{\circ} \mathrm{C}$. Then, the membrane was washed 3 times in $\mathrm{B} 1$ buffer with $2 \%$ dry milk for $60 \mathrm{~min}$ and then incubated with the HRP conjugated secondary antibody in B1 buffer with $2 \%$ non-fat dry milk for $1 \mathrm{hr}$ at $4^{\circ} \mathrm{C}$. After this step, the membrane was washed 3 times in B1 buffer with $2 \%$ dry milk, one time in PBS for 5 min at $4^{\circ} \mathrm{C}$. Finally, protein bands were visualized using enhanced chemiluminescence as described by the manufacturer (Santa Cruz Biotech.). When the expected band size allowed it, membranes were reused for one more time and were incubated with another primary antibody after blocking. The following antibodies were used: $\alpha-$ Tubulin (dil 1:5,000), Oct-4 (dil 1:500), Sox-2 (dil 1:1,000), and the respective anti-rabbit or anti-mouse IgG-Peroxidase antibodies. 


\subsubsection{Immunocytochemistry}

First, the culture media was removed from the cells in microscopical chambers and cells were rinsed with PBS. After that $1 \mathrm{ml} \mathrm{4 \%} \mathrm{PFA}$ (made fresh) per chamber was added and incubated for $10 \mathrm{~min}$ at RT for fixing the cells. Then, the cells were washed 3 times with PBS and permeabilized by adding $1 \mathrm{ml}$ blocking buffer II / plate containing Triton X-100. The permeabilization was carried out for $10 \mathrm{~min}$ at RT. After washing, the cells were blocked in blocking buffer II and incubated with the respective diluted primary antibody for $1 \mathrm{hr}$ to overnight at $4{ }^{\circ} \mathrm{C}$. Then cells were washed again and stained with the secondary antibody diluted in the same buffer applied for primary for $1 \mathrm{hr}$ at RT. For immunofluorescence staining of SSEA-1, the antibody contained in the ES Cell Characterization Kit (Chemicon) was used. An anti-rabbit IgG Cy3-conjugated antibody (Sigma-Aldrich) was used as secondary antibody. Slides were viewed in a BX60 fluorescence microscope (Olympus). Before microscopical analysis the cells were rinsed 2 times with PBS and mounted with DAPI (Vector).

\subsection{Bioinformatics methods and software}

\subsubsection{Statistical analysis of data}

For statistical analysis of data, the STATISTICA software was used. Data are expressed as the mean $\pm \mathrm{SD}$. A one-way analysis of variance (ANOVA) followed by Fischer LSD's multiple comparison tests was used for statistical analysis with $\mathrm{P}$ less than 0.05 considered statistically significant. 


\subsection{2 miRNA target prediction}

For prediction of miRNA targeting sites in mRNAs' 3 'UTRs the TARGETSCAN Software was used (www.targetscan.org).

\subsubsection{Sanger miRNA database (miRBase)}

All miRNA sequences were provided by or correlated with the sequences stored in the Sanger miRNA data base (http://microrna.sanger.ac.uk).

\subsubsection{Blast}

The obtained DNA sequences were analyzed with a BLAST program by using the sequence data base Genbank (www.ncbi.nlm.nih.gov) and EMBL (www.ebi.ac.uk).

\subsubsection{Western blotting density determination software}

Levels of Oct-4 and Sox-2 from Western blots of two independent experiments were quantified densitometrically with QuantityOne software (Bio-Rad) and normalized to $\alpha$-Tubulin.

\subsubsection{SDS software}

Visualization and primary raw data processing of the qPCR results was performed by the SDS Software. 


\subsubsection{Microsoft Excel}

Processing of the qPCR raw data and visualization of the results was done using teh Microsoft Office Excel Software. 


\section{Results}

\section{1 microRNA array expression profiling of maGSCs compared with ESCs}

In order to address the question whether ESCs and maGSCs are distinct or equivalent cell types from the viewpoint of miRNA expression patterns, the global miRNA expression profiles of maGSCs and ESCs were examined by miRNA arrays.

miRNA array analysis was carried out for two maGSC lines, maGSC 129SV and maGSC Stra8, and their respective ESC lines, ESC R1 and ESC Stra8. Every hybridization was performed in duplicates (two identical slides for each comparison/hybridization), and every miRNA was present four times on each slide. The experiment layout was designed as follows (each arrow depicts one hybridization, as described in Materials and Methods):

Experiment A:

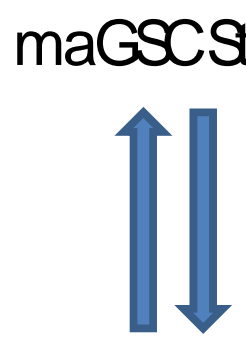

maGsC129SV

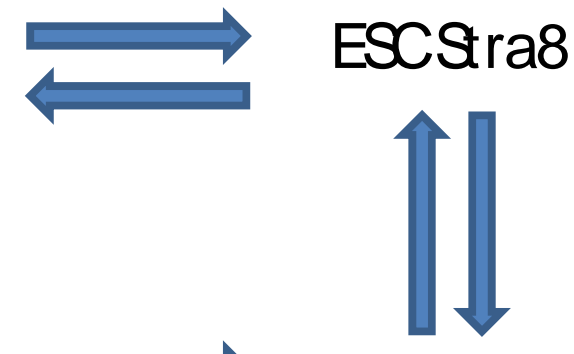

ESCR1 
This layout allows the simultaneous comparison of each cell line with all others ensuring at the same time the statistical validity of the results (four technical replicates).

In case of maGSC 129SV and ESC R1 the experiment was carried out one more time (Experiment B), with the same number of technical replicates, as follows:

Experiment B:

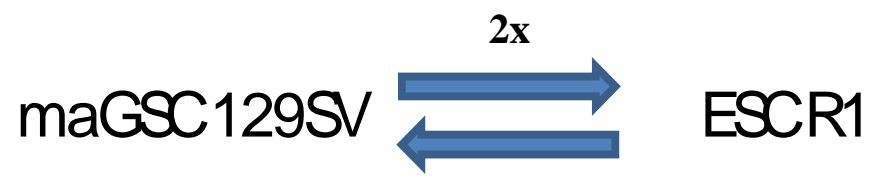

In this case maGSC 129SV and ESC R1 were cultured independently from cells of Experiment A to allow their use as a biological replicate.

miRNA arrays tested included more than 1700 capture probes, covering all human, mouse and rat microRNAs annotated in miRBase 11.0, as well as all viral microRNAs, related to these species. The coverage of this array to the latest miRBase version (miRBase 12.0) is $98 \%, 97 \%$ and $100 \%$ for human, mouse and rat, respectively (http://microrna.sanger.ac.uk). In this study only mouse miRNAs were of interest. More information about the array contents and layout are mentioned in Materials and Methods section 2.4.2. The array results are presented in Tables 1, 2 and 3 and in Appendix 6.

maGSC Stra 8 cells were found to differ from all other cell lines tested concerning miRNA array expression profiling. In detail, five out of nine differentially expressed miRNAs between ESC Stra8 and maGSC Stra8 differ also in the comparisons of maGSC Stra8 with maGSC $129 \mathrm{SV}$ and ESC R1. These miRNAs (marked with orange highlighting/Table 1) are the following: miR-762, miR-494, miR-720, miR-711 and miR-689. Four others (miR-184, miR-712*, miR-21 and 
miR-301a) were found to differ only between maGSC Stra8 and the respective ESC line (Table 1).

\begin{tabular}{|c|}
\hline ESCStra8 < \\
\hline miR-762 \\
\hline miR-494 \\
\hline miR-720 \\
\hline miR-711 \\
\hline miR-689 \\
\hline miR-184 \\
\hline miR-712 \\
\hline miR-21 \\
\hline miR-301a \\
\hline ESCStra8 $>$ \\
\hline \\
\hline \\
\hline
\end{tabular}

Table 1. Differentially expressed miRNAs between ESC Stra8 and the respective maGSCs (maGSC Stra8) $<$ and $>$ depict lower or higher expression levels respectively of these miRNAs in ESC Stra8 compared with maGSC Stra8. $\mathrm{x}$-fold changes and statistical values are presented in Appendix 6 .

In contrast, all the differences observed between ESC R1 and maGSC 129SV were specific only for this comparison (Table 2/Exp A). When the experiment was repeated (Table 2/Exp B), most of these differences were eliminated. In detail, most of the miRNAs that were found to be expressed in higher levels in ESC R1 belong to miR-17-92 cluster (miR-17, mir-19b and miR-20a) or its paralogue miR-106a363 cluster (miR-106a and miR-20b). In addition, differential expression was found for members of miR-467 cluster and miR-803, as well as for miR-22 and miR-689 that were upregulated in maGSCs. 


\begin{tabular}{|c||c|}
\hline Experiment A & Experiment B \\
\hline \hline ESCR1< & ESCR1< \\
\hline miR-22 & \\
\hline miR-689 & \\
\hline ESCR1 $>$ & \\
\hline miR-20a & ESCR1> \\
\hline miR-17 & \\
\hline miR-19b & \\
\hline 20b & miR-19b \\
\hline miR-467b & miR-20b \\
\hline miR-467a*/467d* & \\
\hline miR-106a & \\
\hline miR-803 & \\
\hline & \\
\hline & \\
\hline
\end{tabular}

Table 2. Differentially expressed miRNAs between ESC R1 and the respective maGSCs (maGSC 129SV) in either experiment A or B. miRNAs differentially expressed in both experiments are marked with red. $<$ and $>$ depict lower or higher expression levels respectively of these miRNAs in ESC R1 compared with maGSC 129SV. x-fold changes and statistical values are presented in Appendix 6.

Finally, most differences between the two maGSC lines belong either to these that are common between maGSC Stra8 and other cell lines mentioned above (marked with orange highlighting/Table 3), or could be genetic background related, since they are observed also between ESC Stra8 and ESC R1 (marked with green highlighting/Table 3). miRNAs that differed between the different maGSC lines but do not belong to the above mentioned groups (orange/green) consist of miR335_5p, miR-22, miR-294 and miR-142_3p. 


\begin{tabular}{|c|}
\hline maGSCSta8 $<$ \\
\hline miR-335_5p \\
\hline miR-124 \\
\hline miR-182 \\
\hline miR-96 \\
\hline miR-183 \\
\hline miR-292_3p \\
\hline miR-295 \\
\hline miR-22 \\
\hline miR-294 \\
\hline maGSCStra8 > \\
\hline miR-762 \\
\hline miR-494 \\
\hline miR-720 \\
\hline miR-711 \\
\hline miR-689 \\
\hline miR-142_3p \\
\hline miR-706 \\
\hline miR-142_5p \\
\hline
\end{tabular}

Table 3. Differentially expressed miRNAs between maGSC Stra8 and maGSC 129SV. < and > depict lower or higher expression levels respectively of these miRNAs in maGSC Stra8 compared with maGSC 129SV. $x$-fold changes and statistical values are presented in Appendix 6.

Detailed array results including $\mathrm{P}$ values and $\mathrm{x}$-fold changes in miRNA expression are available in Appendix 6. 


\subsection{Validation of the array results in maGSCs from $129 / \mathrm{Sv}$ and other mouse strains}

Since aim of this study was to shape the similarities and differences between ESCs and maGSCs, validation of the array results concentrated only in the miRNAs that differed between ESCs and maGSCs. In addition, since all other maGSC strains were derived similarly to maGSC 129SV, miRNAs differentially expressed in maGSC Stra8 were excluded from the validation procedure since derivation method of these cells (genetic selection for Stra8) seems to affect their gene expression signature.

Most of miRNAs that differed between maGSC 129SV and ESC R1 in Experiment A belonged to miR-17-92 cluster or its paralogue miR106a-363. Differences in two miRNAs from these families (miR-20b and miR-19b) persisted when the experiment was repeated (Exp B) (red highlighting/Table 2).

qPCR was performed only for miR-20b and miR-19b that were found to differ in both experiments between ESC R1 and maGSC 129SV and confirmed the array results (Fig. 3).

In addition maGSCs from mouse strains C57Bl and FVB were compared with their respective ESCs to find whether the differences in these two miRNAs are strain related or not. Figure 3 shows that differences observed in these miRNAs are restricted to $129 / \mathrm{Sv}$ background. In other strains, miRNA levels between maGSCs and ESCs were similar for these two miRNAs. 


\section{miR-19b}

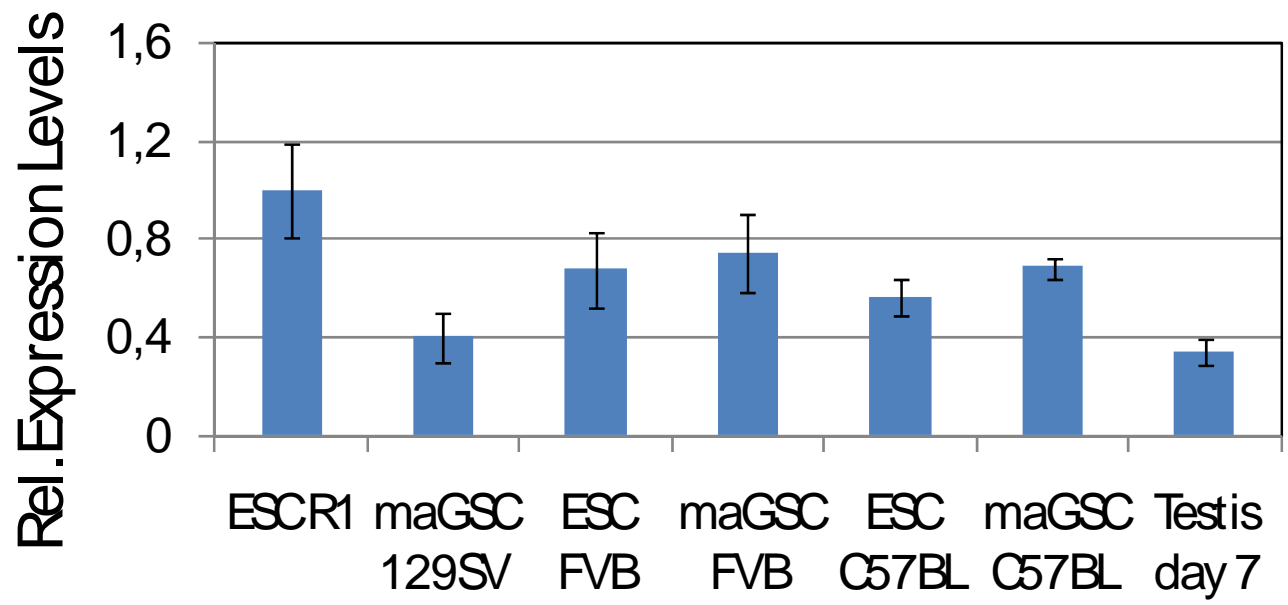

miR-20b

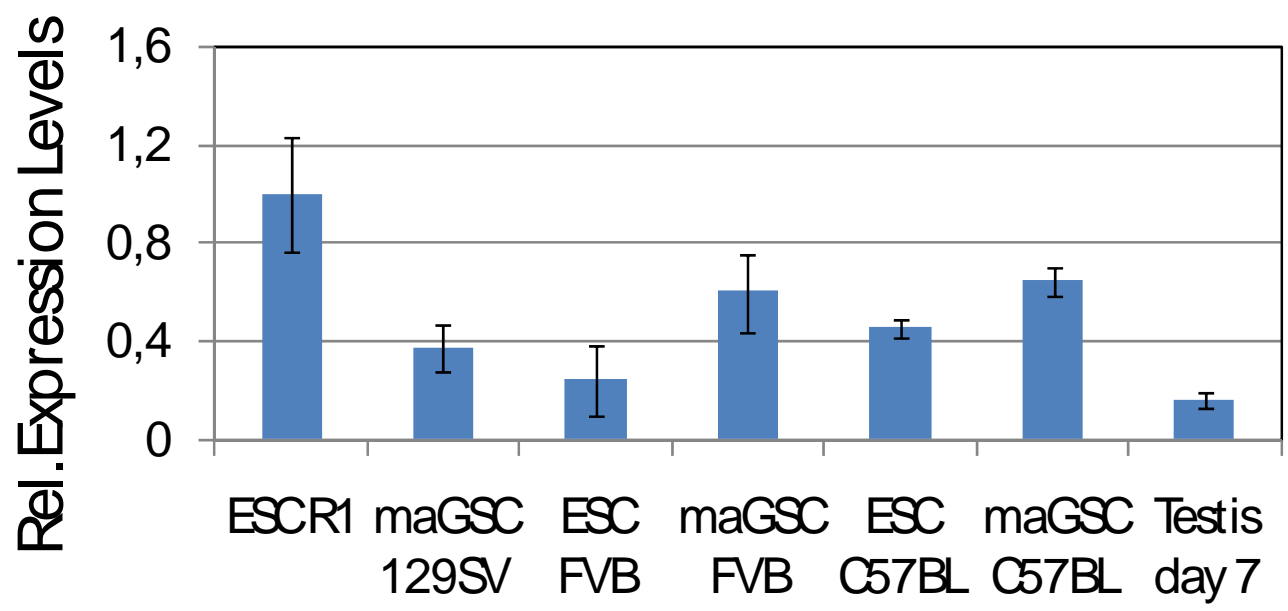

Figure 3. Expression levels of miR-19b and miR-20b detected by Real-time PCR in ESCs and maGSCs from different mouse strains. Expression levels are normalized to the expression of the endogenous control (RNU6B). ESC R1 was used as calibrator (cal). 


\subsection{Expression of $\mathrm{miR}-290$ and $\mathrm{miR}-302$ miRNA clusters in maGSCs and ECCs}

Expression of ESC-specific miRNA clusters 290 and 302 was tested in maGSCs derived from the following mouse strains: $129 / \mathrm{Sv}$ (maGSC 129SV), C57BL/6 (maGSC C57BL), FVB (maGSC FVB) and from the transgenic line Stra8-EGFP/ROSA26 (maGSC Stra8). All maGSC lines were cultured under standard ESC culture conditions. In addition, the expression of ESC specific miRNAs was studied in the teratocarcinoma cell line F9 (ECCs) which was found to share many similarities with pluripotent cells (Andrews, 2002). Finally, miRNA expression was tested in adult mouse testis.

MEFs and NIH/3T3 cells served as negative controls, while ESCs derived from mouse strains $129 / \mathrm{Sv}$ (ESC R1) and Stra8EGFP/ROSA26 (ESC Stra8) were used as positive controls.

To exclude the possibility that differences found in miRNA levels between different cell lines could be attributed to spontaneous differentiation of the cells due to improper culture conditions, pluripotent state was evaluated for all cell lines and tissues tested. Expression levels of Oct-4 and Sox-2 proteins, which are the most widely used markers for pluripotency, were evaluated with Western blotting.

Both Oct-4 and Sox-2 were expressed in all maGSC, ESC and ECC lines tested (Fig. 4). Expression of Oct-4 was not detected in testis by Western blotting. 


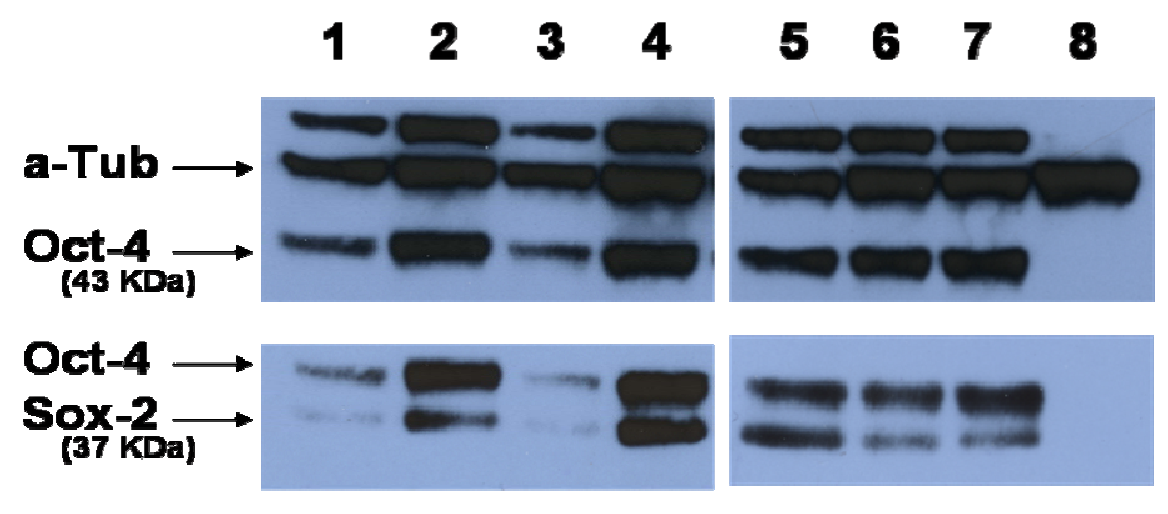

1. ESCR1

2. macsc $1295 V$

3. ESC Stra8

4. macsc Stra8

5. maGSC FVB

6. macsc C57BL

7. $F 9$

8. testis

Figure 4. Expression of Oct-4 and Sox-2 proteins by Western blotting. The same membrane was used for both antibodies. a-Tubulin served as loading control.

After the pluripotent state of the cells was confirmed, expression of the following members of miR-290 and miR-302 miRNA clusters was tested with quantitative reverse transcription PCR:

- miR-290 family: miR-290_5p, miR-291_3p, miR-292_3p, miR-293, miR-294 and miR-295

- miR-302 family: miR-302a, miR-302b and miR-302d

This set of ESC-specific miRNAs was found to be expressed not only in ESCs but also in all maGSC lines tested as well as in F9 cells. No expression was detected in MEFs, NIH/3T3 cells and mouse testis (Fig. 5).

Interestingly, miRNA expression pattern in maGSC Stra8 differed from maGSCs derived from other mouse strains by demonstrating lower and higher levels of miR-290 and miR-302 family, respectively. In addition, differences were observed between maGSC 129SV and 
ESCs from the same mouse strain (ESC R1). In this case ESC R1 and maGSC 129SV were from different passage numbers.

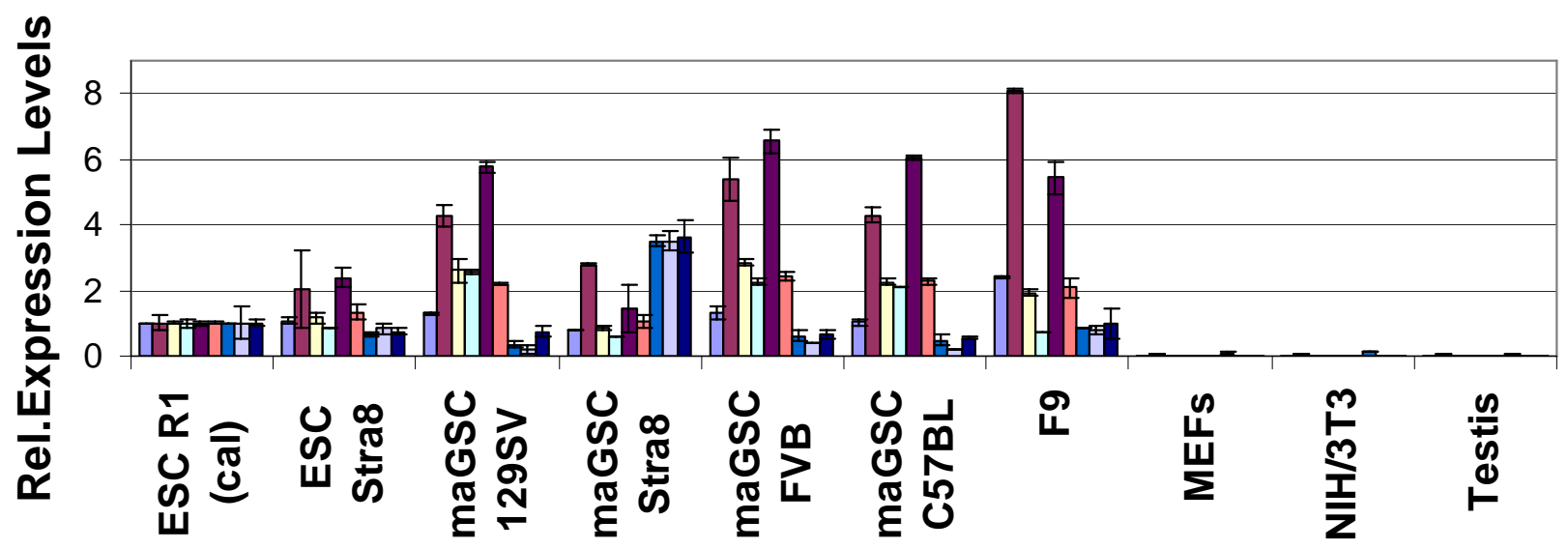

Figure 5. Expression levels of ESC-specific miRNAs detected by Real-time PCR. Expression levels are normalized to the expression of the endogenous control (RNU6B). ESC R1 (of a different passage number of that used in all other experiments) was used as calibrator (cal). 


\subsection{Expression of $\mathrm{miR}-290$ and $\mathrm{miR}-302$ miRNA clusters in ESCs and maGSCs after culture for many passages and upon loss of pluripotency}

To exclude expression of these miRNAs only in maGSCs of early passage numbers, expression was tested also after culture for 10 passages. To confirm that expression of these miRNAs is restricted only to undifferentiated maGSCs, lack of expression was tested in differentiated maGSCs after deprivation of LIF and induction with RA for 35 days (Gel+RA 35 days). maGSCs from the mouse strain 129/Sv were used. The respective ESC line from the same mouse strain (ESC R1) and of the same passage number was used as control.

Pluripotent state of the cells was evaluated as reported above (Fig. 6). In addition, expression of SSEA-1, which is also a well known pluripotency marker, was tested by immunofluorescence (Fig. 7). Under standard ESC culture conditions maGSCs from the 129/Sv mouse strain express Oct-4 and Sox-2 at levels similar to those of the respective ESCs cultured for the same period of time and number of passages. In contrast, no expression of pluripotency markers was detected in differentiated ESCs and maGSCs, confirming that exposure for a long time to differentiation conditions results in loss of pluripotency in both cell types. 

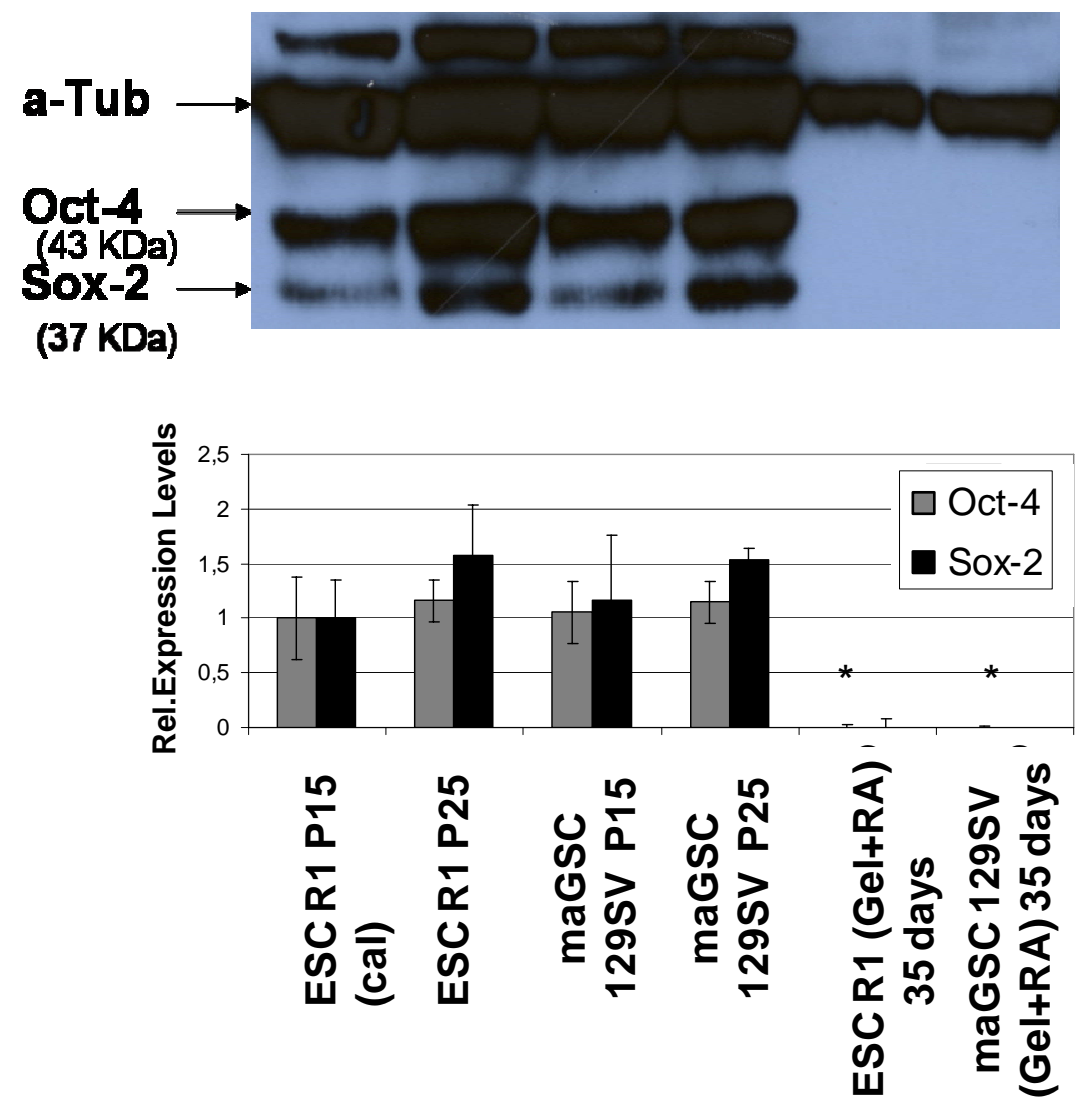
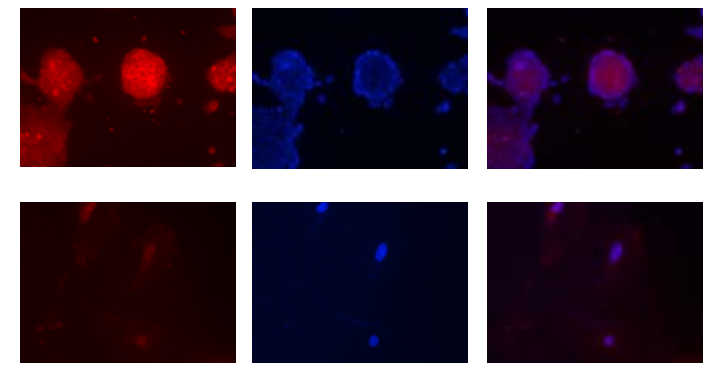

Gel+RA 35

Days

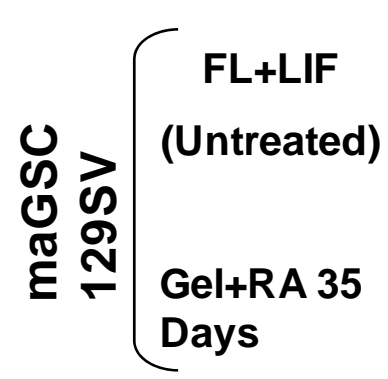

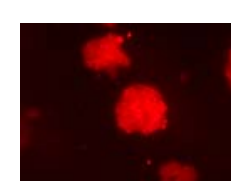

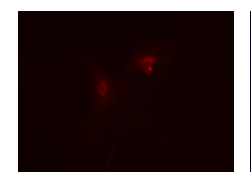

Cy-3
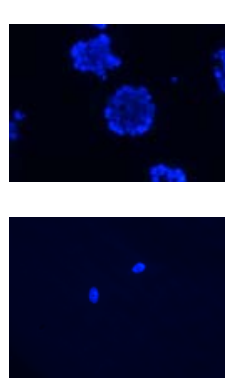

DAPI Overlay

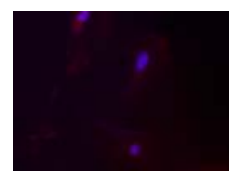

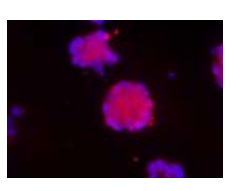

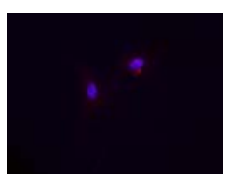

Figure 6. Expression of pluripotency markers in ESCs and maGSCs from $129 / \mathrm{Sv}$ background after culture for 35 days under standard ESC culture conditions (from passage 15 to passage 25 ) or in Gel+RA $(0,1 \%$ gelatin coated flasks without LIF but with RA). Levels of Oct-4 and Sox-2 proteins obtained by Western blotting were quantified densitometrically and normalized to levels of endogenous control (a-tub). Asterisks indicate statistical significance in comparison with untreated cells. $\mathrm{Cal}=$ calibrator.

Figure 7. Immunostaining for SSEA-1 (Cy-3) in ESCs and maGSCs from 129/Sv background under standard ESC culture conditions (untreated) and after culture for 35 days in Gel+RA $(0,1 \%$ gelatin coated flasks without LIF but with RA). DAPI was used for staining of the nuclei. 
Like ESCs, after culture for 10 passages under standard ESC culture conditions, maGSCs retain expression of the ESC-specific miRNAs (Fig. 8). However, these levels seem to vary between different passage numbers and independently cultured cells from the same cell line as shown in Appendix 3 and mentioned below (section 3.4.3.).

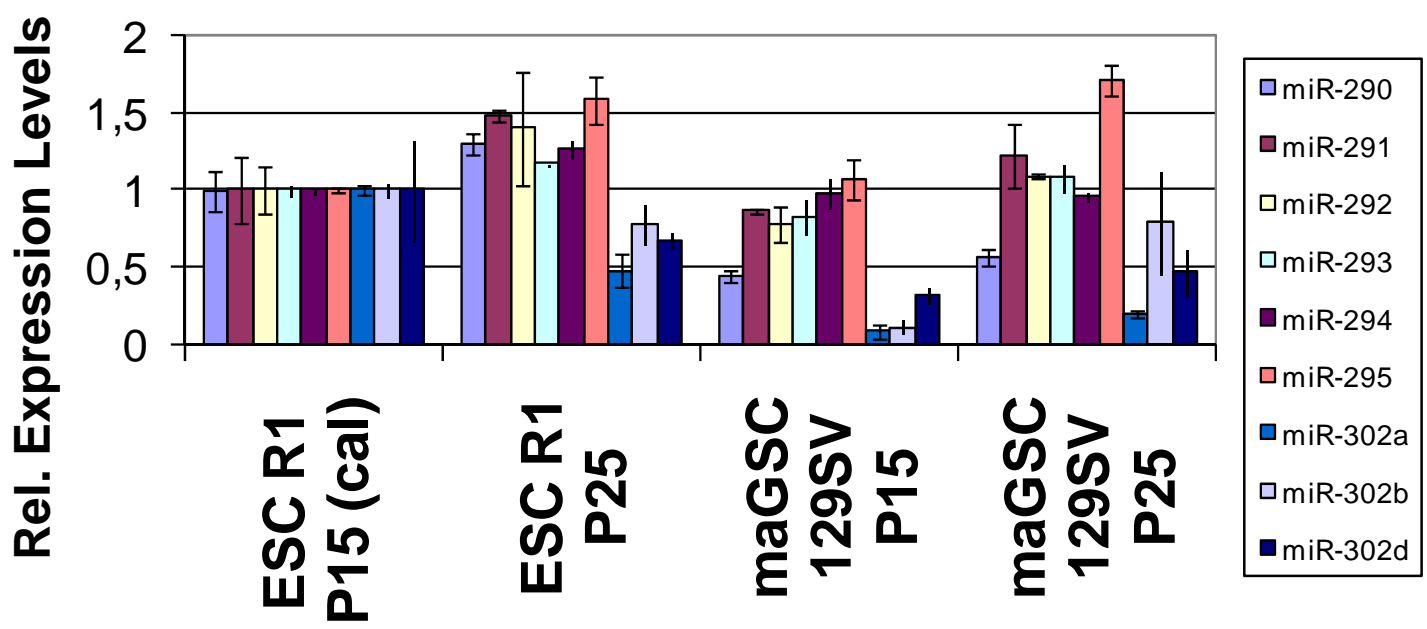

Figure 8. miRNA expression levels in ESC R1 and maGSC 129SV of passages 15 and $25 . \mathrm{Cal}=$ calibrator.

In addition, like in differentiated ESCs, loss of pluripotency in maGSCs resulted in hardly detectable expression levels of ESCspecific miRNAs (Fig. 9). 


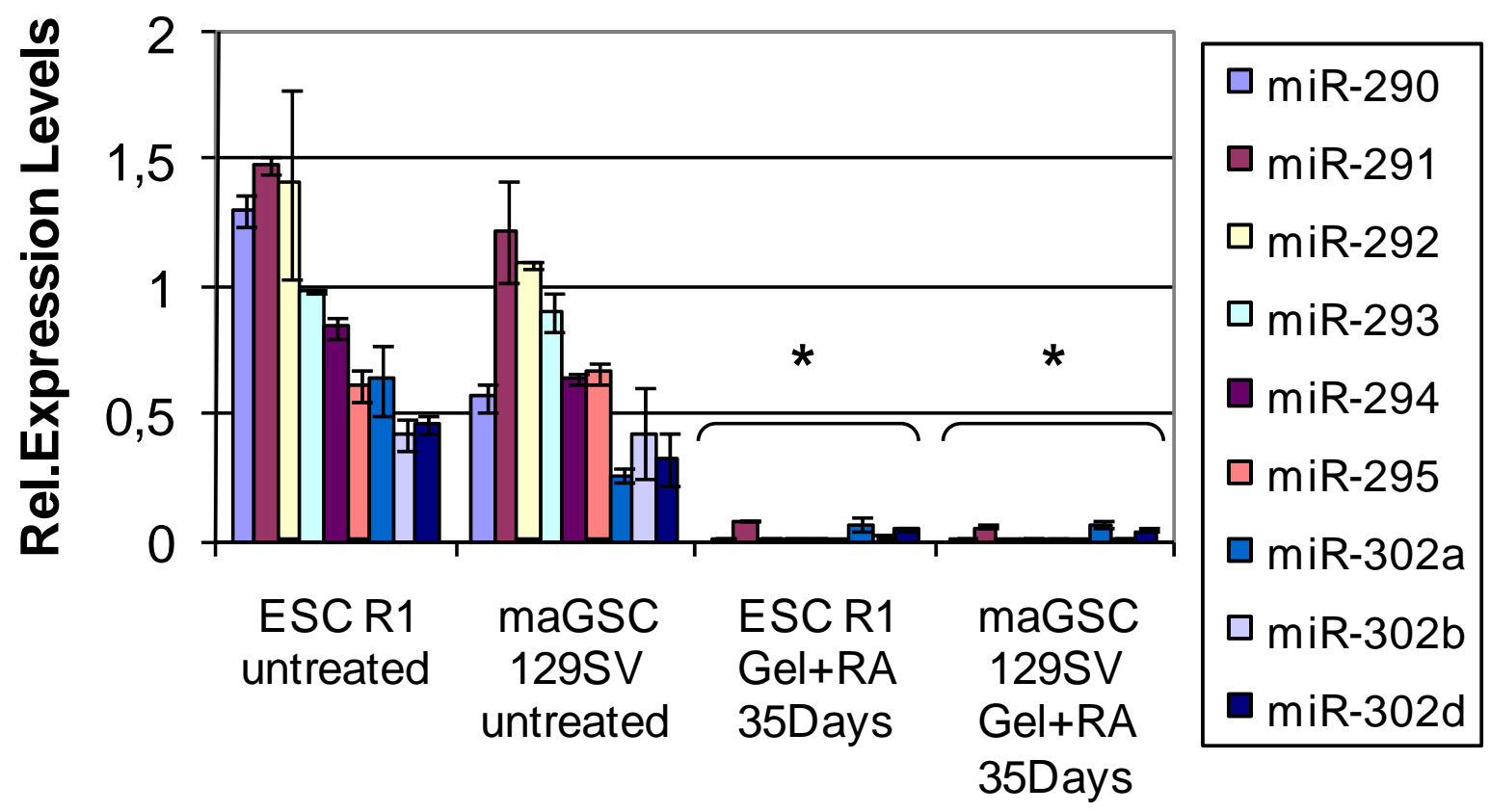

Figure 9. miRNA expression levels in untreated ESC R1 and maGSC 129SV cells compared with the respective cells cultured for 35 days in Gel+RA (gelatin coated flasks without LIF but with RA). Asterisks indicate statistical significance. 


\subsection{Expression profiles of $\mathrm{miR}-290$ and $\mathrm{miR}-302$ miRNA clusters during in vitro differentiation of pluripotent cells}

\subsubsection{Differentiation strategy}

To induce differentiation of pluripotent cells in vitro, a number of factors are commonly used. Among them deprivation of LIF and addition of retinoic acid (RA) has been shown to be crucial for allowing and inducing differentiation. The effects of these factors on the expression of ESC-specific miRNA families 290 and 302 were examined in maGSCs in comparison to ESCs.

Figure 10 summarizes the strategy that was followed. ESCs and maGSCs of passage P16 from the mouse strain 129/Sv (ESC R1 and maGSC 129SV, respectively) were cultivated for 5, 10 and 21 days under different culture conditions:

- Feeder layer (FL), LIF and retinoic acid (FL+LIF+RA)

- $0.1 \%$ gelatin coated flasks $\underline{(\mathbf{G e l})}$

- $0.1 \%$ gelatin coated flasks and retinoic acid (Gel+RA) or (Gel+RA from day 0)

- Cells that were cultivated for 5 days in $0.1 \%$ gelatin coated flasks and then induced by retinoic acid for 5 days (Gel+RA from day 5)

Cells were collected at day 5,10 and 21 for further testing.

To assess the degree of differentiation and loss of pluripotency of treated cells, levels of Oct-4 and Sox- 2 were determined by Western analysis at the protein level or by qPCR at the mRNA level. 
Then, expression of the following members of miR-290 and miR-302 miRNA clusters was tested with quantitative reverse transcription PCR:

- miR-290 family: miR-290_5p, miR-291_3p, miR-292_3p, miR-293, miR-294 and miR-295.

- miR-302 family: miR-302a, miR-302b and miR-302d
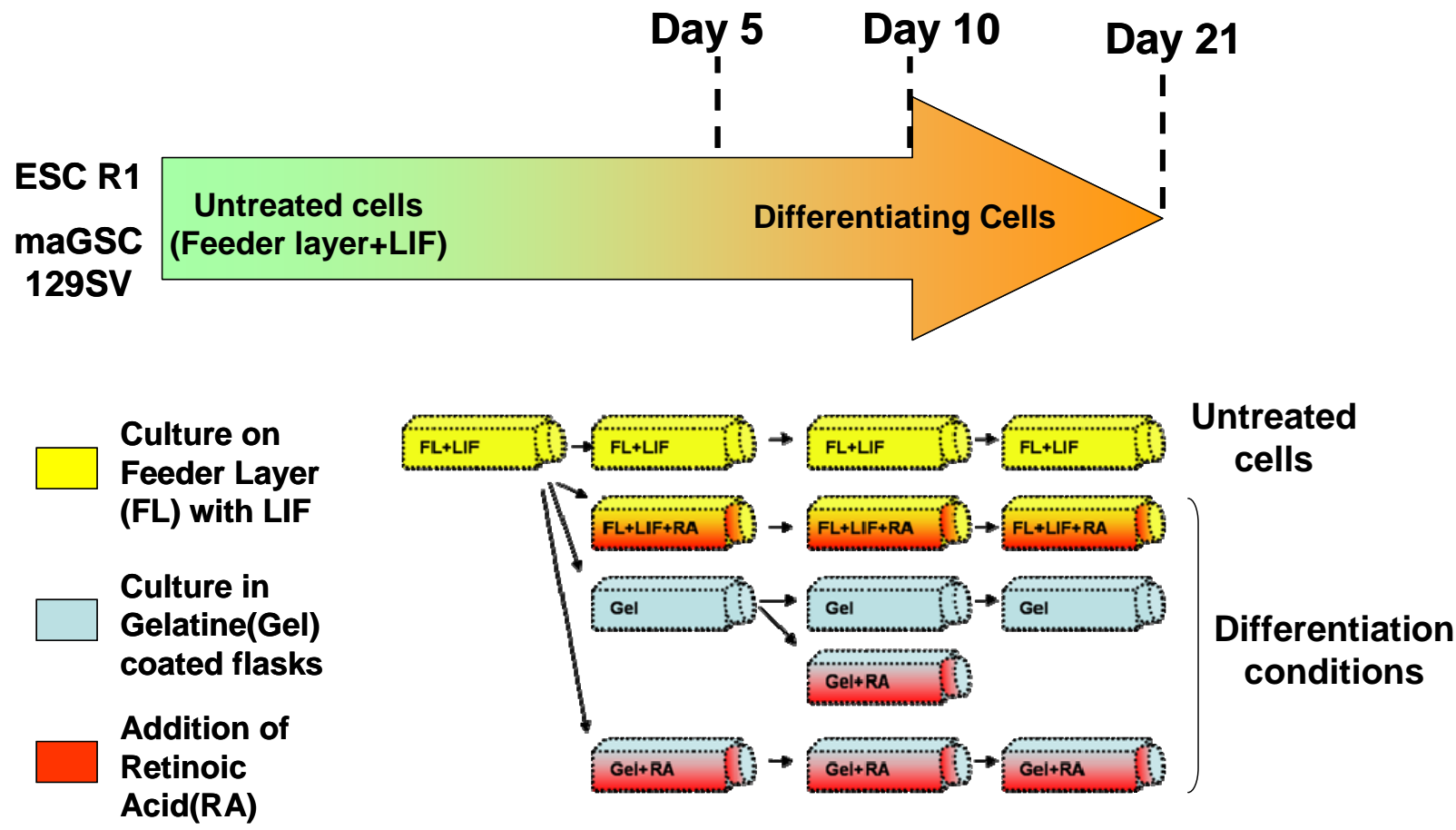

Figure 10. Cell culture strategy for in vitro differentiation of ESCs and maGSCs from 129/Sv mouse strain (ESC R1 and maGSC 129SV). Cells were cultured for 5,10 and 21 days under the different culture conditions mentioned in the text. Deprivation of LIF and culture in gelatin coated flasks is depicted with blue colour, while addition of RA with red. Cells cultured for the same period under standard ESC conditions (yellow) were used as control (untreated cells).

In case of F9 cells (ECCs), RA has been used in previous works (Alonso et al. 1991) to induce differentiation in these cells. Thus, in 
order to induce differentiation, cells were treated with $10^{-6} \mathrm{M}$ RA for 25 days (Fig. 11). Then cells were tested for the above mentioned miRNAs and pluripotency markers.

Day 25

\section{F9} cells
Untreated cells
Differentiating Cells

(addition of RA)

Figure 11. Cell culture strategy for in vitro differentiation of ECCs (F9 cells). Cells were cultured for 25 days in the presence of RA (orange). Cells cultured for the same period under standard conditions (green) were used as control (untreated cells).

\subsubsection{Determination of loss of pluripotency in treated cells}

Figure 12 shows that, after five days under FL+LIF+RA condition, expression of Oct-4 and Sox-2 is strongly downregulated in ESCs and maGSCs. After culture of the cells for five days under Gel+RA condition, Oct-4 expression is hardly detectable in maGSCs and absent in ESCs. Furthermore, no Sox-2 expression is detected.

However, culture under Gel condition for five days was found to result in downregulation of both pluripotency marker proteins only in ESCs, but not in maGSCs. In maGSCs, Oct-4 protein levels are similar to those of untreated cells. Only after cultivation of maGSCs under Gel condition for a longer period (21 days), Oct-4 expression is downregulated (Fig. 13). 

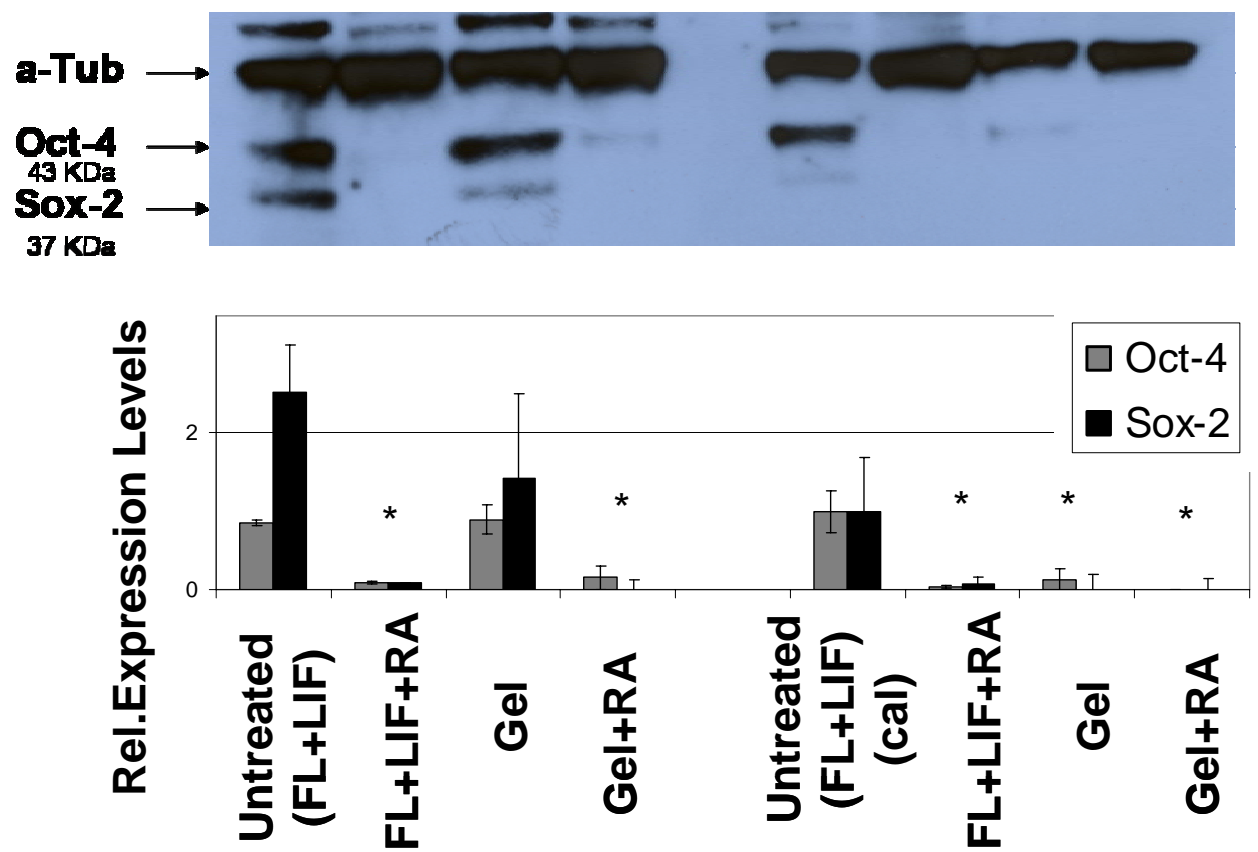

Figure 12. Expression of Oct-4 and Sox-2 proteins by Western blotting in ESCs and maGSCs cultured for five days under the above mentioned conditions. Quantification of the blots was done by densitometry. Expression levels were compared between untreated and differentiating cells, asterisks indicate statistical significance. a-Tubulin served as loading control and for normalization of the values. Untreated ESCs were used as calibrator.

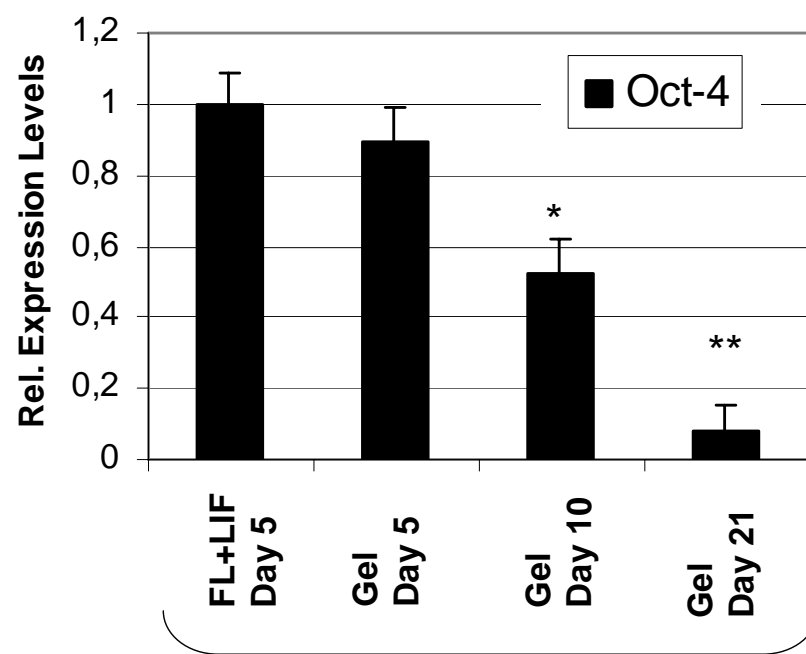

maGSC

129SV
Figure 13. Expression of Oct-4 protein in untreated maGSCs (FL+LIF/calibrator) and in maGSCs under Gel condition over time (5, 10 and 21 days). Quantification of Western blotting results was done as described previously. One asterisk indicates statistical significance between day 5 and day 10, two asterisks between day 10 and day 21 . 


\section{FL+LIF+RA}

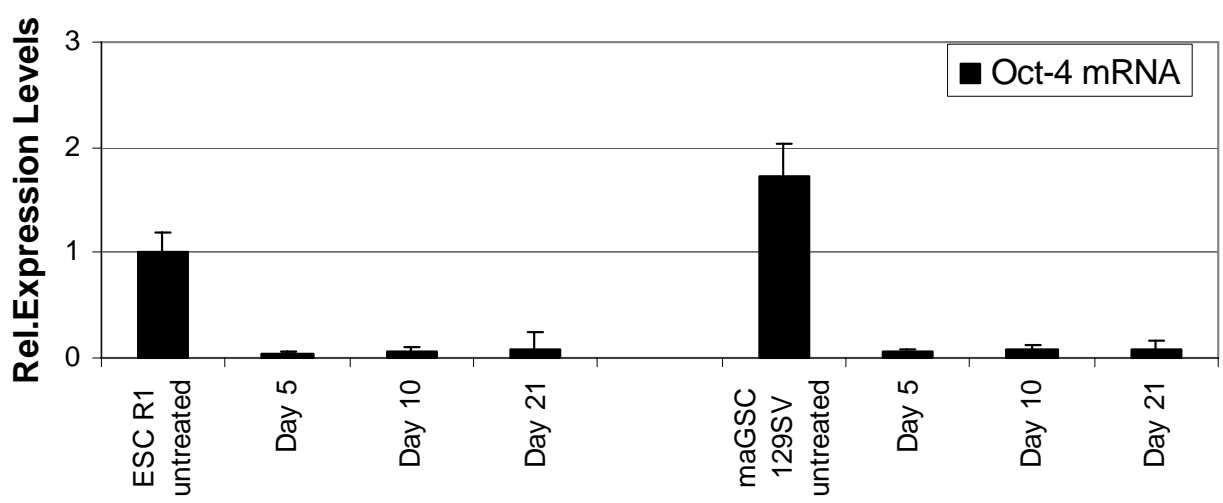

\section{Gel}

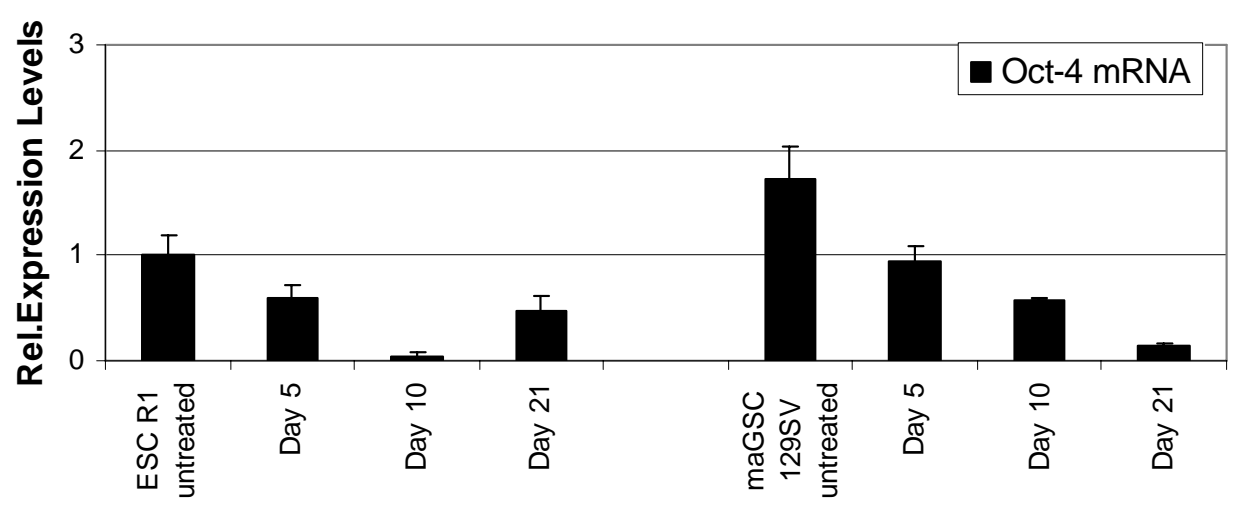

Gel+RA

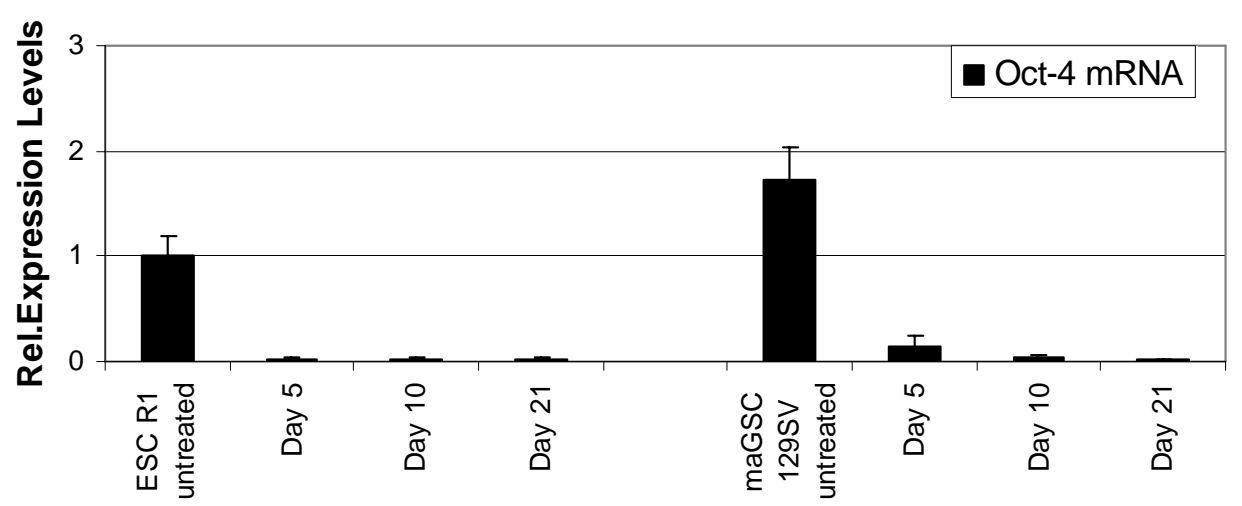

Figure 14. Expression of Oct-4 mRNA by Real-time PCR in ESCs and maGSCs cultured for 5, 10 and 21 days under all three differentiation conditions compared with untreated cells. Expression levels were normalized to endogenous control gene (Sdha) and calibrated to the levels of untreated ESC R1. 
Evaluation of Oct-4 expression at the mRNA level confirmed the findings of Western blotting and revealed an upregulation of Oct-4 in ESCs under Gel condition at day 21 (Fig. 14).

Also in case of F9 cells, downregulation of Oct-4 mRNA levels after treatment with RA for 25 days confirmed successful induction of differentiation for these cells (Fig.15).

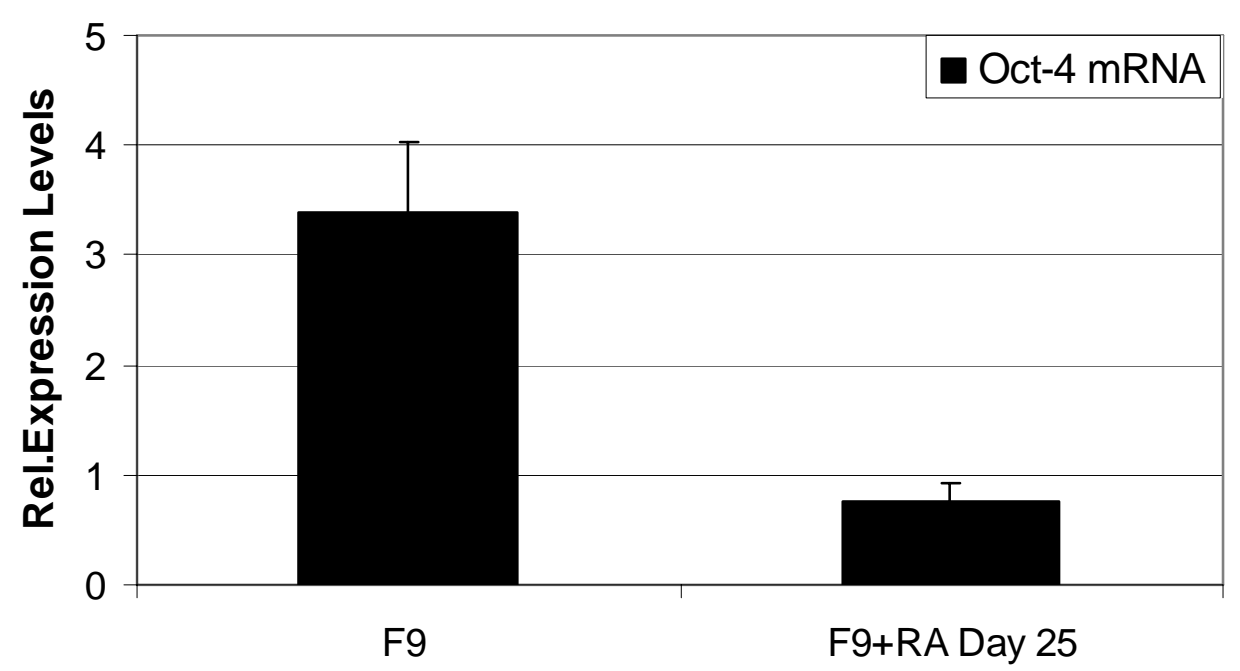

Figure 15. Expression of Oct-4 mRNA by Real-time PCR in F9 cells treated with RA compared with untreated cells. Expression levels were normalized and calibrated as in Figure 14.

\subsubsection{Expression profile of miR-290 cluster during in vitro differentiation of ESCs and maGSCs}

Figure 16 shows the timeline expression profile of miRNAs of the 290 miRNA cluster in ESCs and maGSCs under all differentiation conditions compared with untreated cells. Expression profiles where the different conditions for each miRNA are superimposed to facilitate their comparison are also presented in Appendix 3A. 


\section{FL+LIF+RA}

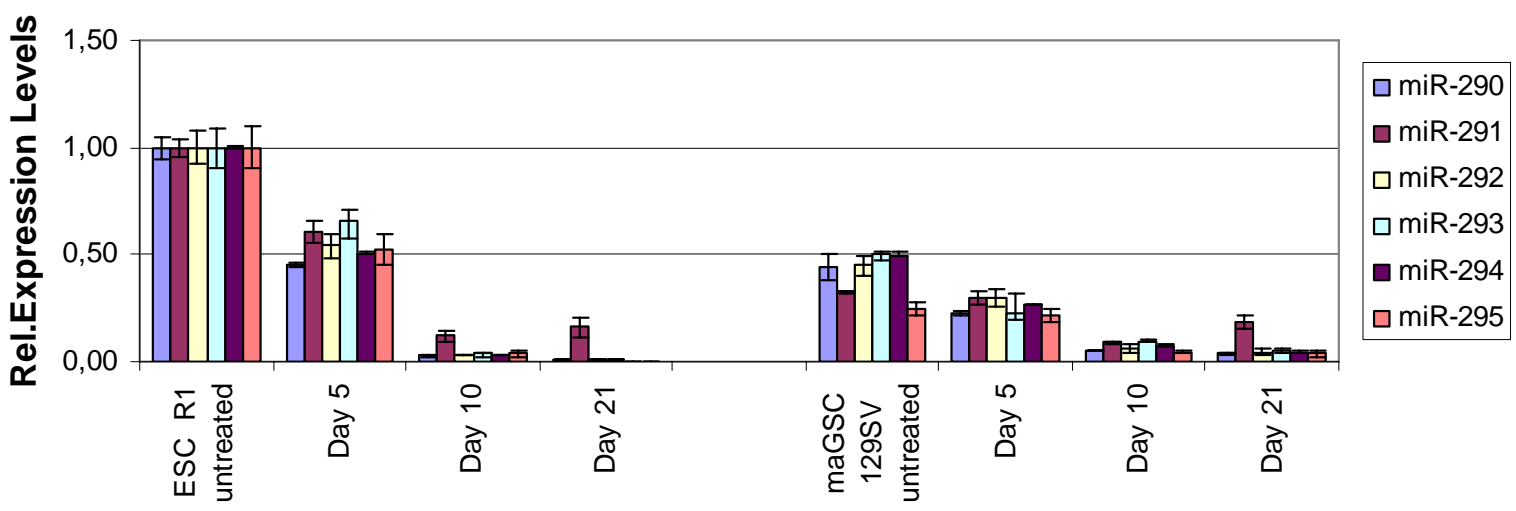

Gel

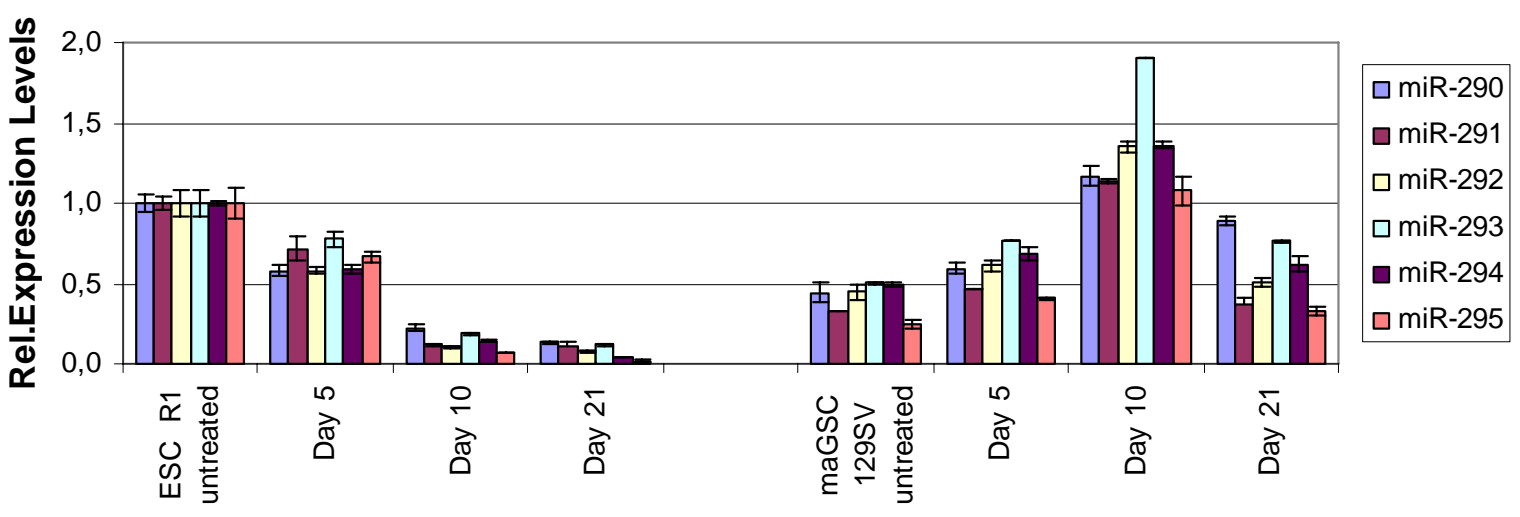

Gel+RA

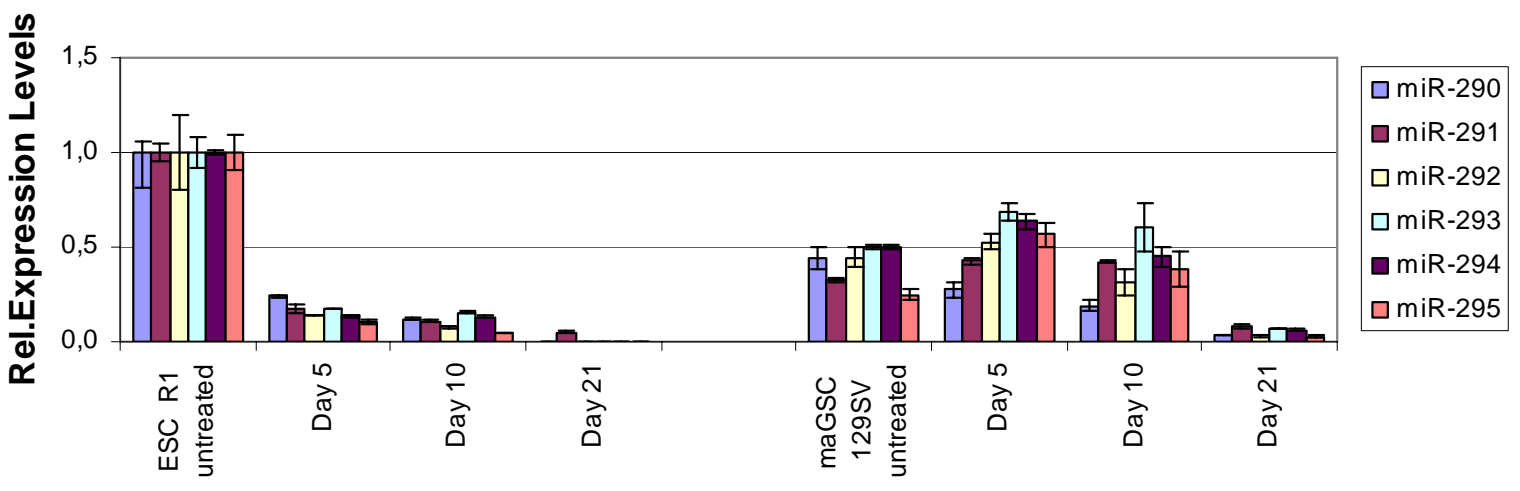

Figure 16. Expression profiles of members of the miRNA 290 family in ESCs and maGSCs from mouse strain 129/Sv (ESC R1 and maGSC 129SV) under different differentiation conditions after 5,10 and 21 days in culture compared with untreated cells. All levels were normalized to endogenous control (RNU6B) and calibrated to the value of untreated ESC R1. For statistical significance of the observed differences see Appendix 4A. 
In both ESCs and maGSCs, all members of the 290-family are constantly expressed or even increased in untreated cells, although in case of maGSCs at lower levels (50\% of ESC expression in some cases) comparing with ESCs. However, miRNA levels vary even between cells cultured under standard ESC conditions but for different periods of time (for example in case of miR-293 an increase in expression levels of more than $50 \%$ in untreated ESCs at day 21 compared with untreated ESCs at day 5 is observed).

In ESCs, these miRNAs are downregulated at day 5 of culture under all differentiation conditions with the strongest effect observed in Gel+RA condition. At day 10 and 21, miRNA levels can hardly be detected under all differentiation conditions.

In maGSCs at day 5, levels of miRNAs do not decrease in Gel and Gel+RA conditions (with the exception of miR-290 in Gel+RA). Their expression is the same or even higher than in untreated cells. At day 10, miRNA levels have further increased under Gel condition (Fig.6b). In Gel+RA condition at day 10, miRNA levels do not increase further but they are still high, whereas a strong downregulation at day 10 is observed only under FL+LIF+RA condition. At day 21, miRNA levels of cells in Gel condition are lower than those of untreated cells (with the exception of miR-290), but remain still high in comparison to the other two conditions (Gel+RA and FL+LIF+RA), where miRNAs are hardly detectable.

\subsubsection{Expression profile of miR-302 cluster during in vitro differentiation of ESCs and maGSCs}

The expression profiles of members of the 302-cluster were found to differ significantly from those of miR-290 family members (Fig. 17, Appendix 3B). 


\section{FL+LIF+RA}

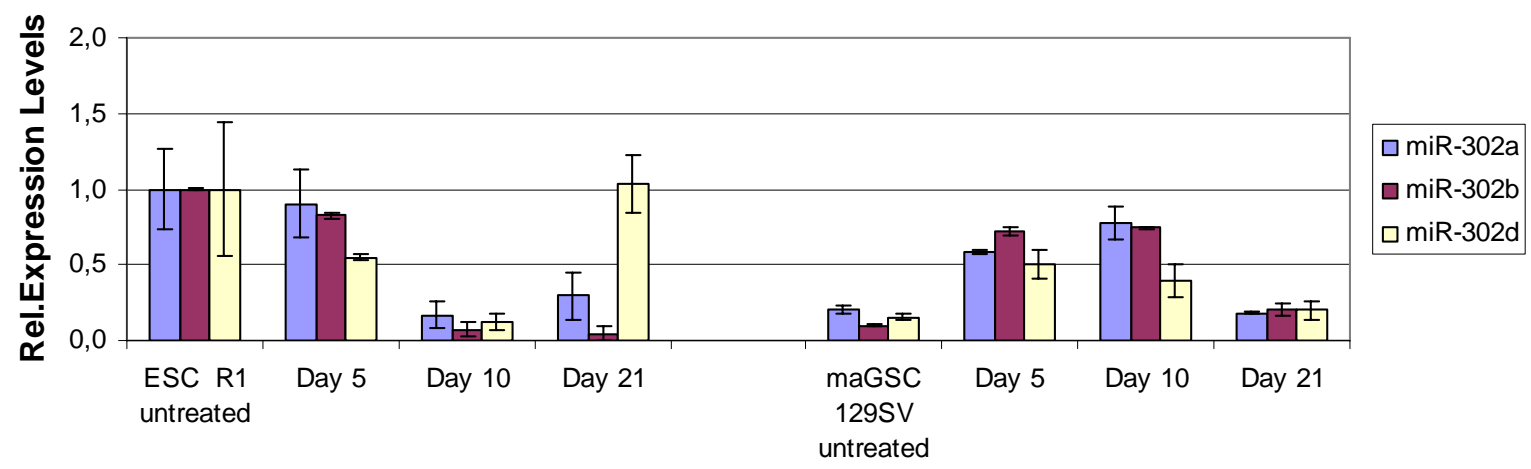

Gel

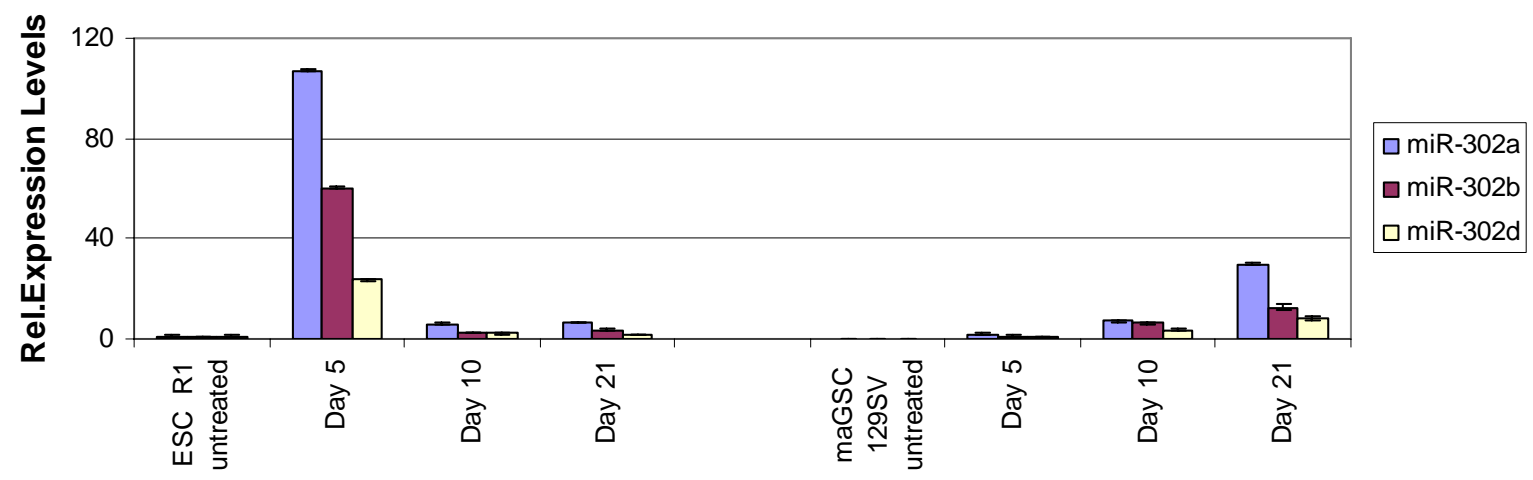

Gel+RA

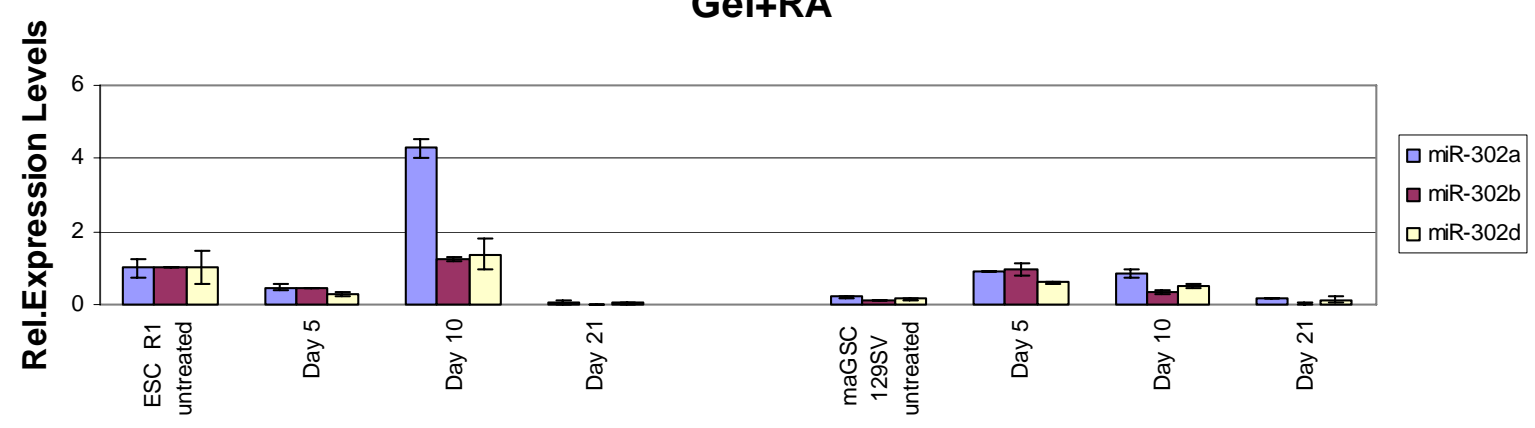

Figure 17. Expression profiles of members of the miRNA 302 family in ESCs and maGSCs from mouse strain $129 / \mathrm{Sv}$ under different differentiation conditions after 5, 10 and 21 days in culture compared with untreated cells. Levels were normalized and calibrated as in Figure 16. For statistical significance of the observed differences see Appendix 4B. 
In ESCs, the Gel condition has an extreme effect on the expression of miRNAs 302 at day 5. They become strongly upregulated (20-100 fold increase). Their levels decrease rapidly after day 5, but even at days 10 and 21 of culture, the miRNA expression is still higher than in untreated cells. In Gel+RA, miRNA levels increase temporally to levels higher than in untreated cells around day 10. Then they decrease leading to expression levels lower than in untreated cells at day 21. miRNAs 302 become also upregulated in maGSCs under Gel condition. During the culture period of 21 days, expression levels increase 10-30 fold. However, in case of maGSCs, levels increase gradually at least until day 21, and not only at day 5 like in ESCs.

In the other two conditions (Gel+RA and FL+LIF+RA) miRNA levels at day 5 are higher than those in untreated cells and become similar to them thereafter.

\subsubsection{Expression profile of $\mathrm{miR}-290$ and $\mathrm{miR}-302$ clusters during differentiation of ECCs}

When ECCs are treated with $10^{-6}$ M RA for 25 days, miR-290 and miR-291 levels decrease slightly, miR-292, miR-293 and miR-294 levels remain relatively stable and only miR-295 increases. In contrast, levels of all miR-302 family members increase significantly (3-5 fold increase) (Fig. 18). 


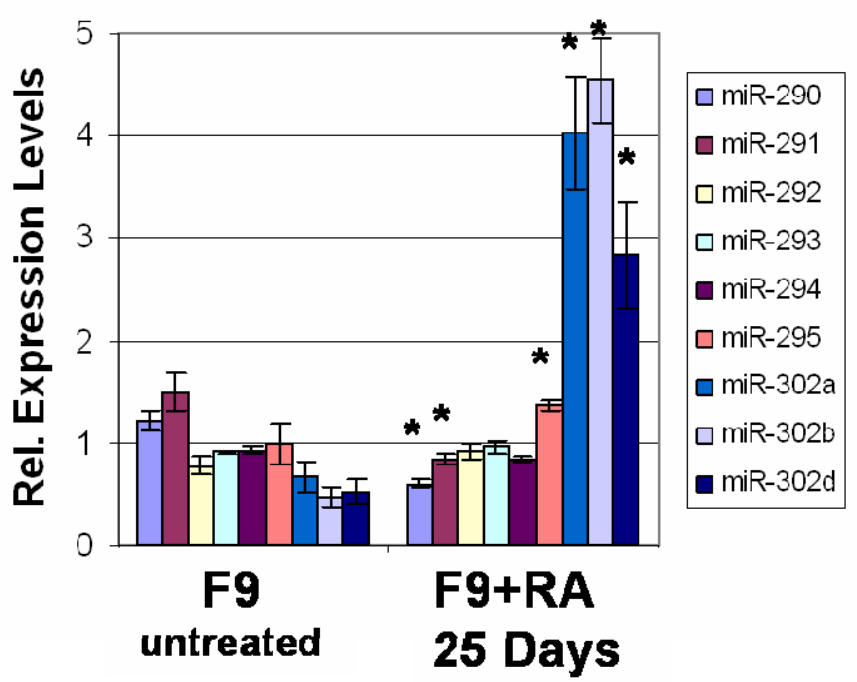

Figure 18. Expression profiles of members of miRNA families 290 and 302 in untreated F9 cells and in those treated with RA for 25 days. All levels were calibrated to the value of untreated ESC R1 (FL+LIF) at day 5. Asterisk depicts statistical significance. 


\subsection{Determination of the differentiation state of the cells tested}

In ESCs, there is a body of citable evidence that provides a precedent for using these methods to differentiate ESCs in vitro. To test whether maGSCs achieve like ESCs multilineage differentiation in the differentiation system employed in this study, differentiation into three germ layers was tested. This was performed by analyzing the time-course and extent of differentiation marker expression in the maGSCs cultured under the various conditions. Expression of the following differentiation markers was tested with qPCR:

- Nestin (for ectoderm)

( Lendahl, 1997; Lin et al., 1995; Wiese et al., 2004)

- Brachyury (for mesoderm)

(Showell et al., 2003)

- Hnf-4 (for endoderm)

(Duncan et al., 1997; Taraviras et al., 1994)

- Nkx-2.5 (for early embryo heart formation)

(Liberatore et al., 2002)

Expression levels in the respective ESCs were also evaluated for control and comparison with maGSCs. For statistical significance of the observed differences (with the exception of Brachyury, where all differences were statistical significant when it was expressed) see Appendix 5. 


\subsubsection{Ectoderm formation: Nestin}

maGSCs differentiate towards ectoderm later than ESCs, with the exception of FL+LIF+RA condition, where only maGSCs express Nestin in higher levels compared with untreated cells (Fig. 19).

\subsubsection{Mesoderm formation: Brachyury}

No Brachyury expression was observed in both cell types when RA was added. Under Gel condition ESCs express Brachyury at high levels at day 5 and then again at day 21. In contrast, maGSCs express Brachyury later than ESCs, at day 21 and in lower levels (Fig. 20).

\subsubsection{Endoderm formation: Hnf-4}

Undifferentiated maGSCs express Hnf-4 at higher levels than undifferentiated ESCs. In both cell types upregulation applies to conditions where RA is added. In contrast to maGSCs, in ESCs upregulation occurs only transiently at day 5 and at lower levels (Fig. 21).

\subsubsection{Early embryo heart formation: Nkx-2.5}

In ESCs upregulation occurs mainly under Gel+RA condition and under Gel condition at day 21. In maGSCs upregulation occurs only under Gel condition after day 5 at similar levels with ESCs (Fig. 22). 


\section{LIF+RA}

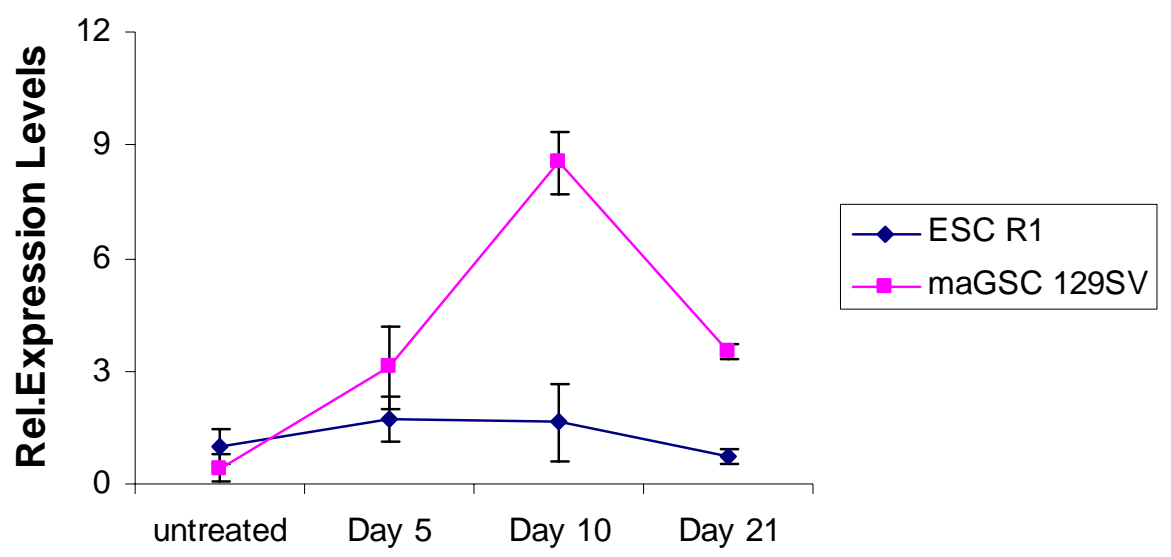

\section{Gel}

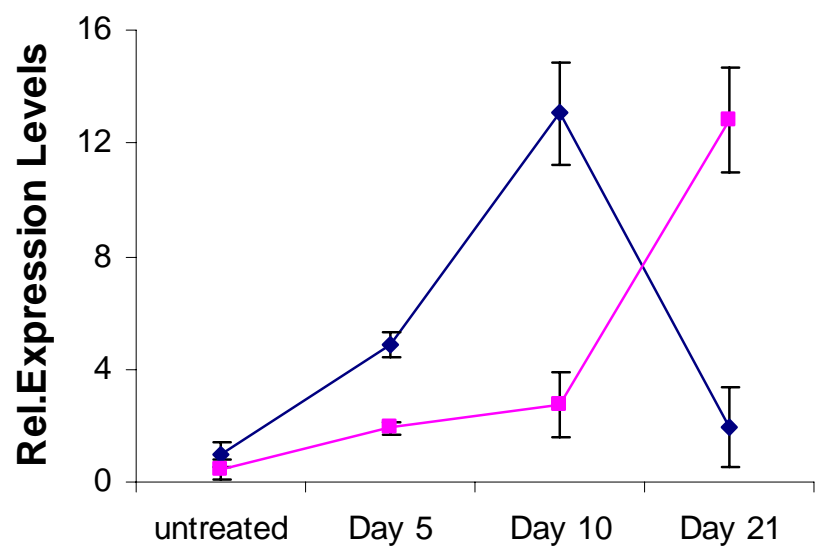

\section{Gel+RA}

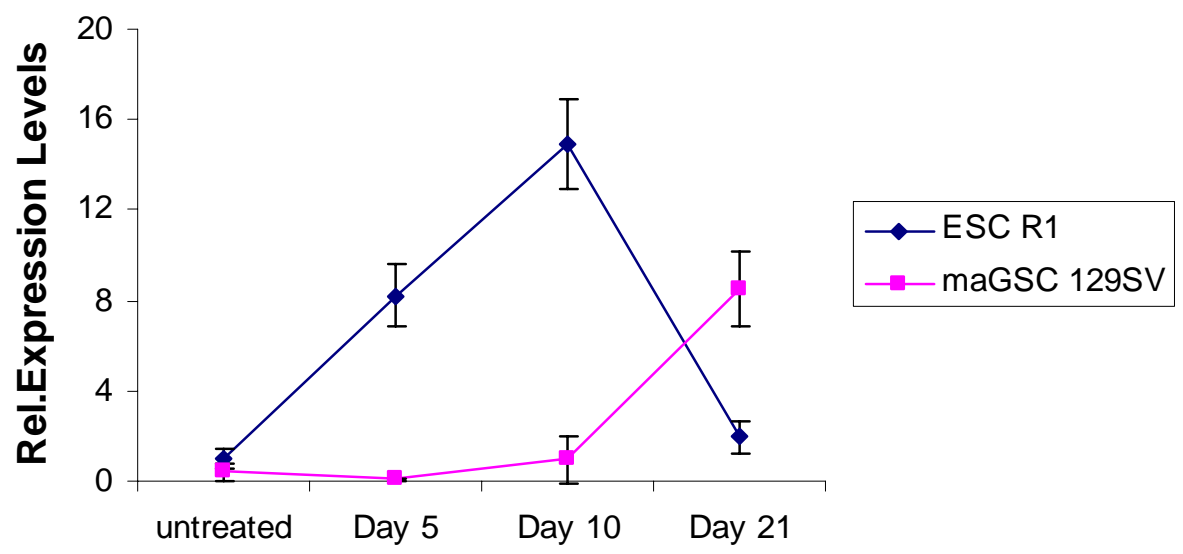

Figure 19. Expression of Nestin mRNA by Real-time PCR in ESCs and maGSCs cultured for 5, 10 and 21 days under all three differentiation conditions compared with untreated cells. Expression levels were normalized to endogenous control gene (Sdha) and calibrated to the levels of untreated ESC R1. 


\section{LIF+RA}

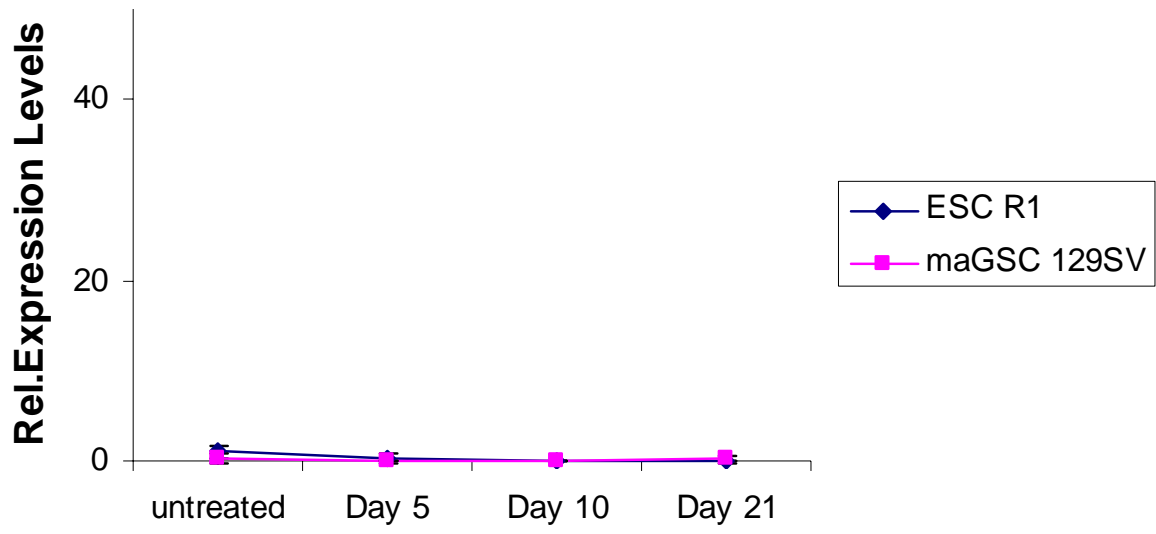

Gel

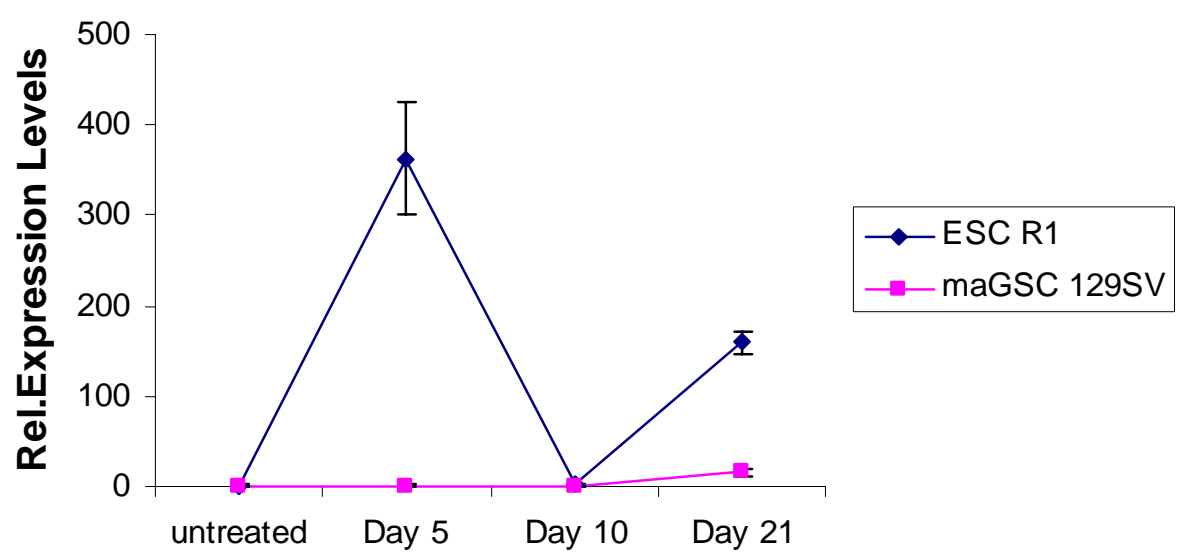

Gel+RA

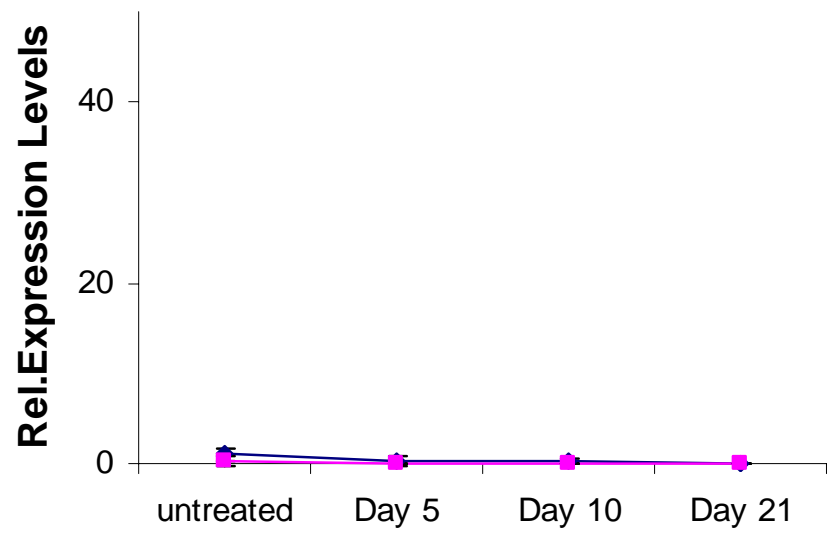

Figure 20. Expression of Brachyury mRNA by Real-time PCR in ESCs and maGSCs cultured for 5, 10 and 21 days under all three differentiation conditions compared with untreated cells. Expression levels were normalized and calibrated as in Figure 19. 


\section{LIF+RA}

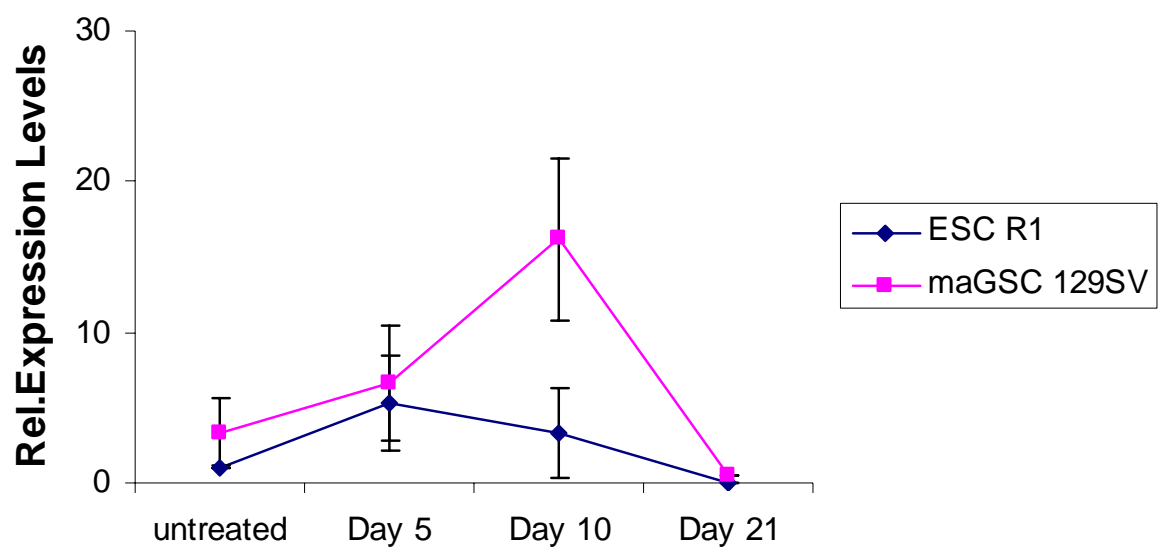

Gel

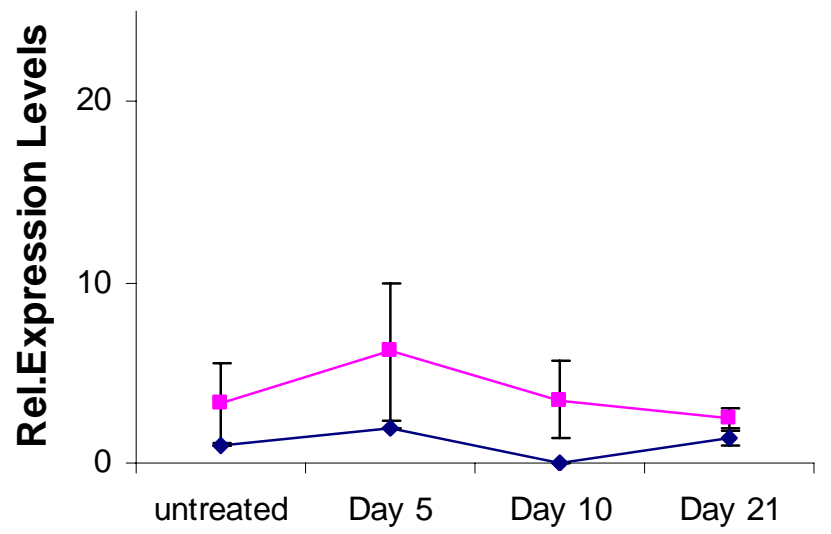

Gel+RA

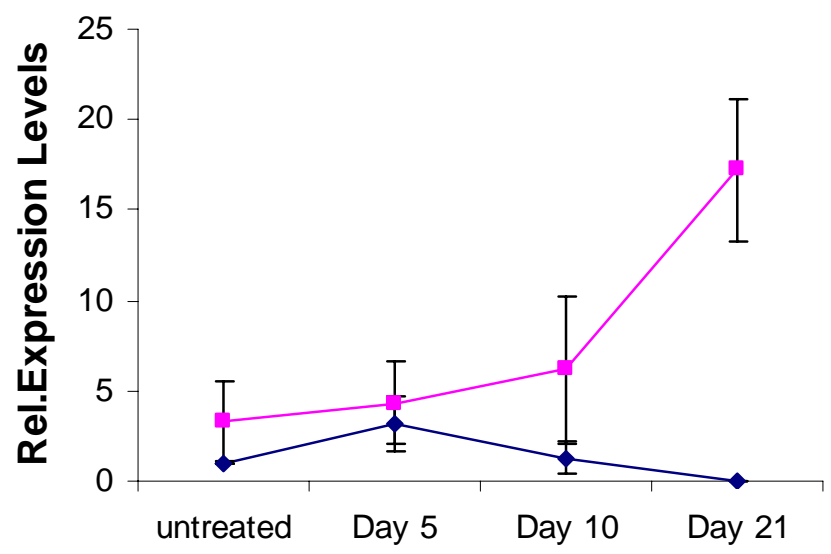

Figure 21. Expression of Hnf-4 mRNA by Real-time PCR in ESCs and maGSCs cultured for 5, 10 and 21 days under all three differentiation conditions compared with untreated cells. Expression levels were normalized and calibrated as in Figure 19. 


\section{LIF+RA}

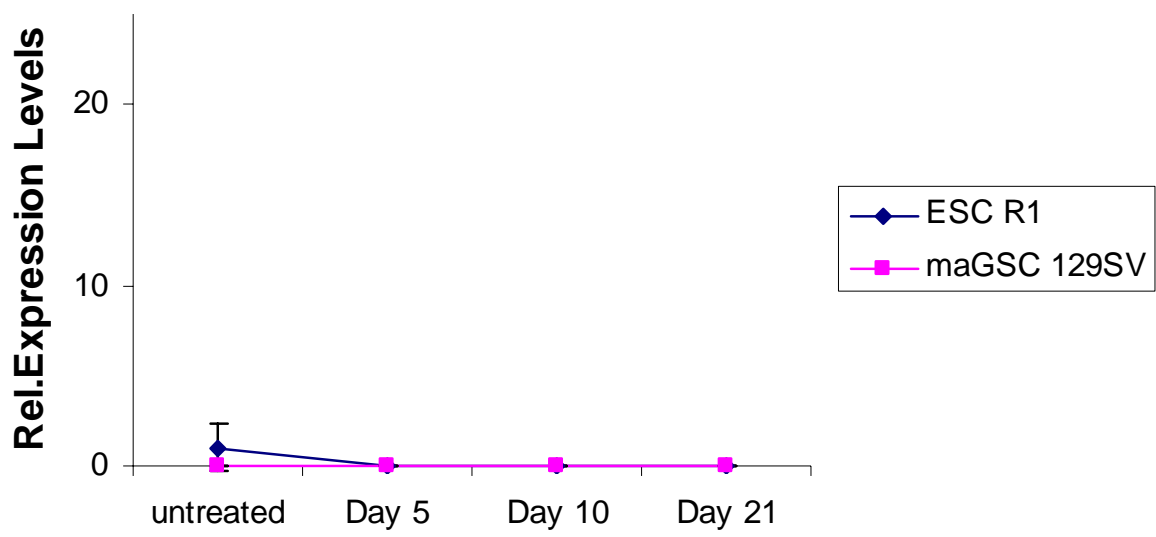

\section{Gel}

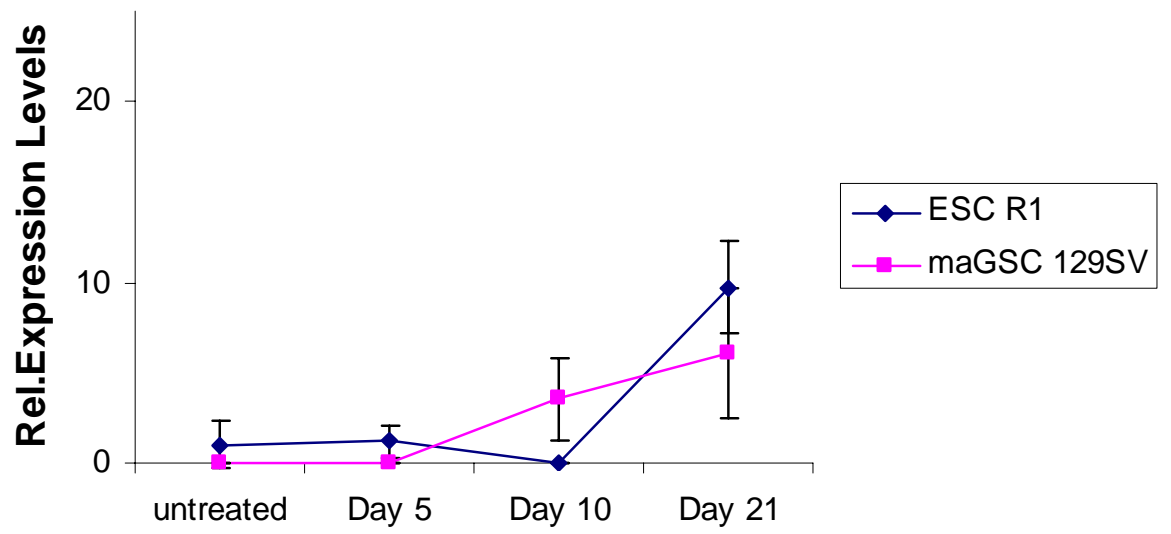

Gel+RA

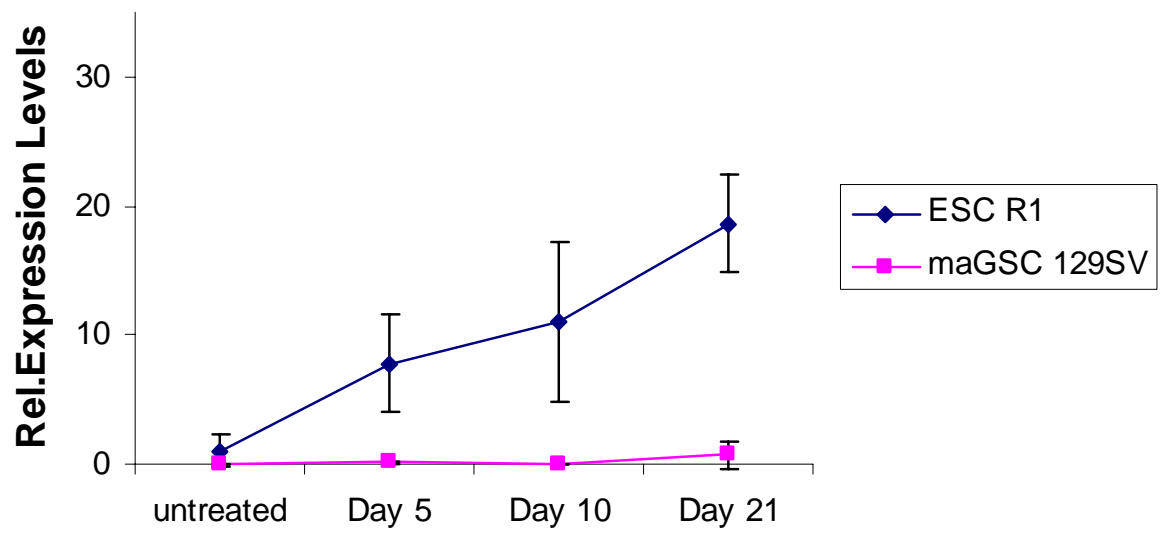

Figure 22. Expression of Nkx-2.5 mRNA by Real-time PCR in ESCs and maGSCs cultured for 5, 10 and 21 days under all three differentiation conditions compared with untreated cells. Expression levels were normalized and calibrated as in Figure 19. 


\subsubsection{Differentiation state of treated F9 cells}

Differentiation of F9 cells treated with RA was characterized by an increase in Nestin and Hnf-4 expression (Fig. 23), while no expression was detected for Brachyury (data not shown).

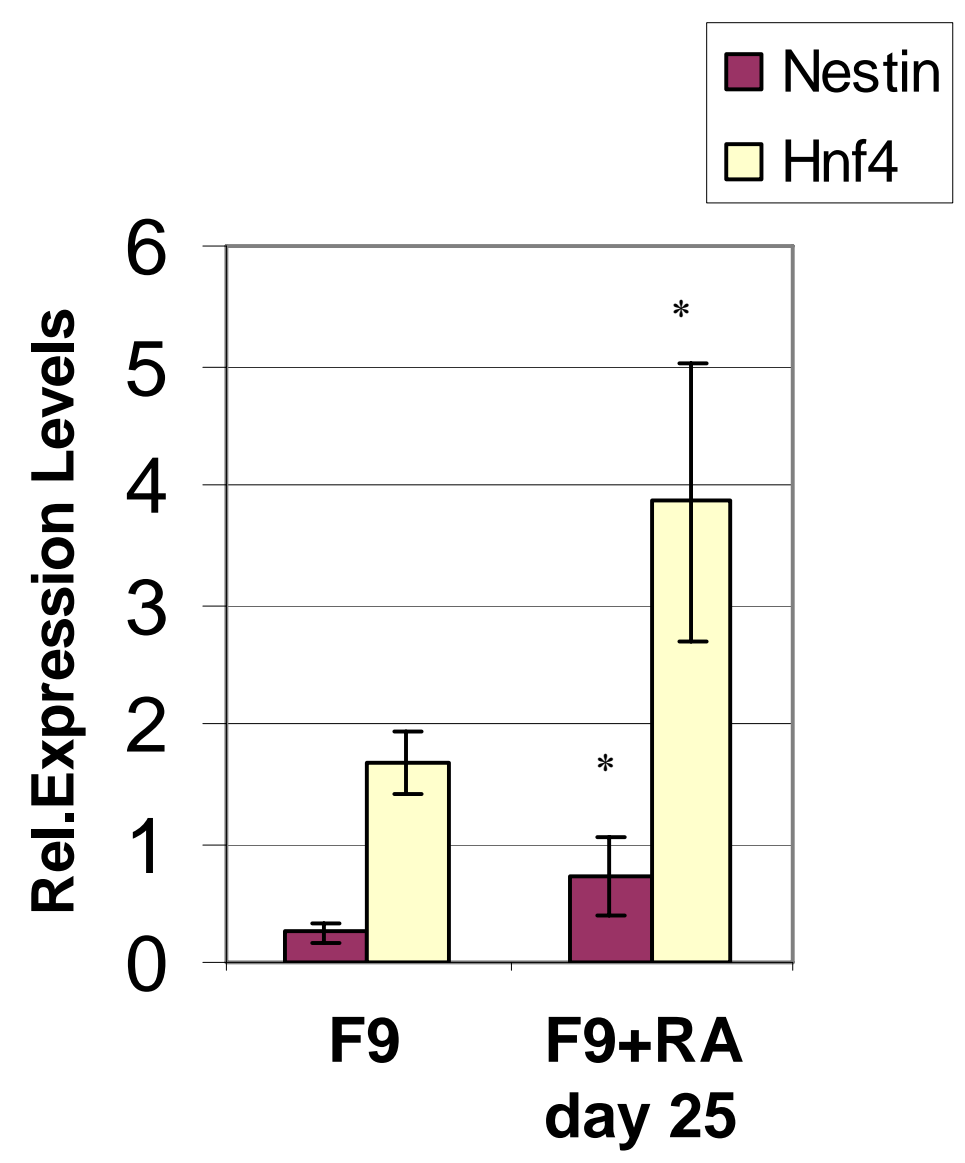

Figure 23. Expression of Nestin and Hnf-4 in untreated and treated with RA F9 cells. Asterisk depicts statistical significance. Expression levels were normalized and calibrated as in Figure 19. 


\subsection{Correlation of the differentiation state of the cells with miRNA levels of miR-290 and miR-302 clusters.}

In order to identify a possible connection between expression levels of miRNAs tested and differentiation state, differences in miRNA levels between the different cell types and different conditions tested were correlated with differences in the expression of pluripotency and differentiation markers. Based on these comparisons the following correlations were found:

\subsubsection{Correlation of miR-302 with Brachyury expression}

Figure 24 shows that in both ESCs and maGSCs, changes in the miRNA levels of miR-302 family follow the same pattern as changes in the Brachyury expression. 
miR-302 in ESC R1
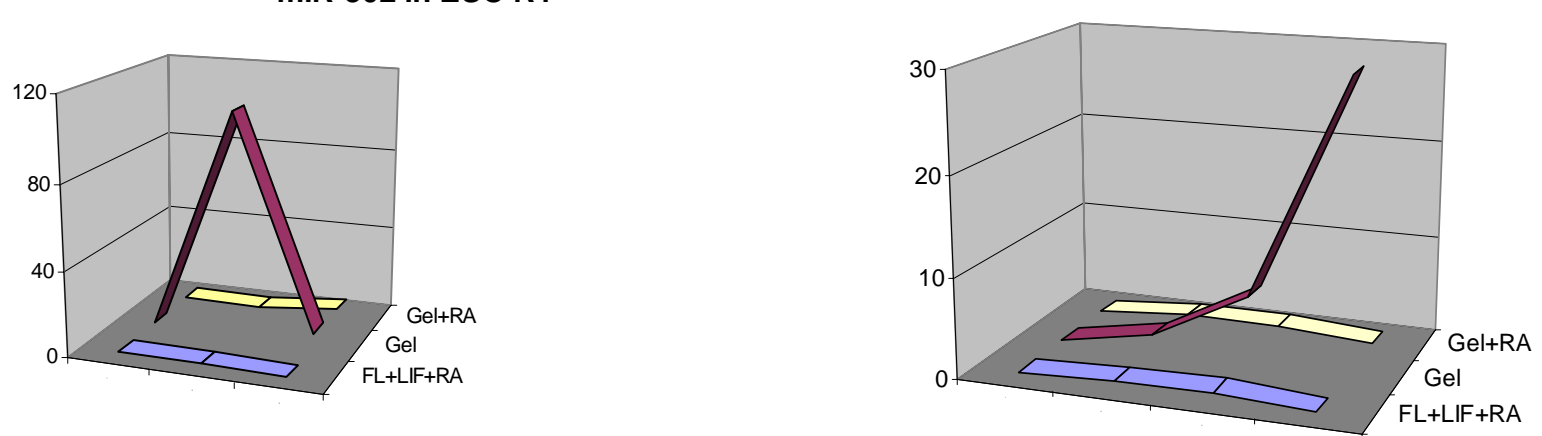

Brachyury in ESC R1
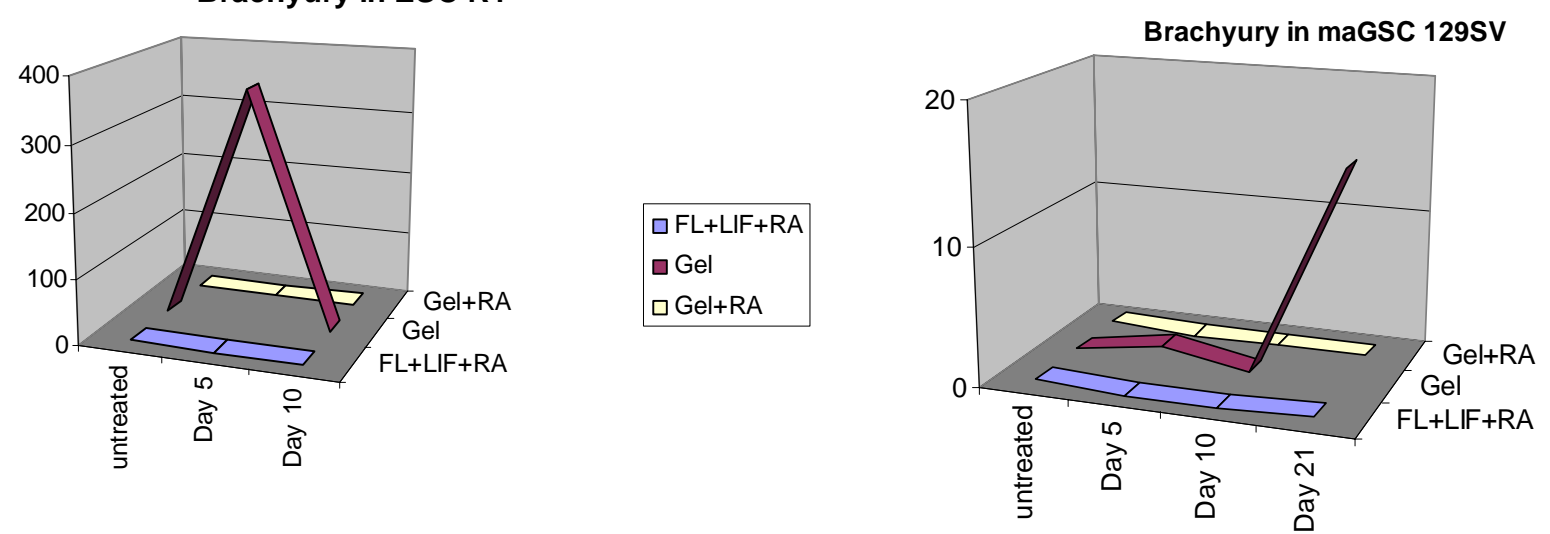

Figure 24. Relative expression levels (qPCR) of miR-302a (up) and Brachyury (down) in ESC R1 (left) and maGSC 129SV (right). A significant increase in Brachyury and miR-302 expression is observed (in both cell types) only in Gel condition

\subsubsection{Correlation of miR-290 with Oct-4 expression (based on differences between different conditions)}

In both ESCs and maGSCs, higher levels in miR-290 cluster levels under Gel condition compared with the other two conditions are connected with also higher levels of Oct-4 under this condition (Fig. 25). 
miR-290 in ESC R1

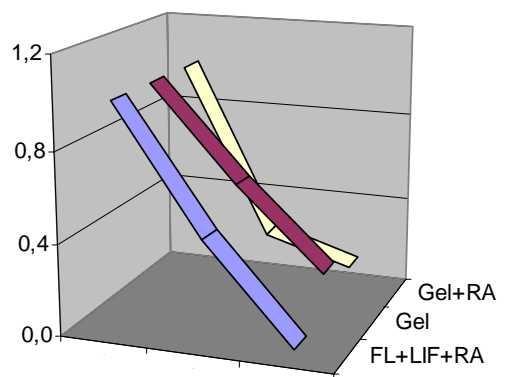

Oct-4 in ESC R1

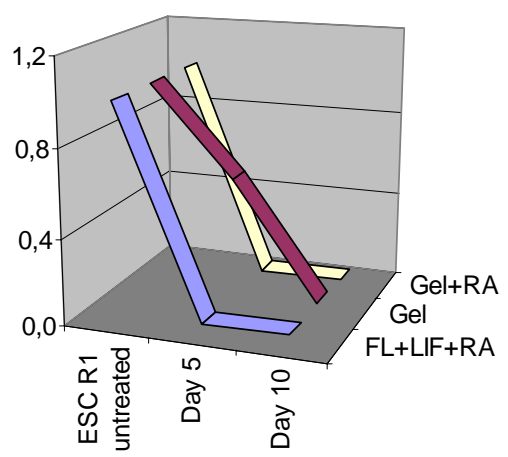

miR-290 in maGSC 129SV

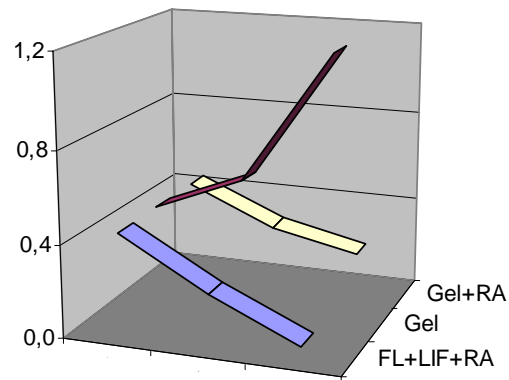

Oct-4 in maGSC 129SV

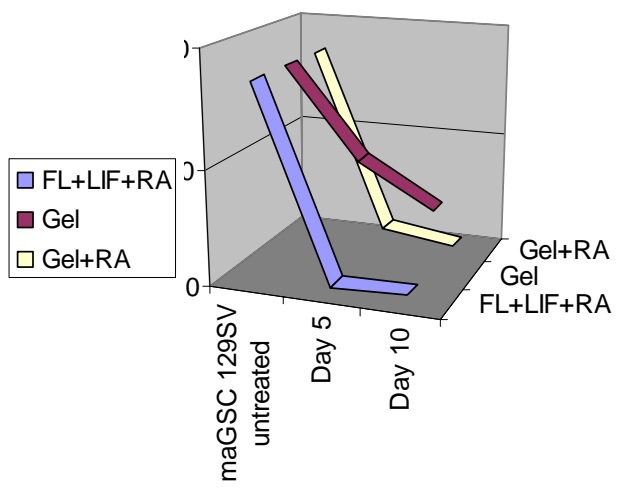

$\square \mathrm{FL}+\mathrm{LIF}+\mathrm{RA}$

$\square \mathrm{Gel}$

$\square$ Gel+RA

Figure 25. Relative expression levels (qPCR) of miR-290 (up) and Oct-4 (down) in ESC R1 (left) and maGSC 129SV (right). High levels in Oct-4 and miR-290 expression are observed (in both cell types) only under Gel condition.

\subsubsection{Correlation of miR-290 with Oct-4 expression and differentiation (based on differences between different cell types)}

Under Gel and/or Gel+RA condition, Nestin and Brachyury become upregulated earlier in ESCs as compared with maGSCs, while the opposite characterizes Oct-4 and miR-290 cluster expression, which in maGSCs decrease later than in ESCs (Figs. 12, 16, 19 and 20). 


\subsubsection{Time dependent influence of RA during ESC and maGSC differentiation}

Effects of RA on differentiation have been shown to be time dependent. Thus, miRNA and differentiation markers expression levels at day 10 were compared between Gel+RA (or Gel+RA from day 0) condition (where RA was added from the beginning of differentiation) and Gel+RA from day 5 (where RA was added from day 5 of differentiation onwards).

In maGSCs, if RA is added from day 5 onwards (Gel+RA from day 5), 290 miRNA family levels at day 10 are lower as compared with Gel+RA condition (Fig. 26). In addition, the increase from day 5 to day 10 observed in maGSCs under Gel for miR-302 family is minimized when RA is added from day 5 onwards (Gel+RA from day 5 ), but miRNA levels in this condition are still higher as compared with Gel+RA at the same day (Fig. 27).

miR-291

maGSC 129SV

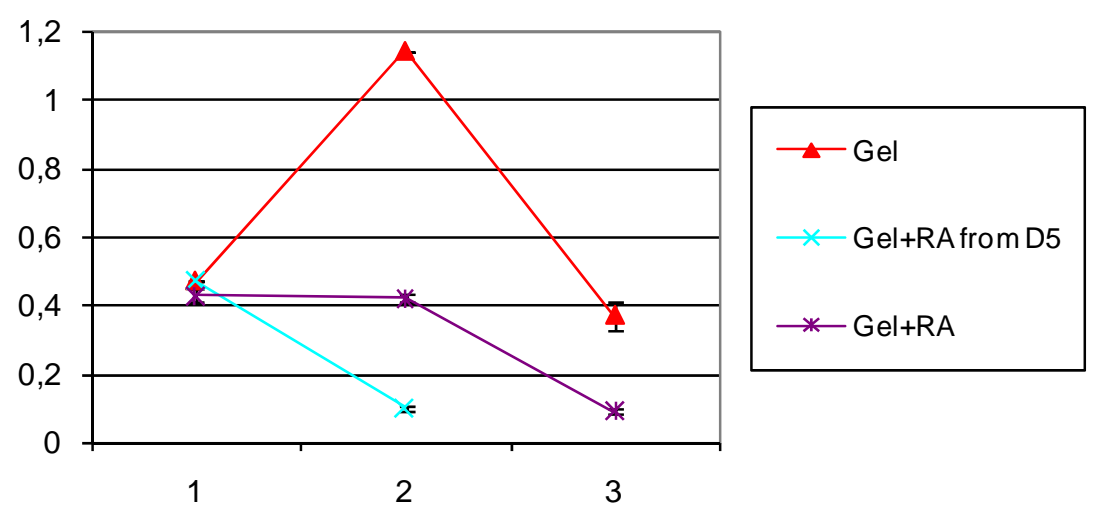

Figure 26. miR-291 expression levels at day 5(1), 10(2) and 21(3) under the three differentiation conditions mentioned in the text. Similar patterns were observed for the other members of miR-290 cluster and are presented in Appendix 3A). 
miR-302b

maGSC 129SV

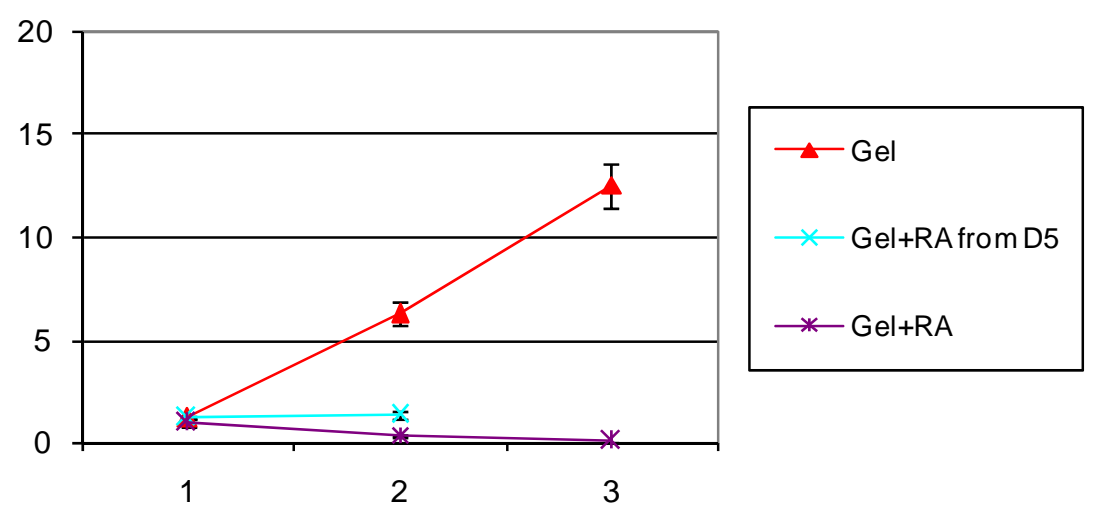

Figure 27. miR-302b expression levels at day 5(1), 10(2) and 21(3) under the three differentiation conditions mentioned in the text. Similar patterns were observed for the other members of miR-302 cluster and are presented in Appendix 3B).

Finally, in ESCs, miRNA levels of 302 cluster under Gel+RA from day 5 condition are significantly lower than Gel+RA from day 0 condition (Fig. 28). 
a

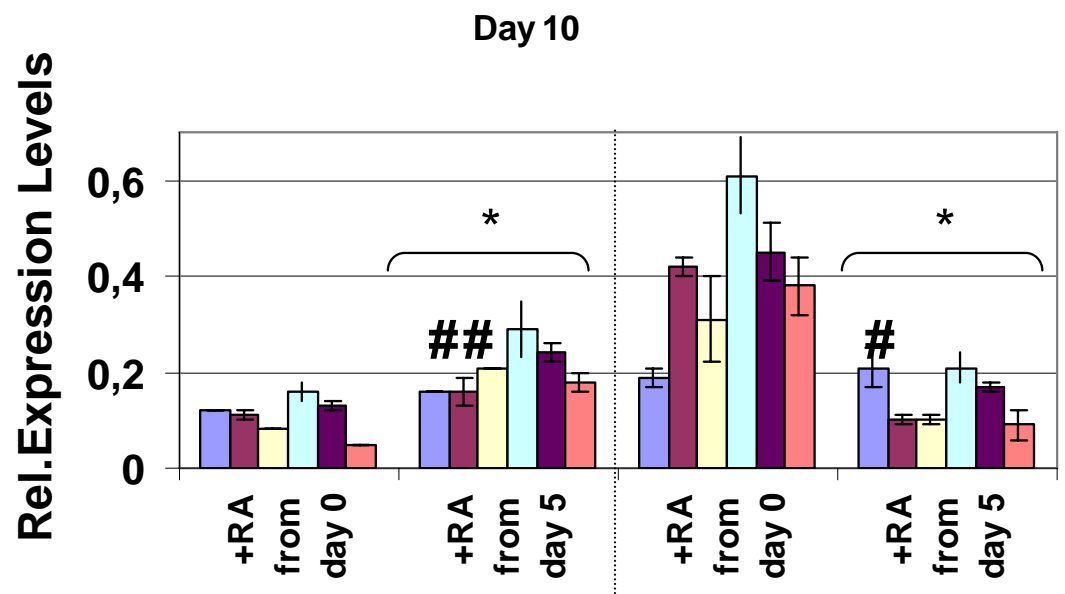

$\overline{\mathrm{miR}-290}$

$\square$ mir-291

$\square$ miR-292

$\square$ miR-293

口 miR-294

$\square$ miR-295

b

Day 10

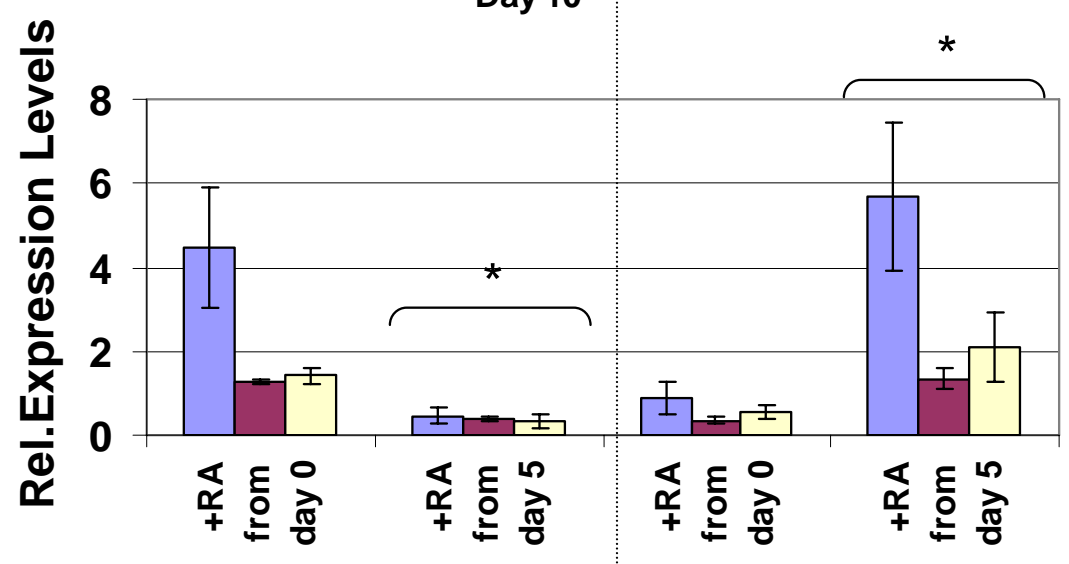

$\square$ miR-302a

$\square$ miR-302b

$\square$ miR-302d

Day 10

C

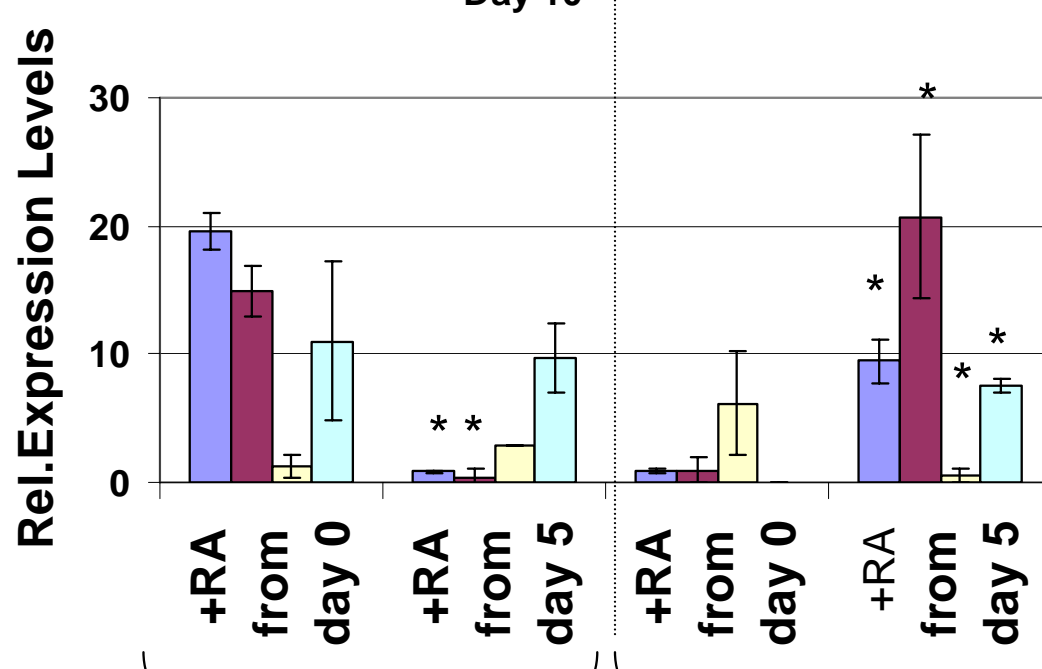

$\square$ Vimentin

$\square$ Nestin

$\square \mathrm{Hnf4}$

$\square \mathrm{Nk} \times 2.5$

ESC R1 on Gel
maGSC

129SV

on Gel

Figure 28 
Figure 28. Comparison of expression levels of members of miRNAs and differentiation markers at day 10 in ESCs and maGSCs from mouse strain 129/Sv (ESC R1 and maGSC 129SV) between Gel+RA (cells cultured in Gel coated flasks with addition of RA from the beginning) and Gel+RA from day 5 (cells cultured in Gel coated flasks with addition of RA from day 5) conditions. The line that cuts the diagram in two parts, separates ESCs (left) from maGSCs (right). All miRNA and mRNA levels were normalised to endogenous control (RNU6B and Sdha respectively) and calibrated to the value of untreated ESC R1 $(\mathrm{FL}+\mathrm{LIF})$ at day 5. Asterisk indicates statistical significance comparing with Gel +RA from day 0 condition. \# indicates no statistical significance (a) Expression levels of miR-290 family. (b) Expression levels of miR-302 family. (c) Expression levels of Vimentin, Nestin, Hnf-4 and Nkx-2.5

Concerning differentiation markers, Vimentin, a marker expressed early during gastrulation, was also tested (Boisseau and Simonneau 1989; Colucci-Guyon et al. 1999; Franke et al. 1982). Compared with Gel+RA condition, levels of Nestin and Vimentin in ESCs were lower when RA was added from day 5, while in maGSCs Nestin, Vimentin and Nkx-2.5 were increased under this condition and only Hnf-4 levels were lower (Fig. 28).

In general, when Gel+RA and Gel+RA from day 5 conditions are compared, high levels of miR-290 family are connected with low levels of miR-302 family and most of differentiation markers (with the exception of Hnf-4) and vice versa. 


\subsection{Determination of de novo DNA methyltransferases expression profile in ESCs and maGSCs}

De novo DNA methyltransferases 3A and 3B (Dnmt3A and 3B) have been shown recently to be regulated by miRNAs of the 290 cluster by controlling an inhibitor of their transcription, Rbl protein. In order to test whether differences in 290 miRNA expression profile between ESCs and maGSCs during differentiation affect expression of Dnmts, their levels were determined during in vitro differentiation of both cell types.

As shown in Figure 29, expression profile of Dnmt3A differed between ESCs and maGSCs in all three differentiation conditions. Levels of Dnmt3A in maGSCs are downregulated during differentiation, while in ESCs DNMT3A is upregulated under Gel and Gel+RA condition. In contrast Dnmt3B showed a similar expression pattern between the two cell types (Fig. 30). 


\section{LIF+RA}

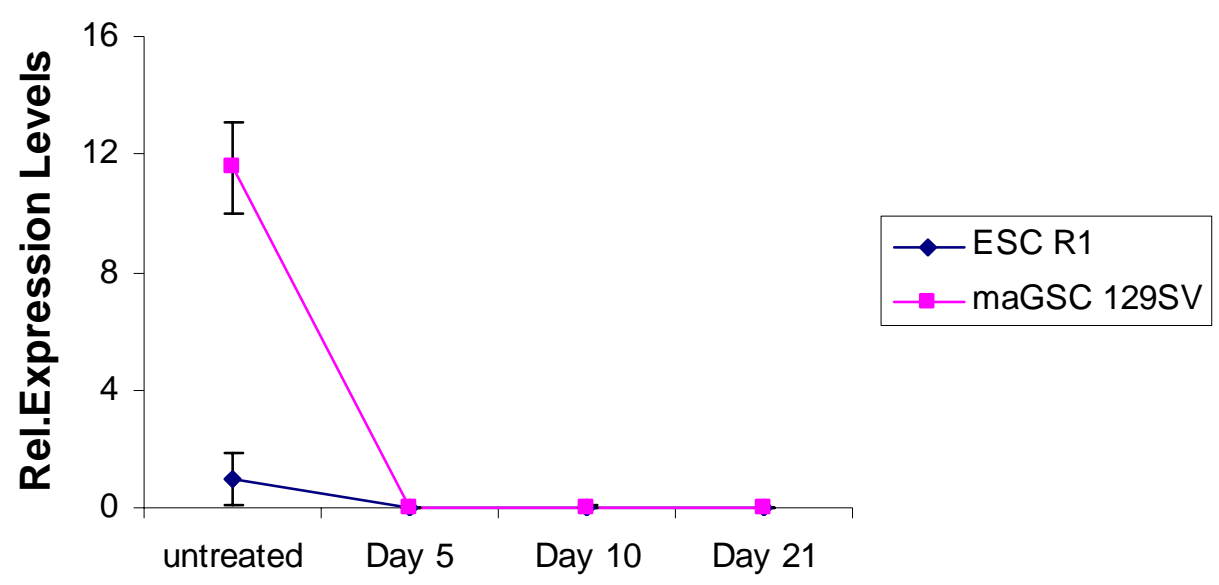

Gel

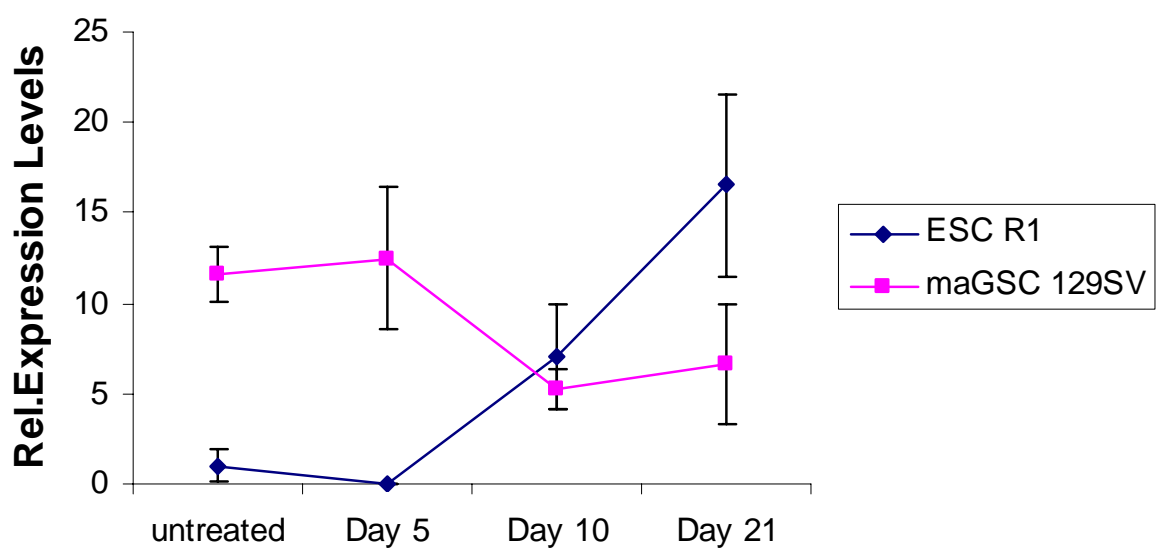

Gel+RA

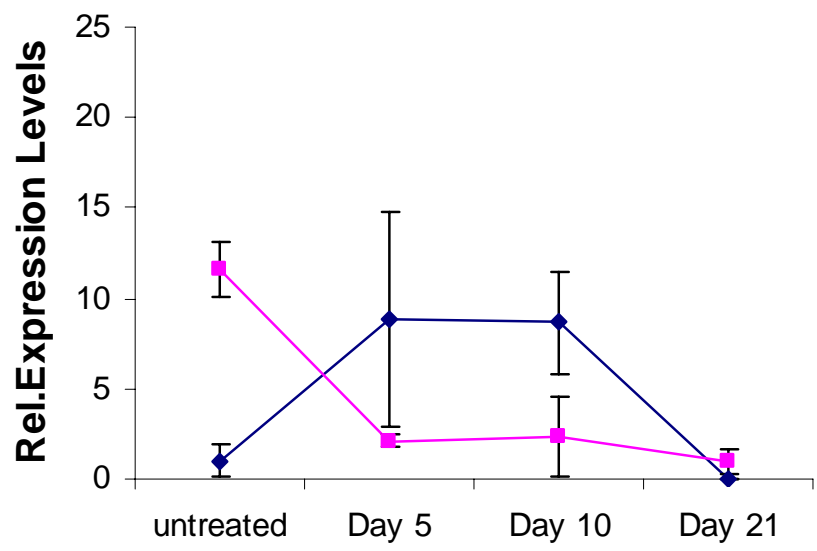

Figure 29. Expression of Dnmt3A mRNA by Real-time PCR in ESCs and maGSCs cultured for 5, 10 and 21 days under all three differentiation conditions compared with untreated cells. Expression levels were normalized and calibrated as in Figure 19. 


\section{LIF+RA}

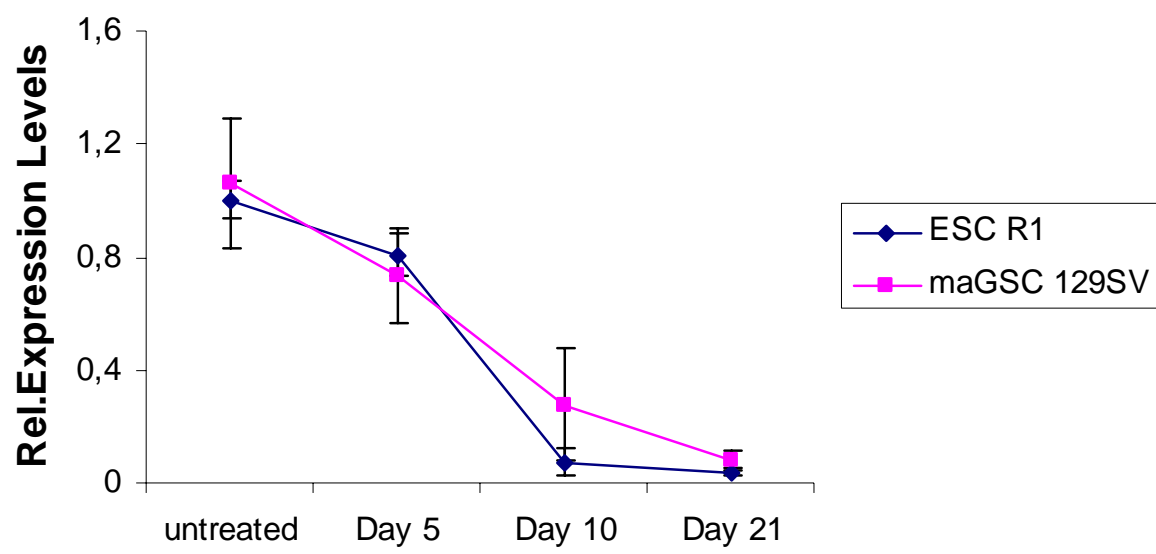

\section{Gel}

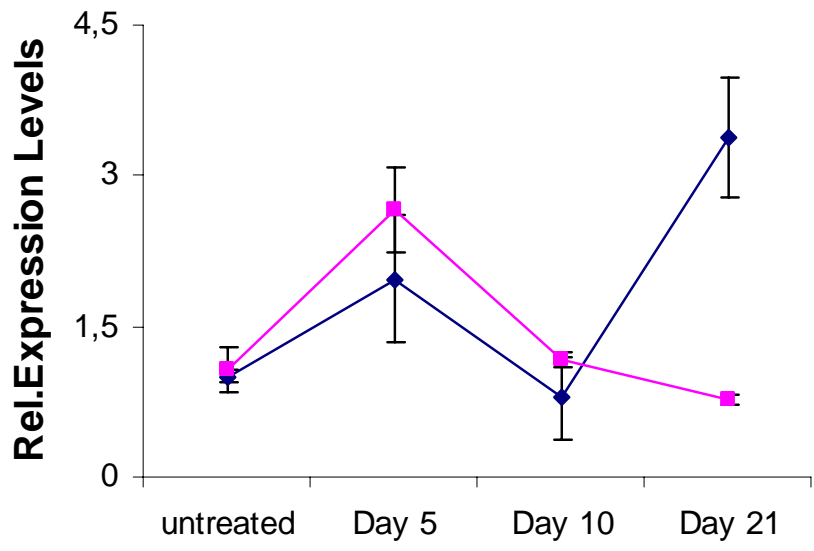

\section{Gel+RA}

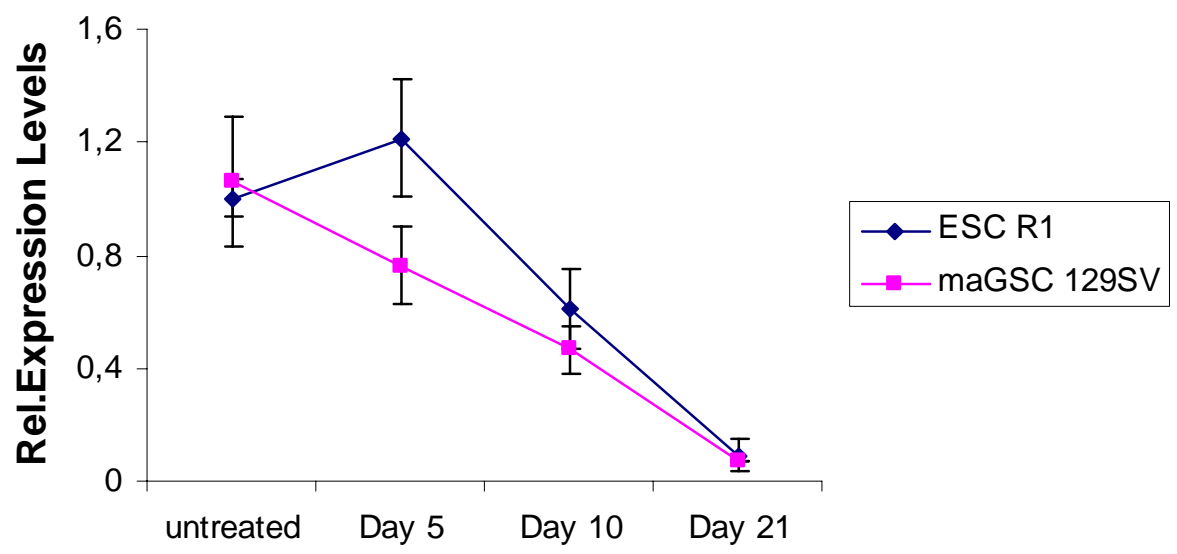

Figure 30. Expression of Dnmt3B mRNA by Real-time PCR in ESCs and maGSCs cultured for 5, 10 and 21 days under all three differentiation conditions compared with untreated cells. Expression levels were normalized and calibrated as in Figure 19. 


\section{Discussion}

Understanding the molecular basis of pluripotency in maGSCs will greatly benefit the field of stem cell biology by providing new pathways and mechanisms that could increase the yield of maGSCs and allow their derivation also in human for regenerative medicine purposes.

Efforts to better integrate the transcriptional and epigenomic networks that control pluripotency in maGSCs are in progress (Meyer 2008; Zechner 2008), and will help to identify the specific mechanisms required for maintenance of pluripotency in maGSCs which until now remain elusive. However, one potential regulatory mechanism that has until now received little attention in ESCs and maGSCs is miRNAs.

Aim of this study was to provide for the first time a comprehensive view of the whole microRNAome of maGSCs compared with that of ESCs. To this end, whole microRNA array expression profiling of maGSCs from two different mouse strains and the respective ESC lines was performed. The array experiments and the subsequent confirmation of the results with qPCR revealed almost an identical miRNA expression profile between ESCs and maGSCs of 129/Sv background, but a distinct miRNA signature of maGSCs derived by the Stra8 transgenic mouse (maGSC Stra8).

Next, this study focused further to all these miRNAs that has been until now annotated a unique role in embryonic stem cell physiology (miR-290 and -302 clusters). To this end, this study compared expression of these miRNAs as well as of protein markers of pluripotency in ESCs and maGSCs under normal and differentiating culture conditions. It was demonstrated by Western blotting that the 
stem cell markers Oct-4 and Sox-2 are present in multiple maGSC lines, as it is known for ESCs. Then, it was demonstrated that miRNAs of the miR-290 and miR-302 family are present in maGSCs, as previously reported for ESCs.

Using standard methods to induce ESC differentiation (e.g., culture in the presence of gelatin and/or retinoic acid), this study showed loss of stem cell markers and miR-290 family expression in ESCs. Likewise, maGSC cultures lost pluripotent marker expression and miR-290 family expression was strongly attenuated, albeit slower than in ESCs. maGSC cultures responded different to differentiation with gelatin than ESCs, resulting in sustained Oct-4 expression and transient miR290 family upregulation. This study also demonstrated that ESC differentiation with gelatin led to a marked and early upregulation of the miR-302 family that was lost over time, which differed with the slow and steady upregulation observed in maGSCs treated similarly.

Consistent with pluripotent marker expression profile, expression levels of most differentiation markers in maGSCs increased later than in ESCs, while both cell types differed as far as expression pattern of de novo DNA methyltransferases is concerned.

Lastly, this study demonstrated miR-290 and miR-302 expression in the pluripotent F9 embryonic carcinoma cell line and correlated their expression under differentiation conditions with stem cell and differentiation markers. 


\subsection{The metazoan microRNAomes and the importance of miRNA-mediated regulation of gene expression}

The existence of very small non-coding RNAs (about $21 \mathrm{nt}$ ) in eukaryotes is already known for many years and was believed to be result of degradation procedures of bigger RNA molecules in cells. Only in the last decade it became obvious that these RNA molecules, called microRNAs, mediate specific cellular processes in cells, playing a key role in cell physiology (Lagos-Quintana et al. 2001; Lagos-Quintana et al. 2002).

Frequently referred to as 'micromanagers of gene expression', miRNAs were first described to regulate developmental timing in C.elegans (Bartel 2004). Since then attempts have been made to elucidate the pathways that govern the biogenesis of these small non coding RNAs. Most miRNAs are located in intergenic regions (like miR-290 and miR-302 families), although some miRNAs are found in the intronic regions of known genes (like oncomirs) (Mendell 2008; miRBase). miRNA genes can be transcribed from their own promoters producing a primary transcript called pri-miRNA, although regulation by the host gene can not be excluded for intergenic located miRNAs. Transcription is mainly mediated by RNA polymerase II and primiRNAs were shown to contain both cap structures and poly(A) tails. In addition, $\sim 50 \%$ of miRNAs are found in close proximity to other miRNAs, and were proved or postulated to be transcribed from a single polycistronic pri-miRNA (miRNA clusters), indicating that these miRNAs are co-ordinately regulated during development (Kim 2005). This is further supported in this study, since expression profile of miRNAs belonging to the same family showed identical expression patterns. 
The widespread presence of miRNAs in metazoan genomes reflects the ubiquitous association of miRNAomes with global gene regulatory networks in cells and numerous publications have revealed their importance in development as well as in disease: miR-430 has been shown to control maternal-zygotic transition in zebrafish. Dicer knockout mice, which means no miRNA biogenesis, die in early embryogenesis (Chen K and Rajewsky 2007; Kanellopoulou et al. 2005). In humans, miRNA disruption has been described in association with several cancers, while miRNAs were also connected with pathogenesis of Fragile X and Di George syndromes (Kim 2005; Mendell 2008). In addition, recently microRNAs targeting Nanog, Oct-4 and Sox-2 in their coding regions have been shown to modulate embryonic stem cell differentiation (Tay et al. 2008). And these are only some examples of experimentally validated miRNA functions.

But why are miRNAs so important for gene expression? Although the importance of transcription as the necessary first step in expression of genes cannot be denied, this does not mean that transcription has always the largest impact on the final concentration of the active gene product. By repressing translation of mRNAs, miRNAs can have a large effect on this concentration, which is the most relevant quantity to the phenotype. 


\subsection{Large scale analysis of maGSCs` microRNAome}

The first works that quantified the genome wide expression of miRNAs in mouse ESCs compared with differentiating cells and adult tissues were those of Chen C et al. (2007) and Strauss et al. (2006) and showed a distinct expression signature of miRNAs in ESCs. Results were similar to those performed in human ESCs by other groups like Suh et al. (2004) and Laurent et al. (2008).

Results presented in this study show that maGSCs derived from SSCs of a 129/Sv background share this unique miRNA signature with ESCs. From all miRNAs tested by global miRNA array expression profiling only two miRNAs (miR-19b and miR-20b) showed differential expression between ESCs and maGSCs in both biological replicates tested. These miRNAs belong to a group of miRNAs called oncomirs. This group consists of miR-17-92 cluster and its paralogues (Fig. 31) and has been annotated a key role in cell proliferation and carcinogenesis (Mendell 2008), and as discussed later also in germ cell development. Although oncomirs do not belong to the same miRNA clusters as ESC-specific miRNAs (miR-290 and miR-302 families), many of the oncomirs are predicted by computational methods to share the same mRNA targets with ESC-specific miRNAs. 


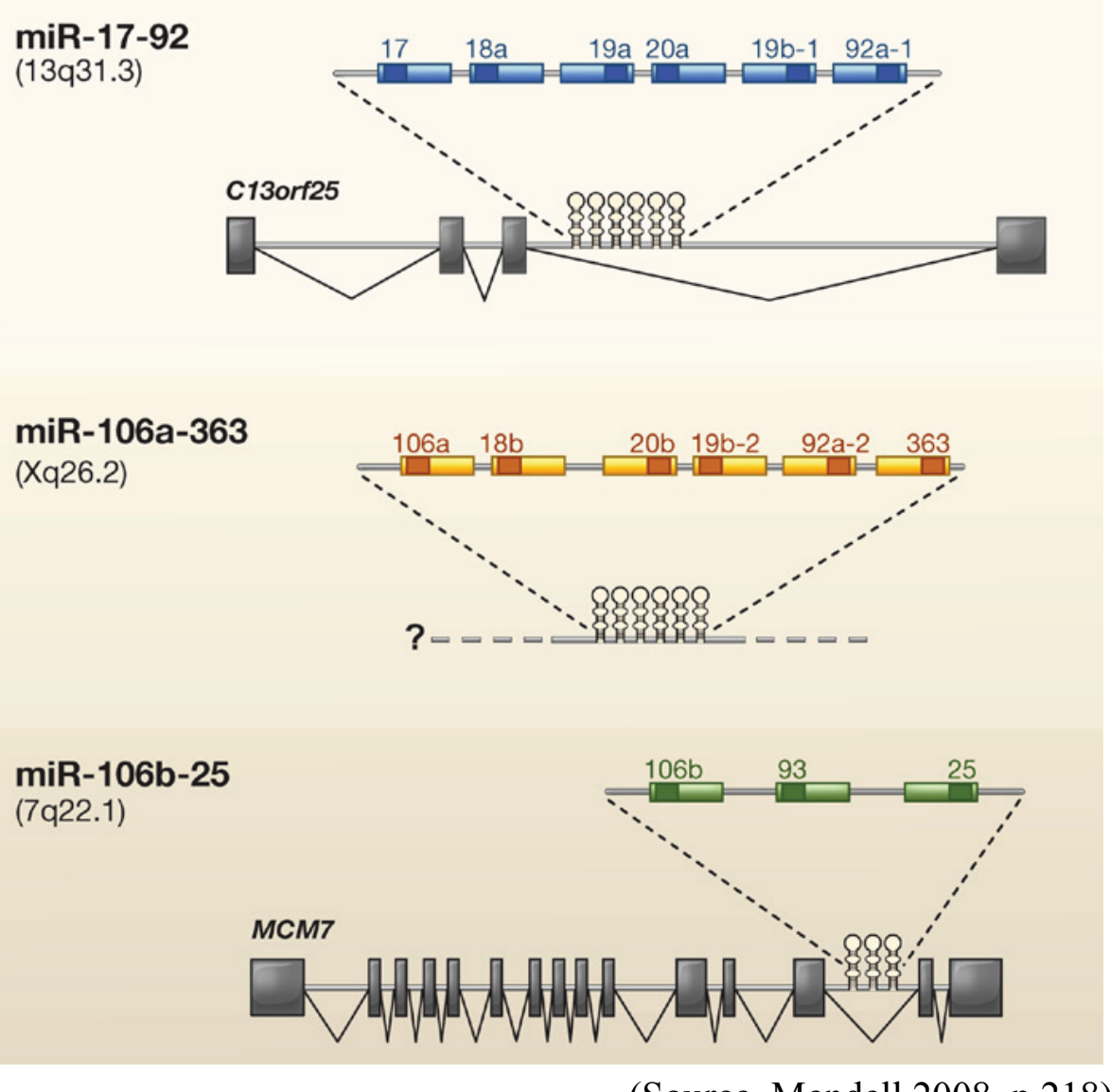

(Source, Mendell 2008, p.218)

Figure 31. Genomic organization of miR-17-92, miR-106a-363 and miR-106b25 miRNA clusters and structures of the respective pri-miRNAs. The last two miRNA clusters are paralogues of the first. miRNAs of all three clusters belong to oncomirs.

However, confirmation of these data with qPCR revealed that these differences between ESCs and maGSCs are restricted to cells originating from a 129/Sv mouse background only.

Thus, it can be concluded that concerning microRNAome, ESCs and maGSCs of $129 / \mathrm{Sv}$ background present almost the same expression signature which is consistent with their similar gene expression and imprinting profile found elsewhere (Meyer 2008; Zechner 2008). Furthermore, most of the observed differences between ESC Stra8 and ESCs and maGSCs from 129/Sv mouse should be attributed to the different genetic background of ESC Stra8, since they were not found to be present in ESCs and maGSCs from the same mouse strain. 
In contrast maGSC Stra8 cells showed a distinct miRNA expression profile from all other cell lines. As discussed later such differences were also confirmed when expression of miR-290 and miR-302 clusters was tested. These results were further correlated with global genome expression profiling and imprinting studies of the same cells. Global genome expression profiling revealed a distinct mRNA expression signature for maGSC Stra8 compared with all other ESC and maGSC lines tested that clustered together. Interestingly, maGSC Stra8 cells expressed germ cell markers like Dppa3 and Stra8 in higher levels than other cell lines. Imprinting studies resulted in similar conclusions.

All these results suggest that maGSCs derived from the transgenic mouse are a distinct pluripotent cell line, compared with all other ESC or maGSC lines. 


\subsection{Expression analysis of ESC-specific miRNAs in maGSCs, ECCs and testis}

In 2003, the Sharp group was the first to identify and describe the ESC specific miR-290 cluster in mouse (Fig. 32) (Houbaviy et al. 2003; Houbaviy et al. 2005), while later the set of ESC specific miRNAs in mouse included also members of the miR-302 cluster (Chen $\mathrm{C}$ et al. 2007; Strauss et al. 2006). These miRNAs were found to be specifically expressed in ESCs and downregulated during differentiation of ESCs into Embryoid Bodies (EBs), while no expression was reported for adult tissues. In addition, these miRNA families have human homologues that have similar genomic organization and expression patterns, suggesting that they may operate as key regulatory networks conserved in mammalian pluripotent stem cells (Suh et al. 2004).

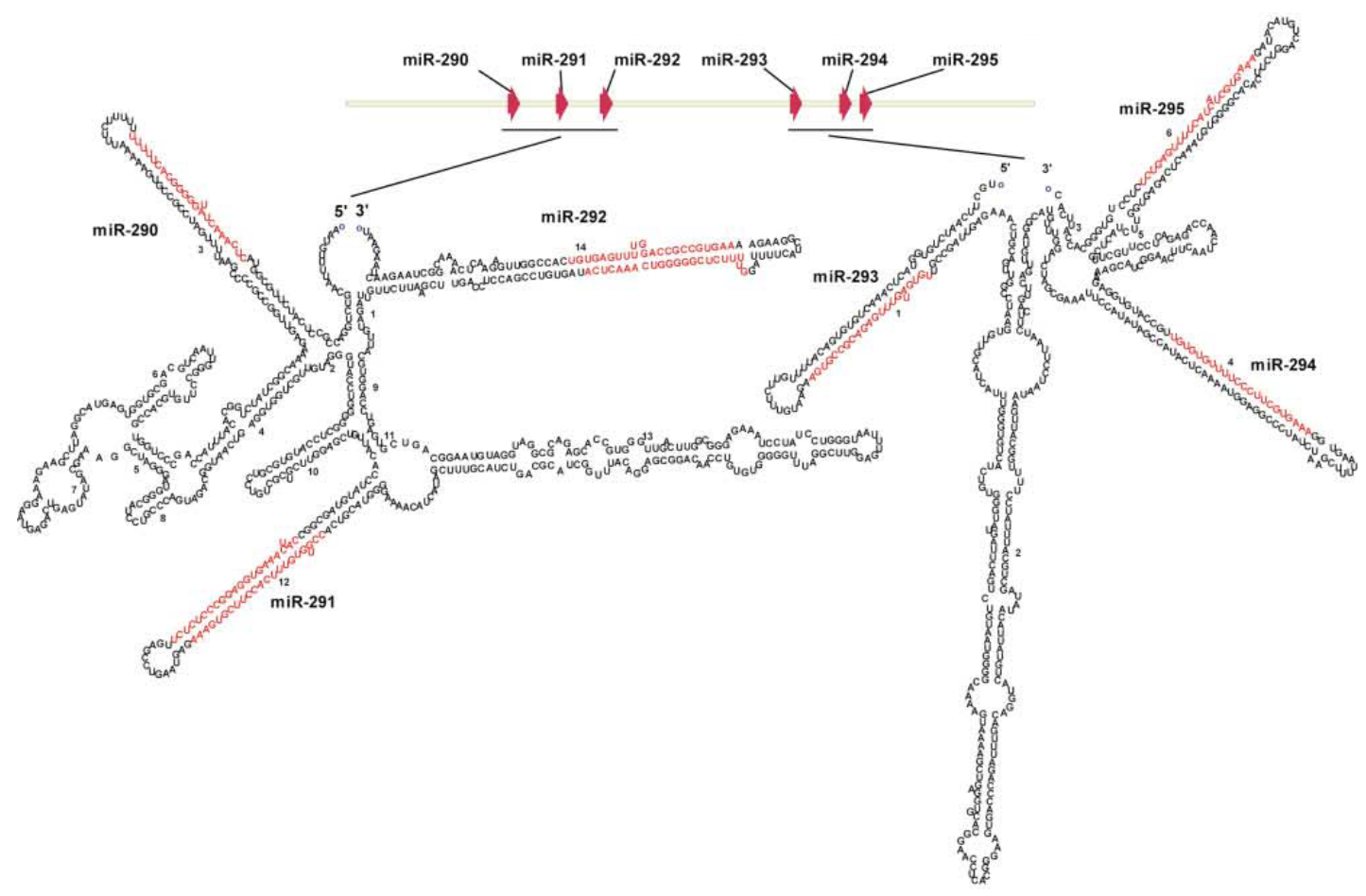

(Source: Houbaviy et al. 2003, p.354)

Figure 32. Genomic organization and structure of miR-290 cluster. Sequences marked in red represent the mature miRNA sequences. 
This study provides evidence that these miRNAs characterize generally pluripotency, since they were detected not only in ESCs but also in all maGSC and ECC lines tested. In contrast, as expected, no expression was detected in non pluripotent cells. Expression of these miRNAs in maGSCs is not restricted to cells of early passage numbers, but remained even after culture for many passages (Fig. 8). However, in contrast to F9 cells, proliferation and expression of these markers in maGSCs is restricted to standard ESC culture conditions, implying that, like in ESCs, expression of these miRNAs is connected with their pluripotent state. This is an important similarity between ESCs and maGSCs that distinguishes them from ECCs and suggests similar underlying mechanisms controlling pluripotency between maGSCs and ESCs.

However, expression levels differed between some maGSCs from different mouse strains, as well as between some ESCs and maGSCs from the same mouse strain. A possible explanation for this, apart from the different genetic background, could be the different passage number of the cell lines tested. As shown in figures of Appendix 4, even under standard ESC culture conditions miRNA expression levels vary between different passage numbers (which for example in case of miR-293 demonstrates an increase in expression levels of more than $50 \%$ in untreated ESCs at day 21, Figure c of Appendix 4A, compared with untreated ESCs at day 5, Figure a of Appendix 4A).

For this reason in all other experiments, cell lines of the same passage number and the same mouse strain $(129 / \mathrm{Sv})$ were used to eliminate this effect. In this case, when cell lines of the same passage number are used, maGSCs 129SV seem to express these miRNAs in similar or slightly lower levels than ESCs from the same background (ESC R1).

Finally, the great heterogeneity reported by previous groups in ESC cultures (Houbaviy et al. 2003), which is due to stochastic or difficult 
to control factors (like serum batch, medium age, frequency of subculture etc), should always be taken into account when trying to explain the differences observed in gene and microRNA expression. In this study, these factors were eliminated to the most possible extent by two ways: Firstly, by the use of biological duplicates where possible and applicable. Secondly by constant evaluation of the pluripotent state of the cells tested, to exclude that differences in miRNA levels should be attributed to improper culture conditions or spontaneous differentiation of the cells.

The fact that quantification of these miRNAs in testis revealed no relative expression as compared with ESCs and maGSCs does not exclude the existence of these miRNAs in SSCs in adult testis. In fact a recent work (Hayashi et al. 2008) reported that miRNA 290 cluster together with miR-17-92 cluster are highly expressed in PGCs and spermatogonia and that miRNA biogenesis is essential for their development, and even in the current study these miRNAs were detectable with RT-PCR in adult testis (data not shown). Similarly, no Oct-4 was detected in the testis sample although previous reports demonstrate Oct-4 immunoreactivity by histochemical methods from embryonic through adult testes (He $\mathrm{Z}$ et al. 2007). These results could be attributed to the fact that spermatogonial stem cells constitute only $0.03 \%$ of all germ cells (Tegelenbosch and de Rooji 1993) making it difficult to detect Oct-4 by Western analysis. For this reason, compared with maGSCs, the normalized Real-time PCR data for miRNAs from the whole testis would be also practically zero. However, whether these miRNAs really exist in adult SSCs, and which functional implications this has for their differentiation potential remains to be elucidated. 


\subsection{Expression profile during differentiation}

A second major goal of this work was to examine the effects of different factors that are commonly used for in vitro differentiation of pluripotent cells on the expression of members of ESC-specific miRNA families. Since the great importance of ESCs and maGSCs lies on their regenerative potential in medicine, understanding the underlying mechanisms of their differentiation in vitro, requires again a substantial knowledge about their transcriptional, epigenetic and miRNA expression profile during this process.

Three general approaches have been employed to initiate in vitro differentiation of pluripotent cells (Keller 2005). With the first method cells are allowed to aggregate and form three dimensional colonies (embryoid bodies). In the second, cells are cultured directly on stromal cells, and in the third cells differentiate in a monolayer (Nishikawa et al. 1998). This last approach can minimize the influence of neighboring cells and supportive stromal cells to the results and in this regard it was used as one of the simplest methods in this study.

In addition, due to the big number of differentiation strategies so far described in the literature, this study concentrated on the most important factors that prevent or induce differentiation in ESC culture, namely LIF and RA. It is well known that the signaling pathway mediated by LIF (LIF-STAT3) is, together with that mediated by Oct4 , critical for self-renewal of mESCs and maintenance of feeder-free ESC lines (Liu et al. 2007; Rao 2004; Kurosawa 2007). Furthermore, RA has been found to negatively regulate Oct-4 and to induce differentiation of ESCs and ECCs (Rohwedel et al. 1999; Tighe and Gudas 2004). 


\subsubsection{Members of miR-290 family are connected with maintenance of pluripotency}

The observation of other authors that the members of miRNA family 290 are downregulated in ESCs during differentiation is supported by this study and a downregulation was found to be realized during maGSCs differentiation. However, in maGSCs under Gel and Gel+RA condition, miRNA levels remain high for a longer period than in ESCs or even increase transiently (Gel condition). These differences seem to correlate with the differences in the differentiation status of these cells. Under Gel condition, Nestin and Vimentin are upregulated earlier in ESCs, while Oct-4 expression decreases later in maGSCs. In addition, FL+LIF+RA, which is the only condition in maGSCs at day 10 with a significant increase in most differentiation markers, is characterized by a strong downregulation of miRNA levels.

Since the expression profile of Oct- 4 corresponds to that of members of miRNA family 290 in both ESCs and maGSCs, our results indicate that expression of these miRNAs is more connected with maintenance of pluripotency than with differentiation.

\subsubsection{Members of miR-302 family are induced during first stages of in vitro differentiation}

Chen $C$ et al. (2007) have studied expression of members of the miRNA 302 family in ESCs at days 3, 6 and 9 during embryoid body formation in absence of LIF. They found that these miRNAs are negatively correlated to differentiation time. This expression profile in ESCs is different from that it was obtained in this study, since during the first five days of differentiation under Gel condition, all members of miRNA family 302 are strongly upregulated. Transient 
upregulation of these miRNAs in ESCs is also observed in the presence of RA, although not so strongly as in Gel condition.

Expression profiles of these miRNAs in maGSCs demonstrate similarities and differences compared with ESCs. Under Gel condition, strong upregulation of miRNA levels is also observed, and addition of RA was found to result also in an upregulation of these miRNAs. However, in maGSCs upregulation under Gel condition takes place slowly. At day 21 miRNA levels in maGSCs depict 10-30 fold increase, while in ESCs 20-100 fold increase is reached already at day 5. This gradual increase in maGSCs correlates to the differences in differentiation status between ESCs and maGSCs mentioned above. In addition, the increase under Gel+RA condition in maGSCs occurs at day 5 and not at day 10 as in ESCs.

Since the expression profile of Oct-4 does not correspond to that of 302 miR-family members, our results suggest that these miRNAs may be more connected with response of pluripotent cells to differentiation than with the undifferentiated state itself.

This is in contradiction to the observation that, even in undifferentiated cells, miRNAs 302 are present but can be explained by the observation that cultures of pluripotent cells contain spontaneously differentiated cells (Houbaviy et al. 2003). It is not yet clear the reason why the group of $\mathrm{C}$. Chen did not observe this transient upregulation of miR-302 family during differentiation.

\subsubsection{Further proofs about connection of these miRNAs with pluripotency or differentiation}

The connection of members of miRNA family 290 with pluripotency and that of members of miRNA family 302 with the process of 
differentiation is further supported by miRNA analysis in ECCs and comparison at day 10 of Gel+RA condition with Gel+RA from day 5 .

In ECCs, where addition of RA is followed by an increase in Nestin and Hnf-4 expression, miR-302 family is upregulated. Similarly, when Gel+RA and Gel+RA from day 5 conditions are compared, high levels of miR-290 family are connected with low levels of miR-302 family and most of differentiation markers (with the exception of Hnf-4) and vice versa.

This connection is also supported by the findings of Tang et al. (2007). They have shown that the miRNA 290 family belongs to the most significant miRNAs strongly upregulated in early mouse embryogenesis from two-cell stage onwards. This is exactly the stage when Oct-4 expression increases (Schoeler et al. 1989). In contrast, miRNA family 302 does not show significant expression changes.

\subsection{4 miRNAs and mesoderm formation}

An interesting finding of this work is that the extreme upregulation of miR-302 family under Gel condition is connected with a predominantly mesoderm character of these cells (only under this condition, expression of Brachyury was observed). Differentiation to mesoderm requires Bmp-4 signaling and serum used in the ESC medium is known to be able to induce similar activities. However, at the moment it remains unclear whether these miRNAs have a positive or a negative effect on this process. It is possible that these miRNAs target an inhibitor of this pathway resulting in increased Brachyury expression. However, since after day 5 both levels of Brachyury and miRNAs 302 decrease dramatically, it can not be excluded that this decrease is due to these miRNAs, that may target an important pathway mediator. This is further supported by the observation of 
Alisch et al. (2007) that reported ectopic expression of Brachyury in mouse embryos with impaired Argonaute 2 function, which is essential for miRNA mediated translational silencing.

In fact, computational approaches that predict possible mRNA targets for different miRNAs (TARGETSCAN software) revealed that miRNAs of miR-302 family (and miR-290 family also) can target both activators and inhibitors of the Bmp-4 pathway (Table 4). This applies also to other pathways (Wnt pathway, Nodal signaling) that like Bmp-4 are involved in mesoderm formation and germ cell development (for a comprehensive review about all the above mentioned pathways see Tam and Loebel 2007).

\begin{tabular}{|c|c|c|c|}
\hline mRNA target & Pathway & Action & $\begin{array}{l}\text { Targeting } \\
\text { miRNAs }\end{array}$ \\
\hline Tob1 & Bmp-4 & Inhibitor & $292 \_3 p$ \\
\hline Tob2 & Bmp-4 & Inhibitor & $\begin{array}{l}\text { 294, 295, 302, } \\
\text { 291_3p }\end{array}$ \\
\hline $\begin{array}{ll}\text { Lefty } 1 \text { and } \\
\text { Lefty2 }\end{array}$ & INodal & Inhibitor & $\begin{array}{l}\text { 294, 295, 302, } \\
291 \_3 p\end{array}$ \\
\hline Dkk1 & Wnt & Inhibitor & $\begin{array}{l}\text { 294, 295, 302, } \\
291 \_3 p\end{array}$ \\
\hline Smad 1 & Bmp-4 & Activator & $291 \_5 p$ \\
\hline Smad 2 & Nodal & Activator & $\begin{array}{l}\text { 294, 295, 302, } \\
291 \_3 p\end{array}$ \\
\hline Smad 5 & Bmp-4 & Activator & $2915 p$ \\
\hline
\end{tabular}

Table 4. Computational prediction of targeting miRNAs for genes mediating Bmp-4, Wnt and Nodal pathways. Prediction was performed using the TARGETSCAN software available on line. Only miRNAs belonging to miR290 or miR-302 families and with binding sites at 3' UTR of these genes that were conserved in both human and mouse are presented. 
For these reason, functional studies that would overexpress these miRNAs are necessary for elucidation of their roles in these processes.

\subsection{5 miRNAs and germ cell development}

Another interesting finding of this in vitro differentiation study is some differences -compared with ESCs- in the expression profile of maGSCs regarding pluripotency markers and miRNAs. In particular, maGSCs proved to be more resistant in loss of pluripotency upon deprivation of LIF than ESCs. The same pattern was realized for miRNAs of 290 family, while in contrast, miR-302 family (which from this study was shown to correlate with beginning of differentiation) is upregulated later in these cells. This profile is in agreement with the profile mentioned earlier for PGCs, where cells were mainly enriched in miR-290 family and in the oncomirs, but not in miR-302 (Hayashi et al. 2008). Thus, it can not be excluded that maGSCs under Gel condition represent an equivalent cell type of PGCs.

To test this, our group has further evaluated expression of Dppa3, which is the most representative PGC marker (Lacham-Kaplan 2004), in the same differentiating cells employed in this study (A. Pantazi, unpublished work). The results (Fig. 33) confirm this hypothesis, since maGSCs under Gel condition express Dppa3 in significantly higher levels than ESCs. Taking into account that Bmp-4 pathway, that may be regulated by miR-302 family, is necessary for PGC development and spermatogenesis (Itman and Loveland 2008), possible functional implications of this family in germ cell development should be tested in the future. 


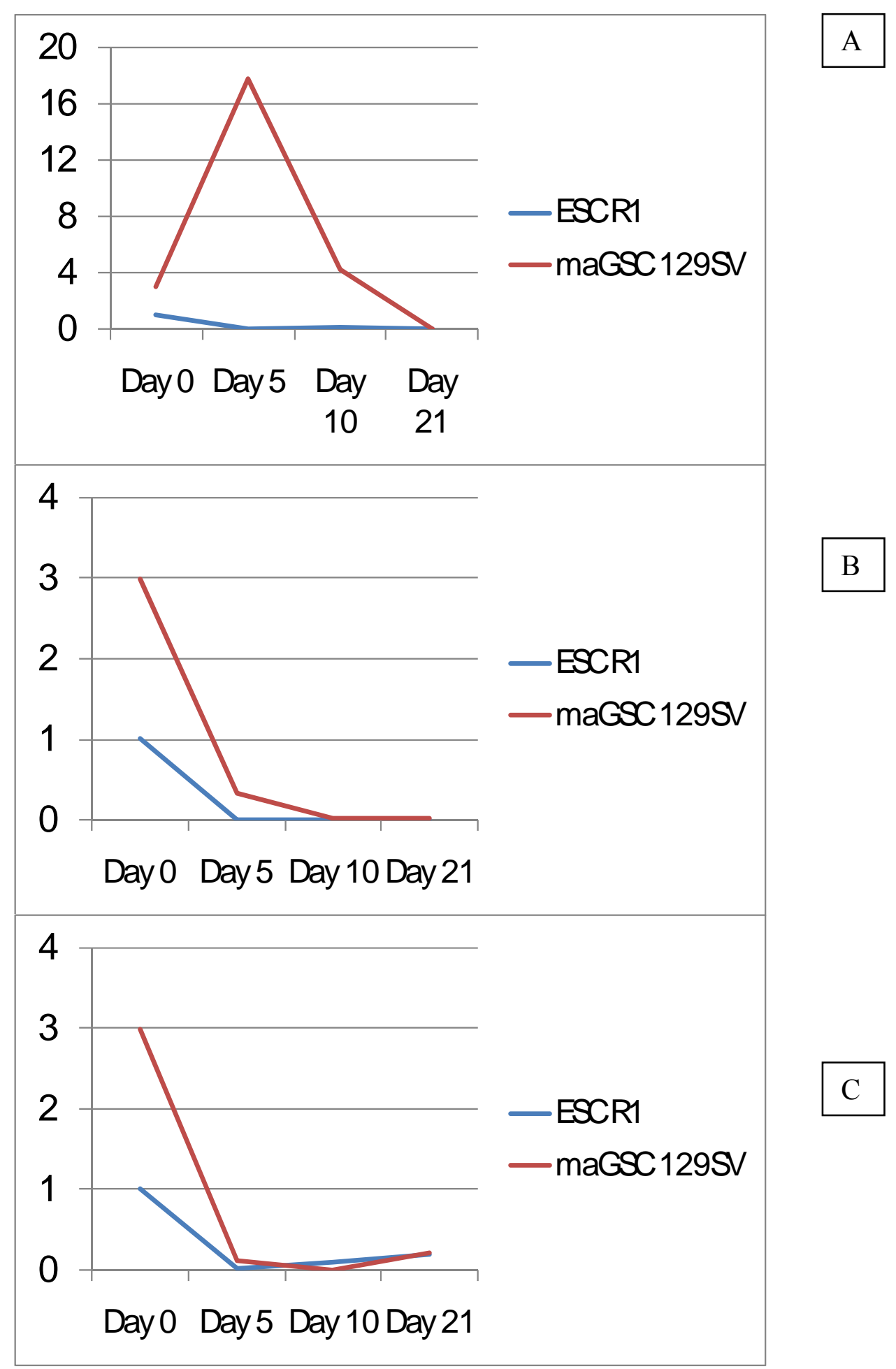

(Kindly provided by Mrs A.Pantazi, Institute of Human Genetics, Goettingen)

Figure 33. Dppa-3 mRNA expression in ESC R1 and maGSC 129SV during differentiation. A: Gel, B: Gel+RA, C: FL+LIF+RA. Dppa 3 is predominantly expressed in undifferentiated maGSCs and in maGSCs under Gel condition compared with ESCs. Results are presented as normalized relative expression levels. ESC R1 at day 0 was used as calibrator. 


\section{5 miRNAs and de novo DNA methylation in maGSCs}

Until now, little is known about the exact function and role of these miRNAs in pluripotency and development. Most studies have achieved a global miRNA "knock out" by targeting Dicer which is indispensable for miRNA maturation. These studies revealed that Dicer KO mice embryos are lethal and Dicer-KO ESCs presented differentiation defects (Kanellopoulou et al. 2005). The reason for this was partially explained by the results of a recent study that showed that miRNAs of miR-290 family control de novo DNA methylation by targeting Rbl2, which is an inhibitor of Dnmt3A and Dnmt3B transcription (Sinkkonen et al. 2008). These methyltransferases are responsible for de novo DNA methylation during differentiation of pluripotent cells.

Since maGSCs presented differences in the expression profile of these miRNAs compared with ESCs it was challenging to test them for expression of DNMT3A and B in the differentiation model employed in this study. Our results revealed that the different miRNA expression profile of maGSCs corresponds to a different expression pattern for DNMT3A. In light of recent findings showing a significant decrease in global DNA methylation of maGSCs during differentiation compared with ESCs (Zechner 2008), these results support further the hypothesis that differentiating maGSCs are an equivalent type of PGCs, which during differentiation show a similar global DNA demethylation (Fig. 34). It is reasonable to suggest that this decrease in DNA methylation in maGSCs could be attributed to the low levels of DNMT3A in these cells during differentiation. Since Sinkkonen et al. (2008) performed their experiments mainly in Dicer KO cells, additional studies that will specifically downregulate these miRNAs in 
pluripotent cells are needed to solidify also the relationship between these miRNAs and de novo DNA methylation in maGSCs.

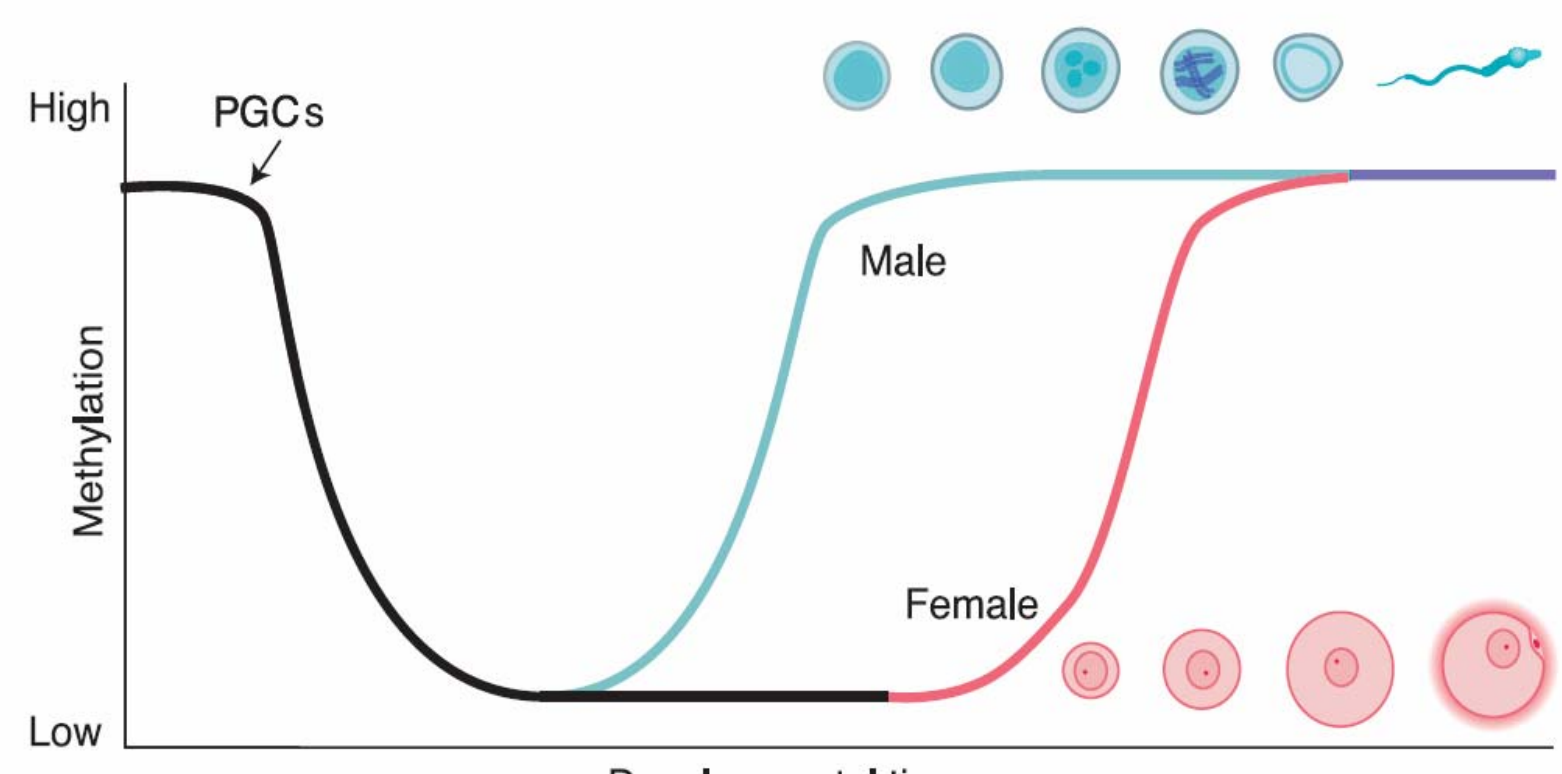

Developmental time

(Source: Reik et al. 2001, p.1090)

Figure 34. Global DNA methylation profile of PGCs during development towards male or female gametes. PGCs show a transient global loss of methylation prior to differentiation into germ stem cells. 


\subsection{Conclusions and Perspectives}

Derivation of pluripotent cells and their differentiation into specific tissues belong to the foundations of regenerative medicine. However, the available sources of pluripotent cells until recently, were either unsuitable for this use (like ECCs) or faced ethical and other practical problems like immunorejection (like ESCs). The recent derivation of iPS cells is still facing problems like the low yield of cells, the increased carcinogenic potential as well as problems arising from their derivation with use of virus vectors. For these reasons isolation for the first time of pluripotent cells from an adult tissue like maGSCs, without any genetic selection or induction should be regarded as a revolution in this field.

However, very little is known until now about the mechanisms that may control pluripotency in maGSCs. Only recently was possible to derive similar cells also from human adult testis (Conrad et al.2008). However, these cells differ in many aspects form human ESCs and mouse maGSCs, especially in that it was not able to produce a clonal cell line from them. Since this characteristic is common in all other pluripotent cell types, future attempts should focus on elucidation of the nature of these cells isolated from the human adult testis.

This work revealed in maGSCs a miRNA profile almost identical to that of ESCs and thus it solidifies the relationship between maGSCs and other pluripotent stem cells (i.e., ESCs, F9 ECCs) in rodents.

In addition, it implies that maGSCs possess some unique characteristics concerning the way expression levels of pluripotency related miRNAs respond to differentiation. These characteristics may be connected with the PGC like gene expression profile that these cells present during differentiation and their relative resistance to 
induction to differentiation. It remains to be elucidated in future studies whether this applies also to the in vitro derivatives of PGCs, the EGCs.

Finally, this work lays the foundations for future functional studies concerning the role of miRNAs of miR-290, miR-302 and oncomirs' families in mesoderm formation and germ cell differentiation. The questions that have arisen need to be addressed by studies involving overexpression and downregulation of these miRNAs in vitro, as well as in vivo. 


\section{Summary}

Spermatogonial stem cells (SSCs) isolated from the adult mouse testis and cultured have been shown to respond to culture conditions and become pluripotent, so called multipotent adult germline stem cells (maGSCs). Recently a new group of non coding RNAs, called microRNAs (miRNAs), has been implicated to play a key role in regulation of embryonic development and pluripotency in mouse embryonic stem cells (ESCs). The objective of the current study was to test similarities and differences between maGSCs and ESCs with respect to miRNAs. Whole miRNA array expression profiling showed that despite slight differences in expression of some oncomirs, undifferentiated maGSCs and ESCs originating from 129/Sv mouse strain are equivalent cell types from the viewpoint of microRNAome. In contrast, undifferentiated maGSCs originating from the transgenic Stra8-EGFP-Rosa mouse are a distinct cell type regarding miRNAs. miRNAs belonging to the 290 and 302 clusters have been previously classified as ESC-specific. This study shows that these miRNAs generally characterize pluripotent cells. They are expressed not only in undifferentiated ESCs but also in undifferentiated maGSCs as well as in the F9 embryonic carcinoma cell (ECC) line. In addition, this study tested the time-dependent influence of different factors that promote loss of pluripotency on levels of these miRNAs in all three pluripotent cell types. Using standard methods to induce ESC differentiation, this study showed loss of stem cell markers and miR-290 family expression in ESCs. Likewise, maGSC cultures lost pluripotent marker expression and miR-290 family expression was strongly attenuated, albeit slower than in ESCs. In addition, ESC differentiation with gelatin led to a marked and early upregulation of the miR-302 family that was lost over time, while a slow and steady upregulation was observed in maGSCs treated similarly. Differences in time and extent of differentiation observed between ESCs and 
maGSCs correspond to similar differences in the expression profiles of both miRNA families, suggesting similar underlying mechanisms in maintenance of pluripotency between the two cell types. These results indicate that the 290-miRNA family is connected with Oct-4 and maintenance of the pluripotent state. In contrast, members of the 302-miRNA family are induced during first stages of in vitro differentiation in all cell types tested. Therefore, detection of miRNAs of miR-302 family in pluripotent cells could be attributed to the proportion of spontaneously differentiating cells in cultures of pluripotent cells. Furthermore, differences in the miRNA levels observed between ESCs and maGSCs correlate with differences observed in expression of de novo DNA methyltransferases and global methylation state in these cells. All these results are consistent with ESC-like nature of maGSCs and their potential as an alternative source of pluripotent cells from non-embryonic tissues. At the same time, however, they imply that the mechanisms that control differentiation in pluripotent cells may differ in some aspects between ESCs and maGSCs, and miRNAs may be responsible for these differences. 


\section{Appendices}

\section{Appendix 1}

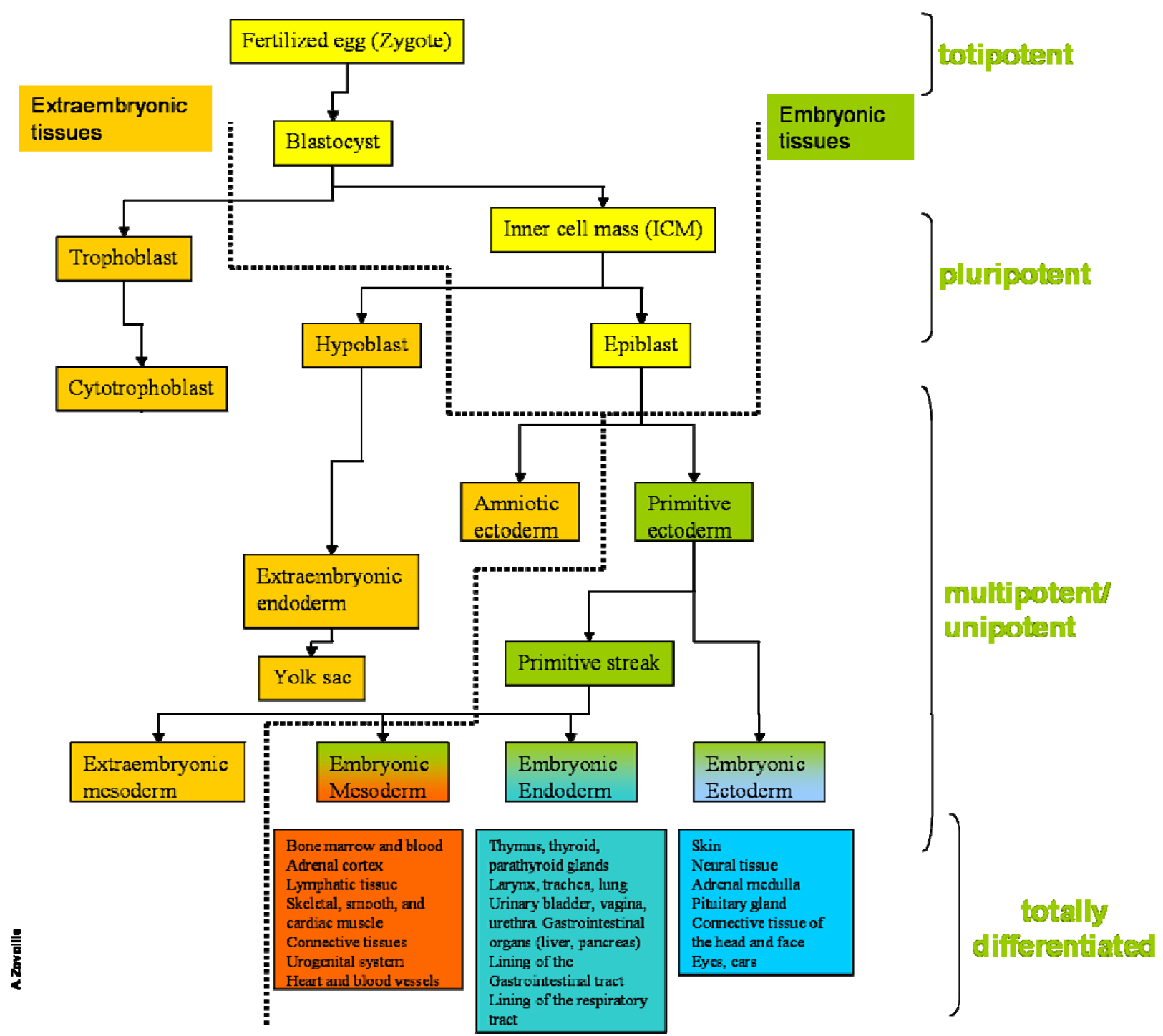

Figure 1. Overview of early stages of mouse embryonic development and differentiation potential of cells from the three germ layers. The point lines and the different colours separate embryonic from extra embryonic tissues. The arrows connect each developmental stage with the previous stage from which it originates. The legends on the right define the differentiation potential of the cells, while the colour boxes at the bottom present examples of tissues orginating from each of the three germ layers. 

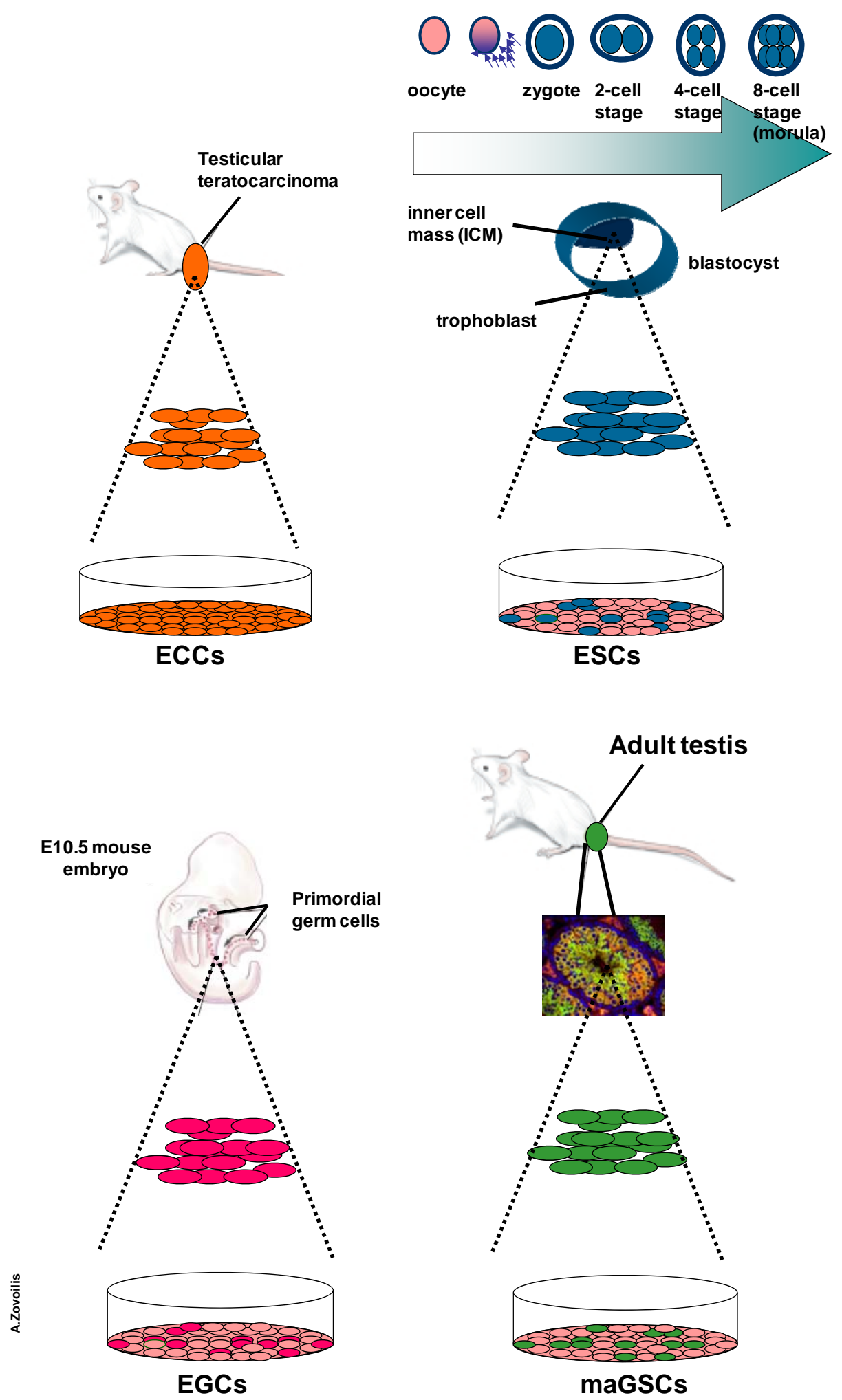

Figure 2. Sources of different types of pluripotent cells. Each subfigure represent a different pluripotent cell type in vitro with its respective origin cell population in vivo. 


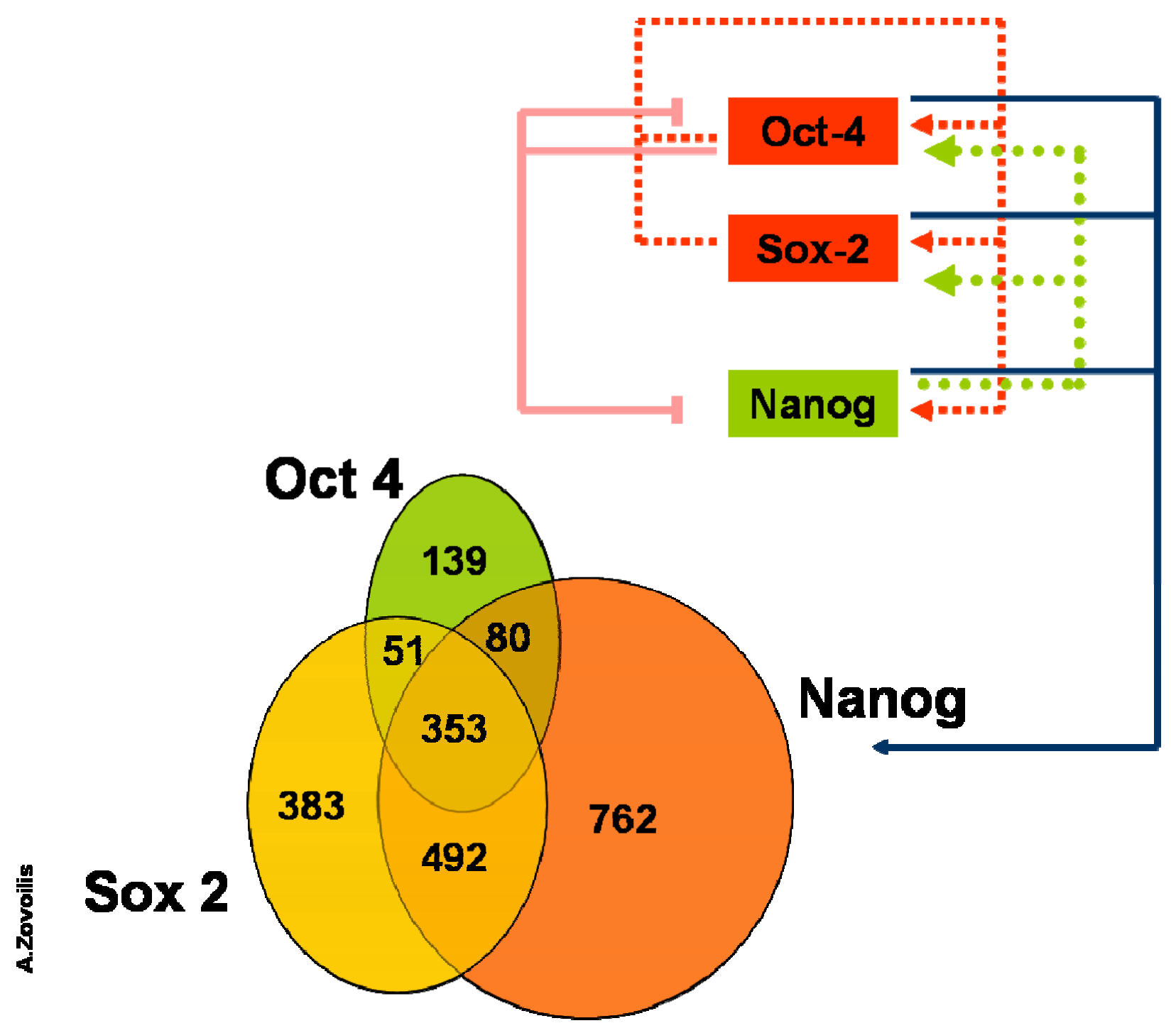

Figure 3. Transcription factors controlling pluripotency in mouse ESCs. The core of ESC transcriptional network consists of three transcription factors (Oct4, Nanog and Sox-2) that interact with each other and control expression of hundreds of genes. Arrows depict a positive effect through transcriptional activation. Blind arrows depict a negative effect through transcriptional inhibition. Circles include known target genes for each transcriptional factor. Overlapping regions show genes regulated in common from these factors either synergistically or independently (modified by Pan and Thomson 2007 and Rodda et al. 2005). 


\section{Appendix 2}

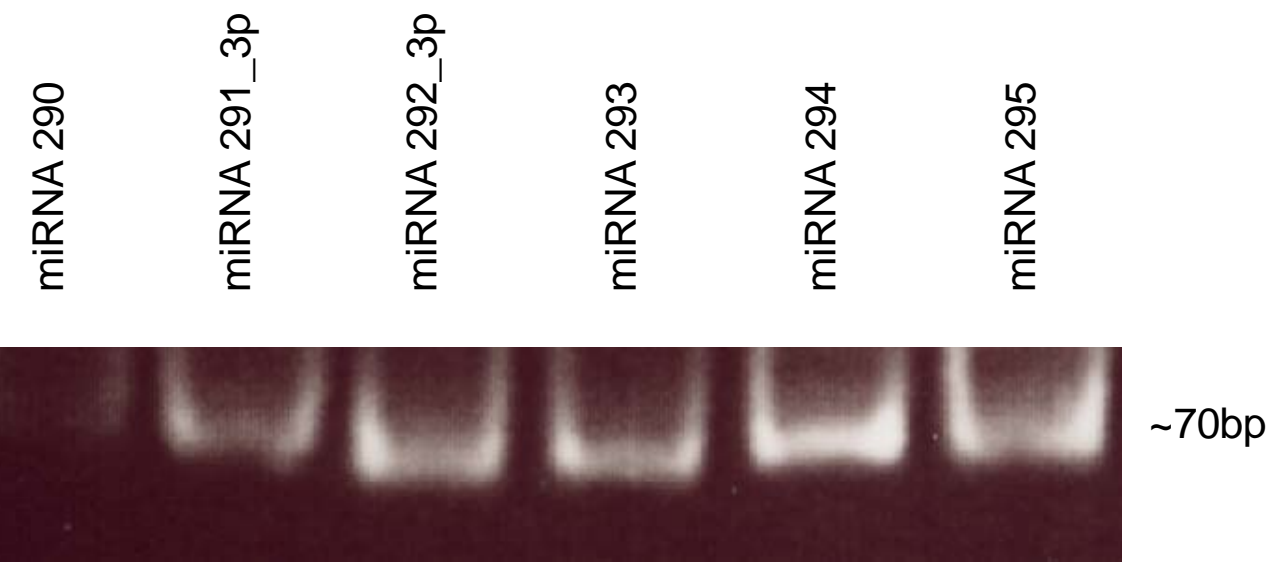

Figure 1. Example of PAGE electrophoresis of the PCR amplicons for members of miR-290 family in ESC R1 cells. These cells were further used as positive controls for construction of standard curves in qPCR. Similar were the results for miR-302a, $b$ and $d$. 


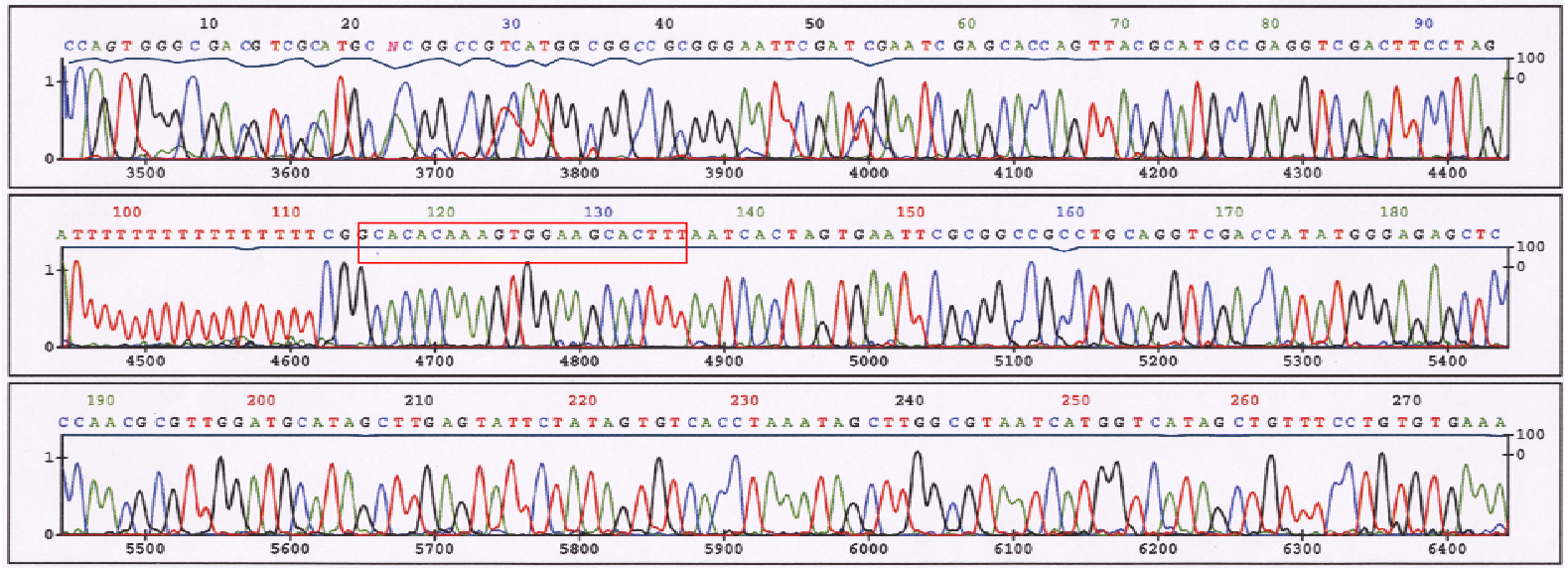

\section{Alignment of Query to mature miRNAs}

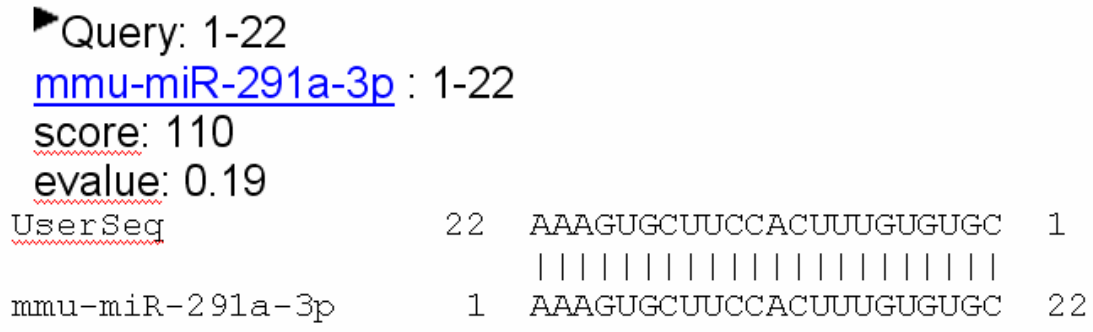

Figure 2. Example of sequence analysis of the cloned PCR amplicons for members of miR-290 and 302 family. In the figure the sequence for miR291_3p amplicon is presented. The presented alignment below the sequence is the result of comparison of the amplicon sequence with all mature miRNA sequences in Sanger miRNA database. Amplicon sequence marked in red corresponds to the mature miR-291_3p sequence in Sanger database, confirming the specificity of the PCR product. Sequence analysis confirmed the specificity of PCR products of all other miRNAs tested except miR-302c, as well as of Vimentin, Nestin, Brachyury, Hnf-4, Nkx-2.5, Oct-4, Dnmt3a, Dnmt3b and Sdha. 


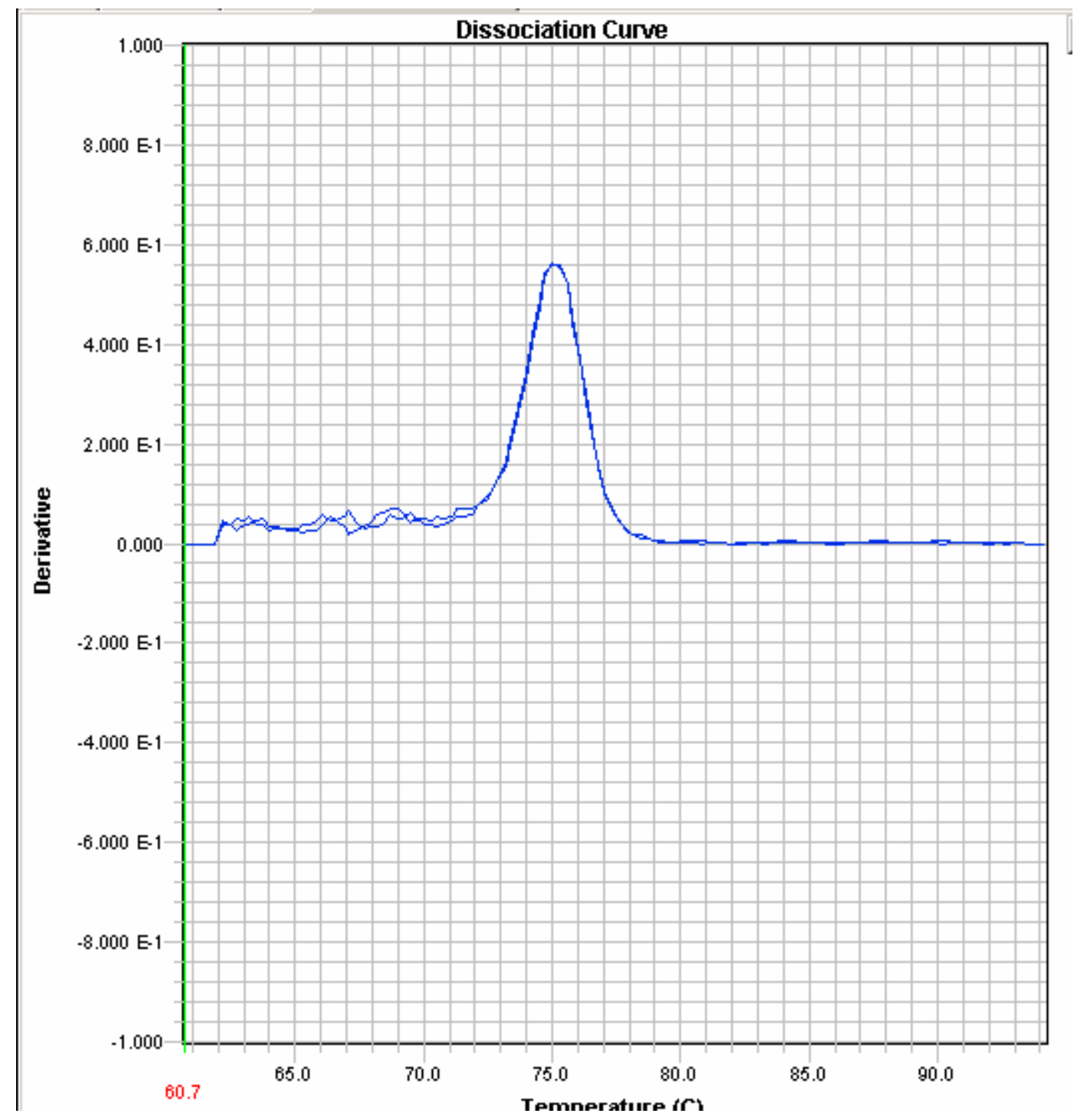

Figure 3. Specificity and reliability of qPCR results was tested with the respective dissociation curves. The existence of only one peak in all runs confirmed the specificity of the PCR results. In this example dissociation curve of the PCR for miR-292_3p in ESC R1 cells is presented. Similar curves were obtained for all other miRNA and mRNA genes tested except miR-302c were the analysis showed multiple peaks. 

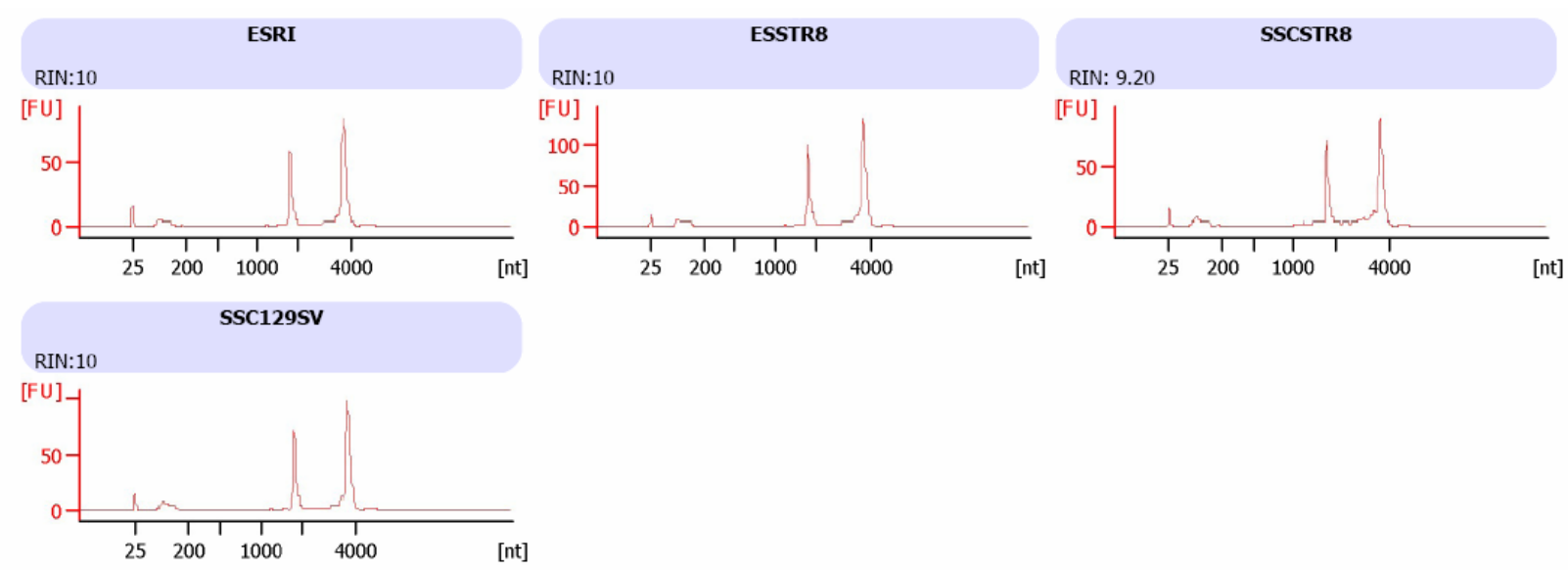

Figure 4. Example of test of RNA integrity (with the respective RIN values) for RNA used in miRNA array experiments. Acceptable are RIN values at least above 7. All samples tested in this study fulfilled this requirement (ESRI=ESC R1, ESSTR8=ESC STRA8, SSCSTR8=maGSC STRA8, SSC129SV=maGSC 129SV). 


\section{Appendix 3}

A. Expression profiles of members of the miRNA 290 family in ESCs and maGSCs (Results, Section 3.4.3.). In this figure the different conditions are superimposed to facilitate comparison.
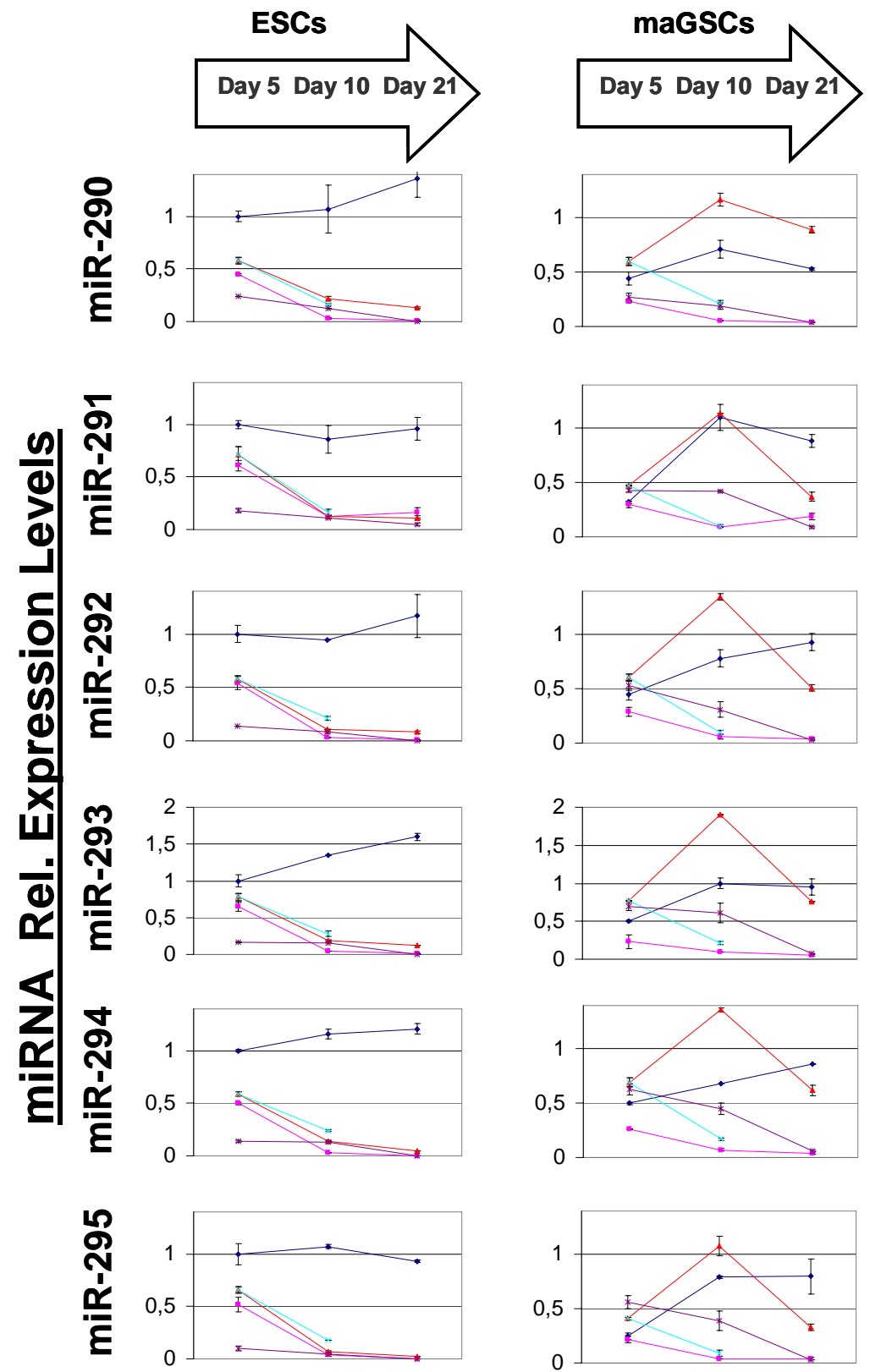
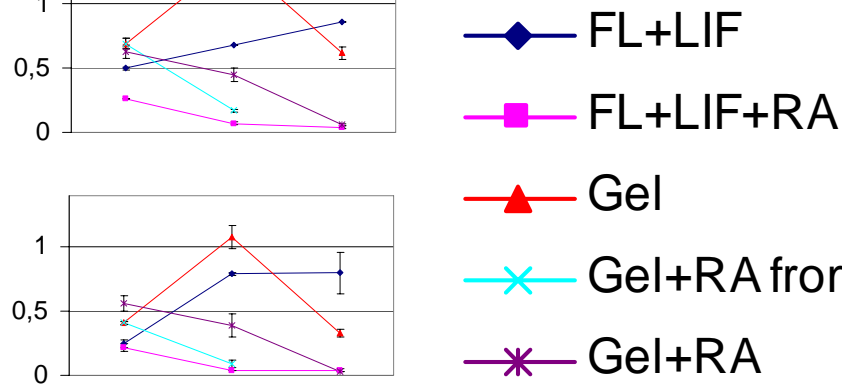

Gel

$x-$ Gel+RA from D5

$\rightarrow$ Gel+RA 
B. Expression profiles of members of the miRNA 302 family in ESCs and maGSCs (Results, Section 3.4.4.). In this figure the different conditions are superimposed to facilitate comparison. The upper part depicts results in ESCs, the lower part in maGSCs. Because of the strong upregulation of miRNA levels under Gel condition as compared to other culture conditions the results are given separately (left column).
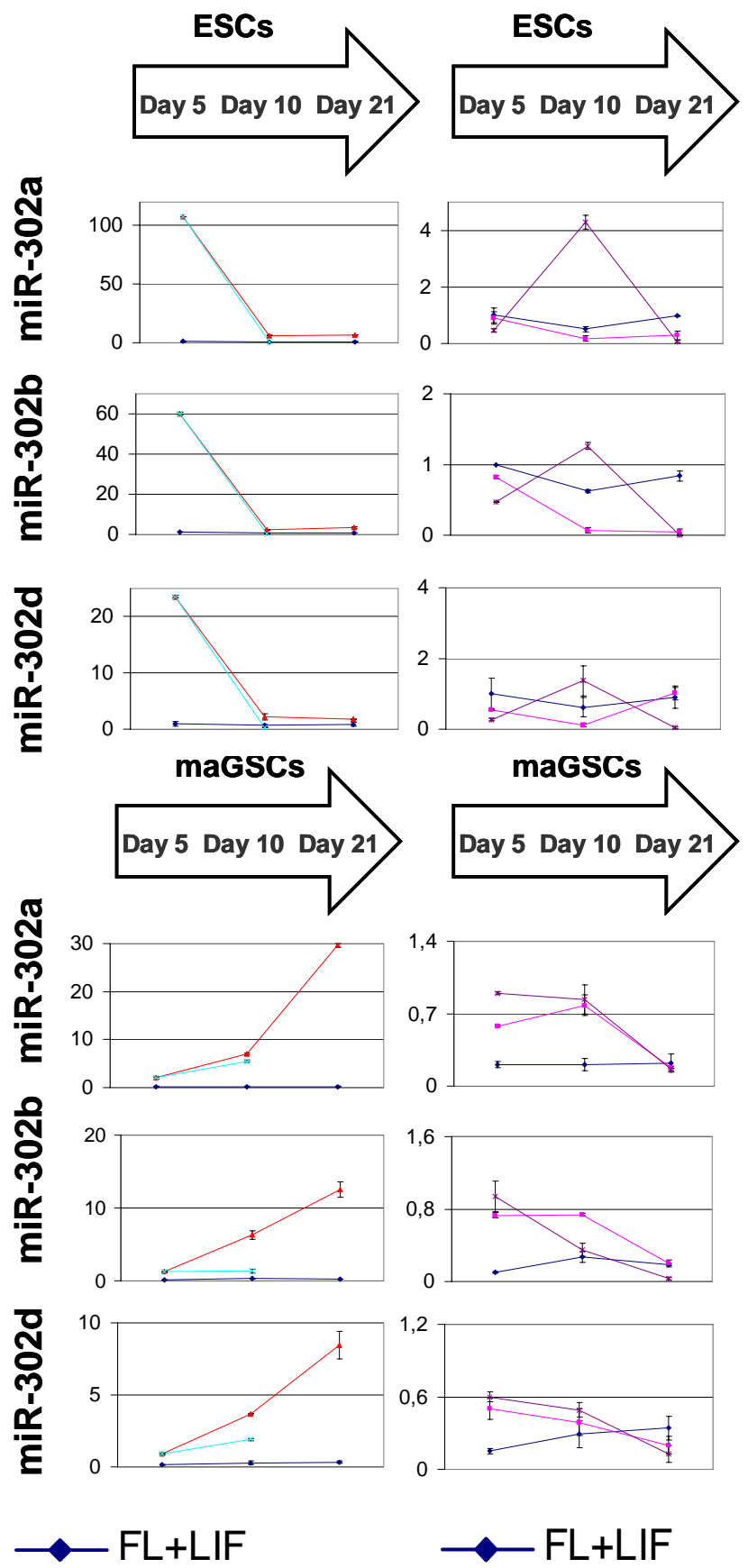

$\longrightarrow$ Gel

$\rightarrow-F L+L I F+R A$

$\times$ Gel+RA from D5

$\rightarrow-$ Gel+RA 


\section{Appendix 4}

A. Expression profiles of members of the miRNA 290 family in ESCs and maGSCs from mouse strain 129/Sv (ESC R1 and maGSC 129SV) under different culture conditions after 5 , 10 and 21 days (Subfigures a, b and c respectively) in culture. a, b, c and depicted in each subfigure above the different conditions indicate statistical significance for the following pair comparisons, a: comparison of each differentiation condition with untreated cells, b: comparison between FL+LIF+RA and GEL, c: comparison between FL+LIF+RA and GEL+RA, d: comparison between GEL and GEL+RA. Combination of two or three letters indicates statistical significance for more than one comparisons. For example, a b d above GEL condition refers to comparison of this condition with all other conditions. The letters apply to all miRNAs of each condition with the exception of these miRNAs with an \# above them, which indicates no statistical significance for the corresponding miRNA and the corresponding comparison. For example \#a above miRNA-290 in one condition means no statistical significance for miR-290 in this condition compared with untreated cells. The line that cuts the diagram in two parts, separates ESCs (left) from maGSCs (right). All levels were normalised to endogenous control (RNU6B) and calibrated to the value of untreated ESC R1 $(\mathrm{FL}+\mathrm{LIF})$ at day 5 (subfigure a).

B. Expression profiles of members of the miRNA 302 family in ESCs and maGSCs from mouse strain 129/Sv (ESC R1 and maGSC 129SV) under different culture conditions after 5, 10 and 21 days in culture (Subfigures $\mathrm{a}, \mathrm{b}$ and $\mathrm{c}$ respectively). The line that cuts the diagram in two parts, separates ESCs (left) from maGSCs (right). All levels were normalised to endogenous control (RNU6B) and calibrated to the value of untreated ESC R1 (FL+LIF) at day 5 (Fig.a). For symbols indicating statistical significance, see Figure A. Additionally, asterisk above miR-302a in subfigure $b$ indicates statistical significance only for this miRNA for the comparison of the respective condition with untreated cells. 
A

a miR-290 amiR-291 amiR-292 口miR-293 amiR-294 口miR-295

a

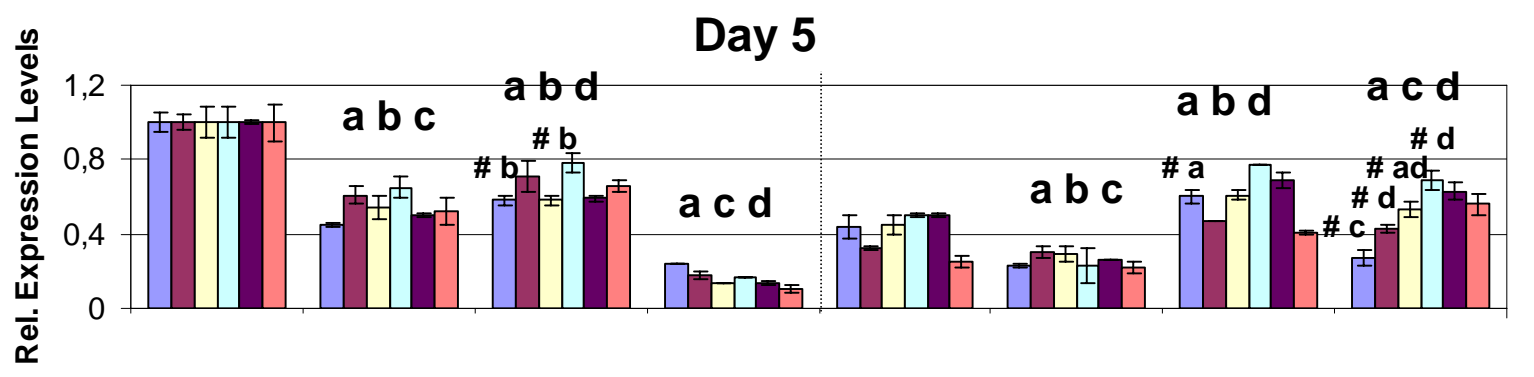

b

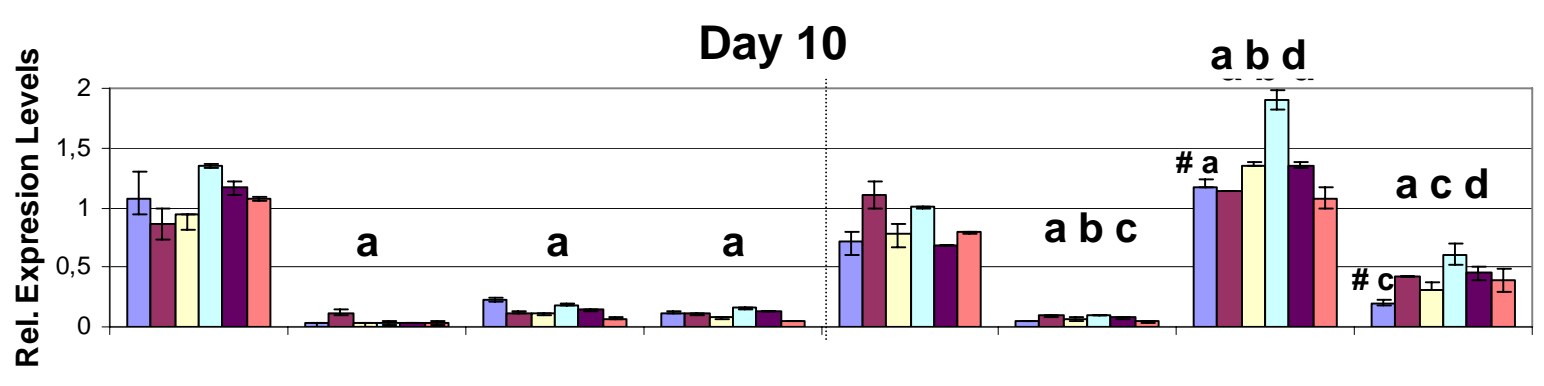

C

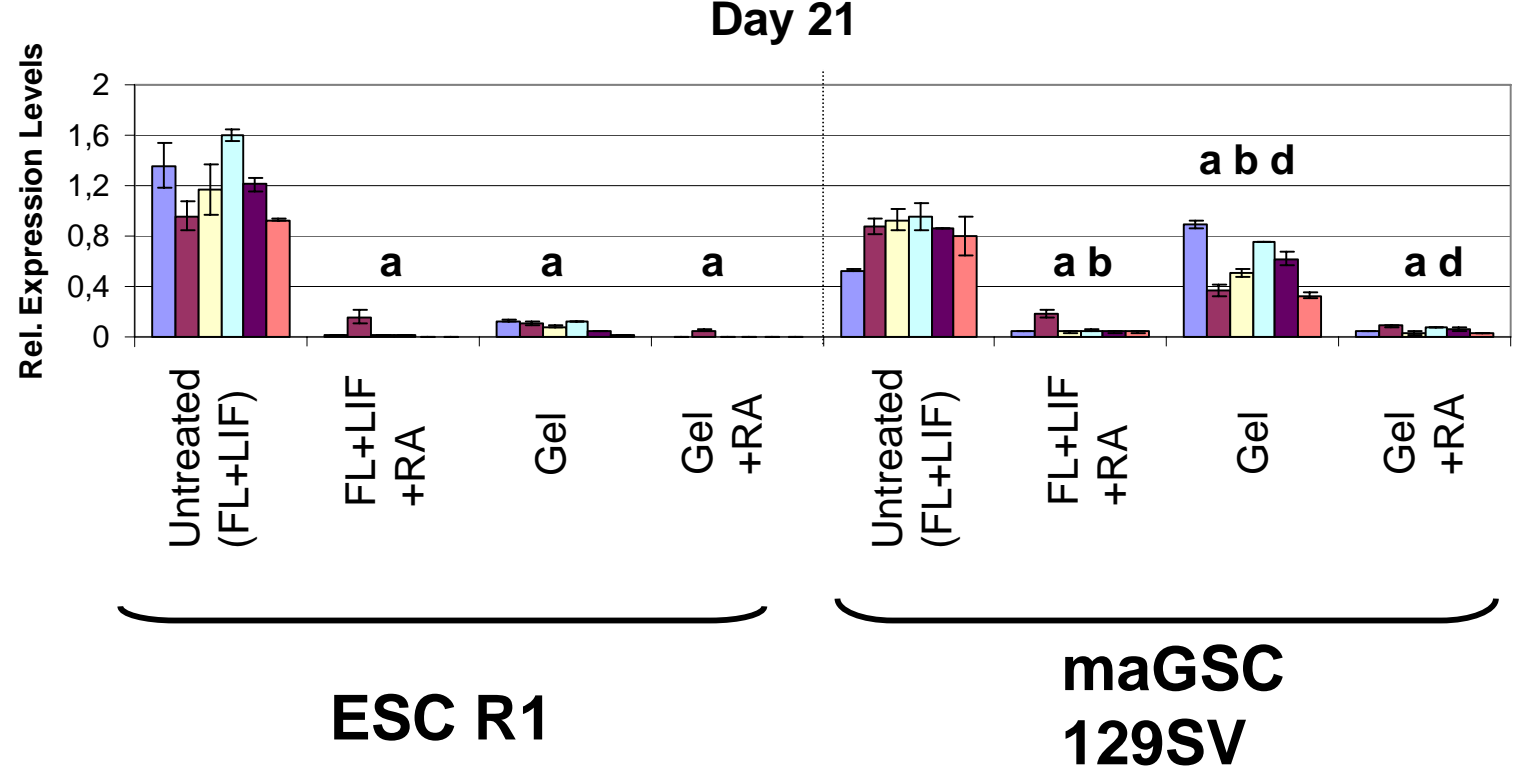


B

a

$\square \mathrm{miR}-302 \mathrm{a} \quad \square \mathrm{miR}-302 \mathrm{~b} \quad \square \mathrm{miR}-302 \mathrm{~d}$

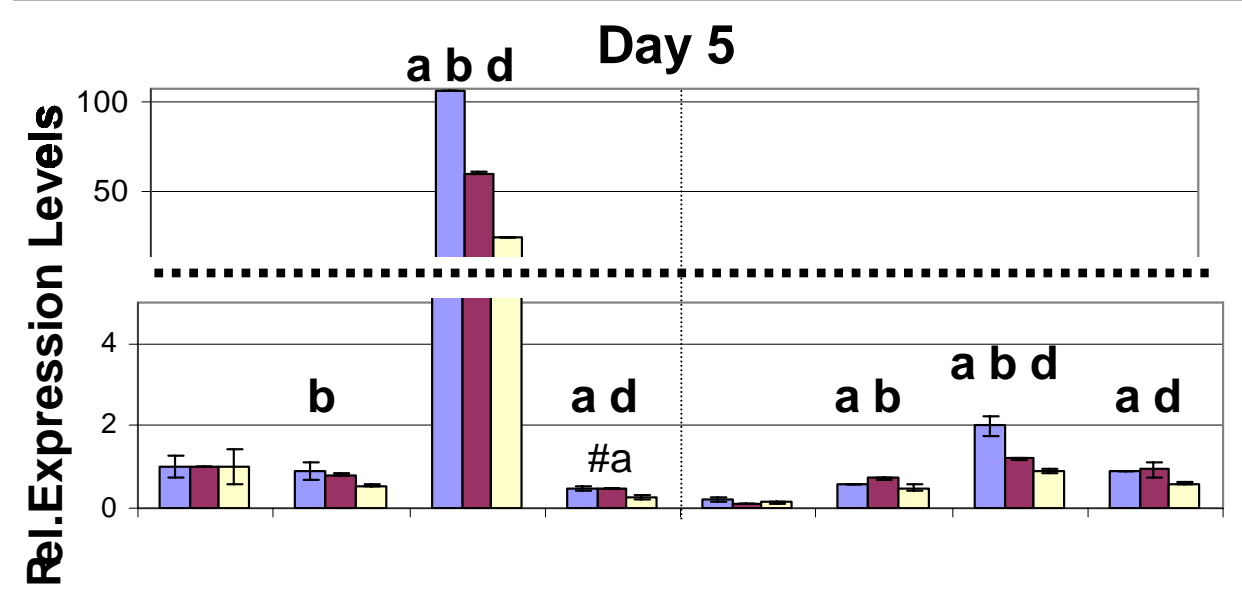

b

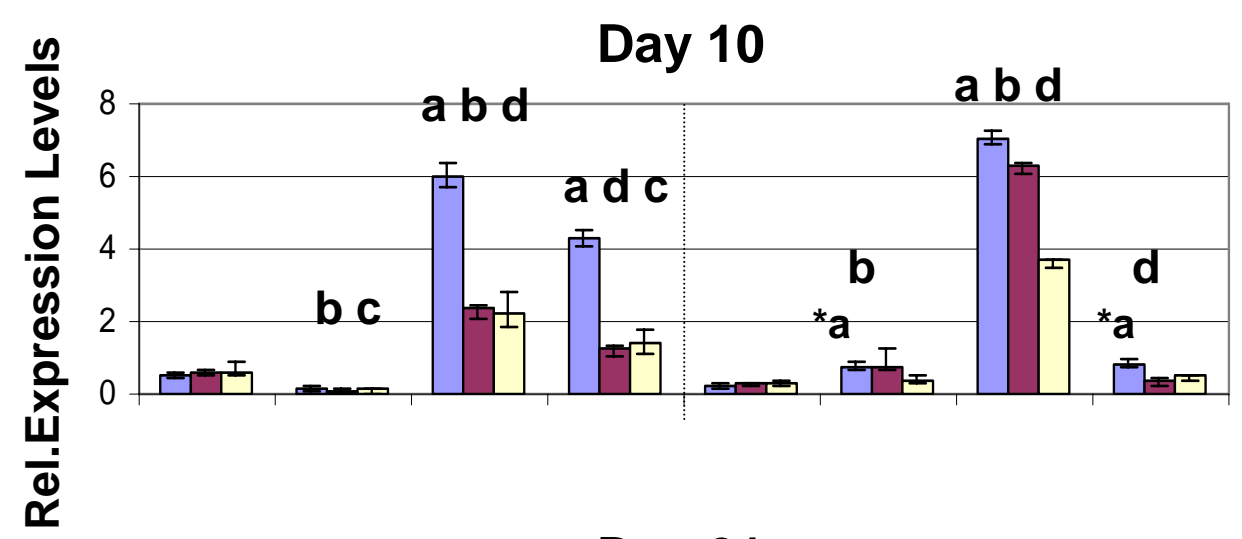

Day 21

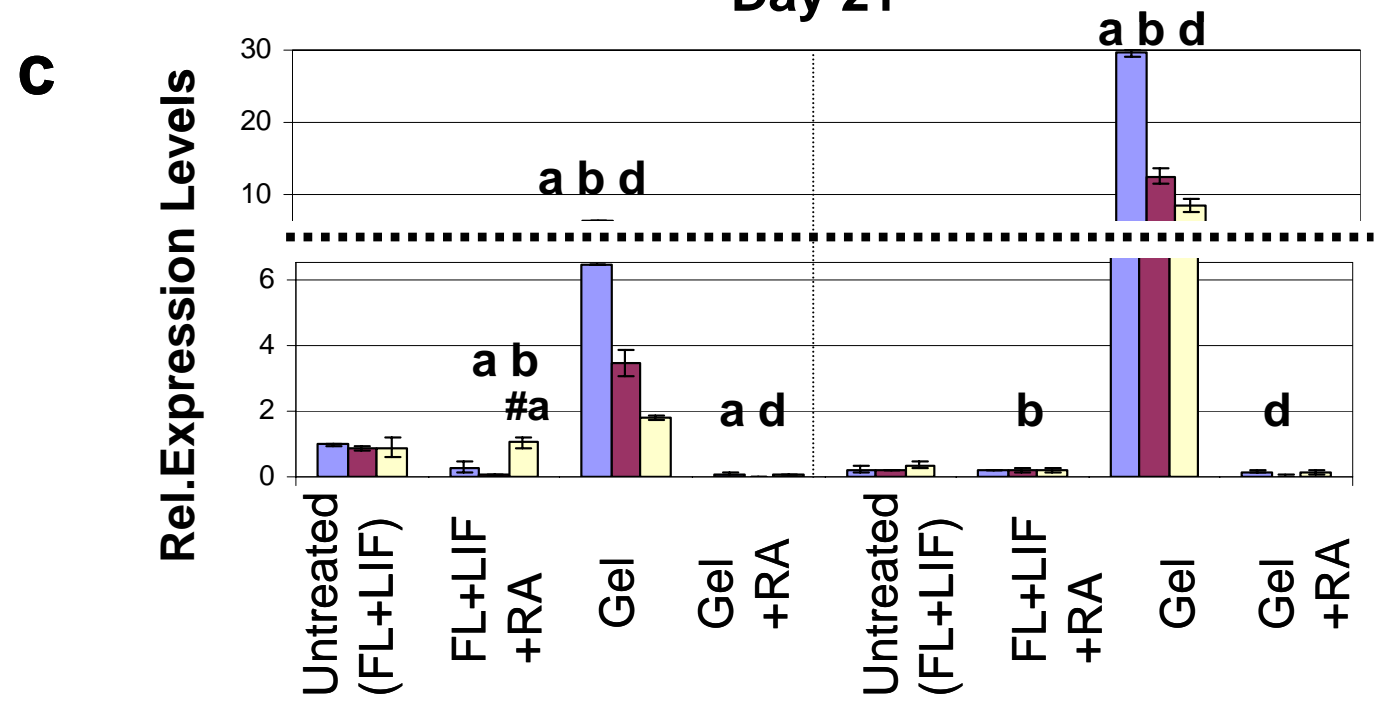

ESC R1

MaGSC

129SV 


\section{Appendix 5}

Expression profiles of differentiation markers (Vimentin, Nestin, Hnf-4 and Nkx-2.5) in ESCs and maGSCs from mouse strain 129/Sv (ESC R1 and maGSC 129SV) under different culture conditions after 5,10 and 21 days in culture (vertical lines separate differentiation conditions of the same day from those of other days). Asterisks indicate statistical significance for the comparison with untreated cells. All levels were normalised to endogenous control (Sdha) and calibrated to the value of untreated ESC R1 (ESC R1 FL+LIF at day 5). (a) Expression profile in differentiating ESC R1 cells. (b) Expression profile in differentiating maGSC 129SV cells.

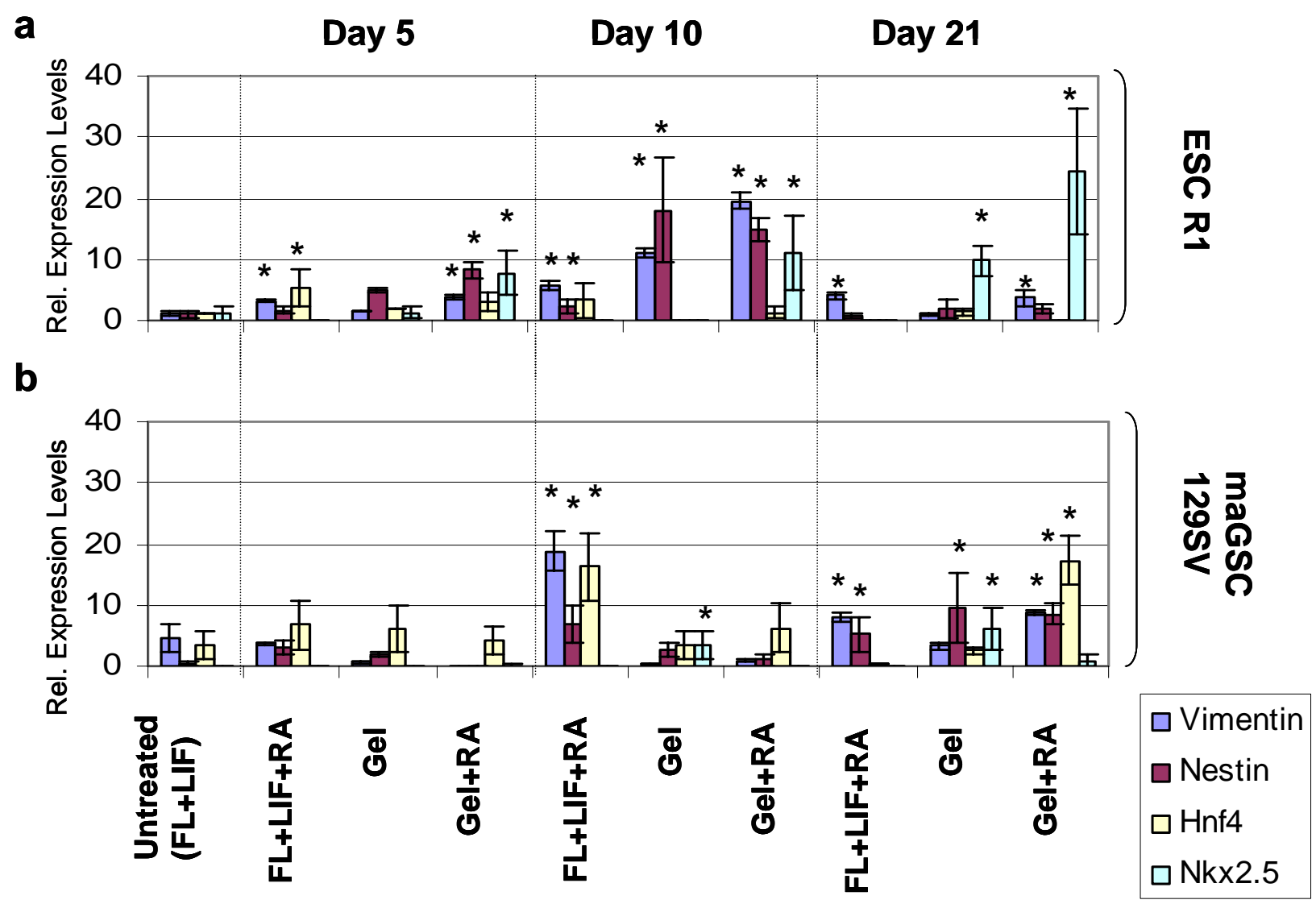




\section{Appendix 6}

Detailed array results from experiments A and B. The tables depict statistical significant differentially expressed miRNAs for each of the comparisons mentioned in the text (Results, Section 3.8.). Values in the second column demonstrate fold change $2^{\mathrm{X}}$, where $\mathrm{X}$ is the number in red or green. Each comparison has the format 'cell line A-cell line B'. Red means that $\mathrm{B}>\mathrm{A}$, green that $\mathrm{B}>\mathrm{A}$. Padj $=$ the adjusted $\mathrm{P}$ value. Expr $=$ Experiment.

\section{ESC Stra8-maGSC Stra8 Expr A}

\begin{tabular}{|c|c|c|}
\hline Gene Name & $\begin{array}{l}\frac{\text { ESC Stra8- }}{\text { maGSC Stra8 }} \\
\underline{\text { ExprA }}\end{array}$ & Padj \\
\hline miRPlus_17952 & -6.0 & 0.00479 \\
\hline mmu-miR-762 & -5.7 & 0.00404 \\
\hline miRPlus_21472 & -5.0 & 0.00304 \\
\hline mmu-miR-712* & -4.9 & 0.0015 \\
\hline hsa-miR-193a-5p & -4.7 & 0.00159 \\
\hline hsa-miR-663 & -4.6 & 0.00404 \\
\hline hsa-miR-30b* & -4.6 & 0.0022 \\
\hline mmu-miR-720 & -4.5 & 0.0078 \\
\hline hsa-miR-623 & -4.5 & 0.00658 \\
\hline hsa-miR-492 & -4.4 & 0.00488 \\
\hline hsa-miR-671-5p & -4.4 & 0.00396 \\
\hline miRPlus_17890 & -4.4 & 0.0015 \\
\hline hsa-miR-498 & -4.3 & 0.0013 \\
\hline mmu-miR-711 & -4.3 & 0.00159 \\
\hline hsa-miR-371-5p & -4.2 & 0.0015 \\
\hline miRPlus_27561 & -4.2 & 0.00788 \\
\hline hsa-miR-602 & -4.1 & 0.00304 \\
\hline miRPlus_28431 & -4.0 & 0.00612 \\
\hline miRPlus_27560 & -3.9 & 0.0013 \\
\hline mmu-miR-689 & -3.7 & 0.00188 \\
\hline rno-miR-542-5p & -3.5 & 0.00396 \\
\hline hsa-miR-557 & -3.2 & 0.00854 \\
\hline miRPlus_17921 & -3.0 & 0.0166 \\
\hline hsa-miR-518c* & -2.9 & 0.0015 \\
\hline miRPlus_28575 & -2.8 & 0.00902 \\
\hline hsa-miR-638 & -2.7 & 0.0207 \\
\hline mmu-miR-184 & -2.6 & 0.0035 \\
\hline
\end{tabular}




\begin{tabular}{|l|l|l|}
\hline hsa-miR-503 & -2.5 & 0.00733 \\
\hline mmu-miR-301a & -2.4 & 0.0133 \\
\hline mmu-miR-21 & -2.4 & 0.00614 \\
\hline miRPlus_27564 & -2.3 & 0.00316 \\
\hline mmu-miR-494 & -2.2 & 0.00159 \\
\hline hsa-miR-302c* & -2.2 & 0.0295 \\
\hline rno-miR-376b-3p & 2.0 & 0.00658 \\
\hline
\end{tabular}

\section{$\underline{\text { maGSC Stra8-maGSC129SV ExprA }}$}

\begin{tabular}{|c|c|c|}
\hline$\underline{\text { Gene Name }}$ & $\begin{array}{l}\text { maGSC Stra8- } \\
\text { maGSC129SV } \\
\text { ExprA }\end{array}$ & Padj \\
\hline $\begin{array}{l}\text { mmu-miR-335- } \\
5 p\end{array}$ & -4.2 & 0.0334 \\
\hline mmu-miR-124 & -3.9 & 0.00833 \\
\hline mmu-miR-182 & -3.8 & 0.00628 \\
\hline mmu-miR-96 & -3.7 & 0.0072 \\
\hline mmu-miR-183 & -2.6 & 0.00896 \\
\hline hsa_SNORD13 & -2.5 & 0.00546 \\
\hline $\begin{array}{l}\text { mmu-miR-292- } \\
3 p\end{array}$ & -2.5 & 0.00546 \\
\hline mmu-miR-295 & -2.3 & 0.00809 \\
\hline mmu-miR-22 & -2.2 & 0.0229 \\
\hline mmu-miR-294 & -2.0 & 0.0167 \\
\hline mmu-miR-711 & 2.1 & 0.00905 \\
\hline mmu-miR-494 & 2.1 & 0.00406 \\
\hline $\begin{array}{l}\text { rno-miR-142- } \\
5 \mathrm{p}\end{array}$ & 2.1 & 0.0285 \\
\hline $\begin{array}{l}\text { mmu-miR-142- } \\
5 \mathrm{p}\end{array}$ & 2.1 & 0.0273 \\
\hline miRPlus_17921 & 2.5 & 0.031 \\
\hline mmu-miR-720 & 2.5 & 0.0399 \\
\hline hsa-miR-638 & 2.5 & 0.0285 \\
\hline mmu-miR-706 & 2.6 & 0.00905 \\
\hline $\begin{array}{l}\text { rno-miR-542- } \\
5 \mathrm{p}\end{array}$ & 2.6 & 0.00908 \\
\hline miRPlus_28431 & 2.9 & 0.0165 \\
\hline
\end{tabular}




\begin{tabular}{|l|l|l}
\hline miRPlus_17890 & 3.1 & 0.00384 \\
\hline hsa-miR-602 & 3.2 & 0.00809 \\
\hline hsa-miR-302d & 3.2 & 0.0214 \\
\hline miRPlus_27561 & 3.3 & 0.0186 \\
\hline miRPlus_17952 & 3.3 & 0.0229 \\
\hline $\begin{array}{l}\text { hsa-miR-671- } \\
\text { 5p }\end{array}$ & 3.4 & 0.00905 \\
\hline hsa-miR-30b* & 3.4 & 0.00679 \\
\hline mmu-miR-689 & 3.5 & 0.00406 \\
\hline miRPlus_27560 & 3.6 & 0.00185 \\
\hline hsa-miR-492 & 3.7 & 0.00905 \\
\hline hsa-miR-623 & 3.7 & 0.0135 \\
\hline hsa-miR-557 & 3.8 & 0.00855 \\
\hline hsa-miR-663 & 3.9 & 0.00855 \\
\hline hsa-miR-193a- & 4.1 & 0.00406 \\
\hline 5p & & 0.0072 \\
\hline miRPlus_21472 & 4.2 & 0.0167 \\
\hline $\begin{array}{l}\text { mmu-miR-142- } \\
\text { 3p }\end{array}$ & 4.3 & 0.00185 \\
\hline hsa-miR-498 & 4.4 & 0.00384 \\
\hline $\begin{array}{l}\text { hsa-miR-371- } \\
\text { 5p }\end{array}$ & 4.4 & 0.00859 \\
\hline mmu-miR-762 & 4.6 & \\
\hline
\end{tabular}

\section{maGSC129SV-ESC RI Expr A}

\begin{tabular}{|l|l|l}
\hline Gene Name & maGSC129SV- & Padj \\
\cline { 2 - 3 } & ESC_RI Expr A & \\
\hline mmu-miR-20a & -3.2 & 0.00482 \\
\hline mmu-miR-17 & -3.2 & 0.00482 \\
\hline hsa-miR-106a & -2.9 & 0.00482 \\
\hline mmu-miR-19b & -2.8 & 0.00482 \\
\hline mmu-miR-20b & -2.6 & 0.0163 \\
\hline mmu-miR-467b & -2.6 & 0.00482 \\
\hline mmu-miR- & -2.4 & 0.00531 \\
\hline 467a*/467d* & & 0.00783 \\
\hline mmu-miR-106a & -2.1 & 0.00936 \\
\hline mmu-miR-805 & -2.0 & 0.0216 \\
\hline mmu-miR-22 & 2.5 & \\
\hline
\end{tabular}




\begin{tabular}{|l|l|l|} 
mmu-miR-689 & 2.6 & 0.00783 \\
\hline hsa_SNORD13 & 2.8 & 0.00482
\end{tabular}

\section{ESC RI-maGSC129 ExprB}

\begin{tabular}{|l|l|l}
\hline Gene Name & ESC RI- & Padj \\
\cline { 2 - 3 } & maGSC129 ExprB & \\
\hline $\begin{array}{l}\text { miRPlus_1789 } \\
9\end{array}$ & -2.2 & 0.0767 \\
\hline mmu-miR-20b & 3.5 & 0.0767 \\
\hline rno-miR-290 & 4.8 & 0.0767 \\
\hline mmu-miR-19b & 5.4 & 0.0767 \\
\hline
\end{tabular}




\section{7. $\quad$ References}

Alisch RS, Jin P, Epstein M, Caspary T, Warren ST (2007): Argonaute2 is essential for mammalian gastrulation and proper mesoderm formation. PLoS Genetics $\underline{3}$, e227

Alonso A, Breuer B, Steuer B, Fischer J (1991): The F9-EC cell line as a model for the analysis of differentiation. Int J Dev Biol 35, 389397

Ambros V (2004): The functions of animal microRNAs. Nature $\underline{431}$, 350-355

Andrews PW (2002): From teratocarcinomas to embryonic stem cells. Phil Trans R Soc Lond B Biol Sci $\underline{357}$, 405-417

Andrews PW, Przyborski SA, Thomson J: Embryonal carcinoma cells as embryonic stem cells; in: Stem Cell Biology; edited by Marshak DR, Gardner RL, Gottlieb D; Cold Spring Harbor Laboratory Press, New York/USA 2001, 231-266

Bartel DP (2004): MicroRNAs: Genomics, biogenesis, mechanism, and function. Cell $\underline{116}, 281-297$

Bartel DP, Chen CH (2004): Micromanagers of gene expression: the potentially widespread influence of metazoan microRNAs. Nat Rev Genet $\underline{5}, 396-400$

Boisseau S, Simonneau M (1989): Mammalian neuronal differentiation: early expression of a neuronal phenotype from mouse neural crest cells in a chemically defined culture medium. Development 106, 665-674 
Brook FA, Gardner RL (1997): The origin and efficient derivation of embryonic stem cells in the mouse. Proc Natl Acad Sci U S A $\underline{94}$, 5709-5712

Buitrago W, Roop DR (2007): Oct-4: The almighty POUripotent regulator? J Invest Derm 127, 260-262

Chen C, Ridzon D, Lee CT, Blake J, Sun Y, Strauss WM (2007): Defining embryonic stem cell identity using differentiation-related microRNAs and their potential targets. Mamm Genome 18, 316-327

Chen K, Rajewsky N (2007): The evolution of gene regulation by transcription factors and microRNAs. Nat Rev Genet $\underline{8}, 93-103$

Colucci-Guyon E, Gimenez Y, Ribotta M, Maurice T, Babinet C, Privat A (1999): Cerebellar defect and impaired motor coordination in mice lacking vimentin. Glia $\underline{25}, 33-43$

Conrad S, Renninger M, Hennenlotter J, Wiesner T, Just L, Bonin M, Aicher W, Buehring HS, Mattheus U, Mack A et al. (2008): Generation of pluripotent stem cells from adult human testis. Nature doi:10.1038/nature07404

de Rooij DG, Mizrak SC (2008): Deriving multipotent stem cells from mouse spermatogonial stem cells: a new tool for developmental and clinical research. Development 135, 2207-2213

Duncan SA, Nagy A, Chan W (1997): Murine gastrulation requires HNF-4 regulated gene expression in the visceral endoderm: tetraploid rescue of Hnf-4(-/-) embryos. Development 124, 279-287

Esquela-Kerscher A, Slack FJ (2006): Oncomirs-microRNAs with a role in cancer. Nat Rev Cancer $\underline{6}, 259-269$ 
Evans MJ, Kaufman MH (1981): Establishment in culture of pluripotential cells from mouse embryos. Nature 292, 154-156

Franke WW, Grund C, Kuhn C, Jackson BW, Illmensee K (1982): Formation of cytoskeletal elements during mouse embryogenesis. III. Primary mesenchymal cells and the first appearance of vimentin filaments. Differentiation $\underline{23}$, 43-59

Guan K, Nayernia K, Maier LS, Wagner S, Dressel R, Lee JH, Nolte J, Wolf F, Li M, Engel W, Hasenfuss G (2006): Pluripotency of spermatogonial stem cells from adult mouse testis. Nature $\underline{440}, 1199-$ 1203

Hayashi K, Chuva de Sousa Lopes SM, Kaneda M, Tang F, Hajkova P, Lao K, O Carroll D, Das PP, Tarakhovsky A, Miska EA, Surani MA (2008): MicroRNA biogenesis is required for mouse primordial germ cell development and spermatogenesis. PLoS ONE $\underline{3}$, e1738

He L, Hannon GJ (2004): MicroRNAs: small RNAs with a big role in gene regulation. Nature Rev Genet $\underline{5}, 522-531$

He Z, Jiang J, Hofmann MC, Dym M (2007): Gfral silencing in mouse spermatogonial stem cells results in their differentiation via the inactivation of RET tyrosine kinase. Biol Reprod 77, 723-733

Hogan B: Primordial germ cells as stem cells; in: Stem Cell Biology; edited by Marshak DR, Gardner RL, Gottlieb D; Cold Spring Harbor Laboratory Press, New York/USA 2001, 189-204

Houbaviy H, Murray M, Sharp P (2003): Embryonic stem cellspecific microRNAs. Dev Cell $\underline{5}, 351-358$ 
Houbaviy H, Dennis L, Jaenisch R, Sharp PA (2005): Characterization of a highly variable eutherian microRNA gene. RNA $\underline{11}, 1245-1257$

http://microrna.sanger.ac.uk/ (2008)

Itman C, Loveland KL (2008): SMAD expression in the testis: an insight into BMP regulation of spermatogenesis. Dev Dyn 237, 97111

Izadyar F, Pau F, Marh J, Slepko N, Wang T, Gonzalez R, Ramos T, Howerton K, Sayre C, Silva F (2008): Generation of multipotent cell lines from a distinct population of male germ line stem cells. Reproduction 135, 771-784

Johnson SM, Grosshans H, Shingara J (2005): RAS is regulated by the let-7 microRNA family. Cell $\underline{120}, 635-647$

Kanatsu-Shinohara M, Inoue K, Lee J, Yoshimoto M, Ogonuki N, Miki H, Baba S, Kato T, Kazuki Y, Toyokuni S, Toyoshima M, Niwa O, Oshimura M, Heike T, Nakahata T, Ishino F, Ogura A, Shinohara $\mathrm{T}$ (2004): Generation of pluripotent stem cells from neonatal mouse testis. Cell $\underline{119}, 1001-1012$

Kanatsu-Shinohara M, Lee J, Inoue K, Ogonuki N, Miki H, Toyokuni S, ikawa M, Tomoyuki N, Ogura A, Shinohara T (2008): Pluripotency of a single spermatogonial stem cell in mice. Biol Reprod $\underline{78}, 681-687$

Kanellopoulou C, Muljo SA, Kung AL, Ganesan S, Drapkin R, Jenuwein T, Livingston DM, Rajewsky K (2005): Dicer-deficient mouse embryonic stem cells are defective in differentiation and centromeric silencing. Genes Dev 19, 489-510 
Keller G (2005): Embryonic stem cell differentiation: emergence of a new era in biology and medicine. Genes Dev $\underline{19}, 1129-1155$

Kim VN (2005): MicroRNA biogenesis: coordinated cropping and dicing. Nat Rev Mol Cell Biol $\underline{6}, 376-385$

Kirschstein R, Skirboll LR: Stem cells: Scientific progress and future research directions; in: NIH stem cells, National Institute of Health, Bethesda, Maryland/USA 2001, 7-8

Kurosawa H (2007): Methods for inducing embryoid body formation: in vitro differentiation system of embryonic stem cells. J Biosci Bioeng 103, 389-398

Lacham-Kaplan O (2004): In vivo and in vitro differentiation of male germ cells in mouse. Reproduction $\underline{128}, 147-152$

Lagos-Quintana M, Rauhut R, Lendeckel W, Tuschl T (2001): Identification of novel genes coding for small expressed RNAs. Science 294, 853-858

Lagos-Quintana M, Rauhut R, Yalcin A, Meyer J, Lendeckel W, Tuschl (2002): Identification of tissue specific miRNAs from mouse. Curr Biol 12, 735-739

Laurent LC, Chen J, Ulitsky I, Mueller FJ, Lu C, Shamir R, Fan JB, Loring JF (2008): Comprehensive microRNA profiling reveals a unique human embryonic stem cell signature dominated by a single seed sequence. Stem Cells $\underline{26}, 1506-1516$

Lendahl U (1997): Transgenic analysis of central nervous system development and regeneration. Acta Anaesthesiol Scand Suppl 110, 116-118 
Lensch MW, Daheron L, Schlaeger TM (2006): Pluripotent stem cells and their niches. Stem Cell Rev 2, 185-201

Liberatore CM, Searcy-Schrick RD, Vincent EB, Yutzey KE (2002): Nkx-2.5 gene induction in mice is mediated by a Smad consensus regulatory region. Dev Biol 244, 243-256

Lin RC, Matesie DF, Marvin M, McKay RD, Bruestle O (1995): Reexpression of the intermediate filament nestin in reactive astrocytes. Neurobiol Dis $\underline{2}, 79-85$

Liu N, Lu M, Tian X, Han Z (2007): Molecular mechanisms involved in self-renewal and pluripotency of embryonic stem cells. J Cell Physiol 211, 279-286

Marshak DR, Gardner RL, Gottlieb D: Introduction: Stem Cell Biology, 1; in: Stem Cell Biology; edited by Marshak DR, Gardner RL, Gottlieb D; Cold Spring Harbor Laboratory Press, New York/USA 2001, 1-16

Masui S, Nakatake Y, Toyooka Y, Shimosato D, Yagi R, Takahashi K, Okochi H, Okuda A, Matoba R, Sharov AA, Ko MS, Niwa H (2007): Pluripotency governed by Sox 2 via regulation of Oct3/4 expression in mouse embryonic stem cells. Nat Cell Biol $\underline{9}, 625-635$

Matsuda T, Nakamura T, Nakao K, Arai T, Katsuki M, Heike T, Yokota T (1999): STAT3 activation is sufficient to maintain an undifferentiated state of mouse embryonic stem cells. EMBO J $\underline{18}$, 4261-4269

Meissner A, Werning M, Jaenisch R (2007): Direct reprogramming of genetically unmodified fibroblasts into pluripotent stem cells. Nat Biotechnol 25, 1177-1181 
Mendell JT (2008): miRiad roles for the miR-17-92 cluster in development and disease. Cell 133, 217-222

Meyer S, Goettingen: Unpublished data 2008

Nayernia K, Li M, Jaroszynski L, Khusainov R, Wulf G, Schwandt I, Korabiowska M, Michelmann HW, Meinhardt A, Engel W (2004): Stem cell based therapeutical approach of male infertility by teratocarcinoma derived germ cells. Hum Mol Genet 13, 1451-1460

Nichols J, Zevnik B, Anastassiadis K, Niwa H, Klewe- Nebenius D, Chambers I, Scholer H, Smith A (1998): Formation of pluripotent stem cells in the mammalian embryo depends on the POU transcription factor Oct4. Cell 95, 379-391

Nishikawa S, Hirashima M, Matsuyoshi N, Kodama H (1998): Progressive lineage analysis by cell sorting and culture identifies FLK+VE-cadherin cells at a diverging point of endothelial and hemopoietic lineages. Development 125, 1747-1757

Niwa H, Burdon T, Chambers I, Smith A (1998): Self-renewal of pluripotent embryonic stem cells is mediated via activation of STAT3. Genes Dev 12, 2048-2060

Pan G, Thomson JA (2007): Nanog and transcriptional networks in embryonic stem cell pluripotency. Cell Res 17, 42-49

Pelton TA, Bettess MD, Lake J, Rathjen J, Rathjen PD (1998): Developmental complexity of early mammalian pluripotent cell populations in vivo and in vitro. Reprod Fertil Dev 10, 535-549 
Pesce M, Anastassiadis K, Scholer HR (1999): Oct-4: lessons of totipotency from embryonic stem cells. Cells Tissues Organs $\underline{165}$, 144-152

Ralston A, Rossant J (2005): Genetic regulation of stem cell origins in the mouse embryo. Clin Genet $\underline{68}, 106-112$

Rao M (2004): Conserved and divergent paths that regulate selfrenewal in mouse and human embryonic stem cells. Dev Biol $\underline{275}$, 269-286

Reik W, Dean W, Walter J (2001): Epigenetic reprogramming in mammalian development. Science 293, 1089-1093

Rodda DJ, Chew JL, Lim LH, Loh YH, Wang B, Ng HH, Robson P (2005): Transcriptional regulation of Nanog by Oct4 and Sox2. J Biol Chem 280, 24731-24737

Rohwedel J, Sehlmeyer U, Shan J, Meister A, Wobus AM (1996): Primordial germ cell-derived mouse embryonic germ (EG) cells in vitro resemble undifferentiated stem cells with respect to differentiation capacity and cell cycle distribution. Cell Biol Int $\underline{20}$, 279-587

Rohwedel J, Guan K, Wobus A (1999): Induction of cellular differentiation by retinoic acid in vitro. Cells Tissues Organs $\underline{165}$, 190-202

Schoeler HR, Hatzopoulos AK, Balling R, Suzuki N, Gruss P (1989): A family of octamer-specific proteins present during mouse embryogenesis: evidence for germline-specific expression of an Oct factor. EMBO J $\underline{8}, 2543-2550$ 
Seandel M, James D, Shmelkov SV, Falciatori I, Kim J, Chavala S, Scherr DS, Zhang F, Torres R, Gale NW, Yancopoulos GD, Murphy A, Valenzuela DM, Hobbs RM, Pandolfi PP, Rafii S (2007): Generation of functional multipotent adult stem cells from GPR125+ germline progenitors. Nature $\underline{449}, 346-350$

Showell C, Binder O, Conlon FL (2003): T- box Genes in early embryogenesis. Dev Dyn 229, 201-218

Sinkkonen L, Hugenschmidt T, Berninger P, Gaidatzis D, Mohn F, Artus-Revel CG, Zavolan M, Svoboda P, Filipowicz W (2008): MicroRNAs control de novo DNA methylation through regulation of transcriptional repressors in mouse embryonic stem cells. Nature Struct Mol Biol 15, 259-267

Strauss W, Chen C, Lee CT, Ridzon D (2006): Nonrestrictive developemental regulation of microRNA gene expression. Mamm Genome 17, 833-840

Suh MR, Lee Y, Kim JY, Kim SK, Moon SH, Lee JY, Cha KY, Chung HM, Yoon HS, Moon SY, Kim VN, Kim KS (2004): Human embryonic stem cells express a unique set of microRNAs. Dev Biol $\underline{270}, 488-498$

Tam PPL, Loebel DAF (2007): Gene function in mouse embryogenesis: get set for gastrulation. Nat Rev Genet $\underline{8}, 368-381$

Tang F, Kaneda M, O'Carroll D, Hajkova P, Barton SC, Sun YA, Lee C, Tarakhovsky A, Lao K, Surani MA (2007): Maternal microRNAs are essential for mouse zygotic developement. Genes Dev 21, 644-648 
Taraviras S, Monaghan AP, Schütz G, Kelsey G (1994): Characterization of the mouse HNF-4 gene and its expression during mouse embryogenesis. Mech Dev $\underline{48}, 67-79$

Tay Y, Zhang J, Thomson AM, Lim B, Rigoutsos I (2008): MicroRNAs to Nanog, Oct4 and Sox2 coding regions modulate embryonic stem cell differentiation. Nature doi:10.1038/nature07299

Tegelenbosch RA, de Rooij DG (1993): A quantitative study of spermatogonial multiplication and stem cell renewal in the $\mathrm{C} 3 \mathrm{H} / 101$ F1 hybrid mouse. Mutat Res 290, 193-200

Tighe A, Gudas LJ (2004): Retinoic acid inhibits leukaemia inhibitory factor signaling pathways in mouse embryonic stem cells. J Cell Physiol 198, 223-229

Wang ZX, Teh Ch, Kueh JL, Lufkin T, Robson P, Stanton LW (2007): Oct4 and Sox2 directly regulate expression of another pluripotency transcription factor, Zfp206, in embryonic stem cells. J Biol Chem 282, 12822-12830

Wiese C, Rolletschek A, Kania G, Blyszczuk P, Tarasov KV, Tarasova Y, Wersto RP, Boheler KR, Wobus AM (2004): Nestin expression-a property of multi-lineage progenitor cells? Cell Mol Life Sci $\underline{61}, 2510-2522$

Wurst W, Joyner AL: Production of targeted embryonic stem cell clones; in: Gene targeting: a practical approach; edited by Joyner AL; IRL Press, Oxford/UK 1993, 33-61

Zechner U, Mainz: Unpublished data 2008 


\section{Related Publications}

Part of this study has been published as follows (next page): 


\title{
Multipotent adult germline stem cells and embryonic stem cells have similar microRNA profiles
}

\author{
Athanasios Zovoilis' ${ }^{1,5}$, Jessica Nolte ${ }^{1}$, Nadja Drusenheimer ${ }^{1}$, Ulrich Zechner ${ }^{2}$, Hiroki Hada ${ }^{1}$, \\ Kaomei Guan ${ }^{3}$, Gerd Hasenfuss ${ }^{3}$, Karim Nayernia ${ }^{4}$ and Wolfgang Engel ${ }^{1}$
}

\author{
${ }^{1}$ Institute of Human Genetics, University of Goettingen, Heinrich-Dueker-Weg 12, Goettingen D-37073, Germany; ${ }^{2}$ Institute of Human \\ Genetics, University of Mainz, Mainz, 55131, Germany; ${ }^{3}$ Department of Cardiology and Pneumology, University of Goettingen, \\ Goettingen 37075, Germany; ${ }^{4}$ Institute of Human Genetics, International Centre for Life, University of Newcastle, Newcastle upon Tyne \\ NE1 3BZ, UK \\ ${ }^{5}$ Correspondence address. Tel: +49-551-397589; Fax: +49-551-399303; E-mail: azovoil@gwdg.de
}

\begin{abstract}
Spermatogonial stem cells (SSCs) isolated from the adult mouse testis and cultured have been shown to respond to culture conditions and become pluripotent, so called multipotent adult germline stem cells (maGSCs). microRNAs (miRNAs) belonging to the 290 and 302 miRNA clusters have been previously classified as embryonic stem cell (ESC) specific. Here, we show that these miRNAs generally characterize pluripotent cells. They are expressed not only in ESCs but also in maGSCs as well as in the F9 embryonic carcinoma cell (ECC) line. In addition, we tested the time-dependent influence of different factors that promote loss of pluripotency on levels of these miRNAs in all three pluripotent cell types. Despite the differences regarding time and extent of differentiation observed between ESCs and maGSCs, expression profiles of both miRNA families showed similarities between these two cell types, suggesting similar underlying mechanisms in maintenance of pluripotency and differentiation. Our results indicate that the 290-miRNA family is connected with Oct-4 and maintenance of the pluripotent state. In contrast, members of the 302-miRNA family are induced during first stages of in vitro differentiation in all cell types tested. Therefore, detection of miRNAs of miR-302 family in pluripotent cells can be attributed to the proportion of spontaneously differentiating cells in cultures of pluripotent cells. These results are consistent with ESC-like nature of maGSCs and their potential as an alternative source of pluripotent cells from non-embryonic tissues.
\end{abstract}

Keywords: multipotent adult germline stem cells; embryonic stem cells; microRNAs; Oct4; pluripotency markers

\section{Introduction}

Embryonic stem cells (ESCs) are known to be pluripotent cells having the capacity to self-renew as well as the ability to generate all types of differentiated cells. However, ESCs face immune reaction after transplantation and there are ethical issues regarding the usage of embryos. Several studies have revealed that the germline lineage retains the potential to generate pluripotent cells. In 2004, ESC-like cells were found in germ stem cell cultures established from neonatal mouse testis, designated as multipotent germline stem cells (KanatsuShinohara et al., 2004). In 2006, we have isolated and cultured for the first time spermatogonial stem cells (SSCs) from the adult mouse testis which respond to culture conditions and acquire ESC properties (Guan et al., 2006). We proved that the pluripotency and plasticity of these cells, which were named multipotent adult germline stem cells (maGSCs), were similar to ESCs. They are able to spontaneously differentiate into derivatives of the three embryonic germ layers in vitro, to generate teratomas in immunodeficient mice and to contribute to the development of various organs when injected into an early blastocyst. Isolation of these cells is not restricted to the transgenic Stra8-EGFP/ROSA26 mouse. We have successfully obtained ESC-like cell lines derived from testes of three different strains of mice (FVB, C57BL/6 and 129/Sv) by morphological criteria only. Our results were confirmed by other groups (Seandel et al., 2007;
Izadyar et al., 2008). Interestingly, another group showed recently that SSCs are not pluripotent but that a single SSC can dedifferentiate from a highly lineage-specified state to a pluripotent state (KanatsuShinohara et al., 2008). Since pluripotent cells have not been reported for human testes until now, the mouse is a necessary model system for the study of these cells.

In this study, we were interested to substantiate the ESC-like nature of maGSCs with respect to microRNA (miRNA) expression. miRNAs represent a recently identified class of cellular RNAs that regulate protein expression at the translational level. The mature miRNAs are 17-24 bp single-stranded RNA molecules which are expressed in eucaryotic cells and affect the translation or stability of target mRNAs (Bartel, 2004; Bartel and Chen, 2004). Each miRNA seems to be able to regulate multiple genes. It was shown recently that the expression of certain genes is more dependent on the level of regulatory miRNAs than on the level of mRNAs that encode the proteins (Johnson et al., 2005).

Recently, a set of miRNAs was described to be ESC-specific in mouse, with their expression being repressed during ESC differentiation and undetectable in adult mouse organs. This set of miRNAs consists of miR-290, miR-291a-3p, miR-292-3p, miR-293, miR-294 and miR-295 (miR-290 family), and miR-302a, miR-302b, miR-302c and miR-302d (miR-302 family). In a previous work, 
miRNAs of the miR-290 family were repressed in embryoid bodies (EBs) prepared by culturing ESC for 14 days in either the presence or absence of retinoic acid (RA), and it was suggested that their expression is specific for pluripotent ES cells and is either silenced or down-regulated upon differentiation (Houbaviy et al., 2003, 2005). Another group confirmed the expression of these miRNAs as well as of those of miR-302 family only in mESCs and mEBs, and not in somatic tissues. In addition, they reported a negative correlation in EBs between miRNAs of miR-302 family and differentiation time (Strauss et al., 2006; Chen et al., 2007). These miRNAs are expressed in clusters (members of each miR-family are transcribed as parts of the same pri-miRNA) and they have close homologues in human ESCs with the same expression profile during differentiation (Suh et al., 2004). However, their role in pluripotency is still not well defined. In this study, we show that these miRNAs generally characterize pluripotent cells, since maGSCs share with ESCs the unique characteristic of expressing these miRNAs. Furthermore, we show that members of miR-302 family are induced during first stages of in vitro differentiation.

\section{Materials and Methods}

\section{Culture of mouse maGSC and ESC lines}

The culture of maGSC lines from mouse lines 129/Sv (maGSC 129SV), C57BL/6 (maGSC C57BL), FVB (maGSC FVB) and from the transgenic line Stra8-EGFP/ROSA26 (maGSC Stra8) was described previously (Guan et al., 2006). The ESC R1 line was derived from the $129 / \mathrm{Sv}$ mouse line (Wurst and Joyner, 1993). The ESC line ESC Stra8 was generated from the transgenic Stra8-EGFP/ROSA26 mouse as described previously (Cheng et al., 2004). To maintain maGSCs and ESCs in an undifferentiated state, the cells were cultured under standard ESC culture conditions: DMEM (PAN, Aidenbach, Germany) supplemented with $20 \%$ fetal calf serum (PAN), $2 \mathrm{mM}$ L-glutamine (Pan), $50 \mu \mathrm{M} \beta$-mercaptoethanol (Gibco/Invitrogen, Eggenstein, Germany), $1 \times$ non-essential amino acids (Gibco/Invitrogen), sodium pyruvate (Gibco/Invitrogen), penicillin/streptomycin (PAN). maGSCs and ESCs were cultured on a feeder layer of mitomycin C-inactivated mouse embryonic fibroblasts (MEFs) in the presence of $1000 \mathrm{U} / \mathrm{ml}$ recombinant mouse leukaemia inhibitory factor (LIF) (Chemicon, Temecula, USA). For the differentiation studies, the following culture conditions were used: (A) ESC medium with fibroblasts (FL) and LIF (FL+LIF); (B) ESC medium with FL, LIF and RA $\left(10^{-6} \mathrm{M}\right)$ (Sigma-Aldrich, Steinheim, Germany) (FL+ LIF+RA); (C) cells were cultured in $0.1 \%$ gelatine-coated culture flasks with ESC medium, without LIF (Gel); (D) cells cultured in $0.1 \%$ gelatinecoated culture flasks with ESC medium, without LIF but with RA $(\mathrm{Gel}+\mathrm{RA})$. In order to eliminate the impact of FL on the accuracy of the results from cells cultured under conditions $\mathrm{A}$ and $\mathrm{B}$, cells were cultured for 5 days (two passages) on $0.1 \%$ gelatine instead of FL prior to miRNA and protein extraction. F9 cells were obtained from ATCC (Manassas, USA) and cultured as described previously (Nayernia et al., 2004).

\section{miRNA and mRNA analysis}

Total RNA including miRNAs was isolated from cultured cells and from testes of wild-type 129/Sv mouse using the miRNeasy mini Kit (Qiagen, Hilden, Germany). Conversion of miRNA and mRNA into cDNA and real-time PCR detection of miRNAs was carried out according to the manufacturer's protocols using the miScript Reverse Transcription Kit and miScript SYBR Green PCR Kit (Qiagen) on an ABI Prism 7900HT Sequence Detection System. Optimized miRNA-specific primers for each miRNA as well as for the endogenous control RNU6B are also commercially available (miScript Primer Assays, Qiagen). All experiments were performed in duplicate and PCR specificity was checked by melting curves, gel electrophoresis and sequencing of the PCR products after gel extraction and cloning into a pGEM-T Easy vector (Promega, Madison, USA). On the basis of preliminary results, we decided not to include miR-302c in our study, since the high amount of unspecific products observed for this miRNA could not guarantee reliability of the results. The ESC R1 line was used to prepare the standard curve for both the target miRNA and RNU6B, to which all quantities were further normalized, and as calibrator. The ESC R1 (Fig. 1b) was of a higher passage number of the ESC R1 used in all other experiments. Moreover, RNA from MEFs was used to exclude the possibility of contamination due to FL. For real-time quantitative RT-PCR of Nestin, Vimentin, Hnf4, Nkx2.5 and Sdha, to which all quantities were further normalized, the QuantiTect SYBR-Green PCR MasterMix (Qiagen) was used with gene-specific primers provided in Supplementary Table S1.

\section{Protein isolation, western blotting and immunofluorescence}

For isolation of proteins from cultured cells, cell pellets were resuspended in lysis buffer (10 mM Tris/HCl, $\mathrm{pH} 8,1 \mathrm{mM}$ EDTA, 2.5\% SDS) containing $1 \mathrm{mM}$ phenylmethanesulphonylfluoride and proteinase inhibitors and were sonificated. For protein isolation from mouse testis, $30 \mathrm{mg}$ of tissue was homogenized in the lysis buffer. Protein extracts $(20 \mu \mathrm{g})$ were denaturated at $70^{\circ} \mathrm{C}$ in NuPage SDS sample buffer (Invitrogen, Karlsruhe, Germany)

\section{a}

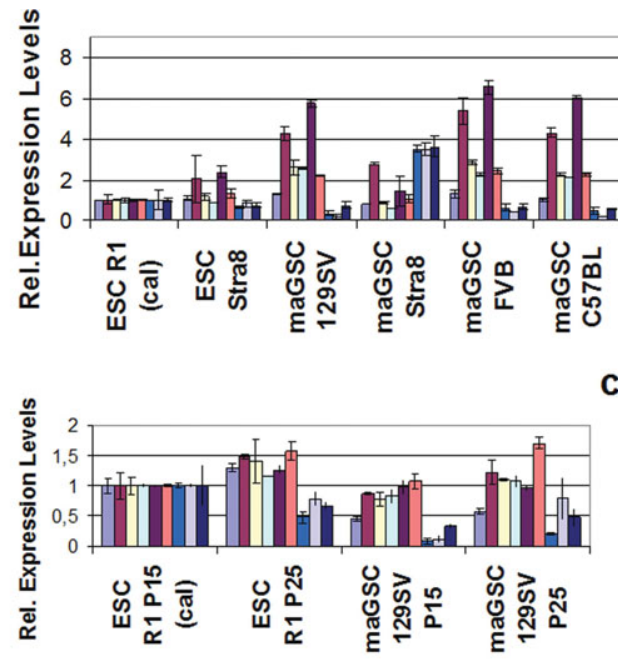

C

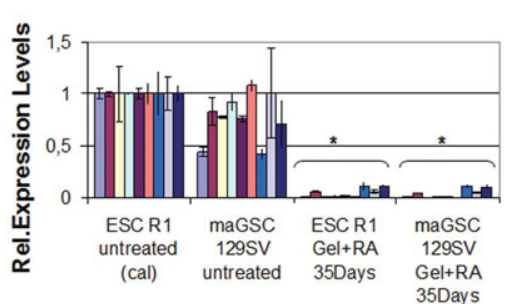

Figure 1: Expression levels of ESC-specific miRNAs detected by real-time PCR.

(a) miRNA expression in different maGSC lines, F9 cells, MEFs, NIH/3T3 cells and testis. ESC R1 (of a different passage number of that used in all other experiments) was used as calibrator (cal). (b) miRNA expression levels in ESC R1 and maGSC 129SV of passages 15 and 25. (c) miRNA expression levels in untreated ESC R1 and maGSC 129SV cells compared with the respective cells cultured for 35 days in gelatine-coated flasks without LIF but with RA (Gel+RA). Asterisks indicate statistical significance. 
containing $0.1 \mathrm{mM}$ dithiothreitol (DTT), separated on NuPage 10\% Bis-Tris Gel (Invitrogen) and transferred on a Hybond-C extra membrane (GE Healthcare Europe, Freiburg, Germany). Blots were blocked for unspecific binding and were incubated overnight at $4^{\circ} \mathrm{C}$ with primary and for $1 \mathrm{~h}$ at $4{ }^{\circ} \mathrm{C}$ with secondary HRP-conjugated antibody. Protein bands were visualized using enhanced chemiluminescence as described by the manufacturer (Santa Cruz Biotechnology, USA). When the expected band size allowed it, membranes were reused for one more time and were incubated with another primary antibody after blocking. The following antibodies were used: $\alpha$-Tubulin dilution 1:5000 (Sigma-Aldrich, T5168), anti-rabbit and anti-mouse IgG-peroxidase antibodies (Sigma-Aldrich), Oct-4 dil 1:500 (Abcam, Cambridge, UK, ab19857), Sox-2 dil 1:1000 (Abcam, ab15830), Zfp-206 (gift from Dr L. Stanton, Singapore) and Sall-4 dil 1:500 (Abcam, ab29112). For immunofluorescence staining of SSEA-1, the ES Cell Characterization Kit (Chemicon) was used as described by the manufacturer. An anti-rabbit IgG Cy3-conjugated antibody (Sigma-Aldrich) was used as secondary antibody and slides were stained with DAPI (Vectashield, Vector Laboratories, Burlingame, USA). Slides were viewed in a BX60 fluorescence microscope (Olympus, Hamburg, Germany). Levels of Oct-4 and Sox-2 from western blots of two independent experiments were quantified densitometrically with QuantityOne software (Bio-Rad, Muenchen, Germany) and normalized to $\alpha$-Tubulin.

\section{Statistical analysis}

Data are expressed as the mean $\pm \mathrm{SD}$. A one-way analysis of variance (ANOVA) followed by Fisher LSD's multiple comparison tests was used for statistical analysis with $P<0.05$ considered statistically significant.

\section{Results}

\section{maGSCs express standard pluripotency markers as well as Sall-4 and Zfp-206}

In order to evaluate the pluripotency of the cells used in the experiments, the expression of pluripotency markers Oct-4, Sox-2, Zfp-206 and Sall-4 was determined at the protein level (Schöler et al., 1989; Rodda et al., 2005; Buitrago and Roop, 2007; Masui et al., 2007; Pan and Thomson, 2007). Zfp-206 and Sall-4 have been shown recently to be expressed in ESCs and become downregulated during ESC differentiation (Zhang et al., 2006; Wang et al., 2007). As it can be seen in Supplementary Fig. S1a, b and $\mathrm{S} 2 \mathrm{a}$, the pluripotency markers are highly expressed in all maGSC lines derived from different mouse strains (maGSC 129SV, maGSC Stra8, maGSC FVB and maGSC C57BL). The expression of Oct-4, Sox-2 in ESC R1 and ESC Stra8 and of Sall-4 and Zfp-206 in ESC $\mathrm{R} 1$ was used as control. The pluripotency marker proteins could not be detected by western analysis in testis nor in inactivated MEFs.

As can be seen in Supplementary Fig. S2a, the expression of Oct-4 and Sox-2 in ESC and maGSC lines remains unchanged during passages $15-25$. When cultured under differentiation conditions for 35 days (cells in $0.1 \%$ gelatine-coated flasks with $10^{-6} \mathrm{M} \mathrm{RA}$ ), Oct-4, Sox-2 and SSEA-1 are down-regulated in ESC lines as well as in maGSC lines (Supplementary Fig. S2a and b).

\section{ESC-specific miRNAs are expressed in maGSCs}

A specific set of miRNAs is known to be present in pluripotent ESCs. These miRNAs can be demonstrated in ESC and maGSC lines of different mouse strains, whereas no expression was detected in MEFs, NIH 3T3 cells and testis (Fig. 1a). Interestingly, miRNA expression pattern of maGSC Stra8 differed from maGSCs derived from other mouse strains by demonstrating lower and higher levels of miR-290 and miR-302 family, respectively. In addition, differences were observed between maGSC 129SV and ESCs from the same mouse strain (ESC R1). We examined whether ESC and maGSC lines retain the expression of the specific miRNAs after culture for many passages. Cells of passage 15 from the mouse strain $129 / \mathrm{Sv}$ were cultivated for 35 days (10 passages; P25) under standard ESC culture conditions. miRNA expression levels were found to remain relatively stable despite slight differences between both cell types (Fig. 1b). Under differentiation conditions for 35 days (cells on $0.1 \%$ gelatine in the presence of $10^{-6} \mathrm{M}$ RA), however, ESCs as well as maGSCs lost their specific miRNA signature (Fig. 1c).

\section{Members of miR-290 family are connected with maintenance of pluripotency}

We examined the effects of different factors that are commonly used for in vitro differentiation of pluripotent cells on the expression of members of ESC-specific miRNA families 290 and 302 in maGSCs in comparison with ESCs. Figure 2a summarizes the strategy we followed. ESCs and maGSCs of passage P16 from the mouse strain 129/Sv (ESC R1 and maGSC 129SV, respectively) were cultivated for 5, 10 and 21 days under different culture conditions: feeder layer (FL), LIF and RA (FL+LIF+RA); $0.1 \%$ gelatine-coated flasks (Gel); $0.1 \%$ gelatine-coated flasks and RA (Gel+RA). We also studied cells that were cultivated for 5 days in $0.1 \%$ gelatine-coated flasks and then induced by RA for 5 days (Gel+RA from Day 5). Cells were collected at Day 5, 10 and 21 and expression of miRNAs was determined.

To assess the degree of differentiation, we determined the levels of Oct- 4 and Sox-2 proteins by western analysis, and the expression of differentiation markers like Nestin, Vimentin, Hnf4 and Nkx2.5 was analysed by qRT-PCR. Figure $2 b$ shows that, after 5 days under FL+ LIF+RA condition, the expression of Oct- 4 and Sox- 2 is strongly down-regulated in ESCs and maGSCs. After culture of the cells for 5 days under Gel+RA condition, Oct- 4 expression is hardly detectable in maGSCs and absent in ESCs. Furthermore, no Sox-2 expression is detected. However, culture under Gel condition for 5 days was found to result in down-regulation of both pluripotency marker proteins only in ESCs, but not in maGSCs. In maGSCs, Oct-4 protein levels are similar to those of untreated cells. Only after cultivation of maGSCs under Gel condition for a longer period (21 days), Oct-4 expression is down-regulated (Fig. 2c).

Expression pattern of the differentiation markers tested differed between ESCs and maGSCs. In ESCs (Fig. 3a), Vimentin and Nestin are significantly increased under Gel+RA condition at Day 5 (Vimentin also under FL+LIF+RA condition), and in all three differentiation conditions at Day 10. At Day 21, they are down-regulated under all conditions, and only Nkx2.5 is increased under Gel and Gel+RA conditions at that day. Expression of Hnf4 is significantly up-regulated only at Day 5 under Gel+RA condition. In contrast in maGSCs (Fig. 3b), no significant change in the expression of these markers takes place at Day 5 and 10, with the exceptions of Nestin, Vimentin and Hnf4 under FL+LIF+RA condition at Day 10 and a slight increase of Nkx2.5 under Gel condition at Day 10. Only at Day 21, an increase of Vimentin and Nestin expression under Gel+RA condition and of Nestin under Gel condition is observed. At Day 21, expression of Nkx2.5 and Hnf4 were restricted to under Gel and Gel+RA conditions, respectively.

ESCs and maGSCs differed also concerning expression levels between Gel+RA and Gel+RA from Day 5 condition. Compared with Gel+RA condition, levels of Nestin and Vimentin in ESCs were lower when RA was added from Day 5, whereas in maGSCs Nestin, Vimentin and Nkx2.5 were increased under this condition and only Hnf4 levels were lower (Supplementary Fig. S3c).

We then studied the effects of the different culture conditions on the expression of members of miRNA families 290 and 302 in ESCs and maGSCs. Figure 4 shows the expression profile of miRNAs of the 290-family in ESCs and maGSCs during culture (5-21 days) under 


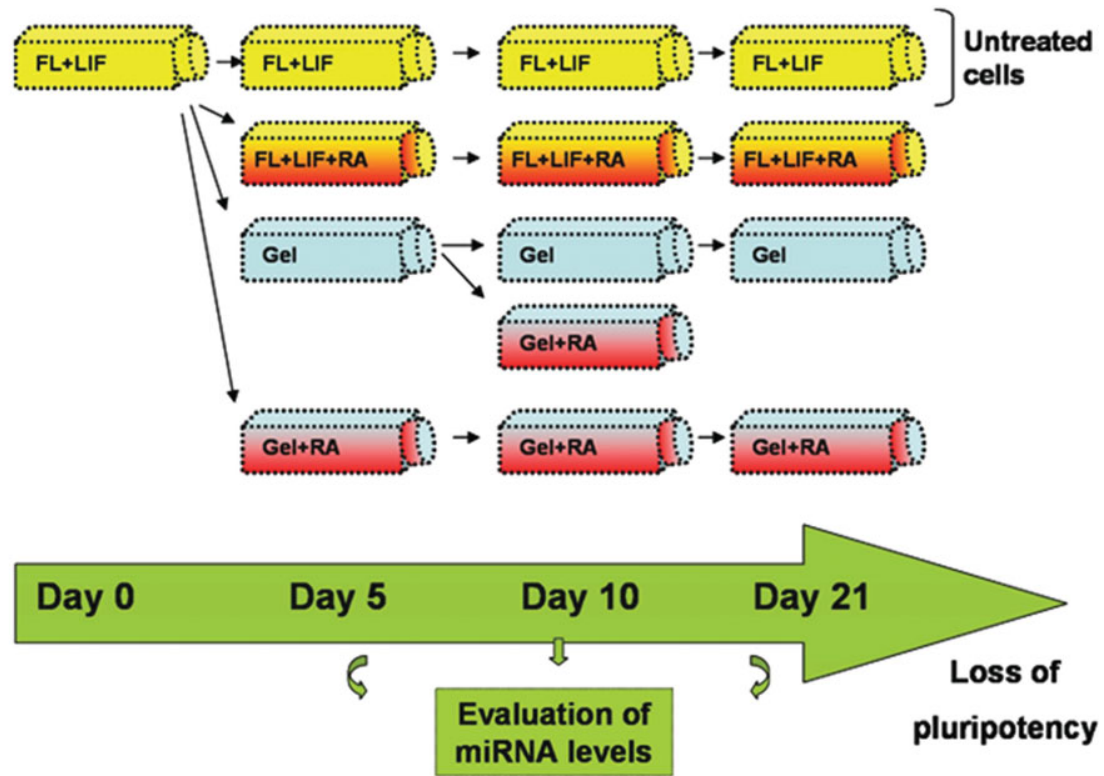

b

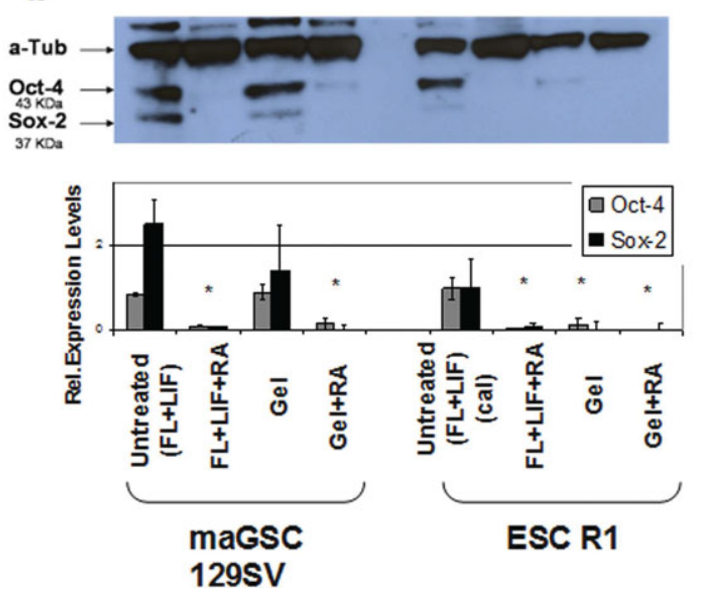

C

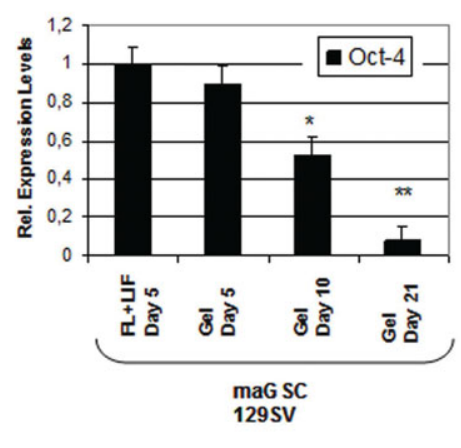

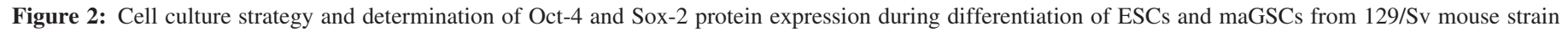
(ESC R1 and maGSC 129SV).

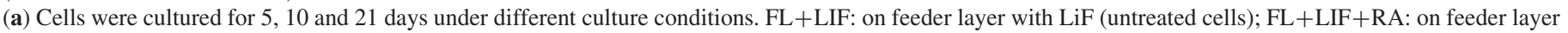

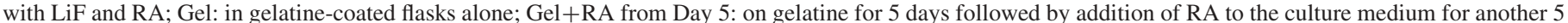

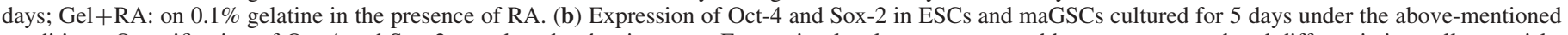

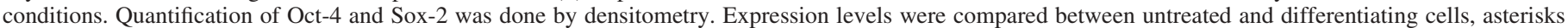

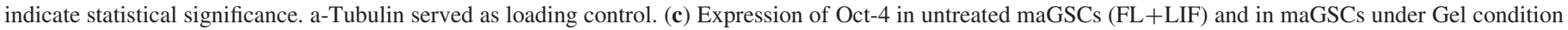

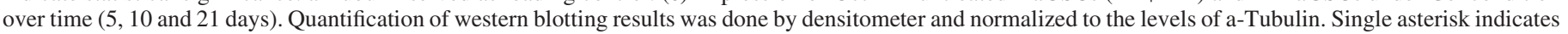
statistical significance between Day 5 and Day 10, double asterisks between Day 10 and Day 21.

all differentiation conditions used as well as in untreated cells. In both ESCs and maGSCs, all members of the 290-family are constantly expressed or even increased in untreated cells, although in the case of maGSCs at lower levels (50\% of ESC expression in some cases) comparing with ESCs. In ESCs, these miRNAs are down-regulated at Day 5 of culture under all differentiation conditions with the strongest effect observed in Gel+RA condition (Fig. 4a). At Day 10 and 21, miRNA levels can hardly be detected under all differentiation conditions (Fig. 4b and c). In maGSCs at Day 5, levels of miRNAs do not decrease in Gel and Gel+RA conditions (with the exception of miR-290 in Gel+RA). Their expression is the same or even higher than in untreated cells (Fig. 4a). At Day 10, miRNA levels have further increased in Gel condition (Fig. 4b). In Gel+RA condition at Day 10, miRNA levels do not increase further but they are still high, whereas a strong down-regulation at Day 10 is observed only under FL+LIF+RA condition (Fig. 4b). At Day 21, miRNA levels of cells in Gel condition are lower than those of untreated cells (with the exception of miR-290), but remain still high in comparison with the other two conditions (Gel+RA and FL+LIF+RA), where miRNAs are hardly detectable (Fig. 4c).

Finally, in maGSCs if RA is added from Day 5 onwards $(\mathrm{Gel}+\mathrm{RA}$ from Day 5), miRNA levels at Day 10 are lower compared with Gel+RA condition, where RA was added from the beginning, contrasting with ESC R1 (Supplementary Fig. S3a).

\section{Members of miR-302 family are induced during first stages of in vitro differentiation}

The expression profiles of members of the 302-family were found to differ significantly from those of miR-290 family members (Fig. 5). In ESCs, the Gel condition has an extreme effect on the expression of miRNAs 302 at Day 5 (Fig. 5a). They become strongly up-regulated 


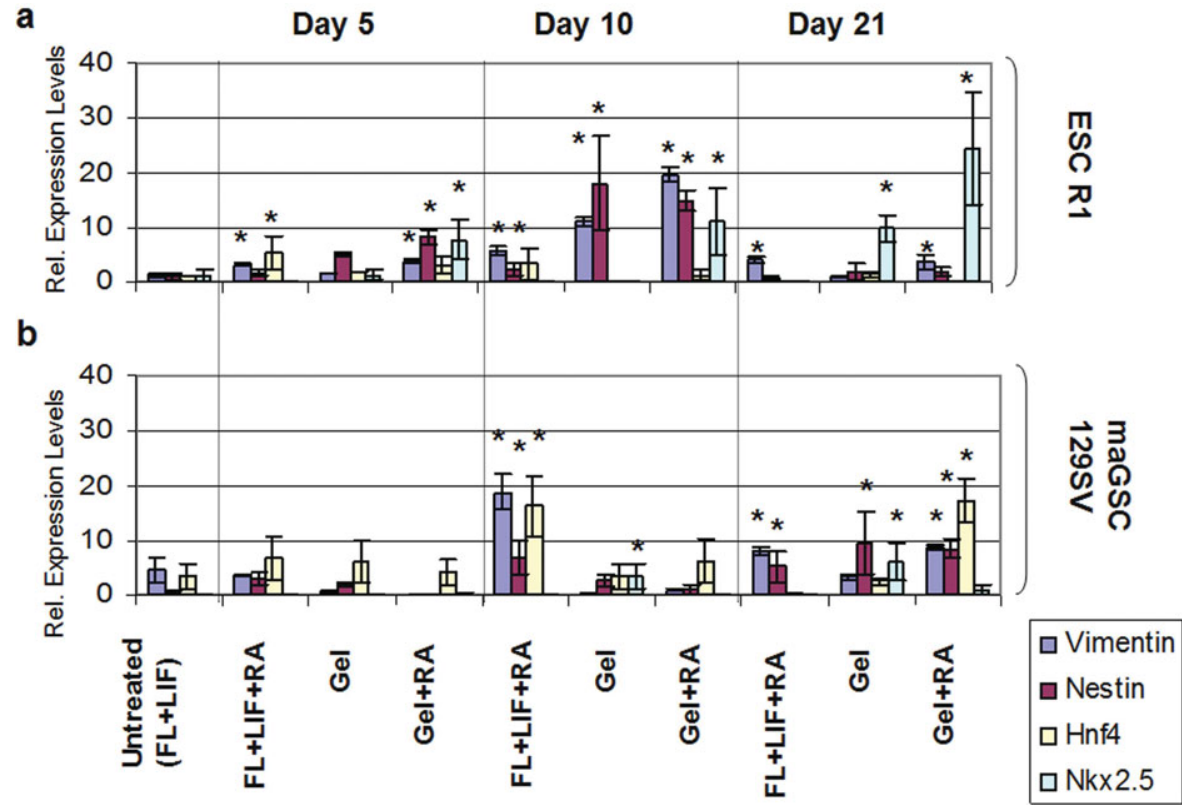

Figure 3: Expression profiles of differentiation markers (Vimentin, Nestin, Hnf4 and Nkx2.5) in ESCs and maGSCs from mouse strain 129/Sv (ESC R1 and maGSC 129SV) under different culture conditions after 5, 10 and 21 days in culture (vertical lines separate differentiation conditions of the same day from those of other days).

Asterisks indicate statistical significance for the comparison with untreated cells. All levels were normalized to endogenous control (Sdha) and calibrated to the value of untreated ESC R1 (ESC R1 FL+LIF at Day 5). (a) Expression profile in differentiating ESC R1 cells. (b) Expression profile in differentiating maGSC 129SV cells.

(20-100-fold increase). Their levels decrease rapidly after Day 5, but even at Day 10 and 21 of culture, the miRNA expression is still higher than that in untreated cells (Fig. 5b and c). In Gel+RA, miRNA levels increase temporally to levels higher than that in untreated cells around Day 10 (Fig. 5b). Then they decrease leading to expression levels lower than that in untreated cells at Day 21. In contrast, when RA is added from Day 5 onwards, such an increase at Day 10 does not occur (Supplementary Fig. S3b). miRNAs 302 also become up-regulated in maGSCs under Gel condition. During the culture period of 21 days, expression levels increase 10-30-fold (Fig. 5c). However, in the case of maGSCs, levels increase gradually at least until Day 21, and not only at Day 5 like in ESCs. In the other two conditions (Gel+RA and FL+LIF+RA), miRNA levels at Day 5 are higher than those in untreated cells (Fig. 5a) and become similar to them thereafter (Fig. $5 b$ and c). The increase from Day 5 to Day 10 observed in Gel is weaker (especially for mir-302b and d) when RA is added from Day 5 onwards (Gel+RA from Day 5), but miRNA levels in this condition are still higher compared with Gel+RA at the same day (Supplementary Fig. S3b).

\section{ESC-specific miRNAs are expressed in teratocarcinoma cell line $\mathbf{F 9}$}

In addition, we studied the expression of ESC-specific miRNAs in the teratocarcinoma cell line F9 [embryonic carcinoma cell (ECCs)] that was found to share many similarities with pluripotent cells (Andrews, 2002). As can be seen from Supplementary Fig. S1a and Fig. 1a, ECCs express the pluripotency markers Oct-4 and Sox-2 as well as the ESC-specific set of miRNA families 290 and 302. In the past, RA has been used to induce differentiation of these cells (Alonso et al., 1991). When ECCs are treated with $10^{-6} \mathrm{M}$ RA for 25 days, miR-290 and miR-291 levels decrease slightly, miR-292, miR-293 and miR-294 levels remain relatively stable and only miR-295 increases. In contrast, the levels of all miR-302 family members increase significantly (3-5-fold increase) (Fig. 6a). Both treated and untreated cells express the pluripotency markers Oct-4 and Sox-2 (Fig. 6b), but in treated cells an increase in the levels of differentiation markers Nestin and Hnf4 is observed (Fig. 6c).

\section{Discussion}

Previously, several authors have described a unique miRNA expression signature in mouse ESCs. Members of 290 and 302 miRNA families were previously classified as ESC-specific, since they are expressed only in undifferentiated ESCs. Expression of these miRNAs in ESC EBs is strongly down-regulated when ESCs are induced to differentiate and undetectable in adult organs (this, however, does not apply to miR-302 family during early in vitro differentiation as we show in the present study). Our results show that maGSCs share this unique miRNA expression signature with ESC lines. These miRNAs are also constantly expressed in maGSCs and down-regulated after long exposure to differentiation conditions. However, expression levels differed between maGSCs from different mouse strains, as well as between ESCs and maGSCs from the same mouse strain. A possible explanation for this, apart from the different genetic background, could be the different passage number of the cell lines tested. As shown in Figs 1b, 4 and 5, even under standard ESC culture conditions miRNA expression levels vary between different passage numbers (which, for example, in the case of miR-293 demonstrates an increase in expression levels of more than 50\% in untreated ESCs at Day 21, Fig. 4c, compared with untreated ESCs at Day 5, Fig. 4a). maGSCs Stra8 was the maGSC line of the highest passage number used in this study, which could explain the different miRNA expression pattern compared with the other maGSC lines that are of lower passage number. For this reason in all other experiments, cell lines of the same passage number and the same mouse strain $(129 / \mathrm{Sv})$ were used to eliminate this effect. In this case, when cell lines of the same passage number are used, maGSCs $129 \mathrm{SV}$ seem to express these miRNAs in lower levels than ESCs from the 


\section{amiR-290 amiR-291 amiR-292 amiR-293 amiR-294 amiR-295}

\section{a}

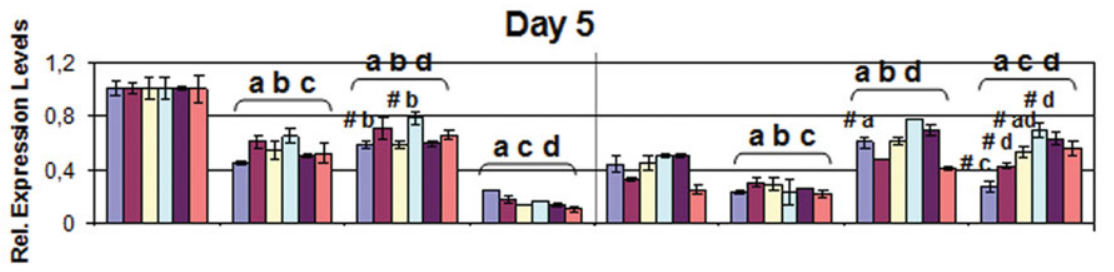

b

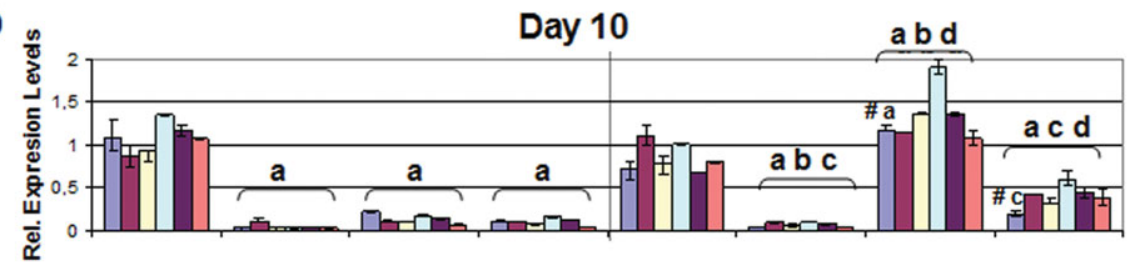

C

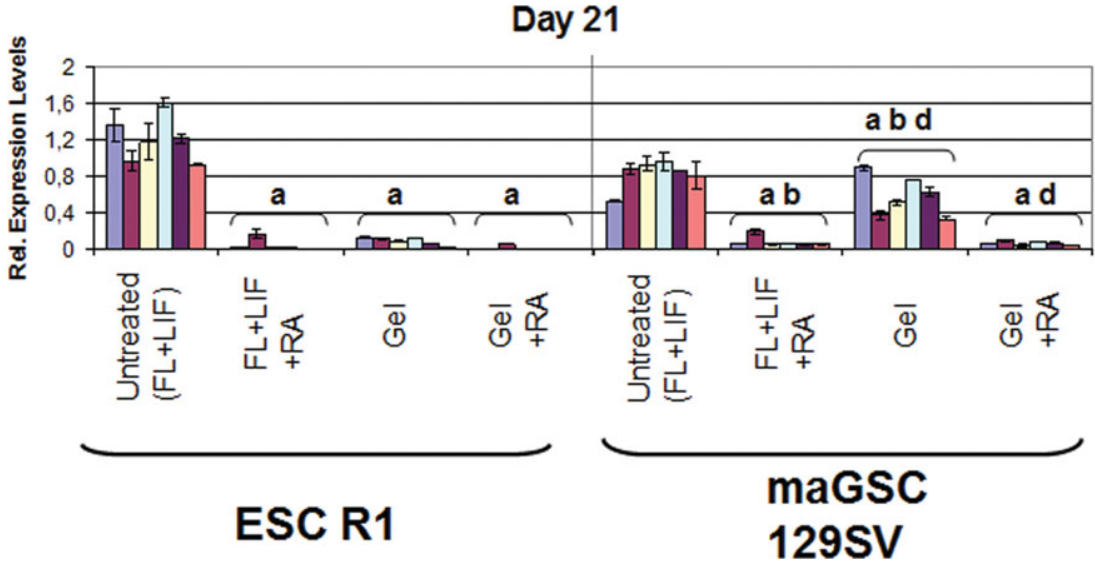

Figure 4: Expression profiles of members of the miRNA 290 family in ESCs and maGSCs from mouse strain 129/Sv (ESC R1 and maGSC 129SV) under different culture conditions after 5, 10 and 21 days (Fig. $4 \mathrm{a}, \mathrm{b}$ and c, respectively) in culture.

$\mathrm{a}, \mathrm{b}, \mathrm{c}$ and d depicted in each subfigure above the different conditions indicate statistical significance for the following pair comparisons, a: comparison of each differentiation condition with untreated cells, b: comparison between FL+LIF+RA and GEL, c: comparison between FL+LIF+RA and GEL+RA, d: comparison between GEL and GEL+RA. Combination of two or three letters indicates statistical significance for more than one comparison. For example, a b d above GEL condition refers to comparison of this condition with all other conditions. The letters apply to all miRNAs of each condition with the exception of these miRNAs with a \# above them, which indicates no statistical significance for the corresponding miRNA and the corresponding comparison. For example \#a above miRNA-290 in one condition means no statistical significance for miR-290 in this condition compared with untreated cells. The line that cuts the diagram into two parts separates ESCs (left) from maGSCs (right). All levels were normalized to endogenous control (RNU6B) and calibrated to the value of untreated ESC R1 (FL+LIF) at Day 5 (Fig. 4a).

same background (ESC R1). We also detected these miRNAs in high levels in F9 teratocarcinoma cells, which have also been shown to be pluripotent (Andrews, 2002). Thus, it is shown that these miRNAs generally characterize pluripotent cells. However, in contrast to F9 cells, proliferation and expression of these markers in maGSCs are restricted to standard ESC culture conditions. This is an important similarity between ESCs and maGSCs that distinguishes them from ECCs.

Several authors have studied expression profiles of members of miRNA families 290 and 302 during ESC differentiation. They found a negative correlation between their expression levels and differentiation over time (Houbaviy et al., 2003; Strauss et al., 2006; Chen et al., 2007). Because ESCs and maGSCs share great similarities in pluripotency (Kanatsu-Shinohara et al., 2004, 2008; Guan et al., 2006; Seandel et al., 2007) (Supplementary Figs S1 and S2), we decided to study the profiles of both miRNA families during differentiation of both cell types. Owing to the high number of differentiation strategies so far described, we concentrated on the most important factors that prevent or induce differentiation in ESC culture, namely LIF and RA, respectively (Rohwedel et al., 1999; Rao, 2004; Kurosawa, 2007; Tighe and Gudas, 2004; Liu et al., 2007). Loss of pluripotent state of the cells tested was evaluated by determining expression levels of well-known pluripotency markers as well as differentiation markers like Nestin (neural stem cell marker) (Lin et al., 1995; Lendahl, 1997; Wiese et al., 2004), Vimentin (early neuro-ectoderm formation and cells of mesodermal origin) (Franke et al., 1982; Boisseau and Simonneau, 1989; ColucciGuyon et al., 1999), Hnf4 (endoderm) (Taraviras et al., 1994; Duncan et al., 1997) and Nkx2.5 (early embryo heart formation) (Liberatore et al., 2002).

The observation of other authors that the members of miRNA family 290 are down-regulated in ESCs during differentiation is supported by our results and a down-regulation was found to be realized during maGSCs differentiation. However, in maGSCs under Gel and 


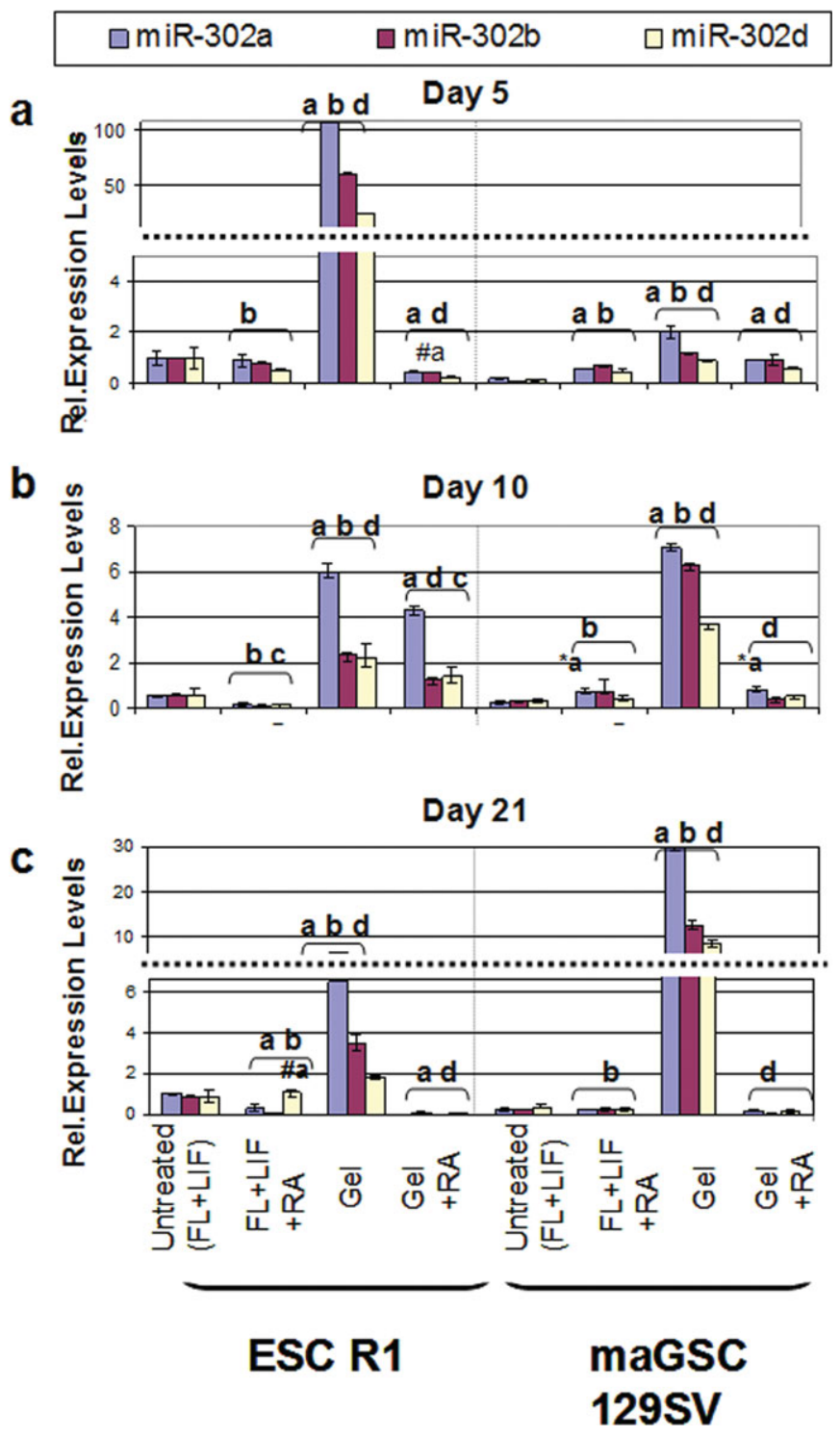

Figure 5: Expression profiles of members of the miRNA 302 family in ESCs and maGSCs from mouse strain 129/Sv (ESC R1 and maGSC 129SV) under different culture conditions after 5, 10 and 21 days in culture (Fig. 5a, b and c, respectively).

The line that cuts the diagram into two parts separates ESCs (left) from maGSCs (right). All levels were normalized to endogenous control (RNU6B) and calibrated to the value of untreated ESC R1 (FL+LIF) at Day 5 (Fig. 5a). For symbols indicating statistical significance, see Fig. 4. Additionally, asterisk above miR-302a in Fig. 5b indicates statistical significance only for this miRNA for the comparison of the respective condition with untreated cells.

Gel+RA condition, miRNA levels remain high for a longer period than in ESCs or even increase transiently (Gel condition). These differences seem to correlate with the differences in the differentiation status of these cells. Under Gel condition, Nestin and Vimentin are up-regulated earlier in ESCs, whereas Oct-4 expression decreases later in maGSCs. In addition, FL+LIF+RA, which is the only condition in maGSCs at Day 10 with a significant increase in most differentiation markers, is characterized by a strong down-regulation of miRNA levels. Since the expression profile of Oct-4 corresponds to that of members of miRNA family 290 in both ESCs and maGSCs, our results indicate that expression of these miRNAs is more connected with maintenance of pluripotency than with differentiation.
Chen et al. have studied expression of members of the miRNA 302 family in ESCs at Days 3, 6 and 9 during EB formation in the absence of LIF. They found that these miRNAs are negatively correlated to differentiation time (Chen et al., 2007). This expression profile in ESCs is different from that we obtained in our study, since during the first 5 days of differentiation under Gel condition, all members of miRNA family 302 are strongly up-regulated. Transient up-regulation of these miRNAs in ESCs is also observed in the presence of RA, although not so strongly as in Gel condition. Expression profiles of these miRNAs in maGSCs demonstrate similarities and differences compared with ESCs. Under Gel condition, strong up-regulation of miRNA levels is also observed, and addition of RA was found to result also in an up-regulation of these miRNAs. However, in maGSCs up-regulation under Gel condition takes place slowly. At Day 21, miRNA levels in maGSCs depict 10-30-fold increase, whereas in ESCs 20-100-fold increase is reached already at Day 5. This gradual increase in maGSCs correlates to the differences in differentiation status between ESCs and maGSCs mentioned above. In addition, the increase under Gel+RA condition in maGSCs occurs at Day 5 and not at Day 10 as in ESCs.

Since the expression profile of Oct-4 does not correspond to that of 302 miR-family members, our results suggest that these miRNAs are more connected with response of pluripotent cells to differentiation than with the undifferentiated state itself. This is in contradiction to the observation that, even in undifferentiated cells, miRNAs 302 are present but can be explained by the observation that cultures of pluripotent cells contain spontaneously differentiated cells (Houbaviy et al., 2003).

The connection of members of miRNA family 290 with pluripotency and that of members of miRNA family 302 with the process of differentiation is further supported by our miRNA analysis in ECCs and by comparing Gel+RA condition with Gel+RA from Day 5 condition. In ECCs (Fig. 6), where addition of RA is followed by an increase in Nestin and Hnf4 expression, miR-302 family is up-regulated. At the same time, treated cells retain expression of miR-290 family as they do for Oct-4. When Gel+RA and Gel+RA from Day 5 conditions are compared, high levels of miR-290 family are connected with low levels of miR-302 family and most of differentiation markers (with the exception of Hnf4) and vice versa. This connection is also supported by the findings of Tang et al. They have shown that the miRNA 290 family belongs to the most significant miRNAs strongly up-regulated in early mouse embryogenesis from 2-cell stage onwards (Tang et al., 2007). This is exactly the stage when Oct-4 expression increases (Schöler et al., 1989). In contrast, miRNA family 302 does not show significant expression changes. However, the exact correlation of miR-302 expression with a specific lineage commitment requires differentiation strategies that are beyond the scope of this study and the simple differentiation model used here. In addition, it was recently shown that miRNAs of the miR-290 family control de novo DNA methylation through regulation of transcriptional repressors in mouse ESCs (Sinkkonen et al., 2008) which implies that differences observed during differentiation between ESCs and maGSCs may be connected with the differences observed in the miRNA level.

miRNAs are believed to play a crucial role in development by regulating expression of hundreds of genes simultaneously. Members of miRNA families 290 and 302, which were previously classified as ESC specific, are candidates for such a role in pluripotent stem cells and not only in mouse, since they have close homologues in human ESCs with similar expression profile during differentiation. Our results support further the connection of miR-290 family with maintenance of pluripotency and provide indirect evidence for a possible role of members of miR-302 family during first stages of in vitro 
a

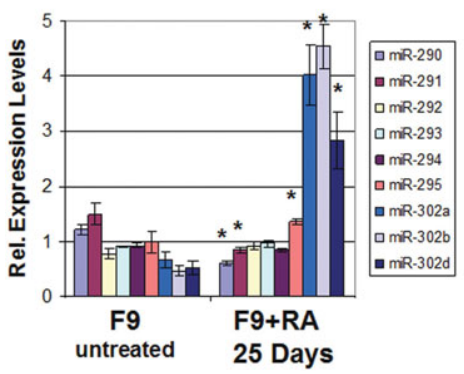

b

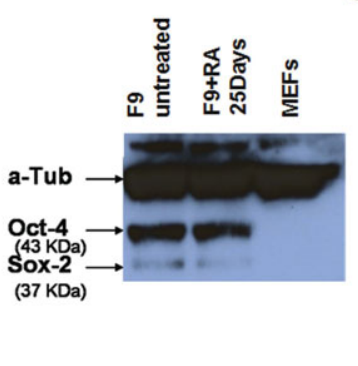

C

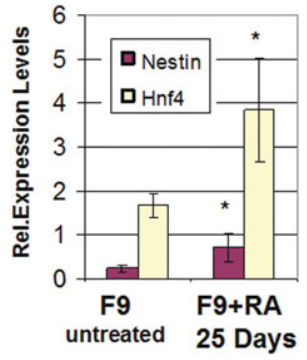

Figure 6: Expression of ESC-specific miRNAs and of pluripotency and differentiation markers in F9 cells.

All levels were calibrated to the value of untreated ESC R1 (FL+LIF) at Day 5 (Figs 3, 4 and 5). (a) Expression profiles of members of miRNA families 290 and 302 in untreated F9 cells and in those treated with RA for 25 days. Asterisk depicts statistical significance for this miRNA in comparison with untreated. (b) Expression analysis by western blotting of pluripotency markers Oct-4 and Sox-2 in F9 cells without and after treatment with RA for 25 days. MEFs were used as negative control and a-Tubulin served as loading control. (c) Expression of Nestin and Hnf4 in untreated and treated cells. Asterisk depicts statistical significance in comparison with untreated.

differentiation of pluripotent cells. Moreover, detection of these miRNAs in maGSCs is consistent with the ESC-like nature of maGSCs and their potential as an alternative source of pluripotent cells.

\section{Author's contribution}

A.Z.: Conception and design, provision of study material, collection and assembly of data, data analysis and interpretation, manuscript writing.

J.N.: Conception and design, provision of study material, manuscript writing.

N.D.: Data analysis and interpretation.

U.Z.: Manuscript writing, final approval of manuscript.

H.H.: Collection and assembly of data.

K.G.: Provision of study material, final approval of manuscript.

G.H.: Provision of study material, final approval of manuscript.

K.N.: Provision of study material, final approval of manuscript.

W.E.: Conception and design, financial support, administrative support, manuscript writing, final approval of manuscript.

\section{Funding}

This work was supported by the German Research Foundation (Deutsche Forschungsgemeinschaft: SPP 1356; EN 84/22-1, ZE 442/4-1).

\section{Acknowledgements}

We would like to thank Dr Stanton (Singapore) for providing the Zfp-206 antibody. We also thank Dr A. Zibat for technical assistance with real-time PCR and Britta Kaltwasser for cell culture work.

\section{References}

Alonso A, Breuer B, Steuer B, Fischer J. The F9-EC cell line as a model for the analysis of differentiation. Int J Dev Biol 1991;35:389-397.

Andrews PW. From teratocarcinomas to embryonic stem cells. Philos Trans $R$ Soc Lond B Biol Sci 2002;357:405-417.

Bartel DP. MicroRNAs: Genomics, biogenesis, mechanism, and function. Cell 2004;116:281-297.

Bartel DP, Chen CH. Micromanagers of gene expression: the potentially widespread influence of metazoan microRNAs. Nat Rev Genet 2004;5: 396-400.

Boisseau S, Simonneau M. Mammalian neuronal differentiation: early expression of a neuronal phenotype from mouse neural crest cells in a chemically defined culture medium. Development 1989;106:665-674.

Buitrago W, Roop DR. Oct-4: The almighty POUripotent regulator? J Invest Dermatol 2007;127:260-262.
Chen C, Ridzon D, Lee CT, Blake J, Sun Y, Strauss WM. Defining embryonic stem cell identity using differentiation-related microRNAs and their potential targets. Mamm Genome 2007;18:316-327.

Cheng J, Dutra A, Takesono A, Garrett-Beal L, Schwartzberg PL. Improved generation of $\mathrm{C} 57 \mathrm{BL} / 6 \mathrm{~J}$ mouse embryonic stem cells in a defined serum-free media. Genesis 2004;39:100-104.

Colucci-Guyon E, Gimenez Y, Ribotta M, Maurice T, Babinet C, Privat A Cerebellar defect and impaired motor coordination in mice lacking vimentin. Glia 1999;25:33-43.

Duncan SA, Nagy A, Chan W. Murine gastrulation requires HNF-4 regulated gene expression in the visceral endoderm: tetraploid rescue of Hnf-4(-/-) embryos. Development 1997;124:279-287.

Franke WW, Grund C, Kuhn C, Jackson BW, Illmensee K. Formation of cytoskeletal elements during mouse embryogenesis. III. Primary mesenchymal cells and the first appearance of vimentin filaments. Differentiation 1982;23:43-59.

Guan K, Nayernia K, Maier LS, Wagner S, Dressel R, Lee JH, Nolte J, Wolf F, Li M, Engel W et al. Pluripotency of spermatogonial stem cells from adult mouse testis. Nature 2006;440:1199-1203.

Houbaviy H, Murray M, Sharp P. Embryonic stem cell-specific microRNAs. Dev Cell 2003;5:351-358.

Houbaviy H, Dennis L, Jaenisch R, Sharp PA. Characterization of a highly variable eutherian microRNA gene. RNA 2005;11:1245-1257.

Izadyar F, Pau F, Marh J, Slepko N, Wang T, Gonzalez R, Ramos T, Howerton K, Sayre C, Silva F. Generation of multipotent cell lines from a distinct population of male germ line stem cells. Reproduction 2008; 135:771-784.

Johnson SM, Grosshans H, Shingara J. RAS is regulated by the let-7 microRNA family. Cell 2005;120:635-647.

Kanatsu-Shinohara M, Inoue K, Lee J, Yoshimoto M, Ogonuki N, Miki H, Baba S, Kato T, Kazuki Y, Toyokuni S et al. Generation of pluripotent stem cells from neonatal mouse testis. Cell 2004;119:1001-1012.

Kanatsu-Shinohara M, Lee J, Inoue K, Ogonuki N, Miki H, Toyokuni S, ikawa M, Tomoyuki N, Ogura A, Shinohara T. Pluripotency of a single spermatogonial stem cell in mice. Biol Reprod 2008;78:681-687.

Kurosawa H. Methods for inducing embryoid body formation: in vitro differentiation system of embryonic stem cells. J Biosci Bioeng 2007;103: 389-398.

Lendahl U. Transgenic analysis of central nervous system development and regeneration. Acta Anaesthesiol Scand Suppl 1997;110:116-118.

Liberatore CM, Searcy-Schrick RD, Vincent EB, Yutzey KE. Nkx-2.5 gene induction in mice is mediated by a Smad consensus regulatory region. Dev Biol 2002;244:243-256.

Lin RC, Matesie DF, Marvin M, McKay RD, Bruestle O. Re-expression of the intermediate filament nestin in reactive astrocytes. Neurobiol Dis 1995;2: $79-85$.

Liu N, Lu M, Tian X, Han Z. Molecular mechanisms involved in self-renewal and pluripotency of embryonic stem cells. J Cell Physiol 2007;211:279-286.

Masui S, Nakatake Y, Toyooka Y, Shimosato D, Yagi R, Takahashi K, Okochi $\mathrm{H}$, Okuda A, Matoba R, Sharov AA et al. Pluripotency governed by Sox2 via regulation of Oct3/4 expression in mouse embryonic stem cells. Nat Cell Biol 2007:9:625-635. 
Nayernia K, Li M, Jaroszynski L, Khusainov R, Wulf G, Schwandt I, Korabiowska M, Michelmann HW, Meinhardt A, Engel W. Stem cell based therapeutical approach of male infertility by teratocarcinoma derived germ cells. Hum Mol Genet 2004;13:1451-1460.

Pan G, Thomson JA. Nanog and transcriptional networks in embryonic stem cell pluripotency. Cell Res 2007;17:42-49.

Rao M. Conserved and divergent paths that regulate self-renewal in mouse and human embryonic stem cells. Dev Biol 2004;275:269-286.

Rodda DJ, Chew JL, Lim LH, Loh YH, Wang B, Ng HH, Robson P. Transcriptional regulation of Nanog by Oct4 and Sox2. J Biol Chem 2005;280:24731-24737.

Rohwedel J, Guan K, Wobus A. Induction of cellular differentiation by retinoic acid in vitro. Cells tissues Organs 1999;165:190-202.

Schöler HR, Hatzopoulos AK, Balling R, Suzuki N, Gruss P. A family of octamer-specific proteins present during mouse embryogenesis: evidence for germline-specific expression of an Oct factor. EMBO J 1989;8:2543-2550.

Seandel M, James D, Shmelkov SV, Falciatori I, Kim J, Chavala S, Scherr DS, Zhang F, Torres R, Gale NW et al. Generation of functional multipotent adult stem cells from GPR125+ germline progenitors. Nature 2007;449: $346-350$.

Sinkkonen L, Hugenschmidt T, Berninger P, Gaidatzis D, Mohn F, Artus-Revel CG, Zavolan M, Svoboda P, Filipowicz W. MicroRNAs control de novo DNA methylation through regulation of transcriptional repressors in mouse embryonic stem cells. Nat Struct Mol Biol 2008;15:259-267.

Strauss W, Chen C, Lee CT, Ridzon D. Nonrestrictive developmental regulation of microRNA gene expression. Mamm Genome 2006;17: 833-840.

Suh MR, Lee Y, Kim JY, Kim SK, Moon SH, Lee JY, Cha KY, Chung HM, Yoon HS, Moon SY et al. Human embryonic stem cells express a unique set of microRNAs. Dev Biol 2004;270:488-498.
Tang F, Kaneda M, O'Carroll D, Hajkova P, Barton SC, Sun YA, Lee C, Tarakhovsky A, Lao K, Surani MA. Maternal microRNAs are essential for mouse zygotic development. Genes Dev 2007;21:644-648.

Taraviras S, Monaghan AP, Schütz G, Kelsey G. Characterization of the mouse HNF-4 gene and its expression during mouse embryogenesis. Mech Dev 1994;48:67-79.

Tighe A, Gudas LJ. Retinoic acid inhibits leukemia inhibitory factor signaling pathways in mouse embryonic stem cells. J Cell Physiol 2004; 198: $223-229$.

Wang ZX, Kueh JL, Teh CH, Rossbach M, Lim L, Li P, Wong KY, Lufkin T, Robson P, Stanton LW. Zfp206 is a transcription factor that controls pluripotency of embryonic stem cells. Stem Cells 2007a;25:2173-2182.

Wang ZX, Teh Ch, Kueh JL, Lufkin T, Robson P, Stanton LW. Oct4 and Sox2 directly regulate expression of another pluripotency transcription factor, Zfp206, in embryonic stem cells. J Biol Chem 2007b;282: 12822-12830.

Wiese C, Rolletschek A, Kania G, Blyszczuk P, Tarasov KV, Tarasova Y, Wersto RP, Boheler KR, Wobus AM. Nestin expression-a property of multi-lineage progenitor cells? Cell Mol Life Sci 2004;61:2510-2522.

Wurst W, Joyner AL. Production of targeted embryonic stem cell clones. In: Joyner AL (ed). Gene Targeting: A Practical Approach. Oxford, UK: IRL Press, 1993,33-61.

Zhang J, Tam WL, Tong GQ, Wu Q, Chan HY, Soh BS, Lou Y, Yang J, Ma Y, Chai L et al. Sall4 modulates embryonic stem cell pluripotency and early embryonic development by the transcriptional regulation of Pou5f1. Nat Cell Biol 2006;8:1114-1123.

Submitted on March 29, 2008; resubmitted on July 24, 2008; accepted on August 6, 2008 


\section{LEGENDS TO FIGURES IN SUPPLEMENTARY DATA}

Figure S1. Expression of pluripotency markers in different ESC lines, maGSC lines, F9 cells, mouse testis and MEFs. (a) Oct-4 and Sox-2; the same membrane was used for both antibodies. The reason why no Oct-4 was detected in the testis sample although previous reports demonstrate Oct-4 immunoreactivity by histochemical methods from embryonic through adult testes could be attributed to the fact that spermatogonial stem cells constitute only $0.03 \%$ of all germ cells making it difficult to detect by Western analyis. (b) Zfp-206 and Sall-4. a-Tubulin served as loading control. Cal = calibrator

Figure S2. Expression of pluripotency markers in ESCs and maGSCs from 129/Sv background (ESC R1 and maGSC 129SV) and after culture. Cells were cultured for 35 days under standard ESC culture conditions (from passage 15 to passage 25 ) and in $0.1 \%$ gelatine coated flasks without LIF but with RA (Gel+RA). (a) Western Blotting for Oct-4 and Sox-2. Levels of Oct-4 and Sox2 were quantified densitometrically. Asterisks indicate statistical significance in comparison with untreated cells. (b) Immunostaining for SSEA-1. The ES cell characterization kit (chemicon) was used to immunostain for SSEA-1 with Cy3-conjugated anti-rabbit 1gG secondary antibody.

Figure S3. Comparison of expression levels of members of miRNAs and differentiation markers at day 10 in ESCs and maGSCs from mouse strain 129/Sv (ESC R1 and maGSC 129SV) between Gel+RA (cells cultured in Gel coated flasks with addition of RA from the beginning) and Gel+RA from day 5 (cells cultured in Gel coated flasks with addition of RA from day 5) conditions. The 
line that cuts the diagram in two parts, separates ESCs (left) from maGSCs (right). All miRNA and mRNA levels were normalised to endogenous control (RNU6B and Sdha respectively) and calibrated to the value of untreated ESC R1 (FL+LIF) at day 5. Asterisk indicates statistical significance comparing with Gel +RA from day 0 condition. \# indicates no statistical significance (a) Expression levels of miR-290 family. (b) Expression levels of miR-302 family . (c) Expression levels of Vimentin,Nestin, Hnf4 and Nkx2.5 
a

\section{$\begin{array}{llllllll}1 & 2 & 3 & 4 & 5 & 6 & 7 & 8\end{array}$}
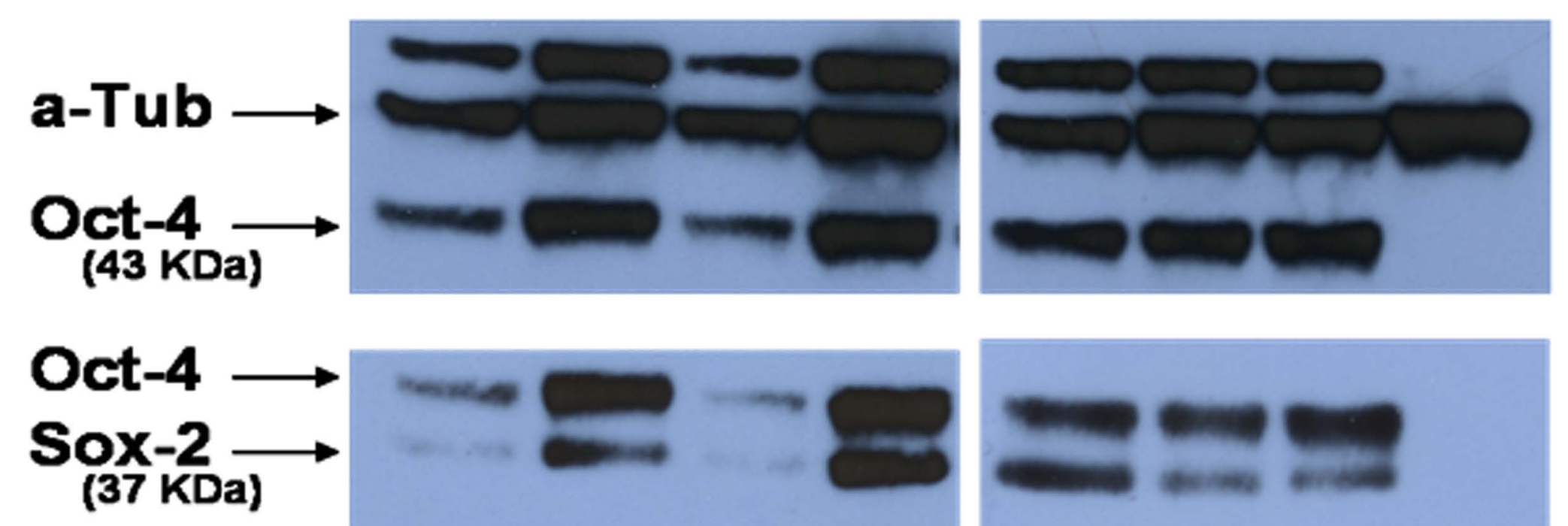
(37 KDa)
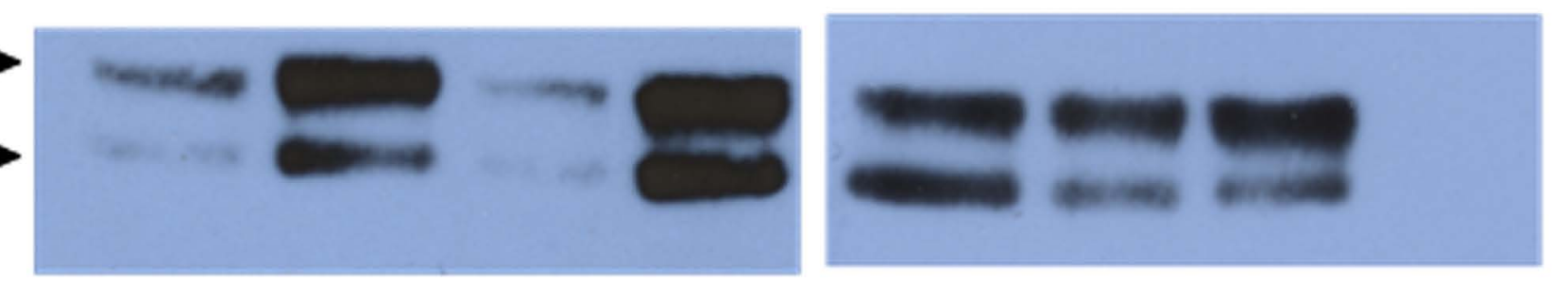

1. ESC R1

2. maGSC 129SV

3. ESC Stra8

4. maGSC Stra8

5. maGSC FVB

6. maGSC C57BL

7. $F 9$

8. testis

b

$\begin{array}{llllll}1 & 2 & 3 & 4 & 5 & 6\end{array}$

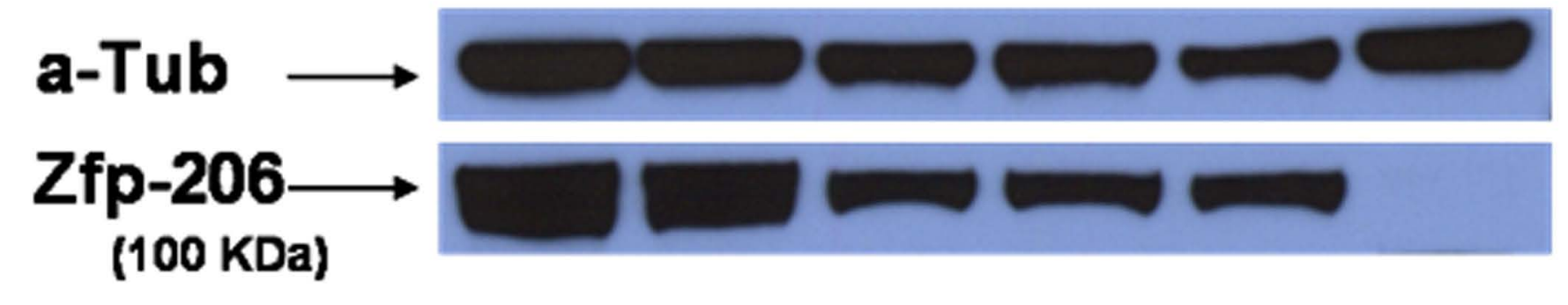

1. ESC R1

2. maGSC Stra8

3. maGSC FVB

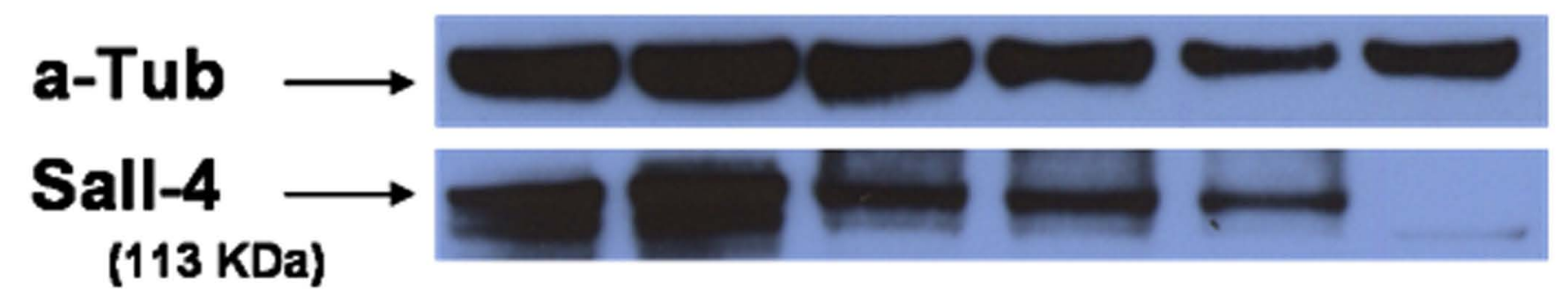

4. maGSC C57BL

5. maGSC 129SV

6. MEFS 
a
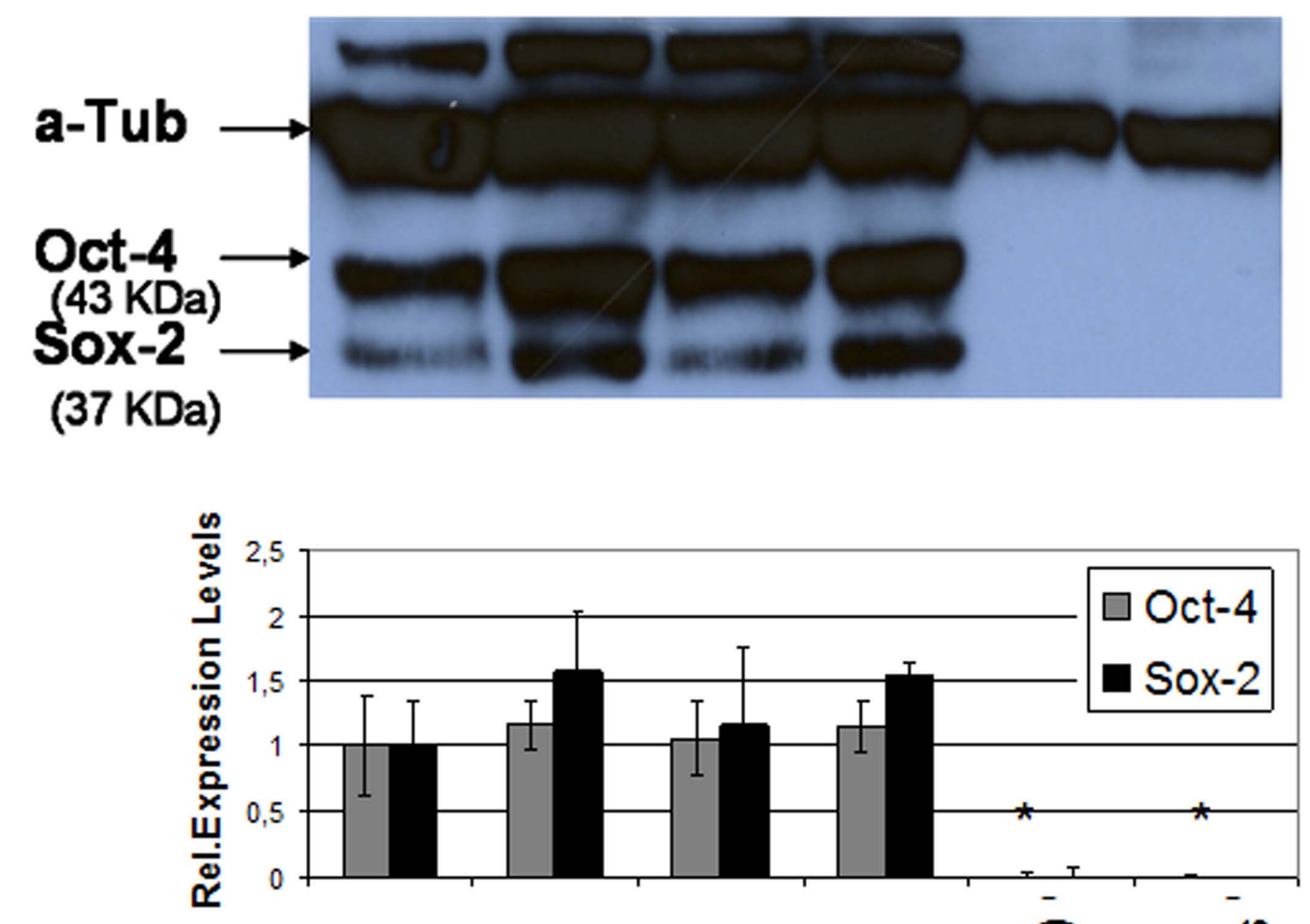

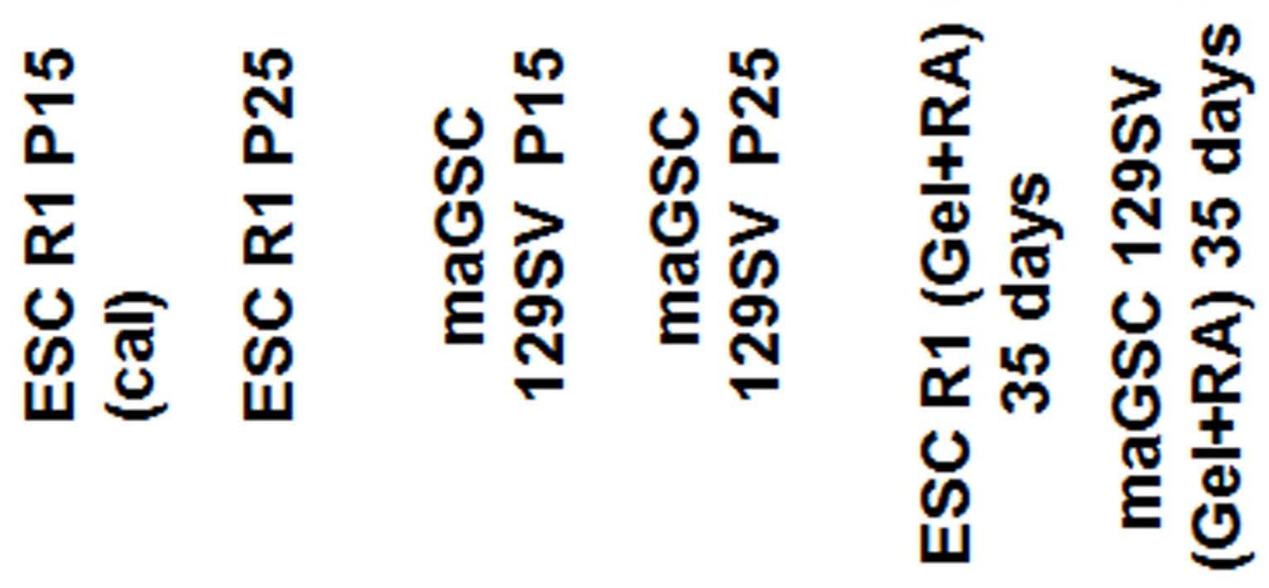
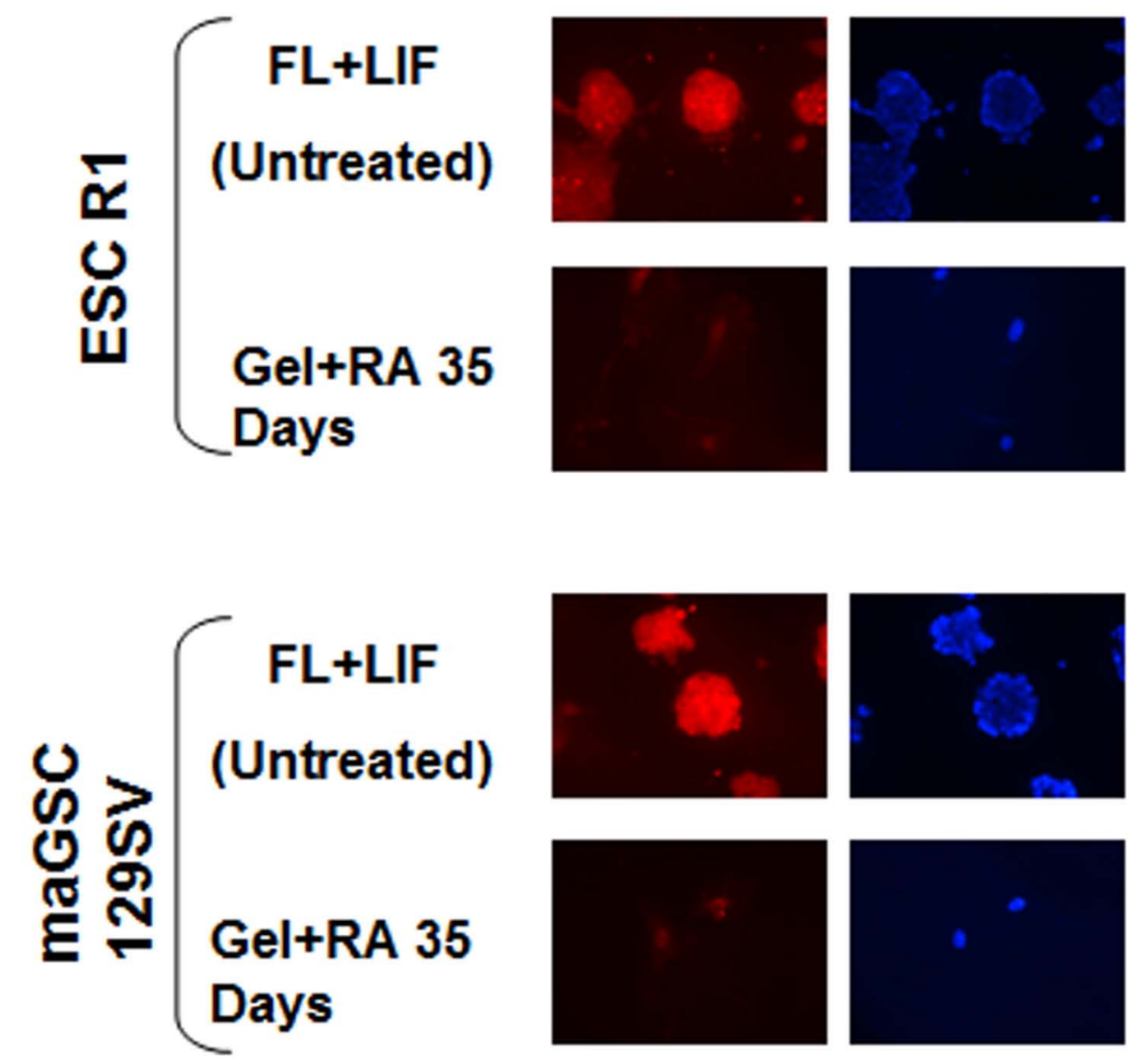

Cy-3
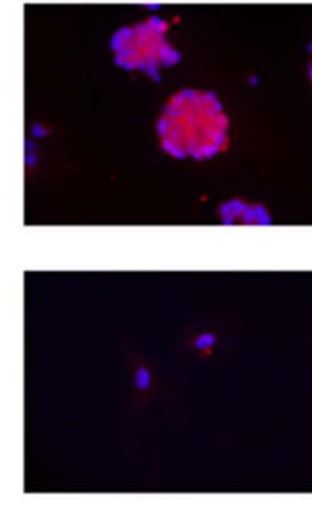

DAPI Overlay 
a

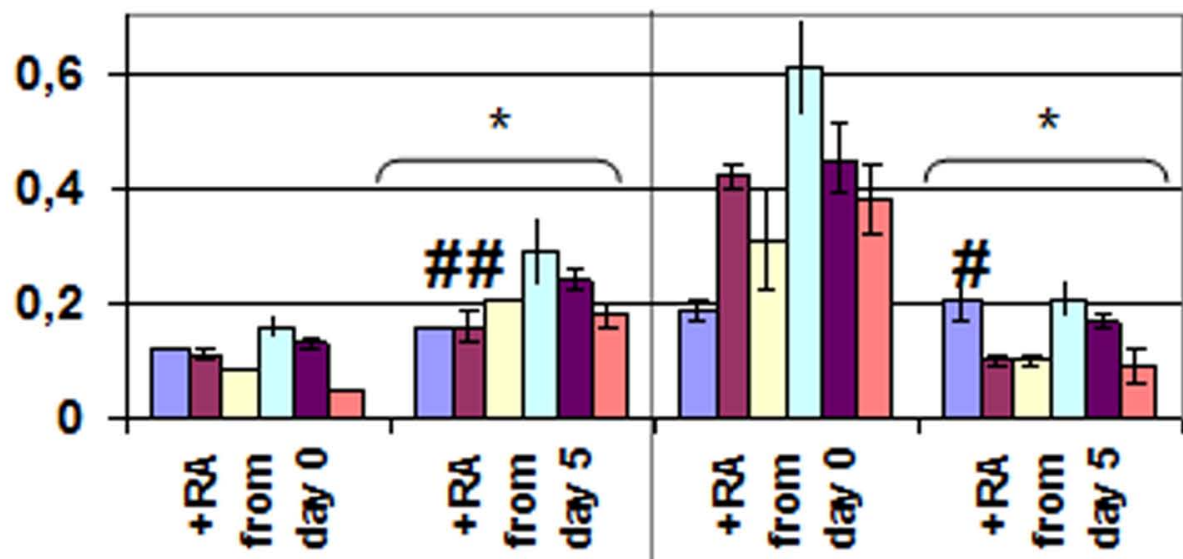

$|\square \mathrm{miR}-290|$

口 mir-291

c miR-292

口 miR-293

- miR-294

口 miR-295

b

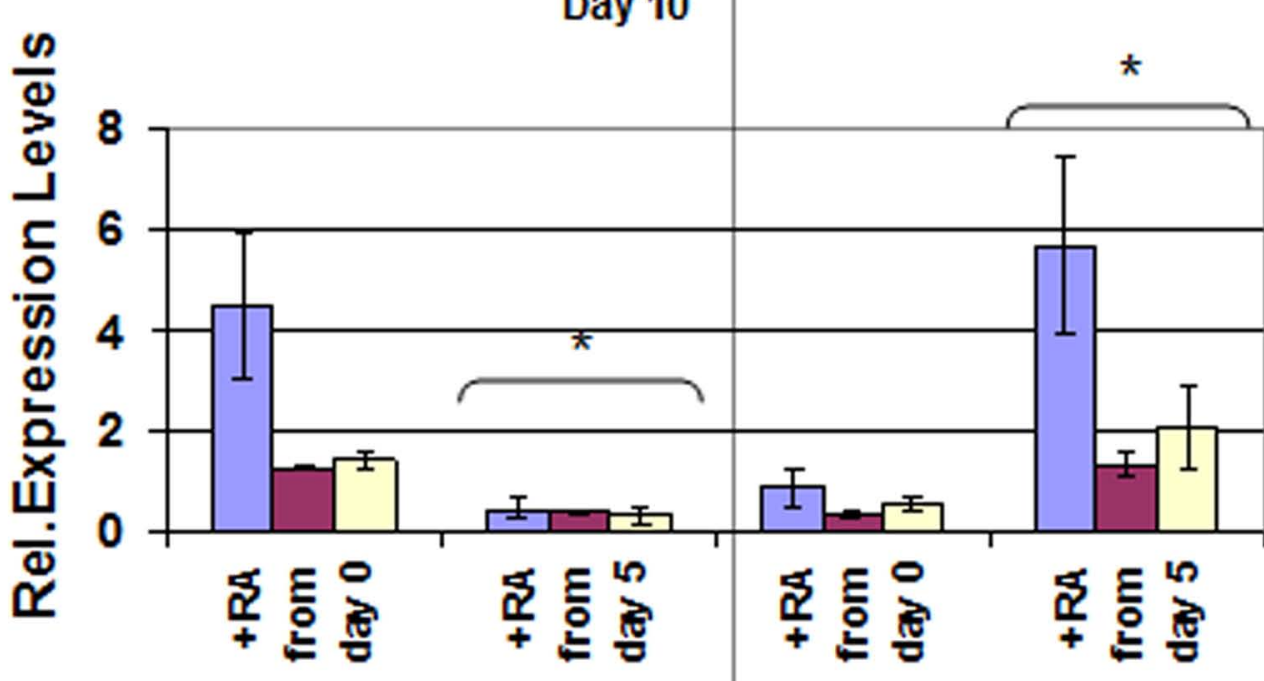

$\square \mathrm{miR}-302 \mathrm{a}$

a miR-302b

$\square \mathrm{miR}-302 \mathrm{~d}$

C

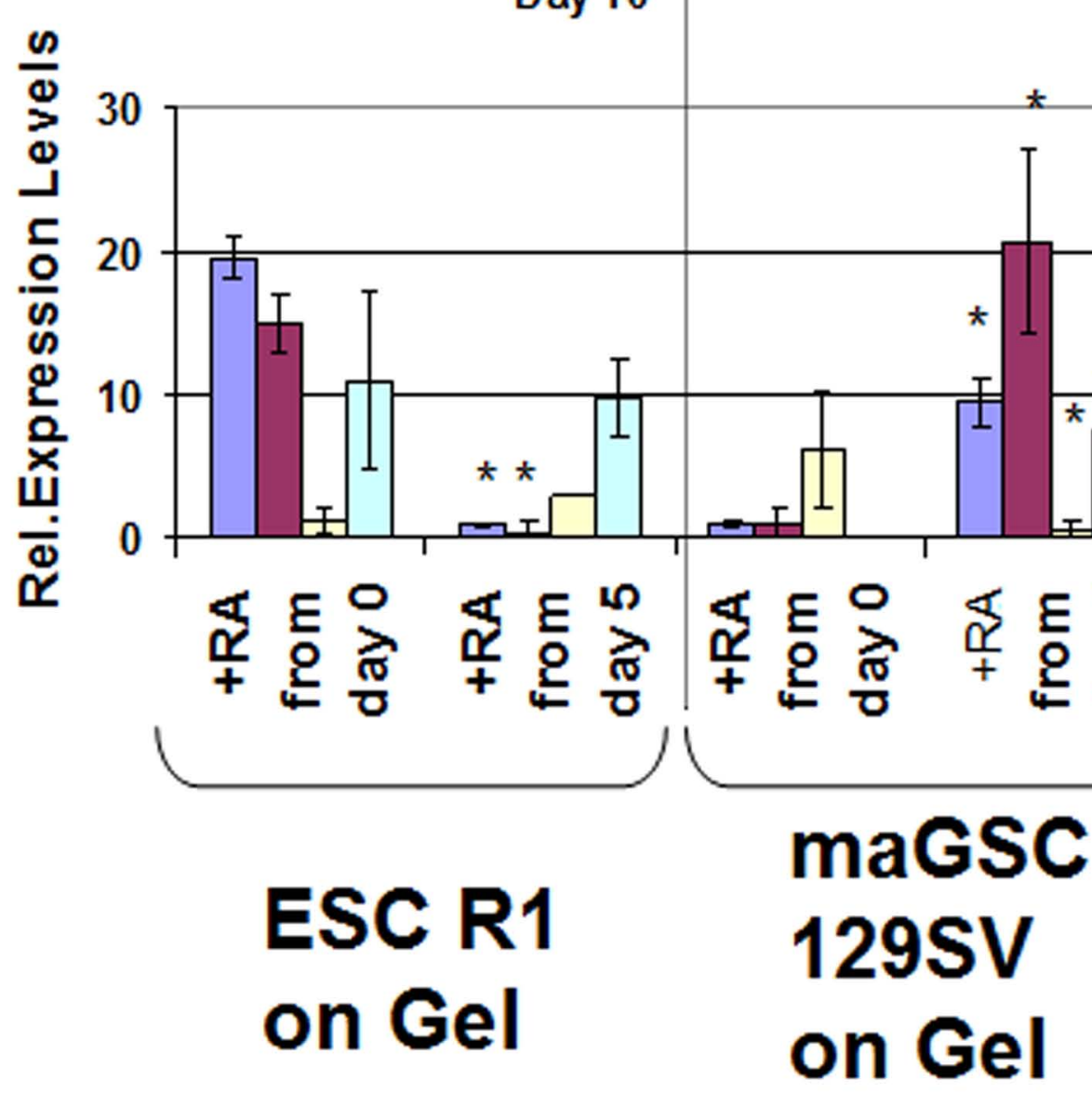

$\square$ Vimentin

$\square$ Nestin

$\square \mathrm{Hnf4}$

$\square \mathrm{Nkx} 2.5$

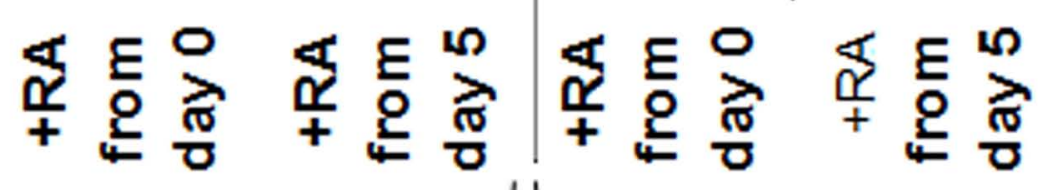

maGSC

129SV

on Gel 
Supplementary Table 1:

Primers used in qRT-PCR of Vimentin, Hnf4, Nestin, Nkx2.5 and Sdha

\begin{tabular}{|l|l|}
\hline Primer name & Sequence \\
\hline mVimentin-F & 5-TGCAGTCATTCAGACAGGATGT-3 \\
\hline mVimentin-R & 5-ATCTCTTCATCGTGCAGTTTCTTC-3 \\
\hline mHNF4-F & 5-CCACATGTACTCCTGCAGGTTTAG-3 \\
\hline mHNF4-R & 5-CGCTCATTTTGGACAGCTTC-3 \\
\hline mNestin-F & 5-CTGCAGGCCACTGAAAAGTT-3 \\
\hline mNestin-R & 5-ATTAGGCAAGGGGGAAGAGA-3 \\
\hline mNkx2.5-F & 5-CCCAAGTGCTCTCCTGCTTTCC-3 \\
\hline mNkx2.5-R & 5-GTCCAGCTCCACTGCCTTCTG-3 \\
\hline mSdha-F & 5-GCTTGCGAGCTGCATTTGG-3 \\
\hline mSdha-R & 5-CATCTCCAGTTGTCCTCTTCCA-3 \\
\hline
\end{tabular}




\section{Acknowledgements}

I am very grateful to several people for their help and support. This work owes the most to Prof W. Engel, Director of the Institute of Human Genetics in Goettingen and Group leader of the research group I performed this study. Without Prof Engel's support and mentorship this work would not have existed.

I also owe a lot to my colleague and friend, Nadja Drusenheimer. Nadja taught me many of the molecular biology techniques I know and helped me with many useful comments especially related to protein expression analysis and cell culture. I am also grateful to my lab colleagues that have contributed actively to this study: Mr Hiroki Hada helped me with the performance of the Western blotting results, while Mrs Pantazi established the Real-time PCRs for the different differentiation markers and helped with the validation of these data.

I have also to thank all current and previous members of Engel and Hasenfuss Groups that have contributted to derivation and characterization of mouse maGSCs. Special thanks also go to Britta Kaltwasser for providing some of the ESC culture media needed during this study. I am grateful to Dr. Arne Zibat for teaching me the Real-time PCR and to Dr. Silke Kaulfuss for supporting me in the use of microscope and densitometry software, as well as for her many useful comments on interpretation of Real-time and Western blotting results. Thanks also go to Dr. Pawel Grzmil for his support with the statistical analysis of the results.

I thank Mrs Jessica Nolte, Mr Krishna Pantakani, Mr Odgerel

Oidovsambuu, Mrs Aleksandra Kata and Mrs Ilona Paprotta for their comments and their invaluable everyday help throughout this study 
concerning materials and methods employed in this work and $\mathrm{Mr}$ Kazimierz Turek for support with computer software and hardware. Their help has been very much appreciated. Many thanks go to Qiagen technical support staff for helping with establishment of miRNA analysis. I would also like to acknowledge useful discussions with Dr Grzmil, Prof. Ibrahim Adham and Dr Ashraf Mannan during Lab hours as well as with Dr Ulrich Zechner concerning DNA methylation.

Part of this work owes a lot to people of the Array Core Facility of the University of Goettingen. In particular, I am grateful to Dr. G. Salinas and Mr. L. Opitz for their invaluable help in designing, performing and interpreting miRNA microarray results. Our collaboration during the past year was crucial for this study.

This work owes a lot and it is dedicated to all my teachers, and especially to: i) Mrs L.Sergianidou, who was my first teacher in school; ii) Mr. Papasymeon, who was my first teacher in Biology; iii) Prof. N.Anagnou, my Professor in Biology in the University of Athens and my supervisor during my traineeship in the Laboratory of Molecular Biology in the University of Athens; iv) Dr. S.Kamakari, who was my lab supervisor in the previous mentioned lab; v) Dr. G.Nasioulas, Director of the Molecular Biology Department of Hygeia Hospital in Athens and my supervisor there; vi) Prof B.Zoll who teaches me Medical Genetics in the Institute of Human Genetics of the University of Goettingen and finally to Prof. W.Engel who teaches me Medical Genetics and Stem Cell Biology in the same Institute.

Finally, this work would not have existed without the help of my parents and my wife. I thank them a lot for their patience and support. 


\section{Curriculum Vitae}

My name is Athanasios Zovoilis, son of Ioannis Zovoilis and Konstantina Zovoili and I was born in Amarousio Attikis, Greece, on $19^{\text {th }}$ of May, 1982. In 2000 I graduated from the $1^{\text {st }}$ High School of Chalkida, Greece, with the note 19.7/20. From October 2000 until August 2006 I studied medicine in the Medical Faculty of the University of Athens, Greece, from where I have graduated with the note "very good". From November 2002 until November 2004 I was on my own initiative trainee in the Department of Molecular Biology of Hygeia Hospital in Athens, where I have participated in research projects concerning diagnosis of ret gene mutations for MEN 2 syndrome in patients with medullary thyroid carcinoma and their families. From December 2004 until July 2005 I have been trainee in the Molecular Biology Laboratory of the Medical Faculty of the University of Athens, where I assisted in experimental and literature research concerning expression profile of a novel gene on chromosome 5q23. From December 2005 until June 2006 I was PJ student in the University Hospital of Cologne, Germany. Since September 2006 I have been doctoral student in the Institute of Human Genetics of the University of Goettingen, where I also do my specialization in Medical Genetics. I belong to the research group of Prof. W. Engel and I have been working on pluripotent stem cells and microRNAs, which are together with bioinformatics my main research interests. 